\title{
Untersuchungen zur Polymerdynamik an laserdeponierten Poly(alkyl methacrylat)-Filmen
}

\author{
Dissertation \\ zur Erlangung des Doktorgrades \\ der Mathematisch-Naturwissenschaftlichen Fakultäten \\ der Georg-August-Universität zu Göttingen
}

vorgelegt von

Andreas Meschede

aus Warstein

Göttingen 2010 
Referent:

Korreferent:

Tag der mündlichen Prüfung:
Prof. Dr. H.-U. Krebs

Prof. Dr. K. Samwer

27. Januar 2010 


\section{Inhaltsverzeichnis}

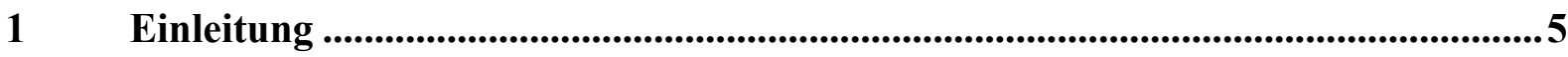

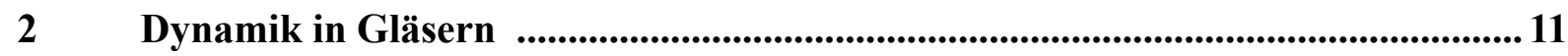

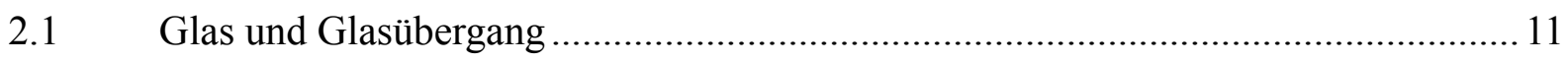

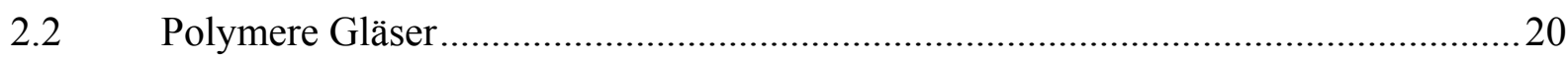

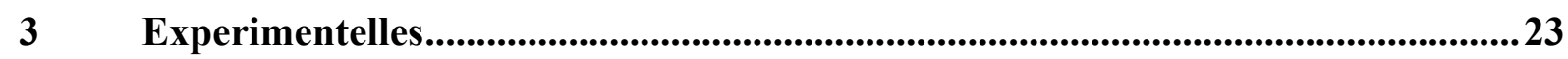

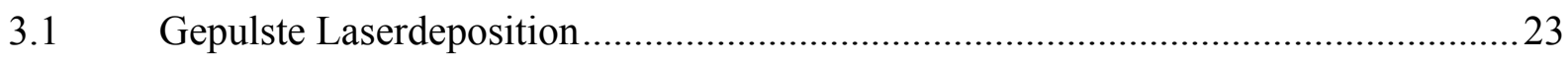

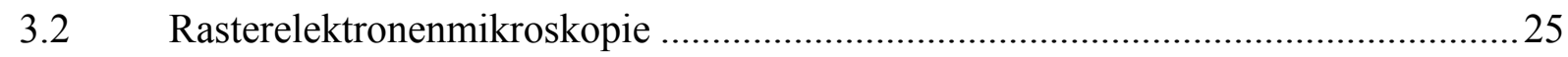

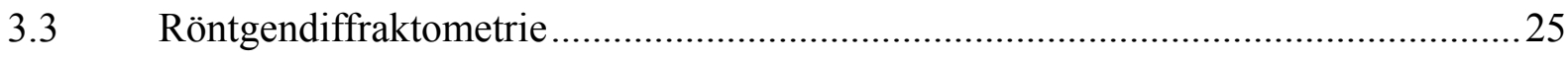

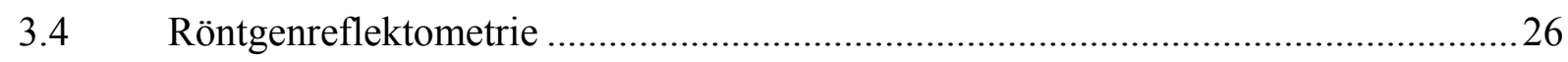

3.5 Fourier Transformations Infrarot-Spektroskopie ............................................26

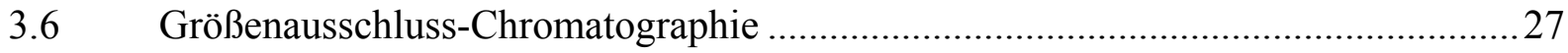

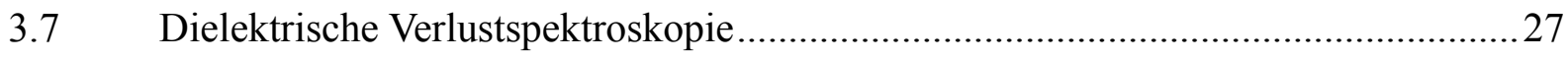

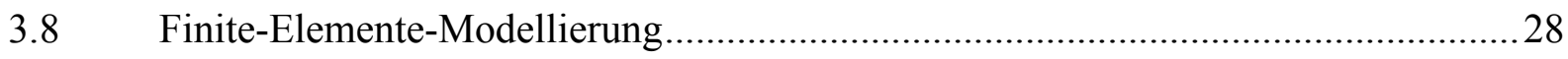

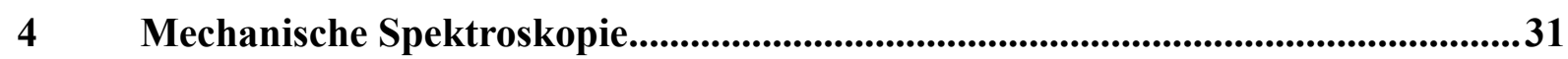

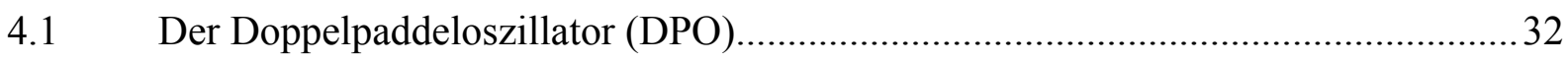

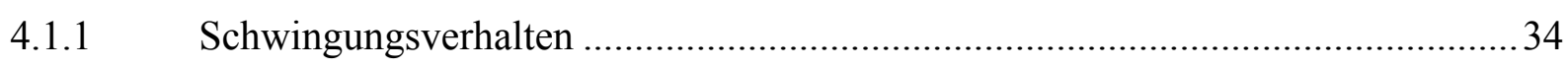

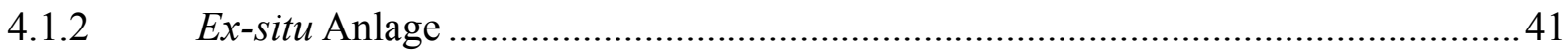

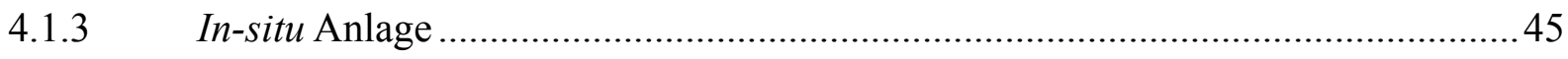

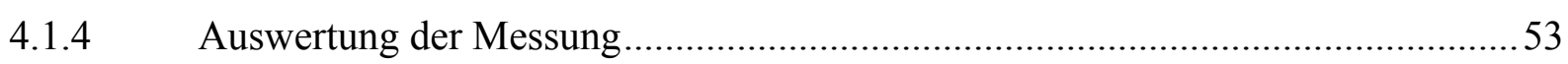




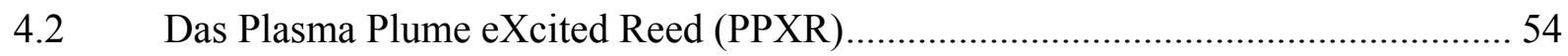

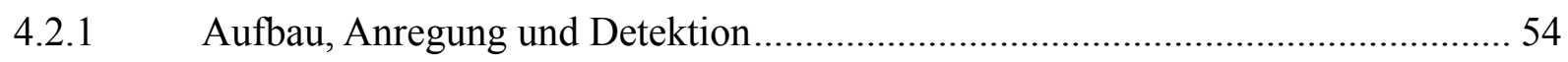

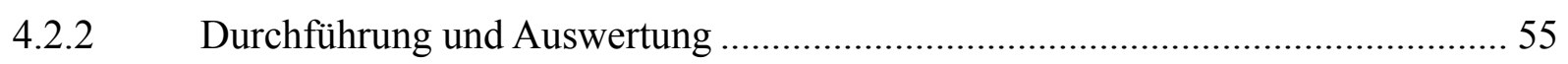

$5 \quad$ Laserdeposition: Prozess und Eigenschaften ................................................... 59

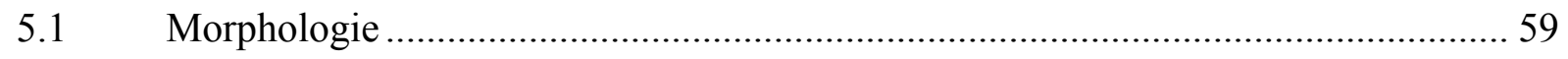

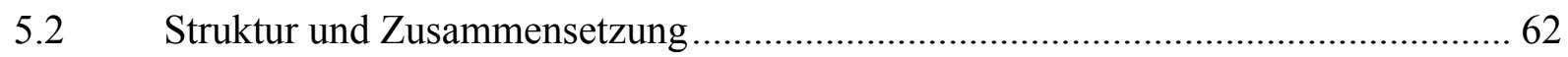

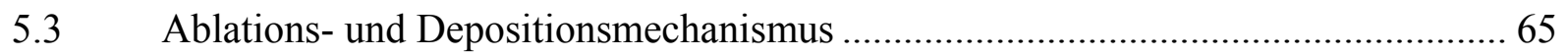

$6 \quad$ Relaxation in Poly(ethyl methacrylat).......................................................................... 69

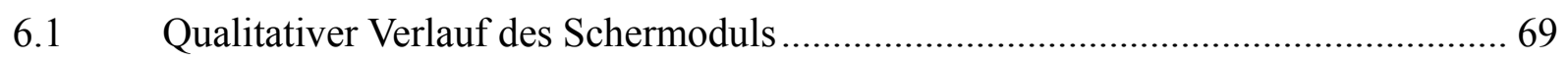

6.2 Quantitative Bestimmung des Elastizitätsmoduls .............................................. 75

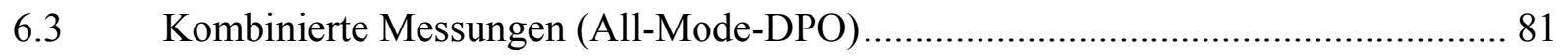

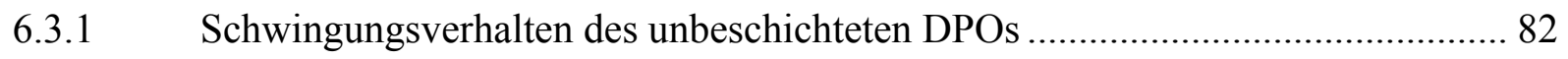

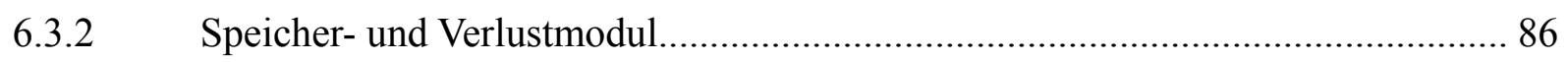

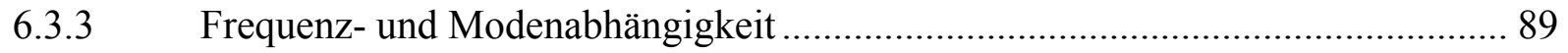

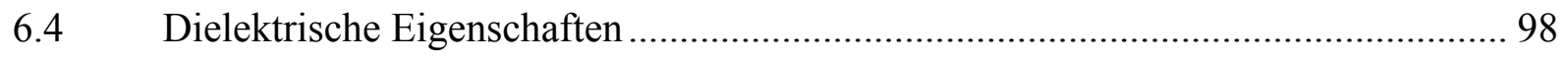

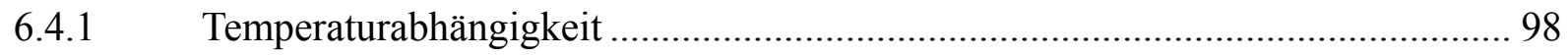

6.4.2 Abhängigkeit von der Temperatur-Vorgeschichte ............................................ 99

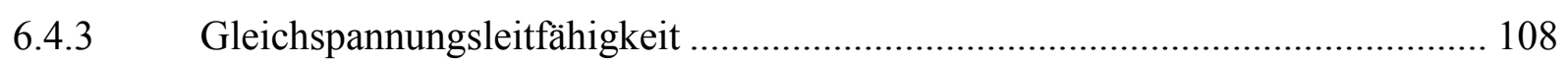

6.5 Strukturelle Änderungen bei Auslagerung .......................................................... 110

6.6 Zusammenfassende Diskussion der Relaxationseigenschaften..............................111

6.6.1 Thermisch induzierte Relaxation ................................................................. 112

Verhalten relaxierter Proben ....................................................................... 116

$7 \quad$ Vergleich mit Poly(methyl methacrylat) und Poly(butyl methacrylat)................ 120

7.1 Mechanische Dämpfung in Poly(methyl methacrylat)...................................... 120

7.2 Mechanische Dämpfung in Poly(butyl methacrylat)......................................... 129

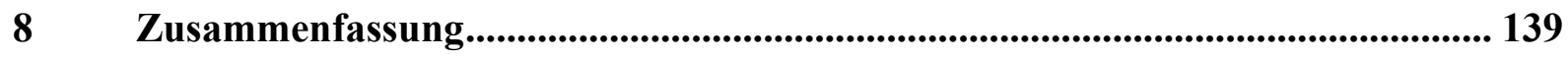

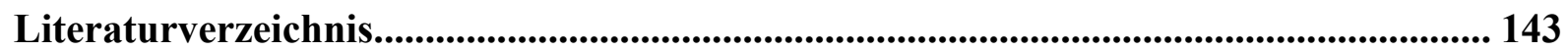




\section{Einleitung}

Der beispiellose Einfluss von Polymeren auf unser tägliches Leben wird sofort deutlich bei der Vorstellung, dass das Erbgut jedes biologischen Systems aus polymerem Material aufgebaut ist [1]. Seitdem der Mensch im 20. Jahrhundert gelernt hat, künstliche Polymere zu synthetisieren, steigt auch der Einfluss organischer Werkstoffe drastisch an, s. d. ihr Produktionsvolumen längst das von klassischen metallischen Werkstoffen wie beispielsweise Stahl überschritten hat [2]. Polymere Werkstoffe können inzwischen mit maßgeschneiderten Eigenschaften hergestellt werden, die im Wesentlichen durch die Struktur der sich wiederholenden Monomereinheiten mit durchgehender Hauptkette und abzweigenden Nebengruppen, durch ihr Molekulargewicht und durch die Vernetzung benachbarter Makromoleküle bestimmt werden. Sie zeichnen sich durch mannigfaltige Vorteile aus. Einfache Beispiele dafür sind etwa eine geringe Dichte, einfache Formbarkeit, hohe Beständigkeit gegen Korrosion, gute Verfügbarkeit ${ }^{1}$ und insbesondere auch geringe Kosten [3].

Von besonderem Interesse, insbesondere aufgrund der fortschreitenden Miniaturisierung im Bereich der Mikroelektronik, aber auch für optische, katalytische, mikrosensorische und pharmazeutische Anwendungen sind dünne Filme aus organischen Materialien [4]. Mit der Entwicklung leitfähiger, bzw. halbleitender Polymere, die Heeger, MacDiarmid und Shirakawa im Jahre 2000 den Nobelpreis für Chemie einbrachte, ist zusätzlich eine ganze Schar neuer möglicher Anwendungen etwa in der Elektrophotographie oder im Bereich von Leuchtdioden, Bildschirmen und Solarzellen erschlossen worden [5-7]. Aber auch vergleichsweise einfache mechanische oder korrosionshemmende Oberflächenvergütungen, wie z.B. Lacke gehören zu den typischen Anwendungen.

\footnotetext{
${ }^{1}$ Der Umstieg der Energieversorgung von Kohle auf Öl in der Mitte der 50er Jahre war maßgeblich am Erfolg der künstlichen Polymere beteiligt, da während der Herstellung von Heizöl leichte Benzinfraktionen als „Abfallprodukt" anfallen, aus denen Ethylen und somit eine Vielzahl der darauf basierenden Kunststoffe kostengünstig hergestellt werden können.
} 
Als Herstellungsverfahren werden meist nasschemische Prozesse verwendet, bei denen das Polymer in Lösung auf entsprechende Substrate aufgetragen wird, s. d. das Lösungsmittel verdampft und einen Film auf dem Substrat zurücklässt. Bei der Rotationsbeschichtung (engl. spin-coating), der industriell am weitesten verbreiteten Methode, wird das gelöste Polymer auf ein rotierendes Substrat getropft, um einen möglichst homogenen Film auf der gesamten Substratoberfläche zu erreichen. Bei geeigneter Wahl der vielfältigen Depositionsparameter, die u.a. sowohl von der Rotation als auch von Art und Konzentration der Lösung abhängen, können die chemischen und physikalischen Eigenschaften der Filme gut und reproduzierbar eingestellt werden [8].

Ist der nasschemische Prozess unerwünscht, etwa bei der Herstellung von komplexeren Strukturen wie Multischichten oder Kompositsystemen, sind lösungsmittelfreie Prozesse notwendig. Hier ist das Verfahren der Plasmapolymerisation etabliert, bei der die Schicht durch chemische Reaktionen von Monomeren innerhalb eines Niederdruckplasmas auf dem Substrat entsteht [9]. Die Anwendbarkeit dieser Methode ist aber auf wenige Polymere begrenzt und die produzierten Schichten weisen depositionsbedingt einen hohen Vernetzungsgrad auf.

Ende der achtziger Jahre wurde durch Hansen und Robitaille die bis dahin zur Herstellung qualitativ hochwertiger Filme unterschiedlichster Materialklassen etablierte und äußerst flexible Depositionsmethode der gepulsten Laserdeposition zur Produktion von polymeren Dünnschichten eingesetzt [10]. Dabei wurden u.a. auch Filme aus Poly(methyl methacrylat), PMMA, hergestellt. PMMA (technisch auch PLEXIGLAS ${ }^{\circledR}$ ), das seit 1933 synthetisch hergestellt werden kann, ist ein in der technischen Anwendung weit verbreiteter, transparenter Thermoplast, der überwiegend als bruchfester Glasersatz verwendet wird. Als dünner Film wird PMMA z.B. für medizinische Anwendungen oder als Schutzschicht gegen Elektronenstrahlung eingesetzt. Technologisch besonders relevant ist die Verwendung von PMMA als Photoresist für die Lithographie [11]. Die Anforderung kleiner lithographischer Strukturen und somit der Einsatz kürzerer Wellenlängen bis in den UV-Bereich initiierte somit detaillierte Untersuchungen der Wechselwirkung von PMMA mit kurzwelliger Strahlung im besonderen Hinblick auf den Ablationsprozess [12-14]. Aufbauend auf der daraus entstandenen Fülle wissenschaftlicher Erkenntnisse erreichte PMMA somit den Status eines idealen Modellsystems für die Laserdeposition von Polymeren. Dennoch führt die Abhängigkeit von den experimentellen Bedingungen wie z.B. verwendeter Laserwellenlänge, Energiedichte der Strahlung, Umgebungsdruck oder Reinheit des Targetmaterials dazu, dass über einen großen Parameterraum noch immer kein geschlossenes Modell erstellt werden konnte. 
Durch intensive Untersuchungen von Süske und Scharf $[15,16]$ konnte in den letzten Jahren zumindest für den Wellenlängenbereich des KrF-Excimer-Lasers bei $248 \mathrm{~nm}$ und typischen Laserfluenzen von einigen $\mathrm{J} / \mathrm{cm}^{2}$ eine umfassende Beschreibung des Ablations- und Depositionsprozesses gewonnen werden. Die hergestellten PMMA-Filme besitzen einige im Vergleich zum Volumenmaterial oder nasschemisch hergestellten Dünnschichten andersgeartete Eigenschaften, die durch Variation der Depositionsparameter im gewissen Umfang eingestellt werden können [17, 18]. Von besonderem Interesse ist dabei die Möglichkeit, die mechanischen Eigenschaften durch thermische Behandlung zu verändern.

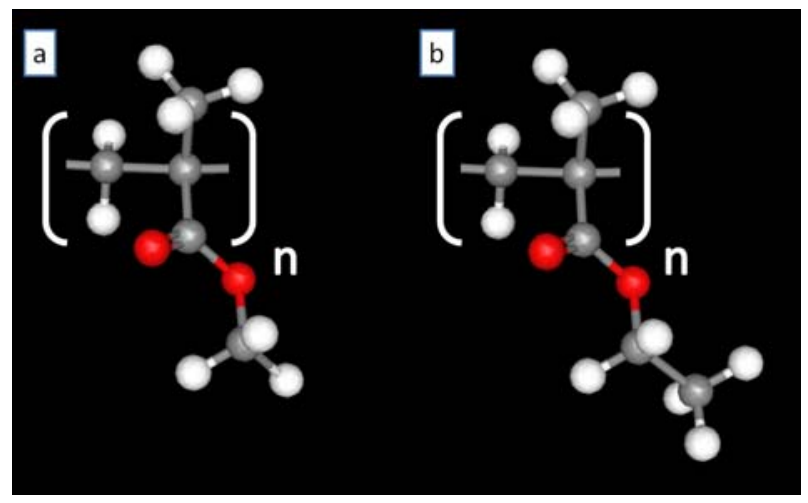

Abbildung 1.1: Strukturformeln von a) Poly(methyl methacrylat) und b) Poly(ethyl methacrylat).

Ausgehend von den Vorarbeiten zur Laserdeposition von PMMA folgt im Rahmen dieser Arbeit in logischer Konsequenz die Erweiterung der Untersuchungen auf weitere Vertreter der Poly(alkyl methacrylat)e (PAMA). Als nächsthöherer Homologe ist in Abbildung 1.1 neben der Strukturformel von PMMA die von Poly(ethyl methacrylat) (PEMA) dargestellt. Die chemische Struktur in der Hauptkette bleibt in der Reihe der PAMAs erhalten, während der Alkylester in der Seitengruppe durch den sukzessiven Einbau weiterer $\mathrm{CH}_{2}$-Einheiten verlängert wird. Beim Poly(butyl methacrylat) (PBMA), das ebenfalls in dieser Arbeit untersucht wird, befinden sich insgesamt drei $\mathrm{CH}_{2}$-Einheiten in der Seitengruppe. In einem einfachen Modell zur Erklärung der mechanischen Eigenschaften führt die Verlängerung der Seitengruppe zu einem größeren Abstand zwischen den verschiedenen Makromolekülen und dient somit als Weichmacher (interne Plastifikation): Durch das eingebrachte zusätzliche freie Volumen wird die Bewegung der Segmente der Hauptkette vereinfacht und die Glasübergangstemperatur wird herabgesetzt. Aufgrund der verringerten Glasübergangstemperatur fällt bereits das PEMA für die PMMA-typischen Anwendungen, z. B. als abriebresistente Oberflächenversiegelung, aus. Stattdessen wird es z.B. emulgiert als Zusatz in Klebstoffen, Anstrichen oder Imprägnierungen eingesetzt. Aus akademischer Sicht sind die Methacrylate interes- 
sant, weil durch die gezielte Beeinflussung der Seitengruppe drastische Veränderungen an der Dynamik des Polymers vorgenommen werden können, während die sonstigen chemischen Eigenschaften nur schwach verändert werden.

Im Rahmen dieser Arbeit soll ausgehend vom Verständnis der Laserdeposition des PMMAs ein erweitertes Bild sowohl der Depositions- und Wachstumsmechanismen als auch der Eigenschaften der weiteren Homologen der Poly(alkyl methacrylat)e erstellt werden. Von zentraler Bedeutung ist dabei die Frage, ob Universalität in der Reihe der PAMAs gefunden werden kann. Die Frage nach der Universalität stellt sich dabei besonders in Hinblick auf die Dynamik der polymeren Systeme, die detailliert mit spektroskopischen Methoden untersucht wird. Hier soll geklärt werden, in welcher Weise die dem Fließprozess zugrundeliegenden Mechanismen durch die Unterschiede in den Seitengruppen beeinflusst werden. Dabei wird sich herausstellen, dass die Eigenschaften laserdeponierter PAMA-Filme in weiten Bereichen mit Volumenmaterial vergleichbar sind und beispielsweise die typischen Eigenschaften polymerer Gläser aufweisen. Es werden aber auch spezifische Eigenschaften gefunden, die direkt auf Prozesse während der Laserdeposition zurückzuführen sind. Hier zeigt sich insbesondere die durch Relaxationsprozesse bestimmte starke Veränderung der Filmeigenschaften bei Auslagerung. Diese spezifischen Eigenschaften werden im Detail beschrieben und es wird versucht, ein umfassendes Verständnis der zugrundeliegenden Mechanismen aufzubauen.

Im Detail gliedert sich die vorliegende Arbeit wie folgt:

In Kapitel 2 wird zunächst durch die Einführung etablierter Konzepte der Glasphysik der theoretische Hintergrund zur Beschreibung amorpher Systeme bereitgestellt. Insbesondere wird dabei auf die Besonderheiten der Dynamik in Polymeren eingegangen.

Kapitel 3 enthält neben einer detaillierten Beschreibung der verwendeten Depositionsaufbauten einen Überblick über die experimentellen Methoden, die zur umfassenden Charakterisierung der untersuchten Schichtsysteme angewendet wurden.

Der zentralen Untersuchungsmethode dieser Arbeit, der mechanischen Spektroskopie, ist mit Kapitel 4 ein eigener Abschnitt gewidmet. Hier werden detailliert die verwendeten experimentellen Anlagen, der Doppelpaddeloszillator und das Plasma Plume eXcited Reed, sowie die zur quantitativen Auswertung der Messungen notwendige klassische Mechanik vorgestellt. Ein weiterer Fokus liegt auf der Beschreibung der konstruktiven Veränderungen und der Optimierung der unterschiedlichen Messaufbauten, sowie der Darstellung der dabei ver- 
wendeten Rechnungen mit Finite-Elemente-Modellierung. Der umfassenden Beschreibung der im Rahmen dieser Arbeit entwickelten in-situ-Apparatur wird dabei besonderer Platz eingeräumt.

Kapitel 5 beschäftigt sich mit der Frage, in wie weit die bekannten Mechanismen der Laserdeposition des PMMAs auf weitere Homologen der PAMAs verallgemeinert werden können. Dazu werden der Depositionsprozess des Poly(ethyl methacrylat)s und die Eigenschaften der resultierenden Filme detailliert analysiert und direkt mit den vom PMMA bekannten Ergebnissen verglichen.

In Kapitel 6 wird das mechanische Verhalten laserdeponierter PEMA-Filme quantitativ analysiert und die zugrundenliegende Dynamik untersucht. Im Besonderen werden dabei die bei Temperaturbehandlung auftretenden Relaxationsprozesse untersucht. Über die Analyse der dielektrischen Eigenschaften und strukturellen Änderungen der Schichten wird versucht, die für diese Relaxationen verantwortlichen Mechanismen zu identifizieren.

Zur Untersuchung des Einflusses der unterschiedlichen Seitengruppen ist die quantitative Analyse der mechanischen Eigenschaften nicht zwingend notwendig. Stattdessen ist bereits die mechanische Dämpfung ein hervorragender Indikator für Relaxationsprozesse. In Kapitel 7 werden Dämpfungsmessungen an PMMA und PBMA-Schichten demonstriert und die Unterschiede zwischen den drei Homologen diskutiert. Der Vergleich der mit unterschiedlichen Methoden bestimmten Ergebnisse zur mechanischen Dämpfung aller untersuchter PAMASchichten wird weiterhin Rückschlüsse auf die Abhängigkeit der Relaxationseigenschaften von Art der Schwingung zulassen.

In Kapitel 8 werden die gewonnenen Ergebnisse dieser Arbeit schließlich kurz zusammengefasst. 


\section{Dynamik in Gläsern}

Gläser werden zwar bereits lange erforscht und in der Technologie schon seit mehr als drei Jahrtausenden angewandt (In Ägypten wurden bereits ca. 1400 v. Chr. Gefäße aus Glas hergestellt), dennoch bilden die physikalischen Konzepte jenseits der Phänomenologie eines der in der aktuellen Forschung am stärksten diskutierten Themen. Anderson verdeutlichte dies 1995 in einer Umfrage des Science Magazins: „The deepest and most interesting unsolved problem in solid state theory is probably the nature of glass and the glass transition" [19]. Ein Hauptgrund für die Problematik der theoretischen Beschreibung eines Glases liegt in der Ermangelung der Fernordnung, die eine einfache Modellierung über periodische Strukturen, wie bei Kristallen üblich, verhindert.

Nach einem kurzen Überblick über die Phänomenologie des Glasübergangs wird im Folgenden zunächst der Glasübergang im einfachen Modell harter Kugeln ohne innere Freiheitsgrade diskutiert. Daraufhin werden einige für diese Arbeit relevantere Konzepte der Dynamik in Polymeren vorgestellt. Aufgrund ihrer bedeutend höheren strukturellen Komplexität zeigt dabei auch die Dynamik ein komplexeres Verhalten, das auf zusätzliche intrinsische Relaxationsmechanismen zurückzuführen ist.

\subsection{Glas und Glasübergang}

Nach Elliot ist ein Glas „ein amorpher Festkörper, der einen Glasübergang zeigt“ [20]. Bereits bei dieser sehr allgemeinen Definition wird deutlich, wie schwer die genaue Beschreibung dieser Materialklasse fällt. Dennoch sind hier bereits zwei wichtige Punkte genannt.

Zunächst ist ein Glas amorph (gr.: „ohne Gestalt“), d.h. die Atompositionen im Glas unterliegen keiner Fernordnung. Statt fester Gitterparameter, wie im Kristall, werden bei Gläsern Korrelationsfunktionen verwendet, um ihre Nahordnung zu beschreiben. Die Einfachste ist die Paarkorrelationsfunktion $\rho(r)$ : Sie beschreibt die über die Zeit und alle Teilchen gemittelte 
Wahrscheinlichkeit, ein Teilchen im Abstand $r$ von einem weiteren Teilchen zu finden. Die in Abbildung 2.1 skizzierte Paarkorrelationsfunktion eines schematischen Glases zeigt breite Maxima, deren Ursache in der Bildung von Koordinationsschalen liegt [20, 21].

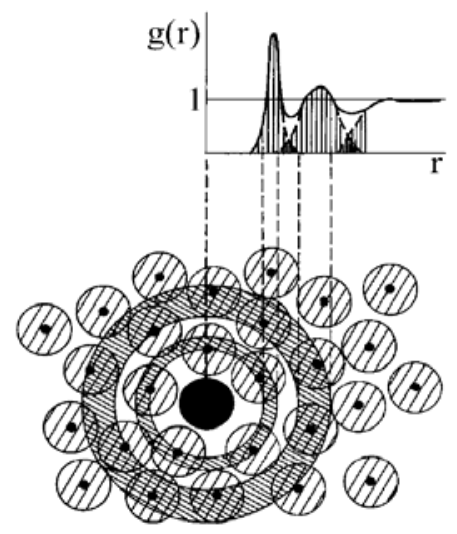

Abbildung 2.1: Die Paarkorrelationsfunktion eines amorphen Systems zeigt breite Maxima, die auf Koordinationsschalen zurückzuführen sind [20].

Der zweite Teil der Definition beinhaltet den Glasübergang, der sich über den Prozess des Abkühlens eines Glasbildners aus der Schmelze erklären lässt. Beim schnellen Abkühlen einer Flüssigkeit ändert sich der Temperaturverlauf ihrer extensiven thermodynamischen Größen wie Volumen, Enthalpie oder Entropie am Glasübergang.

Abbildung 2.2 zeigt diesen Temperaturverlauf exemplarisch für die Entropie. Im thermodynamischen Gleichgewicht (durchgezogene schwarze Linie) durchläuft das Material beim Erreichen der Temperatur $T_{X}$ mit der Kristallisation einen Phasenübergang 1. Ordnung. Die Kristallisation lässt sich aber durch schnelles Abkühlen unterdrücken und man erreicht den NichtGleichgewichtszustand der unterkühlten Schmelze, die als metastabiler Gleichgewichtszustand angesehen werden kann. Erst bei der Glasübergangstemperatur $T_{g}$ friert die Dynamik des Systems soweit ein, dass das System dem Gleichgewichtsverhalten der Flüssigkeit nicht mehr folgen kann. Dabei ist, wie in Abbildung 2.2 angedeutet, die Glasübergangstemperatur keine universelle Materialkonstante. Vielmehr erfolgt der Glasübergang bei raschem Abkühlen bereits bei hohen Temperaturen (blaue Kurve); beim langsamen Abkühlen werden bei einer tieferen Temperatur Zustände mit geringerer Entropie eingefroren (rote Kurve). Die jeweilige kühlratenabhängige Glasübergangstemperatur wird als die dem eingeschreckten Zustand zugehörige fiktive Temperatur bezeichnet [22]. Bei unendlich langsamer Abkühlung (gestrichelte Linie) schneiden sich die Entropieverläufe von Glas und Kristall bei der KauzmannTemperatur $T_{K}$. Diese Temperatur kann als die fiktive Temperatur eines „idealen“ Glases ver- 
standen werden und wird im Zuge des Kauzmann-Paradoxons, also der Unmöglichkeit der Gleichheit der Entropie von ungeordnetem Glas und geordnetem Kristall, vielfältig diskutiert [23]. Tatsächlich scheint $T_{K}$ aber für die Physik des Glasübergangs keine relevante Bedeutung zu haben [24]. Stattdessen gilt die über der Glasübergangstemperatur liegende kritische Temperatur $T_{C}$ der Modenkopplungstheorie [7,8] als die Temperatur, bei der sich die Dynamik des Glases grundlegend verändert (s. Seite 19).

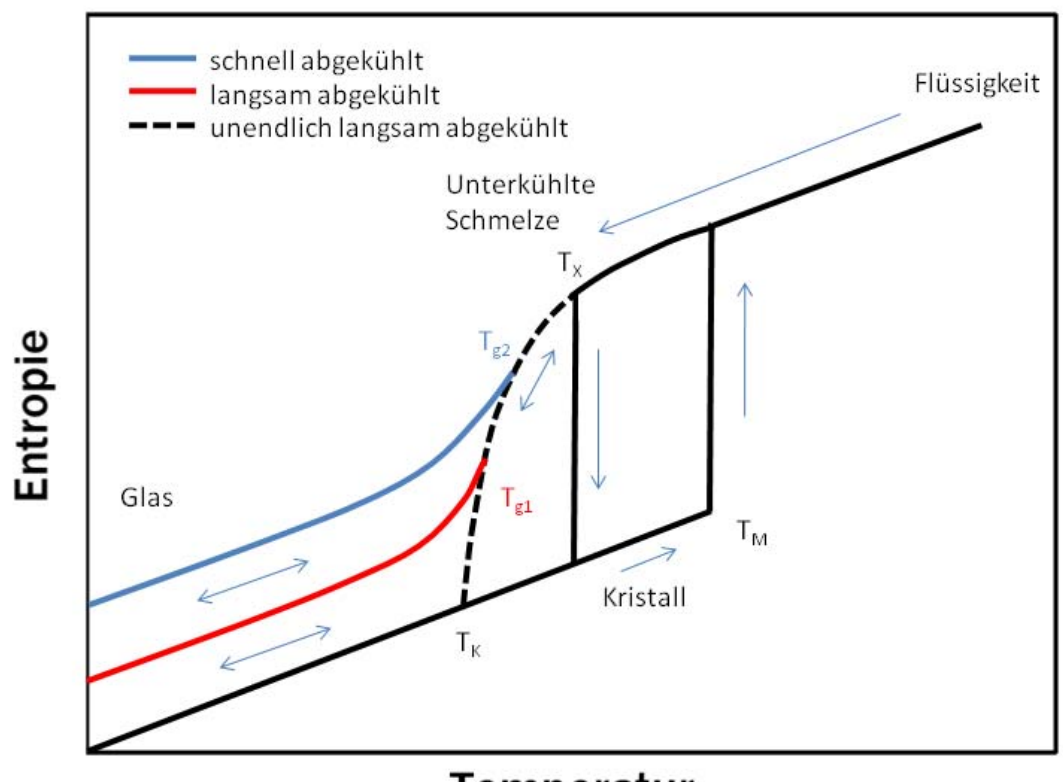

Temperatur

Abbildung 2.2: Schematischer Verlauf der Entropie für Glasbildner und Kristall beim Abkühlen aus der Schmelze. Je nach Wahl der Abkühlrate können unterschiedliche Zustände im Glas erreicht werden (nach [22]).

In der Zeitabhängigkeit eines glasbildenden Systems gibt es nun also drei Temperaturbereiche, von denen zwei als stationär anzusehen sind. Für Temperaturen weit unterhalb der Kauzmann-Temperatur verbleibt das Glas in seinem eingefrorenen Zustand mit der zugehörigen fiktiven Temperatur ${ }^{1}$. Auch für Temperaturen oberhalb von $T_{C}$, also in der unterkühlten Schmelze, verharrt das System im metastabilen Gleichgewicht, solange keine Kristallisation auftritt. Im Temperaturintervall dazwischen ist die Temperaturabhängigkeit bei gleichbleibender Heiz-/Kühlrate zunächst reversibel, wie in Abbildung 2.3 erneut für die Entropie des Sys-

\footnotetext{
${ }^{1}$ Für sehr lange Zeiten gibt es sicherlich eine gewisse Wahrscheinlichkeit, dass sich ein eingefrorenes System verändert, allerdings sind diese für die hier betrachteten Zeitskalen nicht relevant. Der häufig verwendete Mythos des ,fließenden“ Kirchenfensters, das im Laufe der Jahrhunderte aufgrund der Schwerkraft am unteren Rand dicker wird als am oberen Rand, ist falsch. Die unterschiedlichen Dicken der Scheiben beruhen auf Unregelmäßigkeiten bei der Herstellung [25] R. C. Plumb, Antique Windowpanes and the Flow of Supercooled Liquids, Journal of Chemical Education 66, 994 (1989).
} 
tems skizziert. Heizt man z. B. aus Zustand 2 mit der vorangegangenen Kühlrate wieder auf, so bleiben der Zustand und die fiktive Temperatur erhalten. Die Entropie knickt bei der vorherigen Temperatur ab. Bei Veränderung der Heizrate gegenüber der vorigen Kühlrate, verlässt das System den reversiblen Verlauf und die fiktive Temperatur wird zu höheren (Verlauf 1', höhere Heizrate) oder tieferen Temperaturen (Verlauf 3', kleinere Heizrate) verschoben. Mit dem Erreichen des metastabilen Gleichgewichts der unterkühlten Schmelze liegen wieder alle Kurven übereinander.

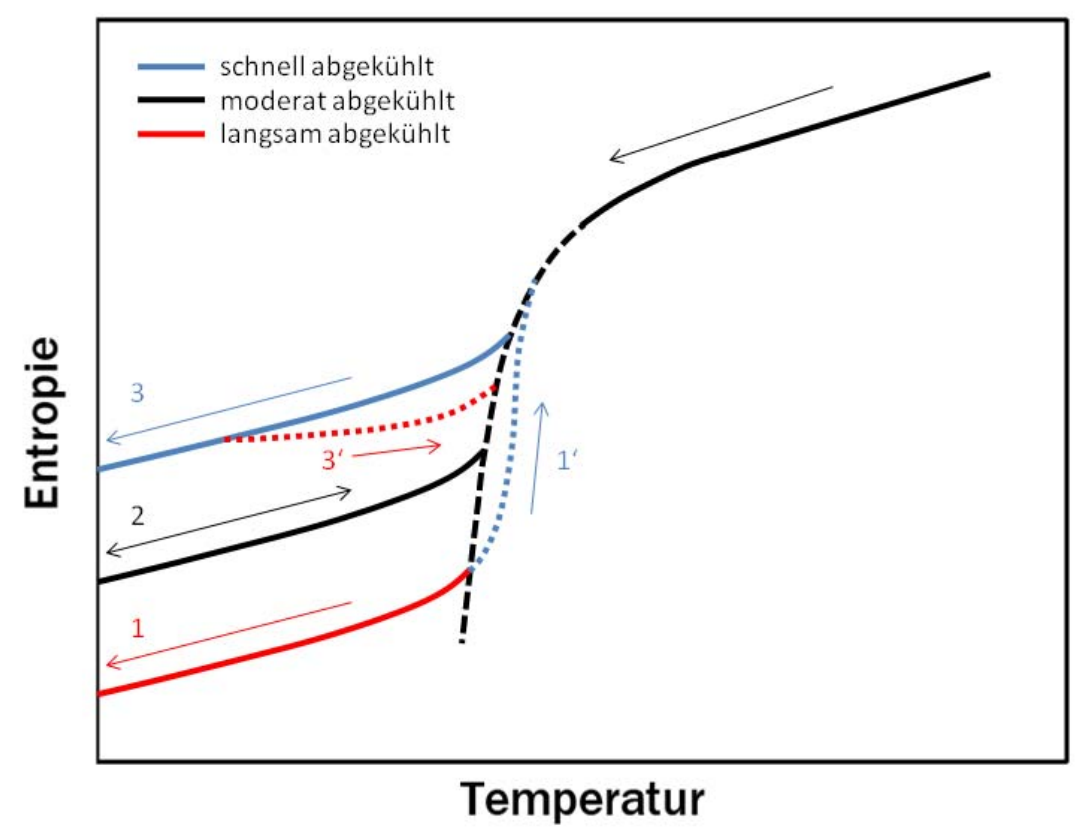

Abbildung 2.3: Schematischer Temperaturverlauf der Entropie eines Glases bei unterschiedlicher Kühl- und Heizrate (nach [22]). Linie 1 zeigt den Verlauf eines langsam, Linie 2 den eines moderat und Linie 3 den eines rasch abgekühlten Systems. Bei jeweils gleichbleibender Kühl- und Heizrate ist der Verlauf reversibel. Bei geänderter Heizrate (schnelleres Heizen bei Linie $1^{6}$, langsameres Heizen bei Linie $3^{\circ}$ ) nähern sich die Kurven dem Verlauf der Kühlkurven an, die der neuen Heizrate entsprechen.

Wird die unterkühlte Schmelze nicht erreicht, bleibt eine Vorgeschichtsabhängigkeit im System erhalten. Es entsteht ein Mischzustand aus den zu den jeweiligen Heiz- und Kühlraten gehörigen Zuständen. Altern (physical aging) bezeichnet dabei das Überführen des Glases in einen Zustand mit niedrigerer fiktiver Temperatur durch langsames Erhitzen [26]. Durch schnelles Abschrecken lässt sich ein System in einen Zustand mit höherer fiktiver Temperatur verjüngen (rejuvenation).

Eine Möglichkeit, den dynamischen Glasübergang zu definieren, bietet die Viskosität. Da das Einfrieren einer Flüssigkeit zum Glas, wie oben beschrieben, aber kontinuierlich erfolgt, ist 
die genaue Festlegung willkürlich. In der Literatur wird ein Zustand konventionsgemäß ab einer Viskosität von $\eta=10^{12}$ Pas als fest, also als Glas bezeichnet $[9,10]$. Unterschiedliche Systeme können stark unterschiedliche Temperaturabhängigkeiten der Viskosität zeigen. Dies kann zur Katalogisierung der Gläser in „starke“ und „fragile“ Glasbildner benutzt werden.

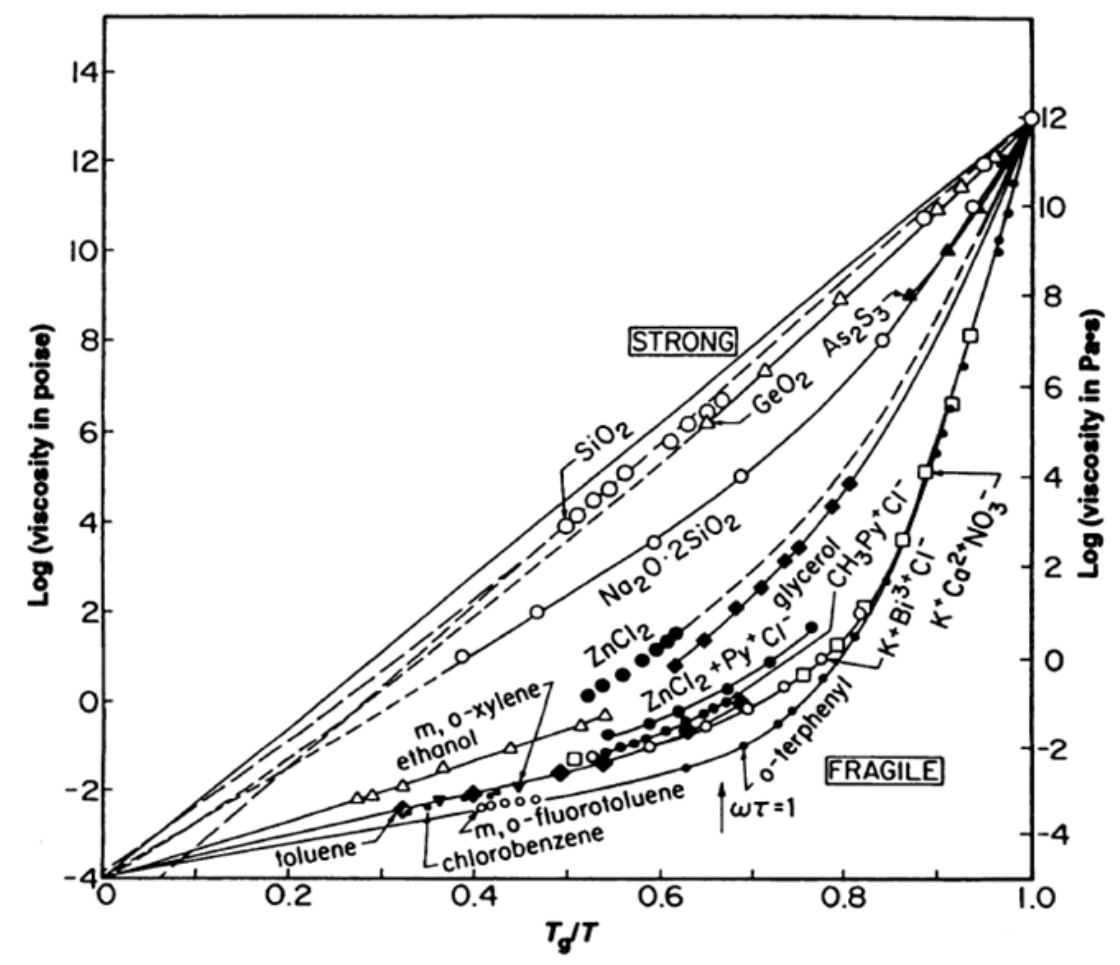

Abbildung 2.4: Angell-Plot zur Charakterisierung glasbildender Systeme nach der Temperaturabhängigkeit ihrer Viskosität [27]. Starke Glasbildner, wie die oxydischen Gläser, zeigen eine geringe Fragilität, also vergleichsweise wenig Temperaturabhängigkeit der Viskosität. Bei fragilen Systemen, wie z. B. den Polymeren, ändert sich die Viskosität bei Erreichen der Glastemperatur besonders drastisch.

Abbildung 2.4 zeigt die von Angell vorgeschlagene Auftragung der logarithmischen Viskosität verschiedenster Glasbildner gegenüber $T_{g} / T$ zur Unterscheidung der Fragilität $[9,10]$. Die starken Glasbildner wie die oxydischen Gläser zeigen nur geringe Abweichungen von Arrhenius-Verhalten, was auf einen einfach thermisch aktivierten Fließprozess schließen lässt. Bei Polymeren und anderen fragilen Glasbildnern ändert sich die Viskosität bei Annäherung an die Glasübergangstemperatur bedeutend stärker, die „Aktivierungsenergie“ nimmt mit sinkender Temperatur stark zu. Die Steigung der Viskosität am Glasübergang gilt als Maß für die Fragilität und wird als Fragilitätsindex $m$ bezeichnet. Für das fragile Glas PMMA beträgt $m=145$ [28] und nimmt für die weiteren Poly(alkyl methacrylat)e mit steigender Länge der Seitengruppe ab [29, 30]. Für die PAMAs gilt: je länger die Estergruppe, desto stärker das Glas. 
Der gekrümmte Verlauf der Viskosität der fragilen Glasbildner lässt sich empirisch mit einer Vogel-Fulcher-Tammann Gleichung (VFT) beschreiben [12-14].

$$
\eta_{V F T}=\eta_{0} \cdot \exp \left(\frac{D \cdot T_{V F T}}{T-T_{V F T}}\right)
$$

wobei der Stärkeparameter $D$ über

$$
m=m_{\min }+m_{\min }^{2} \ln \left(\frac{10}{D}\right) \approx 16+\frac{590}{D}
$$

empirisch mit dem Fragilitätsindex verknüpft ist [31]. Da diese Gleichung in der Nähe der Kauzmann-Temperatur bei $T_{V F T}$ divergiert, ist sie nur oberhalb dieser gültig [32]. Der durch die Viskosität beschriebene makroskopische Fließprozess wird in der Literatur als $\alpha$-Relaxation bezeichnet. Dieser Prozess der strukturellen Umordnung hat am Glasübergang mit $\eta=10^{12}$ Pas definitionsgemäß eine Relaxationszeit von $100 \mathrm{~s}$.

Neben diesem Hauptprozess zeigen Glasbildner, abhängig von Ihrer Komplexität, eine Reihe weiterer Relaxationsmechanismen. Abbildung 2.5 zeigt schematisch die für ein einfaches glasbildendes System auftretenden Relaxationen, wie sie über eine breitbandige Messung des dielektrischen Verlustes zugänglich sind [33]. Bei niedrigen Frequenzen ist zunächst die $\alpha$-Relaxation, der bereits diskutierte Fließprozess, dominant. Mit dem „Wing“ ist auf der hochfrequenten Seite des $\alpha$-Prozesses eine Flanke angedeutet, deren Ursache lange im Fokus der wissenschaftlichen Diskussion stand und immer noch nicht abschließend geklärt werden konnte. Dabei ist die zentrale Frage, ob dieser Wing einen Vorläufer des $\alpha$-Prozesses oder eine eigenständige, verdeckte sogenannte $\beta$-Relaxation darstellt [34-38]. In Polymeren mit dielektrisch aktiven Seitengruppen lässt sich dieser „slow $\beta$-Prozess“ eindeutig durch intramolekulare Bewegungen, beispielsweise Drehungen der Seitengruppen, die nicht zu makroskopischer Verformung der Probe führen, identifizieren [39, 40]. Da aber auch weniger komplexe Gläser $\beta$-Relaxationen zeigen [41-44], setzt sich mittlerweile die Vorstellung durch, dass es sich bei dem Wing um einen eigenständigen Relaxationsprozess handelt. Als mikroskopische Ursache gelten kollektive Prozesse in sogenannten dynamischen Heterogenitäten bzw. kooperativen Regionen [38, 45-48]. Aufbauend auf den Arbeiten von Johari und Goldstein werden ,islands of mobility“, also Regionen mit geringerer Dichte und somit schnellerer Dynamik zur Erklärung herangezogen $[41,49]$. Die strukturelle Umordnung in hinreichend dichten Systemen ist 
demnach nur durch das Zusammenspiel mehrerer Teilchen in einer solchen kooperativen Region möglich.

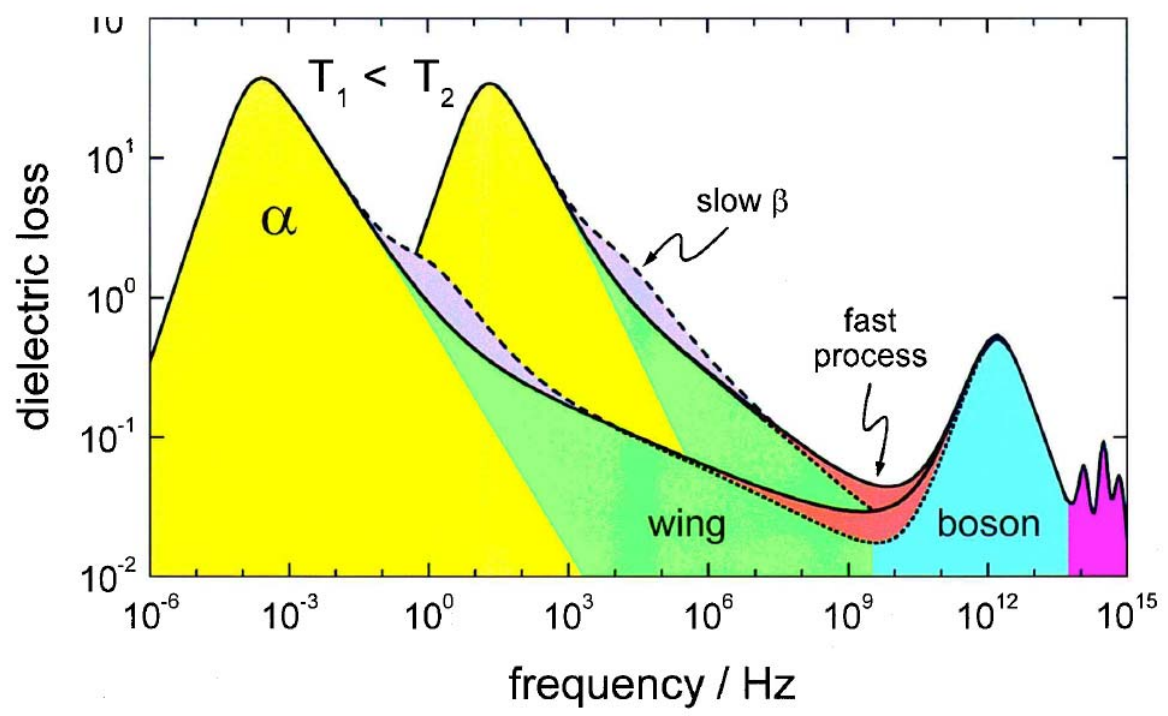

Abbildung 2.5: Der Lunkenheimer-Loidl-Plot zeigt die verschiedenen Relaxationsprozesse, die bei einem Glasbildner auftreten können, am Beispiel einer breitbandigen Messung des dielektrischen Verlustes für zwei Temperaturen [33].

$\mathrm{Zu}$ höheren Frequenzen schließt sich ein Minimum und der nahezu konstante ,nearly constant loss“ an. Die zugrundeliegende mikroskopische Bewegung in diesem Frequenzbereich ist das „cage rattling“, also das Rattern von im Käfig aus Nachbaratomen eingeschlossenen Teilchen (auch als ,fast process“" oder schneller $\beta$-Prozess beschrieben [50]). Noch etwas schneller, mit Frequenzen bis etwa $10^{10} \mathrm{~Hz}$, sind kooperative „Gitter“-Schwingungen, die bei einigen $\mathrm{THz}$ zum „Boson-Peak“ führen [51-53]. Für sehr große Frequenzen über $10^{13} \mathrm{~Hz}$ bestimmen molekulare Dreh- und Schwingungsrelaxationen mit scharfen Frequenzen im Infraroten die Dynamik.

Aus den Frequenzlagen der Maxima $f$ lässt sich über $2 \pi f \cdot \tau=1$ auf die typischen Relaxationszeiten $\tau$ schließen. Diese sind aufgrund der breiten Maxima in Abbildung 2.5 nicht diskret, sondern man beobachtet eine Verteilung verschiedener Relaxationszeiten [54]. Die Verteilung der Relaxationszeiten in der Zeitdomäne wird im Allgemeinen durch die Verwendung einer gestreckten Exponentialfunktion beschrieben. Eine solche Relaxationsfunktion ist die Antwortfunktion auf eine stufenförmige Anregungsfunktion gemäß Kohlrausch, Williams und Watts (KWW):

$$
\varphi_{K W W}=\varphi_{0} \exp \left(\left(-\frac{t}{\tau_{K W W}}\right)^{\beta_{K W W}}\right)
$$


mit der KWW-Relaxationszeit $\tau_{\mathrm{KWw}}$ und dem KWW-Exponenten $\beta_{\mathrm{KWW}}$, der Werte zwischen 0 und 1 annehmen kann und für die Streckung der Exponentialfunktion verantwortlich ist [55, 56]. Diese Gleichung ist die exakte Lösung des Integrals über eine Dichte von einfachen Debyeschen Anregungsfrequenzen, also der Superposition von unterschiedlichen Antwortfunktionen mit jeweils nur einer festen Relaxationszeit.

Der für periodische Messungen notwendige Übergang in den Frequenzraum erfolgt durch Fouriertransformation, ist jedoch nicht trivial und im Allgemeinen nur numerisch, insbesondere aber nicht eindeutig lösbar [57]. So stellt auch die häufig verwendete komplexe HavriliakNegami (HN) Funktion [58]

$$
\chi^{*}(\omega)=\chi_{\infty}+\frac{\chi_{S}+\chi_{\infty}}{\left(1+\left(i \omega \tau_{H N}\right)^{\alpha}\right)^{\gamma}}
$$

mit den asymptotischen Werten für den statischen Fall $\chi_{\mathrm{S}}$ und dem bei unendlicher Frequenz $\chi_{\infty}$, der Kreisfrequenz $\omega=2 \pi f$, der Relaxationszeit $\tau_{\mathrm{HN}}$ sowie den Parametern für Verbreiterung $\alpha$ und Asymmetrie $\gamma$ (jeweils mit Werten zwischen 0 und 1), keine exakte FourierTransformation der KWW-Funktion dar. Bei geeigneter Wahl der Parameter sind sich beide Funktionen aber sehr ähnlich und es gilt meist $\alpha \gamma=\beta_{K W W}^{1,23}[57,59]$.

In Abbildung 2.5 ist an den beiden Messungen für unterschiedliche Temperaturen erkennbar, dass die Relaxationsprozesse mit steigender Temperatur unterschiedlich stark zu höheren Frequenzen verschoben werden. Abbildung 2.6 zeigt die typischen mittleren Relaxationszeiten des $\alpha$ - und des $\beta$-Prozesses in Abhängigkeit von der reziproken Temperatur. Während die Relaxationszeiten des $\alpha$-Prozesses einen Vogel-Fulcher-Tammann-Verlauf zeigen (Formel 2.1 mit $\eta \sim \tau)$, verhalten sich $\beta$-Relaxationen Arrhenius-typisch [41, 60], s. d. es nach diesem Bild zwangsläufig einen Bereich geben muss, an dem sich die Verläufe treffen. Die Überlagerung der beiden Prozesse wird „merging“ genannt; die merging-Zone kann im Experiment durch Variation von Messfrequenz und Temperatur untersucht werden. 


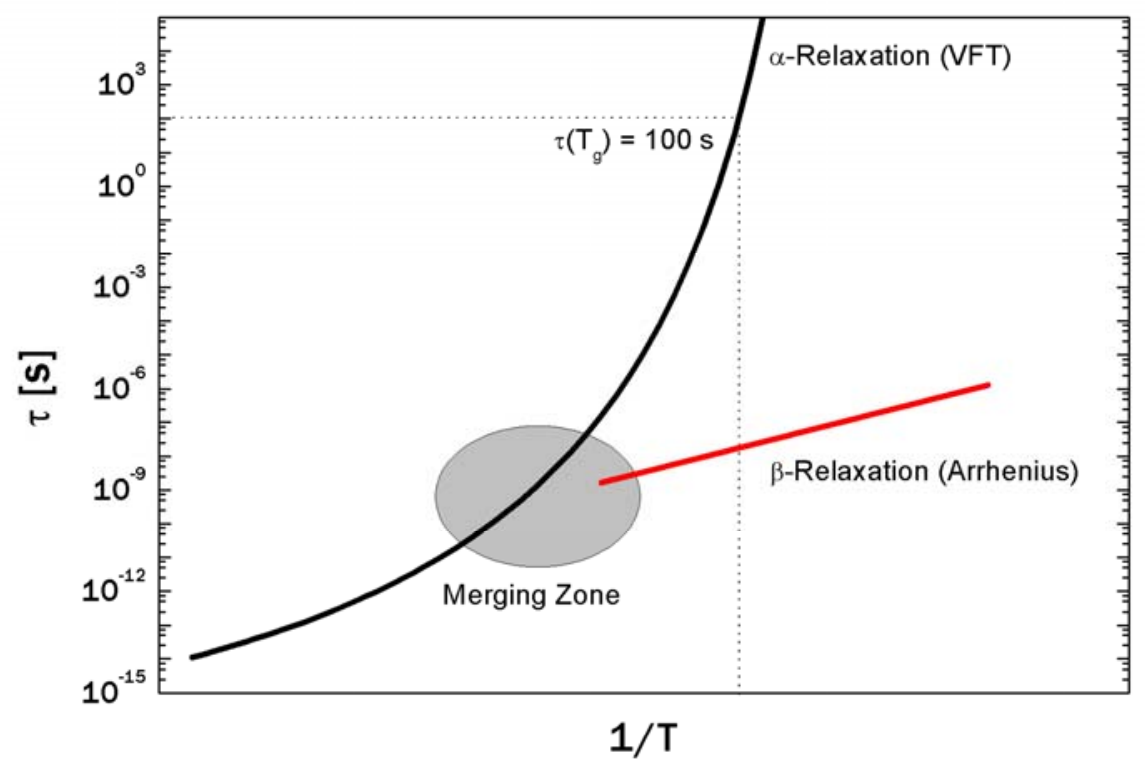

Abbildung 2.6: Schematische Abhängigkeit von charakteristischen Relaxationszeiten von $\alpha$ - und $\beta$-Prozess. Während der $\alpha$-Prozess ein VFT-Verhalten zeigt, folgt die $\beta$-Relaxation einem Arrhenius-Verlauf. Im merging-Gebiet nähern sich die Relaxationszeiten beider Prozesse an.

Zur theoretischen Modellierung des Glasübergangs wird meist von Vielteilchenmodellen mit einer starken intermolekularen Wechselwirkung ausgegangen. Alle oben beschriebenen Relaxationsmoden können statistisch über Potentialmodelle beschrieben werden, in dem ein schwingendes Teilchen (oder eine Schar von Teilchen) auf eine Potentialbarriere trifft und diese abhängig von der Temperatur mit einer gewissen Wahrscheinlichkeit überschreiten kann. Obwohl es bisher keine umfassende Theorie gibt, die das komplette Relaxationsspektrum zusammenhängend beschreiben kann, gelten Modelle mit einer multidimensionalen Energielandschaft als vielversprechend [61, 62]. Das Glas wird als Potentialhyperfläche beschrieben, wobei die lokalen Minima den unterschiedlichen Glaszuständen zugeordnet werden. Abkühlen führt zur Absenkung der mittleren Teilchenenergie, s. d. die angrenzenden Maxima nicht mehr überwunden werden können und das System in einer Konfiguration, also in einem kleinen Teilbereich des vollen Phasenraums, „gefangen“ ist. Die Atome können ihrem Käfig von Nachbaratomen dann nur noch durch kollektive Sprungprozesse entkommen, an denen viele Atome beteiligt sind. Die Modenkopplungstheorie nach Götze und Sjögren [63, 64] sagt diesen Übergang des atomaren Transportmechanismus von lokalen Sprüngen ( $\alpha$-Relaxation) zur kollektiven Bewegung ( $\beta$-Relaxation) bei einer kritischen Temperatur $T_{C}>T_{g}$ voraus. Diese Theorie beschreibt den Glasübergang über rückgekoppelte Dichtekorrelationen (Moden) ausgehend von der Flüssigkeit. Unterhalb der kritischen Temperatur sind ein Teil der Moden eingefroren und das System wird nicht-ergodisch. 


\subsection{Polymere Gläser}

Das obige Modell ist auch auf Gläser aus komplexeren Molekülen, also z. B. Polymere anwendbar. Hier muss der mikroskopische Prozess, der zur makroskopischen Verformung der Probe führt, durch eine Bewegung ganzer Ketten oder Kettensegmente stattfinden, da die Ketten über chemische Bindungen zusammengehalten werden. Zwischen den einzelnen Makromolekülen wirken nur schwache Nebenvalenzbindungen (van der Waals Bindungen), die unter Kraftwirkung leicht aufgebrochen werden können. Unterhalb der Glastemperatur ist Verformung auch hier nur über kollektive Prozesse möglich, bei denen nur wenige Segmente involviert sind. Beispiele für solche Bewegungen, die eine Verlagerung des Schwerpunkts des Kettensegmentes bewirken, sind Rotationen, an denen nach Schatzki [65], Wunderlich [66] oder Boyer [67] jeweils 7, 6 oder 5 C-C-Bindungen beteiligt sind. Dabei sind stets zwei Bindungen kolinear ausgerichtet, s. d. die Rotationen mit einer Kurbelwellenbewegung beschrieben werden können.

Oberhalb der Glasübergangstemperatur ist die Bewegung durch Verschlaufungen, also temporäre Verhakungspunkte unterschiedlicher Makromoleküle, die die freie Bewegung der Kettensegmente behindern, bestimmt. Das Reptationsmodell nach deGennes [68] beschreibt die Bewegung einer Kette in einem Kanal aus umgebenden Molekülen. Bewegung ist hier nur in einer Dimension in Richtung des Kanals möglich. Entropiebedingt sind die einzelnen Segmente nicht gestreckt, sondern verknäult. Im Gleichgewicht existieren somit stets Schleifen ohne eingeschlossene andere Ketten, also Verhakungspunkte. Durch diese Schleifen kann „freie Länge“ gespeichert und analog zur Versetzungsbewegung im Kristall bewegt werden, s.d. dann Verformung möglich ist. Die Wiederherstellung der „Gleichgewichtslänge“ der Schleifen besitzt aber eine charakteristische Relaxationszeit, womit stets eine Zeitabhängigkeit vorhanden ist, die die rein elastische Verformung auch bei unvernetzten Polymeren ausschließt. Stattdessen beobachtet man viskoses, bzw. visko-elastisches Verhalten. Bei vernetzten Polymeren sind einzelne Ketten zusätzlich über atomare Bindungen, also feste Vernetzungspunkte verknüpft und ein rein viskoses Verhalten wird auch bei hohen Temperaturen unterdrückt [69].

Zusätzlich zum viskosen Fließen und zu den oben bereits diskutierten Relaxationsmoden gibt es in diesen komplexen Makromolekülen allerdings eine Vielzahl weiterer intrinsischer Freiheitsgrade, die zu stark lokalisierten Bewegungen führen. Durch die Komplexität der molekularen Struktur können zusätzliche Dreh-, Schwingungs- und Verschiebungsbewegungen statt- 
finden, durch die Energie dissipiert werden kann. Diese zusätzlich auftretenden Relaxationsmechanismen werden nach ihren charakteristischen Frequenzen sortiert $\beta$ - und $\gamma$-Relaxation genannt. Die Bezeichnung der verschiedenen Relaxationsprozesse ist in der Literatur somit leider nicht eindeutig und besonders im Vergleich mit den Nomenklaturen, die bei metallischen Glasbildnern verwendet werden, verwirrend. So ist der oben beschriebene slow- $\beta$ Prozess der metallischen Gläser keinesfalls mit dem mit Seitengruppenschwingung assoziierten $\beta$-Prozess bei Polymeren zu vergleichen, auch wenn beide ein ähnliches Temperaturverhalten zeigen. Bei den Polymeren spaltet der viskose Fließprozess je nach Kettenlänge des Materials zusätzlich in einen Prozess in dem nur Kettensegmente bewegt werden $(\alpha$ Relaxation, segmental mode) und einen langsameren Prozess, bei dem das Abgleiten ganzer Ketten beteiligt ist ( $\alpha^{\prime}$-Relaxation, normal mode), auf [39, 70]. Diese Prozesse sind je nach Zeitskala der Messung unterschiedlich gut voneinander zu trennen, da auch sie eine unterschiedliche Temperaturabhängigkeit der Relaxationszeiten zeigen.

Über die Kernresonanzspektroskopie ist es im Experiment möglich, den verschiedenen Relaxationsmoden mikroskopische Bewegungen zuzuordnen. Spiess et al. zeigten für die ersten beiden Mitglieder der Familie der Poly(alkyl methacrylat)e PMMA und PEMA ähnliches Verhalten, jedoch mit leichten Abweichungen oberhalb der Glasübergangstemperatur [71]: Bereits bei niedrigen Temperaturen wird für beide Polymere eine, wenn auch eingeschränkte, Mobilität der Hauptkette beobachtet. Diese Mobilität beruht auf Ausgleichs- und Kippschwingungsbewegungen, die Teile der Hauptkette aufgrund der bereits eingesetzten $\beta$-Relaxation durchlaufen müssen. Diese beinhaltet hauptsächlich eine Rotationsbewegung der gesamten Ester-Seitengruppe zwischen zwei um $180^{\circ}$ versetzte Hauptpositionen $\left(, 180^{\circ}\right.$-flips $\left.{ }^{\circ}\right)$. Aufgrund der unterschiedlichen Größen der Seitengruppen sind die Korrelationszeiten der $\beta$-Relaxation für beide Polymere etwa um eine Größenordnung verschieden, was zu einer unterschiedlichen Kopplung zwischen der $\alpha$ - und $\beta$-Relaxation führt. Diese Variation der Kopplung wird in Kapitel 6.6 in Bezug auf mechanische und dielektrische Messungen eingehend diskutiert. 


\section{Experimentelles}

Im Folgenden werden die im Rahmen dieser Arbeit verwendeten Herstellungs- und Charakterisierungsmethoden vorgestellt. Während die eher außergewöhnlichen und meist speziell hier in Göttingen aufgebauten Anlagen, besonders in den Bereichen der Dünnschichtherstellung und der Spektroskopie eingehend beschrieben werden, beschränkt sich die Darstellung der Standardmethoden auf eine kurze Beschreibung der verwendeten Apparaturen und der Angabe ihrer wichtigsten Kenndaten. Aufgrund ihrer zentralen Rolle in dieser Arbeit wird die Methode der Mechanischen Spektroskopie in einem eigenen Kapitel 4 abgehandelt.

\subsection{Gepulste Laserdeposition}

Unter den vielen Methoden der Dünnschichtherstellung ist die gepulste Laserdeposition (Pulsed Laser Deposition, PLD) wohl die Vielseitigste. Grundlegend bereits 1965 von Smith und Turner entwickelt [72], konnten seitdem die unterschiedlichsten Materialkassen mit ihrer Hilfe deponiert werden. Diese beschränken sich längst nicht mehr auf die HochtemperaturSupraleiter, durch die die Methode seit 1986 in vielen Gruppen zum Einsatz kam [73], sondern enthalten mittlerweile nahezu alle Materialklassen wie Metalle, Halbleiter und Keramiken [58-62].

Experimentell trifft zunächst ein gepulster Laserstrahl hoher Energie und Intensität auf ein in einer Vakuumkammer befindliches Festkörper-Target. Der größte Teil der Laserenergie wird absorbiert und bewirkt die thermische Verdampfung eines Teils der Targetoberfläche. Dieses verdampfte Material bildet vor dem Target ein quasi-zweidimensionales Plasma, welches durch den immer noch einfallenden Laserpuls weiter erhitzt und ionisiert wird. Bedingt durch Stoßprozesse breitet sich das Plasma in einer stark gebündelten Plasmakeule senkrecht vom Target aus. Da die Elektronen im Plasma aufgrund ihrer geringeren Masse deutlich schneller sind als die Ionen, entsteht eine Raumladungszone, durch die die zurückgebliebenen Ionen auf 
Energien bis zu $100 \mathrm{eV}$ beschleunigt werden [74]. Während das Plasma expandiert, kühlt es schnell ab und der Ionisationsgrad nimmt durch Rekombination ab. Erreicht das Material das gegenüberliegende Substrat, schlägt es sich auf diesem nieder und bildet die Schicht.

Ende der achtziger Jahre wurde durch Hansen und Robitaille mit den Polymeren eine weitere Materialklasse erschlossen [10]. Während der grundsätzliche Aufbau dem der Deposition von nicht-organischem Material entspricht, ist hier aufgrund der höheren Komplexität der zu übertragenden Moleküle der Ablations- und Depositionsmechanismus bedeutend komplizierter als der oben beschriebene und immer noch Gegenstand von wissenschaftlichen Diskussionen [75, 76]. Da in dieser Arbeit auch weiterführende Untersuchungen zu diesem Prozess gemacht wurden, findet sich eine eingehende Beschreibung in Kapitel 5, während hier nur der verwendete Aufbau beschrieben wird.

Als Energiequelle diente im Rahmen dieser Arbeit ein KrF-Excimer-Laser (LAMBDA PHYSIK LPX 110i) mit einer Wellenläge von 248 nm, einer Pulsdauer von 30 ns und einer Repetitionsfrequenz von $3 \mathrm{~Hz}$. Das als Pulver vorliegende Ausgangsmaterial wurde in einer knapp über die jeweilige Glastemperatur geheizten Presse bei $3 \cdot 10^{7} \mathrm{~N} / \mathrm{cm}^{2}$ zu klaren, festen Targetzylindern mit einem Durchmesser von 2,5 cm und wenigen Millimetern Höhe gepresst.

Als Substrate wurden neben den in Kapitel 4 beschriebenen Oszillatoren beidseitig polierte (111)-Silizium-Einkristalle verwendet. Für die Untersuchungen mit der Infrarotspektroskopie dienten aufgrund der höheren Reflektivität unpolierte Substrate aus technischem Aluminium.

Mit zwei unterschiedlichen Depositionskammern wurden auch zwei leicht unterschiedliche Depositionsgeometrien verwendet: Zum einen eine einfache fokussierende Geometrie, bei der der Fokus des gebündelten Laserstrahls auf der Targetoberfläche liegt, andererseits eine abbildende Geometrie, bei der das Laseraustrittsfenster verkleinert auf der Targetoberfläche abgebildet wird. Durch die Abbildung wird im Vergleich zur Fokussierung eine deutlich höhere Energiehomogenität im Brennfleck erreicht [77]. Da sich jedoch bei der Untersuchung von laserdeponierten Polymeren bis dato keine signifikanten Unterschiede in den Eigenschaften der hergestellten Filme finden lassen konnten, werden beide Geometrien im Folgenden als gleichwertig angenommen. Mit der typischen Pulsenergie von 60 mJ/Puls und einer Brennfleckgröße von $2 \mathrm{~mm}^{2}$ ergibt sich eine Laserfluenz von $3 \mathrm{~J} / \mathrm{cm}^{2}$. Um Effekte durch Targetalterung, also durch Oberflächenveränderung zu minimieren, wurde ein weitaus größerer Teil der Targetfläche benutzt. Dazu wurde einerseits eine azentrische Rotation der optischen Linse 
ausgenutzt, die den Brennfleck kreisförmig auf dem Target wandern lässt. Andererseits kann die Position des Targets über einen Schrittmotor verfahren werden, s. d. ein möglichst großer Bereich abgerastert wird. Dabei ist zu beachten, dass die verwendeten Polymere in Verbindung mit $248 \mathrm{~nm}$-Strahlung Inkubationseffekte aufweisen, wodurch der Abtragsprozess erst nach einigen 10 Pulsen stabil ist. Die Targets müssen also entsprechend vorbehandelt („,eingeschossen“) werden [78].

Der Target-Substrat-Abstand beträgt im Falle der fokussierenden Geometrie $40 \mathrm{~mm}$, in der abbildenden Geometrie ist er mit $54 \mathrm{~mm}$ etwas größer, was aber bis auf eine verringerte Depositionsrate keine Auswirkungen hat. Um einer Verunreinigung der Substrat- bzw. Filmoberfläche entgegen zu wirken, ist der Restdruck in der Vakuumkammer vor der Deposition stets besser als $5 \cdot 10^{-8}$ mbar. Während der Herstellung steigt er um bis zu vier Größenordnungen an, fällt danach jedoch sehr schnell wieder in den ursprünglichen Bereich ab.

\subsection{Rasterelektronenmikroskopie}

Zur Untersuchung der Oberflächenmorphologie wurden rasterelektronenmikroskopische Aufnahmen an zwei unterschiedlichen Geräten hergestellt. Für Standardaufnahmen wurde ein CAMBRIDGE InSTRUMENTS S360 mit Wolframkathode und Sekundärelektronendetektor benutzt. Die nicht leitenden Proben wurden dafür zunächst in einem BIO-RAD POLARON SEM Coating System mit einer etwa $20 \mathrm{~nm}$ dicken Goldschicht bedampft, um Aufladungseffekte zu vermeiden. Mit dem Leo SuPRA 35 (Feldemissionskathode und SE+ Inlens Detektor) konnten hochauflösende Bilder erstellt werden. Dieses Gerät erlaubte außerdem das Arbeiten mit sehr geringen Beschleunigungsspannungen, s. d. eine Beschädigung der Proben auf ein Minimum reduziert und teilweise auf die Goldschicht verzichtet werden konnte.

\subsection{Röntgendiffraktometrie}

Zur Analyse der Mikrostruktur der Filme wurde auf die Röntgenstreuung (X-Ray Diffraction $X R D$ ) zurückgegriffen, die z. B. bei Cullity ausführlich beschrieben ist [79]. Dazu wurde zum einen ein PHILIPS X'PERT Vierkreisdiffraktometer mit Co-Röhre verwendet.

Mit einem SIEMENS D500 Zweikreisdiffraktometer mit Co-Röhre war es zudem möglich, während der Röntgenmessung die Probentemperatur zu variieren und somit temperaturbe- 
dingte Mikrostrukturänderungen zu verfolgen. Hier befindet sich ein heizbarer Probenhalter in einer Vakuumkammer, die mit Vor- und Turbomolekularpumpe auf etwa $1 \cdot 10^{-6}$ mbar evakuiert werden kann. Der Röntgenstrahl gelangt durch ein Folienfenster in die Kammer, trifft auf die Probe und läuft dann durch ein weiteres Fenster zum Detektor. Ein programmierbarer EUROTHERM 2416 PID Regler steuert mit Hilfe eines Thermoelements den Heizstrom, der den im Probenhalter eingebauten Widerstandsofen temperiert. Über Rampenprogramme wird zunächst die jeweilige Solltemperatur angefahren. Nach kurzer Wartezeit zur Temperaturhomogenisierung wird dann ein Diffraktogramm aufgenommen und anschließend die nächste Temperatur angefahren. Der apparative Fehler der Probentemperatur während der Messung kann auf $\pm 3{ }^{\circ} \mathrm{C}$ abgeschätzt werden.

\subsection{Röntgenreflektometrie}

Die Schichtdicken sowie die Oberflächen- und Grenzflächenrauigkeiten dünner Filme mit geringer Rauigkeit lassen sich hervorragend mit der Röntgenreflektometrie (X-Ray Reflectometry $X R R$ ), also der spekulären Kleinwinkelröntgenstreuung untersuchen. Dazu wurde das auch für die Diffraktometrie verwendete PHILIPS X'PERT eingesetzt, hier allerdings mit einer Sekundäroptik mit zusätzlichem Monochromator. An die erhaltenen Reflektogramme wurden mit Hilfe des Softwarepakets IMD [80], bei dem der rekursive Parratt Algorithmus [81] verwendet wird, Simulationen angepasst.

\subsection{Fourier Transformations Infrarot-Spektroskopie}

Die Infrarotspektroskopie ist eine weit verbreitete Methode zur Untersuchung von chemischen Bindungsverhältnissen in Polymeren. Eingestrahltes infrarotes Licht regt im untersuchten Material Eigenmoden der Molekülschwingungen an und wird somit in seiner Intensität geschwächt. Aus der spektralen Lage der Absorptionsbanden lassen sich dann Rückschlüsse auf die chemischen Bindungsverhältnisse ziehen. Eine gute Einführung in die Methode findet sich bei Günzler [82]. Hier wurde ein BRUKER TENSOR 37 mit LN $\mathrm{N}_{2}$-gekühltem MCT-Detektor (HgCdTe) und computergesteuerter Winkelverfahreinheit HARRICK AUTOSEAGUL (Einfallswinkel zur Oberfläche $54^{\circ}$ ) verwendet. Die Spektren wurden an auf rauen AluminiumSubstraten hergestellten Filmen in Reflektion mit einer Auflösung von $4 \mathrm{~cm}^{-1}$ gemessen. Charakteristika des Substrats, der Umgebung und der Apparatur konnten durch die Korrektur der 
Spektren um zuvor aufgenommene Hintergrundmessungen eliminiert werden. Um den Einfluss von Absorptionsbanden des Wassers zu reduzieren, wurde der Spektrometerraum vor und während der Messung mit getrockneter Luft gespült.

\subsection{Größenausschluss-Chromatographie}

In Zusammenarbeit mit dem Institut für Physikalische Chemie der Universität Göttingen wurde die Molmassenverteilung ausgesuchter Proben mit der Größenausschluss-Chromatographie (Size Exclusion Chromatography SEC) untersucht. Diese Methode beruht auf der Trennung der gelösten Moleküle durch sterischen Ausschluss in einem porösen Trenngel [3] und liefert über die Verweilzeit des gelösten Polymers im Trenngel Informationen über das Volumen der Makromoleküle und somit über die Kettenlänge linearer Polymere.

\subsection{Dielektrische Verlustspektroskopie}

Der breite Frequenzbereich der Relaxationsdynamik polymerer Materialien lässt sich mittels dielektrischer oder Impedanz-Spektroskopie vermessen. Basierend auf der Wechselwirkung eines externen elektrischen Feldes mit dem internen elektrischen Dipolmoment der Probe können Polarisationen durch induzierte Momente bzw. durch die Ausrichtung permanenter Momente bestimmt und mit der Dynamik auf molekularer Ebene in Verbindung gebracht werden. Im Rahmen dieser Arbeit wurde um einen HEWLETT-PACKARD 4192A Impedance Analyser eine Anlage aufgebaut, mit der im Frequenzbereich von $5 \mathrm{~Hz}$ bis $13 \mathrm{MHz}$ temperaturabhängig Dünnschichtproben untersucht werden können.

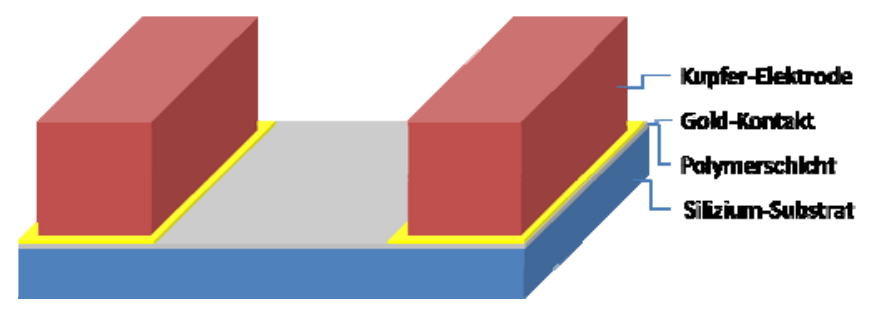

Abbildung 3.1: Schematische Messgeometrie für die dielektrischen Messungen. Die Kupfer-Elektroden werden über geklemmte Koaxialkabel mit dem Analysator verbunden.

Für die Messungen wurden Schichten auf 11x11 mm² großen, (111)-orientierten SiliziumSubstraten verwendet, auf die mittels einer Maske und eines handelsüblichen Sputtercoaters (BIO-RAD Polaron SEM Coating System) im Abstand von $5 \mathrm{~mm}$ zwei $50 \mathrm{~nm}$ dicke Gold- 
kontakte aufgebracht wurden. Auf diese Kontakte wurde mit einer Spiralfeder jeweils ein massiver 10x2 mm $\mathrm{mm}^{2}$ großer Kupferstempel gepresst, von denen dann geschirmte Koaxialkabel zum Analysator geführt wurden (s. Abbildung 3.1). Unter Annahme einer parallelen Ersatzschaltung von Widerstand und Kondensator wurde dann der Betrag des Leitwerts $Y_{a b s}$ und der Phasenverschiebung zwischen Strom und Spannung gemessen. In der planaren Geometrie bestimmt sich dann der Leitwert unabhängig von der Schichtdicke als Quotient von $Y_{a b s}$ und dem Abstand der Kontakte. Aus den Messwerten ergeben sich dann mit der Dielektrizitätskonstante $\varepsilon_{0}=8,85 \cdot 10^{-12} \mathrm{As} / \mathrm{Vm}$ mit

$$
\varepsilon^{\prime}(\omega)=\frac{\sin \delta \cdot Y_{a b s}}{\omega \cdot \varepsilon_{0}} \text { und } \varepsilon^{\prime \prime}(\omega)=\frac{\cos \delta \cdot Y_{a b s}}{\omega \cdot \varepsilon_{0}}
$$

die Real- und Imaginärteile der dielektrischen Permittivität $\varepsilon(\omega)=\varepsilon^{\prime}(\omega)$ - i $\varepsilon^{\prime \prime}(\omega)$. Um den eigentlichen dielektrischen Verlust zu bestimmen, wird die imaginäre Permittivität um einen Gleichspannungsanteil $Y_{0}$, bestimmt aus dem Mittelwert der Leitwerte für Frequenzen zwischen 10 und $20 \mathrm{~Hz}$, korrigiert:

$$
\varepsilon_{\text {korr }}^{\prime \prime}(\omega)=\frac{(\cos \delta \cdot Y)-Y_{0}}{\omega \cdot \varepsilon_{0}}
$$

Im Probenhalter sind Heizdrahtwicklungen eingelassen, s. d. Temperaturen zwischen Raumtemperatur und $500 \mathrm{~K}$ realisiert werden können ${ }^{1}$. Die Temperaturregelung übernimmt ein EUROTHERM 3508 PID Regler in Verbindung mit einem KNÜRR-HEINZINGER POLARIS 35-20 Netzteil. Zur Vermeidung von Verunreinigungen ist der komplette Probenbereich in eine Vakuumkammer integriert, die mit einer Rotationspumpe auf etwa $1 \cdot 10^{-2}$ mbar evakuiert werden kann. Die komplette Prozessführung, Messwertaufnahme und Synchronisation wurde mit einem LABVIEW Programm realisiert.

\subsection{Finite-Elemente-Modellierung}

Als numerisches Verfahren zur Lösung von Systemen aus partiellen Differentialgleichungen mit Randbedingungen eignet sich die Finite-Elemente-Methode (FEM) sehr gut zur nähe-

\footnotetext{
${ }^{1}$ Prozessführung in Rampen mit einer Heizrate von $2 \mathrm{~K} / \mathrm{min}$ und Haltezeiten während der Messung.
} 
rungsweisen Lösung vielfältiger physikalischer Fragestellungen. Dazu wird zunächst das Berechnungsgebiet in eine beliebig große Anzahl finiter Elemente eingeteilt (,,meshing“). Für jedes dieser finiten Elemente werden Ansatzfunktionen definiert, die zusammen mit den Anfangs-, Rand- und Übergangsbedingungen ein Gleichungssystem bilden, welches numerisch gelöst werden kann. Im Rahmen dieser Arbeit wurden die Module „Heat Transfer" und „Structural Mechanics“ des Programms COMSOL MulTIPHYSICS in der Version 3.5.0.608 verwendet, um zum einen die experimentell schwer zugänglichen Temperaturverteilungen in den Oszillatoren zu bestimmen. Zum anderen konnte die FEM genutzt werden, um die unterschiedlichen Schwingungsmoden der Doppelpaddeloszillatoren zu charakterisieren. Die verwendeten Materialkonstanten wurden der programminternen Bibliothek entnommen. 


\section{Mechanische Spektroskopie}

Analysiert man die Verknüpfung interner mikroskopischer Prozesse mit externen makroskopischen Materialeigenschaften, drängt sich die mechanische Spektroskopie als Methode der Wahl auf. So dient sie nicht nur zur dynamischen Bestimmung komplexer elastischer Kenngrößen wie Elastizitäts- und Schermodul, sondern kann auch über Relativmessungen, z. B. der Dämpfung als Funktion der Temperatur ein äußerst empfindlicher Indikator für Umwandlungen und Gefügeänderungen im Material sein. Grundlegende Idee dabei ist, dass die makroskopische Anregung bei gegebener Frequenz und Temperatur eine Antwort des Materials hervorruft. Diese Antwort und damit die Dämpfung des Materials ist besonders stark, wenn die äußere Anregung auf eine Resonanz der inneren mikroskopischen Prozesse stößt, wenn also die inneren Schwingungen mit der äußeren Frequenz schwingen können. Die Dämpfung beruht dabei auf der Dissipation von Energie im Material durch innere Reibung, in der klassischen Metallphysik zum Beispiel durch die Bewegung von Defekten (Fremdatome, Versetzungen, Grenzflächen usw.) [83-88]. Bei komplexeren Materialien, wie z. B. metallischen oder polymeren Gläsern ist die Identifikation der jeweiligen Dämpfungsmechanismen schwieriger. Gelingt es jedoch, so lässt sich dies zur empfindlichen Untersuchung einer Vielzahl von mikroskopischen Eigenschaften und Strukturen bis hinunter zu atomaren Dimensionen ausnutzen.

Möglichst allgemein formuliert ist die mechanische Spektroskopie die Messung der Zeitabhängigkeit mechanischen Verhaltens. Dabei sind der Zeitabhängigkeit kaum Grenzen gesetzt: Das Spektrum umfasst von langsamsten Kriechversuchen über Resonanzschwingungen bis in den Ultra- und Hyperschallbereich mehr als 15 Dekaden. Die Anwendungen limitiert lediglich die Ausnahme von Prozessen, die zu einer bleibenden Formänderung führen, stattdessen sind elastische Eigenschaften und im Besonderen die Abweichungen von der idealen Elastizität (Anelastizität, Viskoelasitizität, elastische Plastizität) [89-91] im Fokus der mechanischen Spektroskopie. Im Speziellen beschäftigt sie sich vorwiegend mit dem Materialverhalten unter periodischer mechanischer Beanspruchung bei erzwungenen und freien Schwingungen. Im 
Bereich der akustischen Frequenzen kommt dabei Resonanzmethoden, die auch in dieser Arbeit verwendet werden, die größte Bedeutung zu. Experimentell misst man dabei die Eigenfrequenz und die Dämpfung mechanischer Schwingungen. Da in dieser Arbeit Dünnschichten untersucht werden, ist der Zugang über einen massiven Oszillator, der nur aus dem zu messenden Material aufgebaut ist, nicht möglich. Stattdessen werden Oszillatoren mit möglichst geringer Eigendämpfung verwendet und diese dann mit dem Probenmaterial beschichtet. Die zusätzliche Masse vergrößert die Trägheit des Gesamtsystems und die deponierte Schicht wird Teil der schwingenden Bestandteile, was die rücktreibenden Momente der Schwingung verändert. Beide Effekte verursachen eine Verschiebung der Resonanzfrequenzen. Aus der Differenz der Messdaten des beschichteten und des unbeschichteten Oszillators ergibt sich der Beitrag der dünnen Schicht. Über die temperaturabhängige Messung dieser Verschiebung der Resonanzfrequenzen und der Änderung der Dämpfung lassen sich dann die temperaturabhängigen Elastizitäts- bzw. Schermoduli der deponierten Schichten bestimmen.

In dieser Arbeit wurden zwei verschiedene Typen von Oszillatoren verwendet, deren Merkmale und besondere Vorzüge im Folgenden dargestellt werden.

\subsection{Der Doppelpaddeloszillator (DPO)}

Der Doppelpaddeloszillator ist ein mechanischer Oszillator mit besonders hoher Güte, dessen Form bereits 1985 von Kleiman et al. vorgeschlagen wurde [92, 93]. Abbildung 4.1 zeigt die spezifische Geometrie des Doppelpaddeloszillators mit Kopf, Hals, Flügeln, Bein und Fuß. Die in dieser Arbeit verwendeten DPOs wurden aus $300 \mu \mathrm{m}$ dicken, (100)-orientierten Silizium-Wafern hoher Reinheit und geringer Dotierung präpariert (CRYSTEC Berlin; spezifischer Widerstand bei Raumtemperatur größer als $5 \mathrm{kOhm} \mathrm{cm}$ ).

Durch die kleine Zahl an Fremdatomen soll der Effekt innerer Defekte minimiert und somit eine möglichst geringe Eigendämpfung sichergestellt werden. Im Rahmen dieser Untersuchungen wurde jedoch keine signifikante Abhängigkeit der Schwingungseigenschaften von der Dotierung des Siliziums festgestellt. Während in früheren Arbeiten sowohl durch Photolithographie und Ätz- als auch durch Laserprozesse präparierte Oszillatoren verwendet wurden [94], wurden alle hier verwendeten Oszillatoren in Zusammenarbeit mit dem Fraunhofer Institut für Lasertechnik in Aachen durch Laserschneiden hergestellt. Dabei schneidet ein gepuls- 
ter Laserstrahl eines Nd:YAG-Lasers vom Typ LASAG KLS246-040 FC ${ }^{1}$ jeweils vier DPOs aus einem vier Zoll großen Si-Wafer aus.

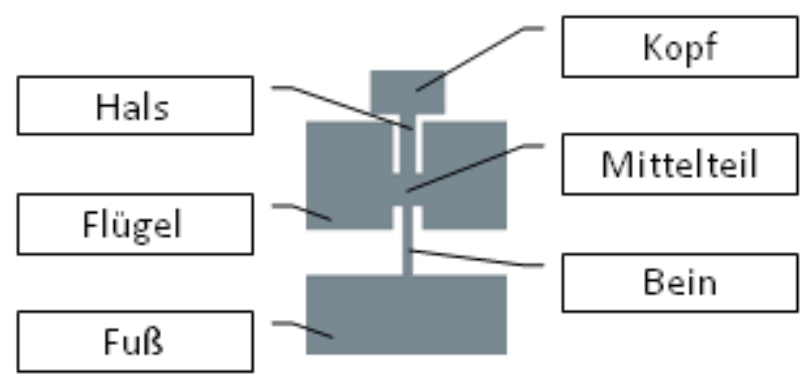

Abbildung 4.1: Maßstabstreue Geometrie des Doppelpaddeloszillators. Die Gesamthöhe des Oszillators aus einkristallinem Silizium beträgt $28,58 \mathrm{~mm}$, die Dicke $300 \mu \mathrm{m}$.
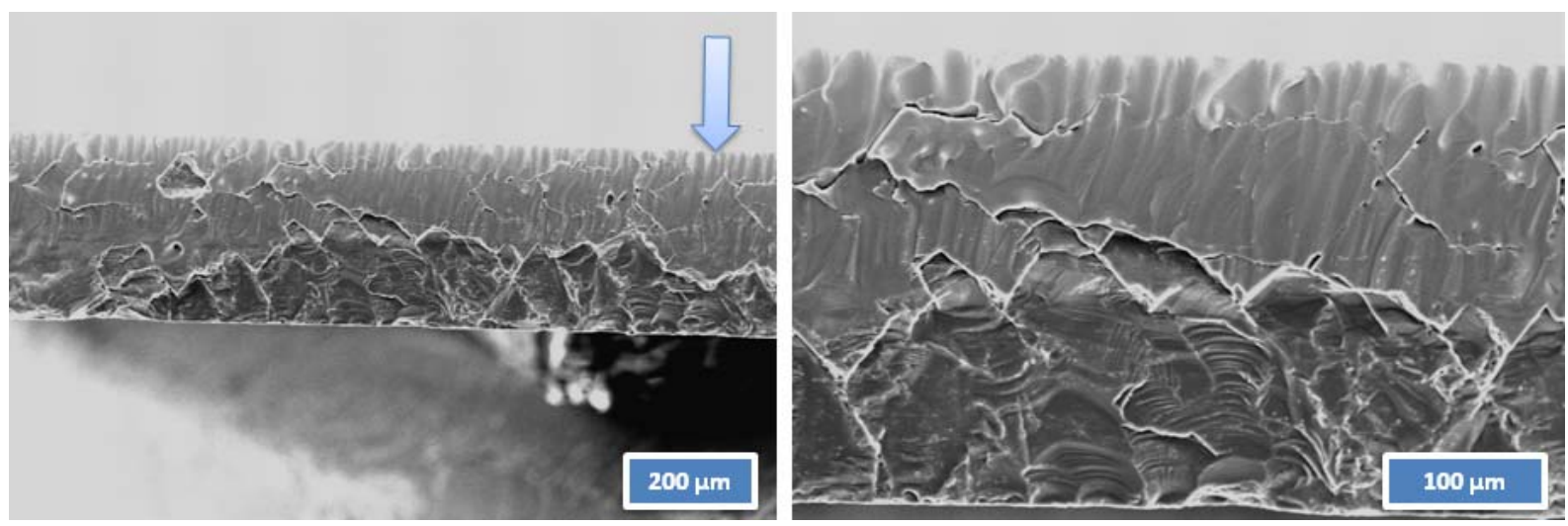

Abbildung 4.2: Rasterelektronenmikroskopische Aufnahmen der Schnittkante eines lasergeschnittenen Doppelpaddeloszillators. Der Pfeil im linken Bild markiert die Einfallsrichtung des Laserstrahls. Die Schnittkante weist eine sehr raue und zerklüftete Oberfläche auf und lässt zwei Bereiche erkennen: Im oberen Bereich wurde das Material durch den Laser aufgeschmolzen, vom Prozessgas ausgeworfen und sauber geschnitten, während in der unteren Hälfte gröBere Siliziumstücke herausgebrochen wurden.

Die Schnittkanten der DPOs stehen nach dem Schneiden sauber und senkrecht auf der PaddelEbene, s.d. der gesamte Oszillator einen rechteckigen Querschnitt aufweist. Wie in Abbildung 4.2 dargestellt, sind die Kanten allerdings durch das lokale Aufschmelzen und Wiedererstarren extrem aufgeraut und zerklüftet. Vermutlich ist oberflächennah auch die einkristalline Struktur des Siliziums zerstört worden. Rösner fand beim Vergleich von lasergeschnittenen und geätzten DPOs stets leicht erhöhte Dämpfung bei den lasergeschnittenen Oszillatoren und führte dies auf Energiedissipation in diesen gestörten Randbereichen zurück [94]. Die nach dem Schneideprozess stark verschmutzen DPOs müssen vor dem Einbau in die

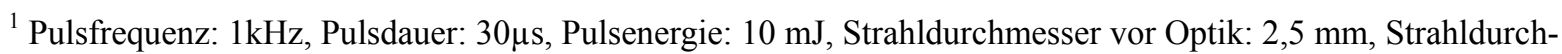
messer am Wafer: $25 \mu \mathrm{m}$, Prozessgas: Stickstoff
} 
Messapparatur gereinigt werden. Dazu wird zunächst mit einem Glasfaserpinsel das an den Kanten aufgeschmolzene und wieder erstarrte Silizium vorsichtig abgefegt. Daraufhin wird das DPO mit einem Teflon-Band am Hals aufgehängt und nacheinander mehrmals in Aceton und Methanol im Ultraschallbad gereinigt.

\subsubsection{Schwingungsverhalten}

Abbildung 4.3 zeigt das mit der in Kapitel 4.1.2 beschriebenen Anlage über einen Lichtzeiger am Paddelkopf aufgenommene Modenspektrum des Doppelpaddeloszillators. In der Auftragung ist zwischen der Amplitude in vertikaler Richtung (oben) und horizontaler (unten) Richtung der Schwingung zu unterscheiden. So kann bereits qualitativ zwischen Biegeschwingungen (CL: „CantiLever“ Schwingungen) und Torsionsschwingungen (ST: Symmetrische Torsion und AS: Asymmetrische Torsion) unterschieden werden. Während die Torsionsmoden aufgrund geringen Ausschlags nur eine kleine Auslenkung des Lichtzeigers bewirken und somit nur bei passend ausgerichtetem Detektor nachzuweisen sind, ist die durch die Biegeschwingungen hervorgerufene Auslenkung des Lichtzeigers größer. Somit kann diese Art der Schwingung auch auf der „falschen“ Detektorachse nachgewiesen werden, da die Projektion auf diese immer noch ein hinreichend großes Signal liefert. Die genaue Form der Schwingung ist experimentell z. B. über Laser-Doppler-Vibrometrische Experimente zugänglich [95].

Im Rahmen dieser Arbeit sind die den unterschiedlichen Moden zugehörigen Auslenkungen über FEM-Rechnungen bestimmt worden und in Abbildung 4.4 dargestellt. Farblich kodiert ist dabei als skalare Vergleichsgröße für die Spannung die von-Mises-Spannung eingetragen. Diese dient hier jedoch nur der qualitativen Veranschaulichung der Orte der größten Verspannung. Mit steigender Frequenz lassen sich folgende Schwingungsmoden erkennen: Bei $245 \mathrm{~Hz}$ liegt die erste Biegeschwingung CL1 vor, bei der zum Großteil das Bein verformt wird. Ebenso bei $455 \mathrm{~Hz}$, hier erfolgt die Verformung aber durch eine Verdrillung des Beines. Kopf und Flügel tordieren ohne Verdrehung des Halses gemeinsam um das Bein (Symmetrische Torsion ST). An der zweiten und dritten Biegeschwingung (CL2 und CL3) bei 1355 bzw. $2630 \mathrm{~Hz}$ sind sowohl das Bein, als auch Kopf und Mittelteil beteiligt. Die asymmetrischen Torsionsmoden AS1 und AS2 bei 4055 bzw. 4590 Hz werden durch eine Verdrillung des Halses bestimmt, das Bein bleibt dabei starr. Die Flügeloberseiten schwingen bei der AS1 in Phase mit dem Kopf, während sie bei der AS2 in Gegenphase schwingen, was zu einer bedeutend geringeren Verformung am Mittelteil führt. 


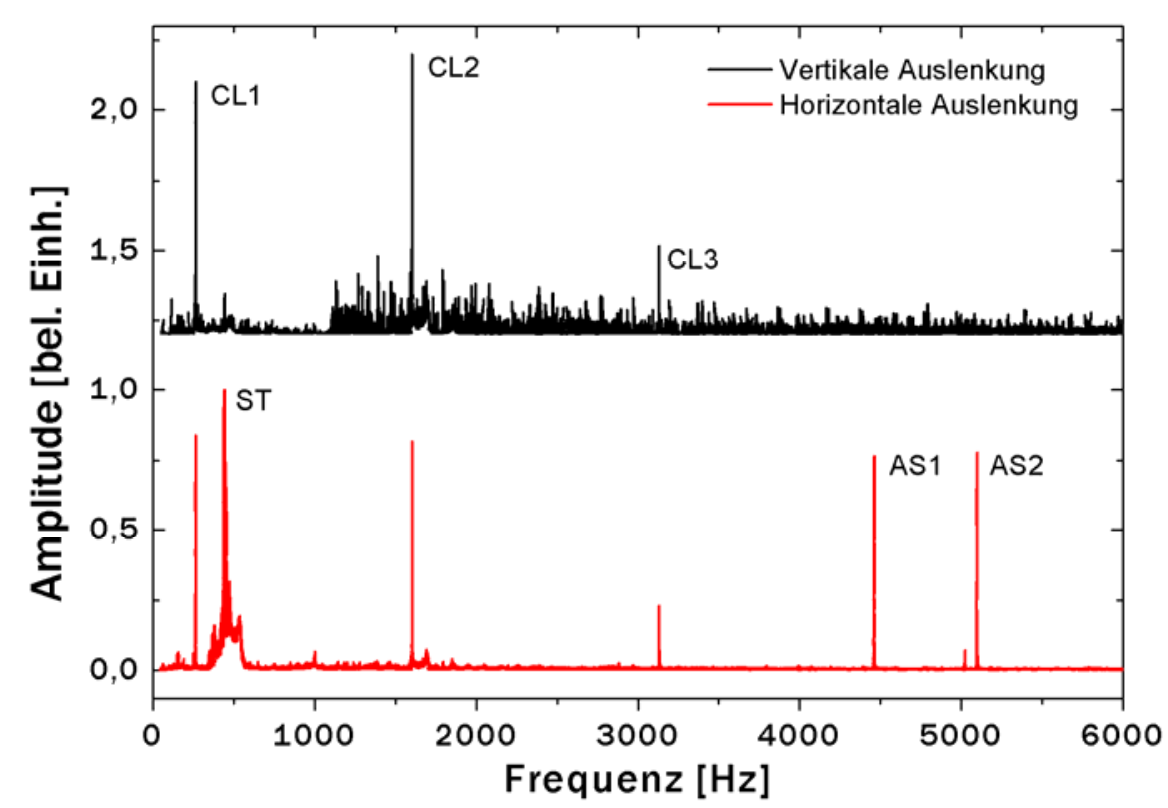

Abbildung 4.3: Modenspektrum eines Doppelpaddeloszillators. Die Schwingung wurde über einen auf den Paddelkopf gerichteten Lichtzeiger ausgelesen. Die obere Kurve zeigt die Auslenkung in vertikaler Richtung, also besonders die Biegeschwingungen. Die untere Kurve zeigt zusätzlich die Torsionsschwingungen. Das Auftreten von Biegeanteilen in der unteren Kurve lässt sich durch eine leichte Verkippung des Detektors aus der Horizontalen erklären. Die Kurven sind zur besseren Übersichtlichkeit gegeneinander verschoben.
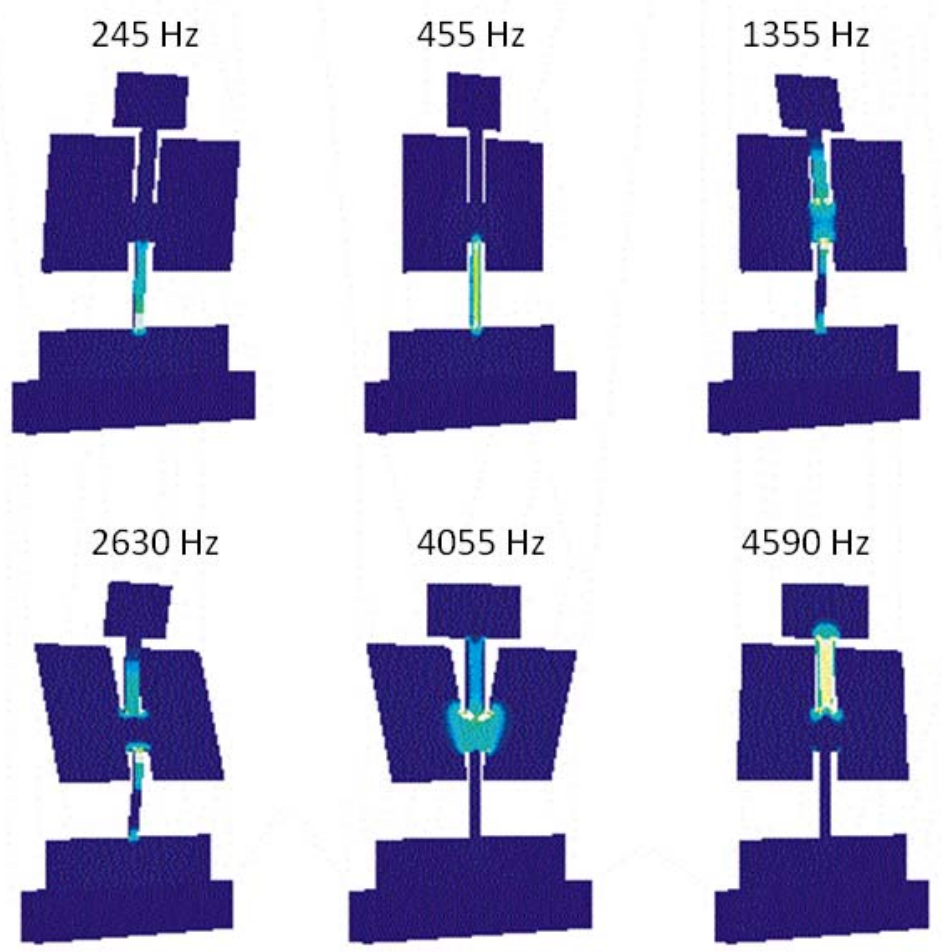

Abbildung 4.4: Mittels FEM bestimmte Resonanzfrequenzen des Doppelpaddeloszillators. Zur Veranschaulichung der Gebiete größter Verformung ist die van-Mises-Spannung als farbliche Kodierung eingetragen. 
Zur mathematischen Beschreibung der Torsionsschwingungen des Oszillators kann ein System aus zwei gekoppelten Drehschwingungssystemen angenommen werden, zum einen aus dem um den Hals tordierenden Kopf, zum anderen aus den um das Bein tordierenden Flügeln bestehend. Unter Vernachlässigung von Energieverlusten durch Dämpfung kann dieses System mit zwei gekoppelten Differentialgleichungen beschrieben werden:

$$
\begin{gathered}
I_{\text {Flïgel }} \ddot{\varphi}_{\text {Flïgel }}+\kappa_{\text {Bein }} \varphi_{\text {Fligel }}+\kappa_{\text {Hals }}\left(\varphi_{\text {Flïgel }}-\varphi_{\text {Kopf }}\right)=0 \\
\text { und } I_{\text {Kopf }} \ddot{\varphi}_{\text {Kopf }}+\kappa_{\text {Hals }}\left(\varphi_{\text {Kopf }}-\varphi_{\text {Flügel }}\right)=0 .
\end{gathered}
$$

Formel 4.2

Dabei beschreiben $I_{\text {Flügel }}$ und $I_{\text {Kopf }}$ die Trägheitsmomente von Flügeln und Kopf, $\varphi_{x}$ die jeweilige Auslenkung aus der Ruhelage und $\kappa_{x}$ die jeweiligen Winkelrichtgrößen. Mit dem Lösungsansatz

$$
\varphi_{\text {Flïgel }}=A_{\text {Flïgel }} \cdot e^{i \omega t} \text { und } \varphi_{\text {Kopf }}=A_{\text {Kopf }} \cdot e^{i \omega t}
$$

ergeben sich als Lösungen für die Eigenfrequenzen des gekoppelten Systems:

$$
\begin{aligned}
& \omega_{ \pm}^{2}=\frac{1}{2 I_{\text {Flügel }} I_{\text {Kopf }}} \cdot I_{\text {Kopf }}\left(\kappa_{\text {Bein }}+\kappa_{\text {Hals }}\right)+I_{\text {Flïgel }} \kappa_{\text {Hals }} \\
& \pm \frac{1}{2 I_{\text {Flugel }} I_{\text {Kopf }}} \cdot \sqrt{\left(I_{\text {Kopf }}\left(\kappa_{\text {Bein }}+\kappa_{\text {Hals }}\right)+I_{\text {Fligel }} \kappa_{\text {Hals }}\right)^{2}-4 I_{\text {Fligel }} I_{\text {Kopf }} \kappa_{\text {Bein }} \kappa_{\text {Hals }}} \text { Formel } 4.4
\end{aligned}
$$

Während die negative Lösung die symmetrische Torsionsmode ST beschreibt, handelt es sich bei der positiven Lösung um die antisymmetrische Torsionsmode AS2. Die Interpretation als AS1-Schwingung kann ausgeschlossen werden, da das starke Flattern der Flügel, welches bei dieser Mode zusätzlich auftritt, in der obigen Modellierung nicht berücksichtigt wurde. Mit Kenntnis der Trägheitsmomente und Winkelrichtgrößen lassen sich somit die Eigenfrequenzen der Schwingung bestimmen. Das Trägheitsmoment des Kopfes hängt von der Breite $b$, der Höhe $h$, der Dicke $t$ und der Dichte $\rho$ ab:

$$
I_{\text {Kopf }}=\frac{1}{12} \rho t h b^{3}
$$


Mit dem Satz von Steiner kann das Trägheitsmodul der Flügelpartie beschrieben werden. Es ergibt sich $I_{\text {Kopf }}=1,22 \cdot 10^{-10} \mathrm{~kg} \mathrm{~m}^{2}$ und $I_{\text {Flügel }}=5,93 \cdot 10^{-9} \mathrm{~kg} \mathrm{~m}^{2}$. Die Winkelrichtgrößen von Hals und Bein ergeben sich laut [96] mit dem Schermodul in axialer Richtung $G$ gemäß

$$
\begin{gathered}
\kappa=\frac{\beta b t^{3} G}{h} \\
\operatorname{mit} \beta=\frac{1}{3}\left(1-\frac{192}{\pi^{5}} \cdot \frac{t}{b} \cdot \sum_{n=1,3,5, \ldots}^{\infty} \frac{1}{n^{5}} \tanh \left(\frac{n \pi b}{2 t}\right)\right)
\end{gathered}
$$

analytisch $\mathrm{zu} \kappa_{\text {Hals }}=8,66 \cdot 10^{-2} \mathrm{Nm}$ und $\kappa_{\text {Bein }}=3,45 \cdot 10^{-2} \mathrm{Nm}$. Setzt man diese Daten oben ein, ergeben sich die (Kreis-)Frequenzen:

$$
\begin{gathered}
\omega_{S T}=2,39 \cdot 10^{3} \mathrm{~s}^{-1} \text { bzw. } f_{S T}=380 \mathrm{~Hz} \\
\omega_{A S 2}=2,62 \cdot 10^{4} \mathrm{~s}^{-1} \text { bzw. } f_{A S 2}=4170 \mathrm{~Hz} .
\end{gathered}
$$

Formel 4.8

Formel 4.9

Der Vergleich mit den gemessenen Resonanzfrequenzen in Abbildung 4.3 und den über die Simulation bestimmten Werten in Abbildung 4.4 zeigt, dass die errechneten Werte, trotz der vielen Näherungen, mit den tatsächlichen Werten gut übereinstimmen.

Um den Einfluss einer zusätzlichen dünnen Schicht auf dem Doppelpaddeloszillator zu bestimmen, geht man zunächst von den Resonanzfrequenzen der ungedämpften, ungekoppelten Einzelschwingungen aus:

$$
\omega_{\text {Kopf ,Hals }}=\sqrt{\frac{\kappa_{\text {Hals }}}{I_{\text {Kopf }}}} \text { und } \omega_{\text {Fligel,Bein }}=\sqrt{\frac{\kappa_{\text {Bein }}}{I_{\text {Fligel }}}} \text {. }
$$

Eine zusätzliche dünne Schicht ändert sowohl das Trägheitsmoment, als auch die Torsionskonstante des Oszillators, dann gilt für die ungekoppelte Schwingung des Kopfes um den Hals:

$$
\omega_{\text {Kopf }, \text { Hals }}+\Delta \omega_{\text {Kopf }, \text { Hals }}=\sqrt{\frac{\kappa_{\text {Hals }}+\Delta \kappa_{\text {Hals }}}{I_{\text {Kopf }}+\Delta I_{\text {Kopf }}}} .
$$


Erweiterung mit $\frac{1}{\omega_{\text {Kopf ,Hals }}}=\sqrt{\frac{I_{\text {Kopf }}}{\kappa_{\text {Hals }}}}$, Umstellen und Taylorentwicklung erster Ordnung liefert für kleine Änderungen $\Delta I_{\text {Kopf }}$ und $\Delta \kappa_{\text {Hals }}$ :

$$
\begin{aligned}
& 1+\frac{\Delta \omega_{\text {Kopf }, \text { Hals }}}{\omega_{\text {Kopf }, \text { Hals }}}=\sqrt{\frac{\kappa_{\text {Hals }}+\Delta \kappa_{\text {Hals }}}{I_{\text {Kopf }}+\Delta I_{\text {Kopf }}}} \cdot \sqrt{\frac{I_{\text {Kopf }}}{\kappa_{\text {Hals }}}} \\
& =\left(1+\frac{\Delta \kappa_{\text {Hals }}}{\kappa_{\text {Hals }}}\right)^{1 / 2}\left(1+\frac{\Delta I_{\text {Kopf }}}{I_{\text {Kopf }}}\right)^{-1 / 2} \\
& \approx\left(1+\frac{1}{2} \frac{\Delta \kappa_{\text {Hals }}}{\kappa_{\text {Hals }}}\right)\left(1-\frac{1}{2} \frac{\Delta I_{\text {Kopf }}}{I_{\text {Kopf }}}\right) \\
& \approx 1+\frac{1}{2} \frac{\Delta \kappa_{\text {Hals }}}{\kappa_{\text {Hals }}}-\frac{1}{2} \frac{\Delta I_{\text {Kopf }}}{I_{\text {Kopf }} .}
\end{aligned}
$$

Formel 4.12

Alternativ zu Formel 4.4 lassen sich die Resonanzfrequenzen der symmetrischen und antisymmetrischen Lösung des gekoppelten Systems mit $\mu=I_{\text {Kopf }} / I_{\text {Flügel }}$ beschreiben als:

$$
\omega_{A S 2}=\omega_{\text {Kopf }, \text { Hals }}(1+\mu) \text { und } \omega_{S T}=\omega_{\text {Flügel }, \text { Bein }}(1-\mu)
$$

Formel 4.13

Für die AS2 Mode gilt dann mit $\omega_{A S 2}+\Delta \omega_{A S 2}=\left(\omega_{\text {Kopf , Hals }}+\Delta \omega_{\text {Kopf , Hals }}\right) \cdot(1+\mu)$ für die Verschiebung der Resonanzfrequenz:

$$
\frac{\Delta f_{A S 2}}{f_{A S 2}}=\frac{\Delta \omega_{A S 2}}{\omega_{A S 2}}=\frac{\Delta \omega_{\text {Kopf }, \text { Hals }}}{\omega_{\text {Kopf }, \text { Hals }}} \approx \frac{1}{2} \frac{\Delta \kappa_{\text {Hals }}}{\kappa_{\text {Hals }}}-\frac{1}{2} \frac{\Delta I_{\text {Kopf }}}{I_{\text {Kopf }}}
$$

Über die totalen Differentiale der zusätzlichen Beiträge von Trägheitsmoment und Torsionskonstante nach der Schichtdicke:

$$
\Delta I_{\text {Kopf }}=\frac{d I_{\text {Kopf }}}{d t} \Delta t \text { und } \Delta \kappa_{\text {Hals }}=\frac{d \kappa_{\text {Hals }}}{d t} \Delta t
$$

ergeben sich mit den Ableitungen von Formel 4.5 und Formel 4.6 nach der Schichtdicke:

$$
\frac{d I_{\text {Kopf }}}{d t}=\frac{1}{12} \rho h_{\text {Kopf }} b_{\text {Kopf }}^{3} \text { und } \frac{d \kappa_{\text {Hals }}}{d t}=\frac{3 \beta b_{\text {Hals }} t^{2} G}{h_{\text {Hals }}}
$$


die dickenabhängigen Beiträge der dünnen Schicht zu Trägheitsmoment und Torsionskonstante:

$$
\Delta I_{\text {Kopf }}=\frac{1}{12} \rho_{\text {Film }} h_{\text {Kopf }} b_{\text {Kopf }}^{3} t_{\text {Film }} \text { und } \Delta \kappa_{\text {Hals }}=\frac{3 \beta b_{\text {Hals }} t^{2} G_{\text {Film }} t_{\text {Film }}}{\kappa_{\text {Hals }}} . \quad \text { Formel 4.17 }
$$

Da Schicht und Paddel bis auf die unterschiedliche Dicke gleiche Abmessungen besitzen, ergeben sich für die relativen Änderungen:

$$
\frac{\Delta I_{\text {Kopf }}}{I_{\text {Kopf }}}=\frac{\rho_{\text {Film }} t_{\text {Film }}}{\rho_{D P O} t_{D P O}} \text { und } \frac{\Delta \kappa_{\text {Hals }}}{\kappa_{\text {Hals }}}=\frac{3 G_{\text {Film }} t_{\text {Film }}}{G_{D P O} t_{D P O}} .
$$

Zusammen mit Formel 4.14 ergibt sich die Verschiebung der Resonanzfrequenz der AS2 Mode zu:

$$
\frac{\Delta f_{A S 2}}{f_{A S 2}}=\underbrace{\frac{3 G_{F i l m} t_{F i l m}}{2 G_{D P O} t_{D P O}}}_{\text {elastischer Anteil }}-\underbrace{\frac{\rho_{\text {Film }} t_{F i l m}}{2 \rho_{D P O} t_{D P O}}}_{\text {Masseanteil }} .
$$

Man erkennt, dass hier zwei Summanden unterschiedlichen Vorzeichens konkurrieren. Der elastische Anteil, der im Wesentlichen durch die Schermoduli von Schicht und DPO bestimmt ist, beschreibt, dass nun auch elastische Energie in der dünnen Schicht gespeichert wird. Dadurch erscheint der tordierende Hals härter, die Resonanzfrequenz wird also zu größeren Werten verschoben. Der Masseanteil beschreibt den Beitrag der zusätzlichen Masse auf dem Oszillator. Das gesamte System wird somit träger und die Resonanzfrequenz wird zu kleineren Werten verschoben ${ }^{1}$. Bei bekannter Schichtdicke und -dichte lässt sich der Schermodul der dünnen Schicht schließlich aus der Verschiebung der Resonanzfrequenz bestimmen:

$$
G_{F i l m}=\frac{2}{3} \frac{G_{D P O} t_{D P O}}{t_{F i l m}}\left(\frac{\Delta f_{A S 2}}{f_{A S 2}}+\frac{\rho_{F i l m} t_{F i l m}}{2 \rho_{D P O} t_{D P O}}\right) .
$$

\footnotetext{
${ }^{1}$ Dieser Effekt wird in Schwingquarz-Waagen zur in-situ Schichtdickenbestimmung ausgenutzt. Durch geeignete Wahl der Schwingungsmode wird die Schicht dabei möglichst wenig verformt, s. d. der elastische Anteil möglichst klein gehalten wird.
} 
Im amorphen Festkörper lässt sich der Schermodul als komplexe Zahl, die sich aus dem Realteil $G^{\prime}$ (Speichermodul) und dem Imaginärteil $G^{\prime \prime}$ (Verlustmodul) zusammensetzt, beschreiben:

$$
G=G^{\prime}+i G^{\prime \prime}
$$

Das Verhältnis von Verlust- zu Speichermodul ist über den inversen Qualitätsfaktor $Q^{-1}$ gegeben. In der Eulerschen Ebene wird dieser durch den Tangens des Verlustwinkels $\delta$ beschrieben. Dieser Winkel beschreibt in einer getriebenen, gedämpften Schwingung die Phasendifferenz von Antrieb zu Schwingung:

$$
Q_{\text {Film }}^{-1}=\frac{G^{\prime \prime}}{G^{\prime}}=\tan \delta
$$

Formel 4.22

Für den Betrag des Schermoduls gilt:

$$
G_{F i l m}=|G|=\sqrt{G^{\prime 2}+G^{\prime 2}}
$$

und somit für Speicher- und Verlustmodul:

$$
G^{\prime}=\frac{G_{\text {Film }}}{\sqrt{1+\left(Q_{\text {Film }}^{-1}\right)^{2}}} \text { und } G^{\prime \prime}=\frac{Q_{\text {Film }}^{-1} G_{\text {Film }}}{\sqrt{1+\left(Q_{\text {Film }}^{-1}\right)^{2}}}
$$

Zur vollständigen Beschreibung wird nun noch der Anteil der Schicht $Q_{F i l m}^{-1}$ an der der Messung zugänglichen Dämpfung des gesamten Systems $Q_{D P O+F i l m}^{-1}$ benötigt. Allgemein bezeichnet der Dämpfungsfaktor den relativen Verlust $\Delta W$ an elastischer Energie pro Schwingungsperiode, dieser setzt sich aus Anteilen von Film und Substrat zusammen:

$$
Q_{D P O+F i l m}^{-1}=\frac{1}{2 \pi} \frac{\Delta W_{D P O}+\Delta W_{F i l m}}{W_{D P O}+W_{F i l m}} .
$$

Da die Dicke der Schicht viel kleiner ist als die des DPOs, gilt mit $W_{D P O} \gg W_{F i l m}$ : $W_{D P O}+W_{F i l m} \approx W_{D P O}$ und somit: 


$$
\begin{aligned}
& Q_{D P O+F i l m}^{-1} \approx \frac{1}{2 \pi} \frac{\Delta W_{D P O}+\Delta W_{F i l m}}{W_{D P O}}=Q_{D P O}^{-1}+\frac{1}{2 \pi} \frac{\Delta W_{F i l m}}{W_{D P O}} \\
& =Q_{D P O}^{-1}+\frac{1}{2 \pi} \frac{\Delta W_{F i l m} \cdot W_{F i l m}}{W_{D P O} \cdot W_{F i l m}}=Q_{D P O}^{-1}+\frac{1}{2 \pi} \frac{\Delta W_{F i l m}}{W_{F i l m}} \frac{W_{F i l m}}{W_{D P O}} \\
& =Q_{D P O}^{-1}+Q_{F i l m}^{-1} \frac{W_{F i l m}}{W_{D P O}} \\
& \Rightarrow Q_{F i l m}^{-1} \approx\left(Q_{D P O+F i l m}^{-1}-Q_{D P O}^{-1}\right) \frac{W_{D P O}}{W_{F i l m}}
\end{aligned}
$$

Das Verhältnis der im DPO und der in der Schicht gespeicherten elastischen Energien ist im einfachen Bild einer Torsionsschwingung durch das Verhältnis der Torsionskonstanten gegeben. Mit Formel 4.18 gilt dann:

$$
\frac{W_{D P O}}{W_{F i l m}}=\frac{\kappa_{D P O}}{\kappa_{\text {Film }}}=\frac{G_{D P O} t_{D P O}}{3 G_{F i l m} t_{F i l m}} .
$$

Für den Dämpfungsfaktor der Schicht gilt somit:

$$
Q_{F i l m}^{-1} \approx\left(Q_{D P O+F i l m}^{-1}-Q_{D P O}^{-1}\right) \frac{G_{D P O} t_{D P O}}{3 G_{F i l m} t_{F i l m}} .
$$

Die Biegeschwingungen lassen sich mit denen eines Biegebalkens vergleichen, wenn man die Verformung der Flügel vernachlässigt. Die mathematische Modellierung eines Biegebalkens wird in Kapitel 4.2 beschrieben.

\subsubsection{Ex-situ Anlage}

Die ersten Experimente zur Mechanischen Spektroskopie wurden in Zusammenarbeit mit dem I. Physikalischen Institut in Göttingen durchgeführt. Details der verwendeten Anlage finden sich bei Rösner [97]. Der grobe Aufbau der Apparatur und die im Rahmen dieser Arbeit vorgenommenen Modifikationen werden im Folgenden beschrieben.

\subsubsection{Anregung und Detektion der Schwingung}

Der rückseitig mit einer dünnen Goldschicht bedampfte DPO wird in einem MiniSchraubstock am Fuß so eingespannt, dass sich die Flügel in geringem Abstand vor zwei Elektroden befinden. Die beiden so entstandenen Kondensatoren, bestehend aus jeweils einem 
Flügel und einer der Elektroden, dienen dann zur Anregung und Detektion der Schwingung. Mit einem HewletT PACKARD 3325B Frequenzgenerator wird dazu eine sinusförmige Wechselspannung an eine der Elektroden angelegt und der DPO durch elektrostatische Kräfte zum Schwingen angeregt. Zur Erhöhung der Kraft zwischen Paddelflügel und Elektrode wird der Wechselspannung an beiden Elektroden eine $200 \mathrm{~V}$ Gleichspannung überlagert, s. d. Kräfte von typischerweise $1 \cdot 10^{-7} \mathrm{~N}$ realisiert werden. Die am zweiten Kondensator durch die Relativbewegung von DPO und Elektrode erzeugte Wechselspannung wird mit einem FEMTO DLPCA-200 Current-Amplifier vorverstärkt. Die Amplitude und die Phasenlage relativ zum als Referenz verwendenden Antriebssignal werden mit einem EG\&G 5210-Lock-In Verstärker detektiert. Ein parallel geschalteter FLUKE PM 6669 Frequenzzähler misst die Frequenz der Schwingung. Der gesamte Aufbau befindet sich in einer Vakuumkammer, die mittels Vor- und Turbomolekularpumpe auf etwa $1 \cdot 10^{-7}$ mbar evakuiert werden kann, um Luftreibung, Funkenüberschläge und Kontamination zu verhindern. Die oben beschriebene Schaltung wird genutzt, um beispielsweise Frequenzscans zu durchfahren, mit deren Hilfe die unterschiedlichen Resonanzfrequenzen des Oszillators bestimmt werden können. Um die mechanischen Eigenschaften des DPOs zu bestimmen, wird er im Resonanzmodus betrieben. Das Anregungssignal wird dabei statt vom Frequenzgenerator von einer Phase-Lock-Loop (PLL) Schaltung (Princeton ApPlied Research 116 Vorverstärker, PRINCETON APPLIED ReSEARCH 124 Lock-In Verstärker mit integriertem Band-Pass-Filter) bezogen. Das Antwortsignal des Oszillators wird hier um $90^{\circ}$ phasenverschoben, verstärkt und wieder als Signal auf die Anregungselektrode gegeben. Der somit in seine Eigenmode „eingelockte“ DPO schwingt mit konstanter Amplitude in Resonanz, und seine Schwingungsfrequenz folgt der sich mit der Temperatur ändernden Resonanzfrequenz.

\subsubsection{Durchführung der Messung}

Anders als bei dielektrischen Messungen erlaubt die mechanische Spektroskopie mit resonanten Methoden nicht den direkten Zugang über einen breiten Frequenzraum, sondern nur über diskrete Resonanzfrequenzen. Stattdessen wird die Probe bei fester Frequenz, aber unterschiedlichen Temperaturen vermessen, um den Parameterraum zu erweitern. In dieser Anlage wurde im Rahmen dieser Arbeit nur auf der Antisymmetrischen Torsionsmode AS2 bei etwa $5,4 \mathrm{kHz}$ gemessen. Dazu wird der Oszillator mit einer konstanten Heiz- bzw. Kühlrate von typischerweise $0,3 \mathrm{~K} / \mathrm{min}$ temperiert. Details zum dazu benötigten Ofen finden sich im folgenden Abschnitt 4.1.2.3. Zur Messung der mechanischen Eigenschaften werden nun stetig 
Ring-Downs aufgenommen. Dazu wird die Anregung (im PLL Modus) über ein Relais abgeschaltet und der Oszillator schwingt mit exponentiell abfallender Amplitude langsam aus. Über den EG\&G Lock-In Verstärker kann mit einer typischen Zeitauflösung von $30 \mathrm{~ms}$ der zeitliche Verlauf der Amplitude $A(t)$ bestimmt werden. Zusammen mit der detektierten Frequenz $f_{0}$ kann dann über das logarithmische Dekrement $\Lambda$ die Güte $Q$ der Schwingung bestimmt werden. Es gilt:

$$
A(t)=A_{0} \exp \left(-f_{0} \cdot \pi \cdot Q^{-1} \cdot t\right) \text { mit } Q^{-1}=\frac{\Lambda}{f_{0} \cdot \pi}
$$

Die Aufnahme der Datenpunkte erfolgt dabei automatisiert über ein LABVIEW Computerprogramm, das sowohl die jeweiligen Daten der einzelnen Ring-Downs als auch die finale Messgröße $Q^{-1}$ protokolliert. Ist die Amplitude nach etwa fünf bis zehn Sekunden unter einen festen Bruchteil (typischerweise ein Drittel) der Anfangsamplitude $A_{0}$ abgefallen, wird über das Relais wieder die Anregung hinzu geschaltet und die Amplitude nimmt wieder zu. In dem unregelmäßig auftretenden Fall, dass das detektierte Signal zu schwach ist, um die Rückkopplung neu zu aktivieren, müssen Rettungsalgorithmen eingreifen, die im Rahmen dieser Arbeit entwickelt wurden. Dazu wird die Schwingung wieder extern mittels Frequenzgenerator angeregt und bei hinreichender Amplitude wird zurück in den PLL-Modus umgeschaltet. Daher ist es notwendig, während der gesamten Messung die Resonanzfrequenzen in Abhängigkeit von der Temperatur zu protokollieren. Durch lineare Extra- bzw. Interpolation ist es mit der so erhaltenen $f(T)$-Datei möglich, zu jeder momentan anliegenden Temperatur eine ungefähre Resonanzfrequenz $f_{x}$ vorherzusagen. Da, bedingt durch die hohe Güte der Schwingung des DPOs, die Resonanzfrequenz sehr scharf ist, reicht dieser interpolierte Wert allein nicht aus. Stattdessen wird ein Wert in der Nähe von $f_{x}$ angefahren und dann iterativ die Frequenz erhöht, bis die Amplitude einen Wert erreicht, der ein erneutes Umschalten in den PLL-Modus erlaubt.

\subsubsection{Temperaturregelung}

Zur Paddelheizung werden die Glühwendeln zweier Projektorlampen als Strahlungsofen verwendet, die Temperaturkontrolle erfolgt durch ein im Schraubstock angebrachtes Thermoelement, die Regelung übernimmt ein EUROTHERM 3508 PID Regler. Da eine in-situ Tempera- 
turbestimmung am schwingenden Oszillator nicht möglich ist ${ }^{1}$, sind Kalibriermessungen notwendig, die eine Relation zwischen der jederzeit zugänglichen Fußtemperatur und der physikalisch relevanten Kopftemperatur des DPOs erlaubt. Dazu wird mit Leitsilber ein kleines $\mathrm{NiCr} / \mathrm{Ni}$-Thermolelement an den Kopf eines Referenzoszillators geklebt und es werden mehrere Heiz- und Kühl-Zyklen mit unterschiedlichen Heizraten durchgefahren.

Abbildung 4.5 zeigt die Ergebnisse dieser Messungen für Heizraten von 0,6 bzw. 1,2 K/min. Der Vergleich von Soll- und Ist-Temperatur des Halters zeigt für beide Heizraten die sehr gute Regelcharakteristik der Temperaturregelung. Beim Heizen, Halten und Kühlen von Temperaturen über $350 \mathrm{~K}$ liegen die roten und schwarzen Kurven gut übereinander. Erst wenn die Temperaturdifferenz zur Raumtemperatur zu gering wird, weicht die Kurve der tatsächlichen Temperatur von der vorgegebenen Temperatur ab und die Regelung versagt, da die Probe nicht schnell genug abkühlen kann. Der Punkt des Abweichens kann durch die Wahl einer geringeren Kühlrate zu tieferen Temperaturen verschoben werden. Im Vergleich von Halterund Kopftemperatur wird der Schwachpunkt der Methode deutlich. Besonders bei den Halteund Wendepunkten in der Temperatur fallen unregelmäßige Abweichungen zwischen Fußund Kopf-Temperatur auf.

Auch diese Abweichungen sind bei kleinerer Heizrate schwächer ausgeprägt. Daher wurden alle weiteren Messungen mit einer Heizrate von nur 0,3 K/min durchgeführt. Abbildung 4.6 (links) zeigt das zugehörige Temperaturprofil mit minimalen Variationen zwischen Soll- und Ist-Temperatur des Halters und nur schwachen Überschieß-Effekten der DPO-Temperatur an den Temperatur-Halte- und Wendepunkten. In den Bereichen konstanter Heizrate lässt sich ein parabolischer Zusammenhang zwischen Fuß- und Paddeltemperatur anpassen. Die erhaltenen Funktionen sind in Abbildung 4.6 (rechts) angegeben. Diese dienen im Weiteren zur Umrechnung zwischen Fuß- und DPO-Temperatur.

Bereits Rösner sah den limitierenden Faktor der Messgenauigkeit des Doppelpaddeloszillators in der Temperaturmessung [97]. Dieser Eindruck kann hier bestätigt werden. Ursache dafür ist zum einen eine mögliche inhomogene Temperaturverteilung am Paddel. Zum anderen ist die Temperaturbestimmung über die Kalibriermessung fehlerbehaftet, da bereits das Anbringen eines Thermoelements die Wärmeableitung vom DPO stark verändern kann und damit die Messung verfälscht. Außerdem ändert eine Beschichtung des DPOs dessen Absorptionsgrad

\footnotetext{
${ }^{1}$ Kontaktmessungen scheiden aus, da sie die freie Schwingung des Oszillators verhindern. Pyrometrische Messungen scheitern an der hohen Transparenz des Siliziums im Infraroten Spektrum.
} 
und somit die effektive Wärmeaufnahme der Strahlungsheizung. Während der relative Fehler der Temperatur bei Prozessführung mit konstanter Heizrate mit maximal $\pm 3 \mathrm{~K}$ abgeschätzt werden kann, ist der absolute Fehler mit der hier verwendeten Strahlungsheizung sicherlich um einen Faktor fünf größer.
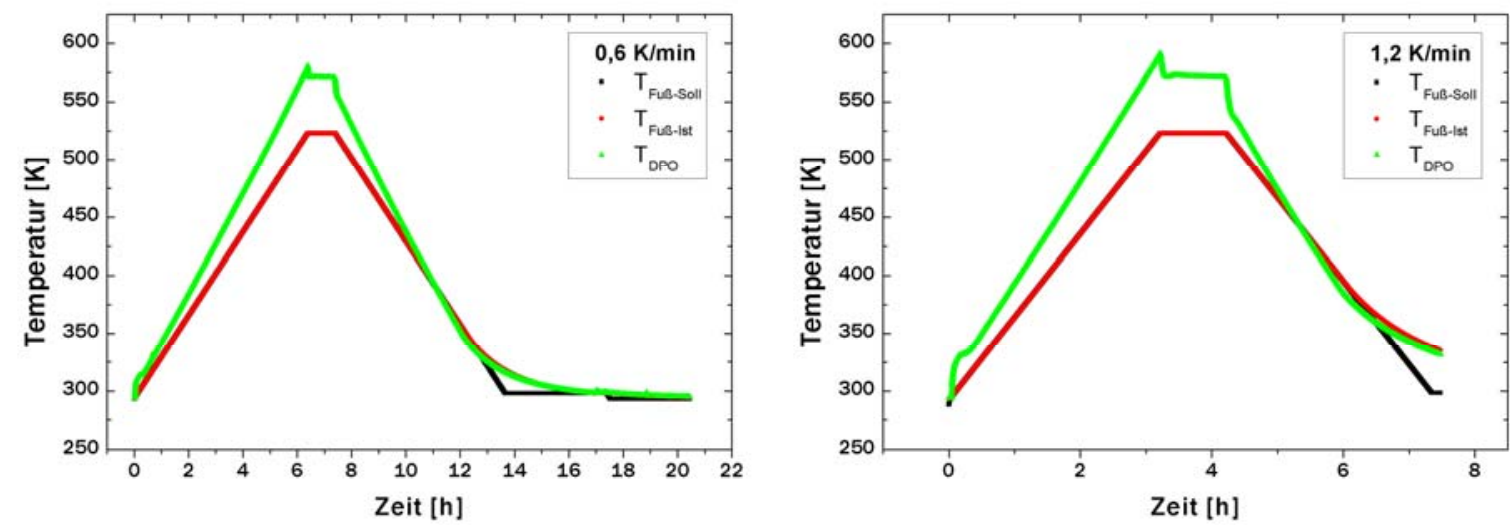

Abbildung 4.5: Temperaturprofile beim Durchlaufen eines Heiz- und Kühlzyklus mit Heizraten von 0,6 K/min (links) und 1,2 K/min (rechts). Soll- und Ist-Temperatur des Halters liegen bei beiden Heizraten im mittleren Temperaturbereich gut übereinander, erst bei Kühlung unter ca. $350 \mathrm{~K}$ reicht die Abkühlung des Halters nicht mehr aus, um der Soll-Temperatur zu folgen. Die Temperatur des DPOs reagiert stark auf Änderung der Heizrate, sichtbar im Einschwingverhalten am Anfang der Messung und beim Umschalten von Heizen auf Halten, bzw. Halten auf Kühlen.
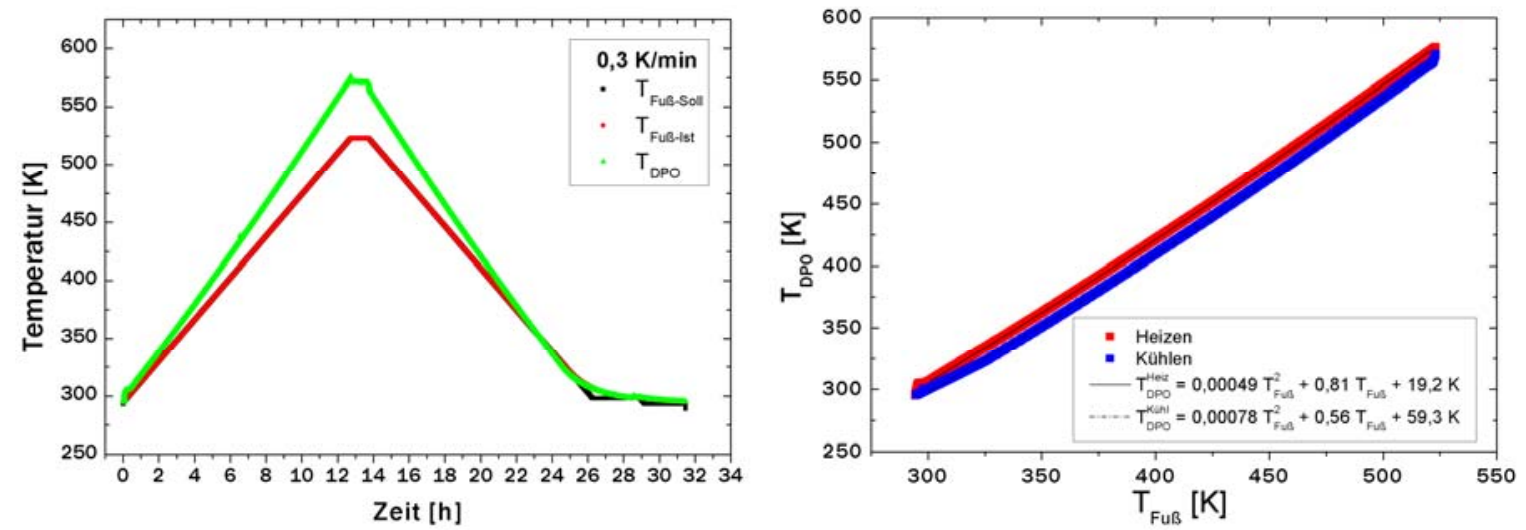

Abbildung 4.6: Links: Temperaturprofil beim Durchlaufen eines Heiz- und Kühlzyklus mit einer geringen Heizrate von $0,3 \mathrm{~K} / \mathrm{min}$ zeigt über den gesamten Temperaturbereich nur minimale Abweichung zwischen Soll- und IstTemperatur des Halters. Rechts: Für konstante Heizraten lässt sich eine parabolische Abhängigkeit der DPOTemperatur von der Fußtemperatur anpassen.

\subsubsection{In-situ Anlage}

Für weitere Experimente wurde eine neue Apparatur entwickelt und in die in der Arbeitsgruppe um Prof. Krebs vorhandene PLD-Beschichtungsanlage integriert. Diese bringt zunächst den Vorteil der möglichen in-situ Messung von Polymeren mit sich, da in der Anlage 
des I. Physikalischen Instituts durch Elektronenstrahlverdampfung nur metallische Systeme deponiert werden können. Weiterhin wurden die Konzepte der Oszillator-Heizung und der mechanischen Anregung neu überdacht und neue Lösungen konzipiert: Bei der Heizung wurde die Strahlungsheizung durch einen Ofen ersetzt, der die DPOs über eine Kombination aus Wärmeleitung und -strahlung temperiert. Durch den Einbau eines $\mathrm{LN}_{2}$-Kühlfingers konnte der Temperaturbereich nach unten auf bis etwa $200 \mathrm{~K}$ erweitert werden. Insgesamt konnte bei den hier verwendeten vergleichsweise niedrigen Temperaturen bis maximal $675 \mathrm{~K}$ eine höhere Temperaturstabilität und bessere Reproduzierbarkeit erreicht werden.

Die Anregung erfolgt nicht über elektrostatische Kräfte, sondern extern mechanisch. Dazu wird ein handelsüblicher Breitbandlautsprecher verwendet, der den Vakuumflansch, an dem der DPO angebracht ist, zum Schwingen anregt, wodurch bei geeigneter Frequenz auch der DPO selbst zum Schwingen gebracht wird. Die Messung der Schwingung erfolgt über eine Kombination aus Lichtzeiger und positionssensitivem Detektor. Dadurch können zwei Problemquellen, die bei der elektrostatischen Anregung auftauchen, umgangen werden: Zum einen sind Anregung und Detektion strikt getrennt. Der Einfluss elektrischer Störsignale, wie etwa das „Übersprechen“, also ein Signalübertrag von der anregenden zur detektierenden Elektrode, der bei zu großen Anregungsspannungen auftreten kann, ist somit ausgeschlossen. Bei der elektrostatischen Anregung besteht zum anderen die Gefahr, die Elektroden mit deponiertem Material zu kontaminieren und ihre elektronischen Eigenschaften zu verändern. Solche Störeffekte treten bei der externen Anregung ebenfalls nicht auf.

\subsubsection{Aufbau}

Als Träger für den gesamten Aufbau innerhalb der PLD-Beschichtungsanlage dient ein über ein Gewinde längenverstellbares Edelstahlrohr, das an einem DN40 Kammerflansch mit Sichtfenster angebracht ist (Abbildung 4.7). Auf diesem Edelstahlrohr ist mit drei auf Spiralfedern gelagerten Schrauben ein $\mathrm{Cu}$-Becher angebracht, der von mehreren Wicklungen eines Mantelheizleiters umfasst wird. Der komplette heizbare Aufsatz ist somit in allen drei Kipprichtungen justierbar und, da der einzige Wärmekontakt über die drei dünnen Edelstahlschrauben gegeben ist, thermisch gut von der Vakuumkammer isoliert. Auf dem Kupferbecher ist eine Kupfer-Abschlussplatte angebracht, auf die wiederum ein Mini-Schraubstock aus Edelstahl, der den DPO aufnimmt, zusammen mit einem massiven Kupferrahmen aufgeschraubt ist. Mit diesem Aufbau lässt sich der DPO hervorragend im Temperaturbereich zwischen Raumtemperatur und $675 \mathrm{~K}$ temperieren. Zur Erweiterung des Messbereichs unterhalb 
von Raumtemperatur kann optional noch ein kupfernes Kältereservoir über die Mantelheizleitungen geklemmt werden. Über ein Band aus Kupfergeflecht wird dieses dann mit einem an einem zweiten Flansch angebrachten und von flüssigem Stickstoff durchflossenen Kupferblock verbunden, s. d. am DPO Temperaturen von unter $200 \mathrm{~K}$ zu realisieren sind.

Abbildung 4.8 zeigt links den Heizbecher mit aufgesetztem Kältereservoir und den noch nicht montierten DPO-Halter mit Kupferrahmen. Im rechten Teil des Bildes ist der Schraubstock mit Kupferrahmen vor der Kupferplatte montiert. Halbtransparent eingezeichnet ist die Position eines im Schraubstock eingespannten DPOs. Die Pfeile markieren die kleine Bohrung, die das zur Temperaturkontrolle verwendete Mantelthermoelement aufnimmt. In der Kupferplatte ist die Blende zu erkennen, die der Strahl des außerhalb der Vakuumkammer montieren Lasers, der die Schwingung des DPOs abtastet, passieren kann.

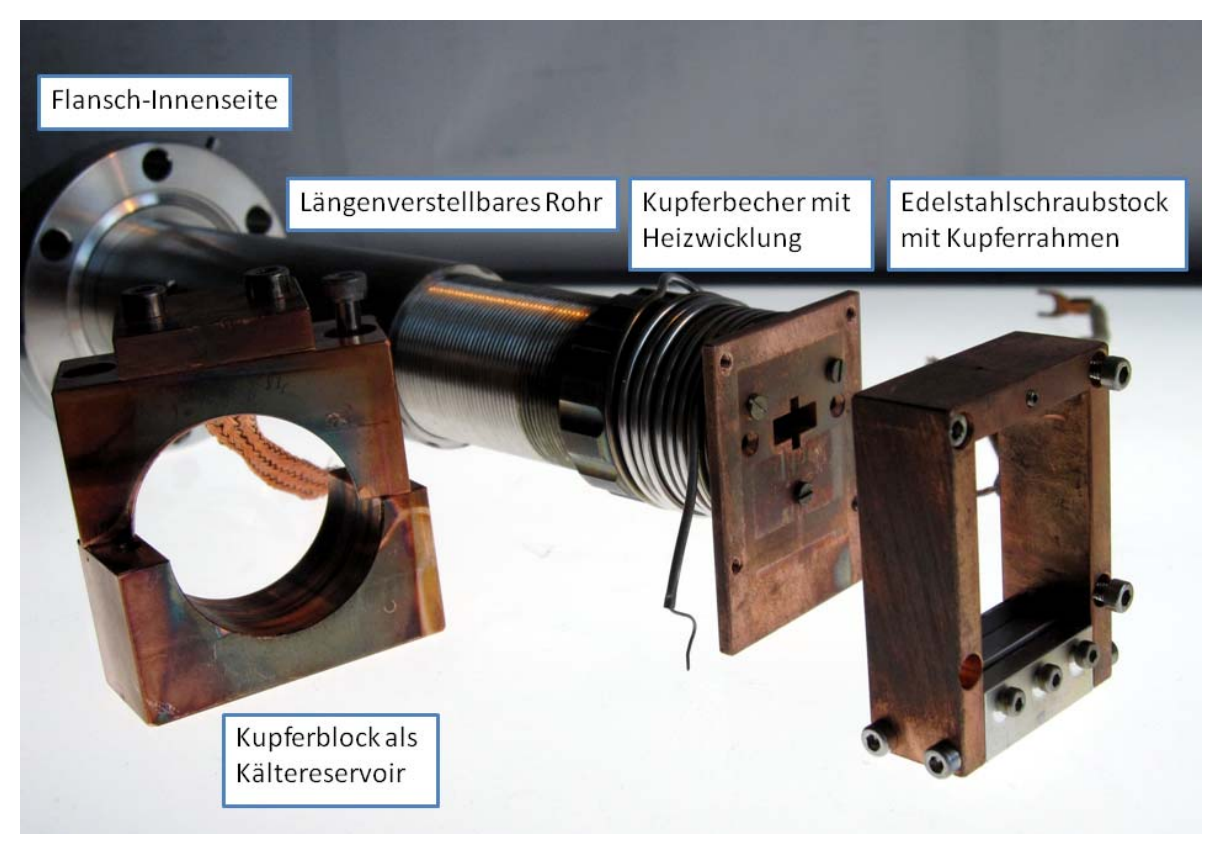

Abbildung 4.7: Komponenten der Doppelpaddelhalterung mit Heiz- und Kühlsystem. 


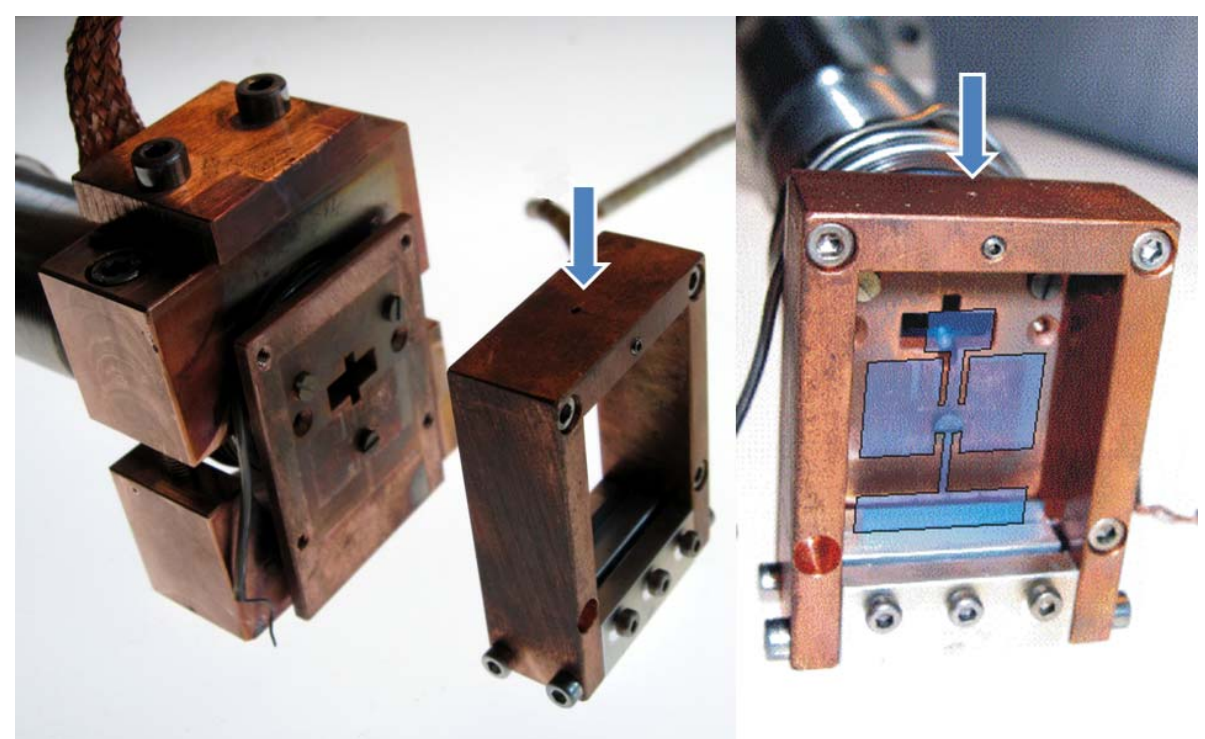

Abbildung 4.8: Detailaufnahmen der Oszillator-Halterung. Links: Becherförmiger Ofen mit Mantelheizelement und aufgesetztem Kühlreservoir. Über die drei Schrauben an der Kupferplatte ist der Halter in alle drei Kipprichtungen justierbar. Rechts: Detailaufnahme des Mini-Schraubstocks mit stilisiertem DPO. Der Pfeil markiert in beiden Bildern die Bohrung für das Mantelthermoelement.

\subsubsection{Mechanische Anregung und Messwerterfassung}

Außerhalb der Kammer ist der Vakuumflansch über eine Adapterplatte fest mit einem Breitbandlautsprecher verbunden, s. d. die Schwingungen der Lautsprechermembran direkt zur DPO-Halterung übertragen werden. Als Signal des Lautsprechers dient das mit einem DETON D75W verstärkte Sinussignal eines AGILENT 33200A Frequenzgenerators.

Abbildung 4.9 zeigt den schematischen zur Messwerterfassung notwendigen Aufbau. Dieser liegt außerhalb der Vakuumkammer räumlich isoliert von dieser vor. Der Strahl eines Diodenlasers trifft durch das oben beschriebene Flanschfenster und die Blende in der Cu-Platte auf den rückseitigen Kopf des DPOs. Der reflektierte Strahl passiert Blende und Fenster erneut und trifft dann auf einen justierbaren zweidimensionalen positionsempfindlichen Detektor. 


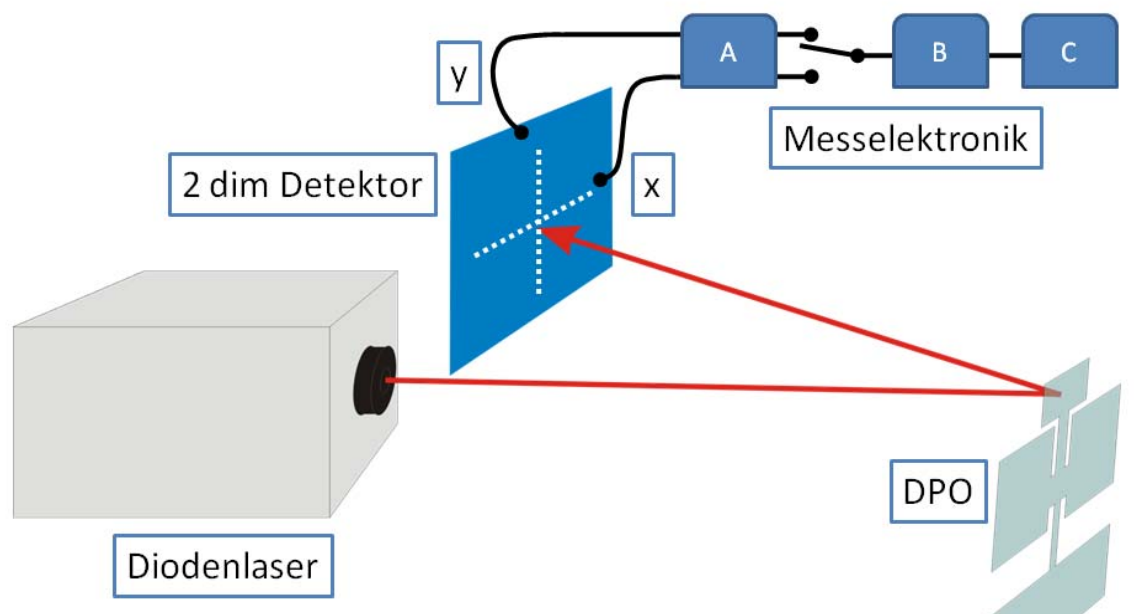

Abbildung 4.9: Aufbau zur Detektion der DPO-Schwingung. Die Position des vom DPO-Kopf reflektierten Strahls eines Diodenlasers wird mit einem 2-dimensionalen Detektor registriert und von der Elektronik A in ein Spannungssignal umgewandelt und vorverstärkt. Je nach Schwingungsart (Biegung oder Torsion) wird das x- oder y-Signal über einen Umschalter auf einen Lock-In Verstärker B gegeben und gefiltert bevor es über AD-DA-Wandlerkarte C zur rechnergestützten Erfassung weitergeleitet wird.

Die Position des Strahls auf dem Detektor wird mit hoher räumlicher Auflösung und geringer Reaktionszeit in zwei Stromsignale, eines für die x- und eines für die y-Position, umgewandelt. Diese Signale werden in Elektronik A in Spannungssignale umgewandelt und vorverstärkt. Prinzipiell ist mit diesem Aufbau die gleichzeitige Messung von Torsions- und Biegeschwingungen möglich, da beide Achsen unabhängig voneinander ausgelesen werden können. Im Rahmen dieser Arbeit werden allerdings stets monofrequente Schwingungen untersucht, da interne Schwingungsmoden bei diskreter Frequenz abgefragt werden sollen. Durch die mit der Superposition verschiedener externer Frequenzen verbundene Mischung der Anregung interner Prozesse wären hier klare Trennungen unmöglich. Daher wird der DPO jeweils nur in einer Resonanzmode betrieben. Je nach Art der Schwingung ist dann klar zwischen einem Lichtzeigerausschlag in x-Richtung für eine Torsionsschwingung und in y-Richtung für eine Biegeschwingung zu unterscheiden (Vgl. Abbildung 4.3). Die jeweils resultierende Wechselspannung wird durch ein Relais ausgewählt und mit einem ITHACO 3961B Lock-In Verstärker (in Abbildung 4.9 mit B gekennzeichnet) Bandpass-gefiltert und verstärkt. Als Referenzsignal dient dabei die Anregungsspannung des Frequenzgenerators. Schließlich wird das Signal in den Line-in Eingang einer AD-DA-Wandlerkarte (CREATIVE SoundBLASTER Audigy4) mit einer hohen Samplingrate von $192 \mathrm{kHz}$ zur rechnergestützten Erfassung weitergeleitet (in Abbildung 4.9 mit C gekennzeichnet). 


\subsubsection{Temperaturregelung}

Zur Heizung des Kupferbechers und somit des kompletten vorderen Aufsatzes wird die von der Apparatur zur dielektrischen Messung bekannte Kombination aus EUROTHERM 3508 PID Regler und KNÜRR-HeINZINGER POLARIS 35-20 Netzteil verwendet (Vgl. Kapitel 3.7). Dabei werden je nach Aufbau, mit und ohne Kältereservoir, angepasste Regelparameter verwendet.

Wie bereits in früheren Arbeiten festgestellt wurde [97], ist die gleichmäßige Temperierung des DPOs eine technische Herausforderung. Eine Heizung allein über Wärmeleitung ist nicht möglich, da über den kleinen Querschnitt des Oszillator-Fußes, zusammen mit der geringen Wärmeleitfähigkeit des Siliziums nicht genügend Wärme zum oberen Teil des DPOs fließen kann, da über die großen Paddelflächen bereits bei niedrigen Temperaturen viel Energie über Wärmeabstrahlung verloren geht. Bei der Heizung rein über Wärmestrahlung stellt sich das Problem der schlechten Kontrollmöglichkeit der momentanen Temperatur und einer wenig homogenen Temperaturverteilung (Vgl. Kapitel 4.1.2.3). In der hier verwendeten Anlage wurde daher auf eine Kombination von Wärmeleitung und -strahlung zurückgegriffen. Zum einen wird dem DPO über den Schraubstock Wärme über Wärmeleitung zugeführt. Zum anderen dient der Rahmen aus Kupfer, der um den DPO herum angeordnet ist, zur Temperierung über Strahlungsheizung.

Da die direkte Messung der absoluten Temperatur des DPOs schwierig ist, weil das Anbringen auch nur eines kleinen Wärmeleiters wie eines Thermoelementes die Temperatur des filigranen DPOs sofort beeinflusst, wird hier der Zugang über Simulationen mit der FinitenElemente-Methode vorgestellt. Statt der Modellierung des kompletten Heizbechers geht in der Simulation der Wärmestrom gleichmäßig von der hinteren Oberfläche der Kupferplatte aus. Als mathematische Randbedingung ist die Temperatur hier für alle Zeiten auf eine feste Temperatur $T_{\text {Platte }}$ festgesetzt. Somit wird die komplette Regelung der Heizung vernachlässigt. Bei hinreichend guter Wärmeleitfähigkeit (Kupfer) und gut gewählten PID Parametern ist diese Näherung zulässig, da die Steuerung auf einen ausreichend gut kontaktierten Temperaturbezugspunkt regeln kann und die gewünschten Temperaturen an diesem Messpunkt und somit im kompletten kupfernen Bereich gut eingestellt werden können.

Abbildung 4.10 zeigt den simulierten Temperaturverlauf an Ofen und DPO bei Einstellung eines sehr großen Temperaturschrittes von $100 \mathrm{~K}$. Dabei wurde bei $t=0$ die Temperatur von Ofen und DPO auf $300 \mathrm{~K}$ gesetzt, $T_{\text {Platte }}$ beträgt $400 \mathrm{~K}$. Neben Wärmeleitung ist in der Simu- 
lation auch Wärmestrahlung über ein sogenanntes „Surface2Surface-Modell“ einbezogen. Der Oszillator und die Innenseiten des Ofens tauschen also über Wärmestrahlung Energie aus. Bedingt durch die gute Wärmeleitfähigkeit des Kupfers egalisiert sich die Temperatur des Kupfers bereits nach wenigen Sekunden. Nach etwa 25 Sekunden ist auch der Edelstahlhalter nahe der Gleichgewichtstemperatur. Auch der DPO ist trotz des großen Temperaturschritts schon nach etwa fünf Minuten im Gleichgewicht.

Der Inset in Abbildung 4.10 zeigt den stationären Zustand nach einer Simulationszeit von acht Minuten (Man beachte die abweichende Skala im Inset). Hier bleibt ein Temperaturgradient von etwa $5 \mathrm{~K}$ zwischen Fuß und Kopf, am größten ist der Effekt aufgrund des geringen Querschnitts am Bein des Oszillators. Dieser Temperaturunterschied wächst mit steigender Temperatur weiter an. Simulationen des Heizens von 490 auf $500 \mathrm{~K}$ zeigen, dass im Gleichgewicht noch etwa $15 \mathrm{~K}$ Unterschied zwischen Paddelfuß und Paddelkopf anliegen.
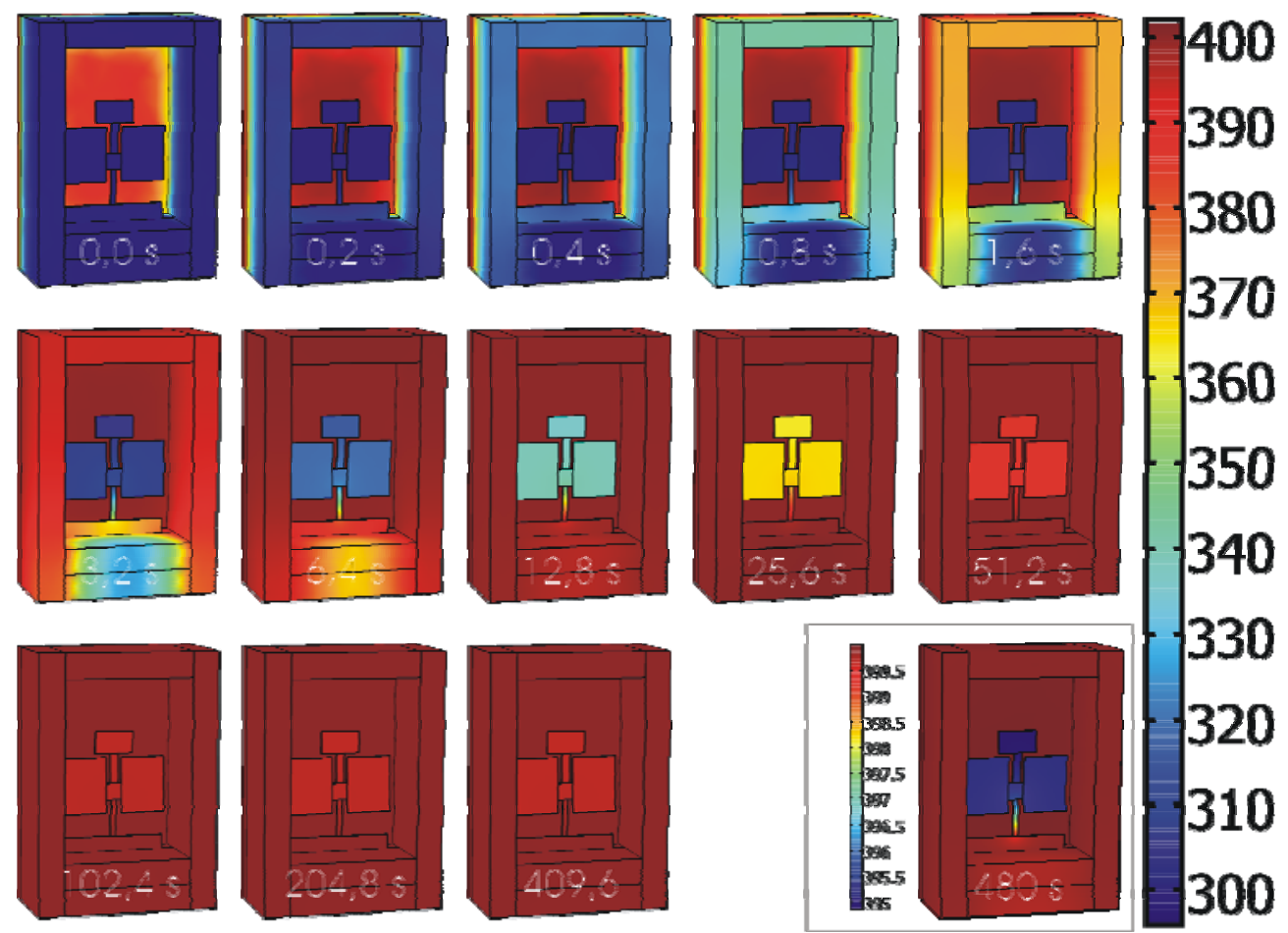

Abbildung 4.10: Mittels FEM bestimmte Temperaturentwicklung an Ofen und DPO während des Aufheizens. Bei $t=0$ beträgt die Temperatur von Ofen und DPO $300 \mathrm{~K}$. Die Rückwand des Ofens hat bei allen Zeiten $T_{\text {Platte }}=400 \mathrm{~K}$. Im Inset ist der stationäre Zustand nach acht Minuten zu sehen. Der Temperaturunterschied zwischen Oszillator-Kopf und Halter beträgt etwa $5 \mathrm{~K}$.

Während die Temperatur am DPO zeitlich also sehr stabil ist, ist die absolute Temperatur des DPOs mit einem mit der Temperatur wachsenden Fehler behaftet. Im Bereich der hier untersuchten Temperaturen ist dieser Fehler hinzunehmen. Sollten in weiteren Untersuchungen höhere Temperaturen von Interesse sein, könnte ein beweglicher Hitzeschild, der den Ofen 
nach vorne abschließt und dabei idealerweise in thermischen Kontakt zum Kupferrahmen steht, große Vorteile bringen.

\subsubsection{Messverfahren}

Die komplette Messung ist mittels eines im Rahmen dieser Arbeit entwickelten LABVIEW Programms automatisiert und gliedert sich in vier Teile: Temperatur einstellen, Resonanzfrequenz suchen, Ring-Down durchführen und Auswertung. Abbildung 4.11 zeigt den schematischen Ablauf der Messung für eine Temperaturstufe. Zunächst wird der DPO auf die gewünschte Temperatur gebracht. Die Auswahl der Temperatur erfolgt über eine Tabelle, die für mehrere Temperaturprofile Start-, und End-Temperatur, Temperaturschrittweite, Heizrate und Wartezeit beinhaltet. Nach Erreichen der Soll-Temperatur am Ofen wird noch typischerweise 10 Minuten gewartet, bis auch der DPO im thermischen Gleichgewicht ist.

- Temperatur anfahren

- Warten

$\rightarrow$ Temperatur stabil

- Frequenz suchen

- $f(T)$-Datei lesen, lineare Regression

$\rightarrow$ Schätzfrequenz $f\left(T_{0}\right)$

- Frequenzscan um $T_{0}$

- Lorentz-Anpassung

$\rightarrow$ Peaklage $f_{\max }$ und Halbwertsbreite $\omega$

- Anregungsfrequenz $f_{\text {Anr }}(T)=f_{\max }-\omega^{*} C$

- Anregen und Ringdown

- Amplitudencheck

- Anregen, Einschwingen

- Ringdown $5 x$

$\rightarrow$ Amplitudenverlauf $A(t)$ und Frequenz $f_{0}$

- Auswertung und Schreiben der Daten

$\rightarrow A(t), f_{0}, Q^{-1}, f_{A n,}, U_{\text {Anr }}$ Ampl.-Res.-Funktion, Lorentzfit

Abbildung 4.11: Programmablauf der Messung von Resonanzfrequenz und Dämpfung für eine Temperatur.

Die folgenden drei Schritte werden nacheinander für alle zu messenden Moden durchgeführt. Als Datengrundlage dienen wiederum zwei Tabellen, die die wichtigsten Kenndaten für die Moden beinhalten.

Zum Bestimmen der jeweiligen Resonanzfrequenz steht zusätzlich für jede Mode eine $f(T)$-Datei bereit, in der die Resonanzfrequenzen für unterschiedliche Temperaturen protokol- 
liert werden. Mit Hilfe einer linearen Regression an diese Daten kann dann bei der Temperatur $T_{0}$ eine Schätzfrequenz $f\left(T_{0}\right)$ bestimmt werden. Die Amplitudenresonanzfunktion um diese Schätzfrequenz wird mittels eines Frequenzscans ermittelt und mittels einer Lorentzfunktion angepasst. Um jederzeit im anregenden Bereich, also unterhalb der Resonanzfrequenz zu bleiben, wird von der so ermittelten Maximumsfrequenz $f_{\max }$ ein Bruchteil $x$ der Halbwertsbreite $\omega$ der Lorentzfunktion abgezogen und so die Anregungsfrequenz $f_{\text {Anr }}=f_{\max }-x \omega$ bestimmt. Mit dieser Frequenz wird über den Frequenzgenerator der Lautsprecher angesteuert. Durch Anpassung des Ausgangspegels $U_{\text {Anr }}$ wird die Schwingungsamplitude des DPOs bei Bedarf iterativ angepasst, bis sie in einem vordefinierten Fenster liegt.

Nach kurzer Einschwingzeit können dann Ring-Downs aufgenommen werden. Dazu wird das Anregungssignal komplett abgeschaltet und nach kurzer Einschwingphase wird die Amplitude $A(t)$ der Schwingung mit einer zeitlichen Auflösung von $150 \mathrm{~ms}$ aufgenommen, bis sie einen Schwellwert von einem Drittel der Anfangsamplitude unterschreitet. Aus $A(t)$ können die Frequenz der freien Schwingung und das logarithmische Dekrement jedes einzelnen Ring-Downs bestimmt werden. Danach wird die Anregung wieder eingeschaltet und die gesamte Messung zur Verbesserung der Statistik typischerweise insgesamt fünfmal durchgeführt.

Nach der letzten Einzelmessung werden die Ergebnisse einem Ausreißertest unterzogen und zu einem Mittelwert zusammengefasst. Zur Kontrolle während der Messung wird bereits hier der inverse Qualitätsfaktor $Q^{-1}$ bestimmt. Bevor der Vorgang bei der nächsten Schwingungsmode wiederholt wird, werden alle Rohdaten der Einzelmessungen sowie die gemittelten Daten gespeichert. Sind alle gewünschten Frequenzen abgearbeitet, wird der komplette Ablauf bei der nächsten Temperatur wiederholt.

\subsubsection{Auswertung der Messung}

Unabhängig von den Spezifikationen der beiden Messanordnungen, also der Art der Anregung und der unterschiedlichen Ofengeometrien, zielt die Auswertung der verschiedenen Messergebnisse auf die Bestimmung der elastischen Eigenschaften der auf den DPO aufgebrachten dünnen Schichten ab. Grundlage dafür sind bei beiden Anlagen die temperaturabhängigen Daten der Resonanzfrequenz $f_{0}$ und des inversen Qualitätsfaktors $Q^{-1}$, die bereits vom Messprogramm berechnet werden. Zusätzlich wird noch die Schichtdicke $t$ benötigt, die unter der legitimen Annahme einer konstanten Depositionsrate über das Vermessen von Referenzproben bestimmt werden kann. Zur qualitativen Analyse werden im Rahmen dieser Arbeit nur 
die Ergebnisse der AS2 Mode verwendet, da diese zum einen die geringste Eigendämpfung und somit eine besonders hohe Empfindlichkeit besitzt. Zum anderen ist hier nahezu ausschließlich die Verformung des Halses an der Schwingung beteiligt. Es liegt also eine reine Torsionsbewegung vor, s.d. die Auswertung der Ergebnisse Rückschlüsse auf das reine Schermodul der Schicht erlaubt. Außerdem ist die Temperaturverteilung oberhalb des DPOFußes sehr homogen, wie in Kapitel 4.1.3.3 gezeigt wurde. Temperaturgradienten, die z. B. bei den Biegemoden die Messung leicht beeinflussen können, sind hier auszuschließen.

\subsection{Das Plasma Plume eXcited Reed (PPXR)}

Neben dem hochsensiblen Doppelpaddeloszillator wird im Rahmen dieser Arbeit auch eine Variation der klassischen Biegebalkenmethode „vibrating reed“"verwendet [98]. Das Plasma Plume eXcited Reed, PPXR wurde hier in Göttingen von Scharf entwickelt [99].

\subsubsection{Aufbau, Anregung und Detektion}

Kernstück des PPXRs ist ein $100 \mu \mathrm{m}$ dicker Siliziumbalken, der als schwingendes Substrat für die zu untersuchende Schicht dient.

Abbildung 4.12 zeigt den schematischen Aufbau der Anlage. Der in einer Vakuumanlage montierte Biegebalken kann ausgelenkt und so zum freien Schwingen angeregt werden, indem er in der PLD-Anlage mit einem Silber-Puls beschichtet wird. Der Impuls der Teilchen in der Plasmakeule, die auf den Balken treffen, bewirkt eine Auslenkung des Balkens, s. d. dieser dann größtenteils mit seiner ersten Eigenmode ausschwingt. Durch geeignete Wahl der Geometrie des Biegebalkens lassen sich Resonanzfrequenzen zwischen $500 \mathrm{~Hz}$ und $10 \mathrm{kHz}$ realisieren. Die Schwingung wird über einen Lichtzeiger in Verbindung mit einem positionsempfindlichen Detektor detektiert und über eine AD-DA Wandlerkarte an einen Computer übergeben. Um temperaturabhängige Messungen durchführen zu können, ist die Halterung des Biegebalkens an einen Ofen bzw. einen Kühlfinger gekoppelt, s. d. in Verbindung mit einem KNÜRR-HEINZINGER PolarIS 35-20 Netzteil mit einem EUROTHERM 3508 PID Regler Temperaturen zwischen 200 und $600 \mathrm{~K}$ realisierbar sind. 


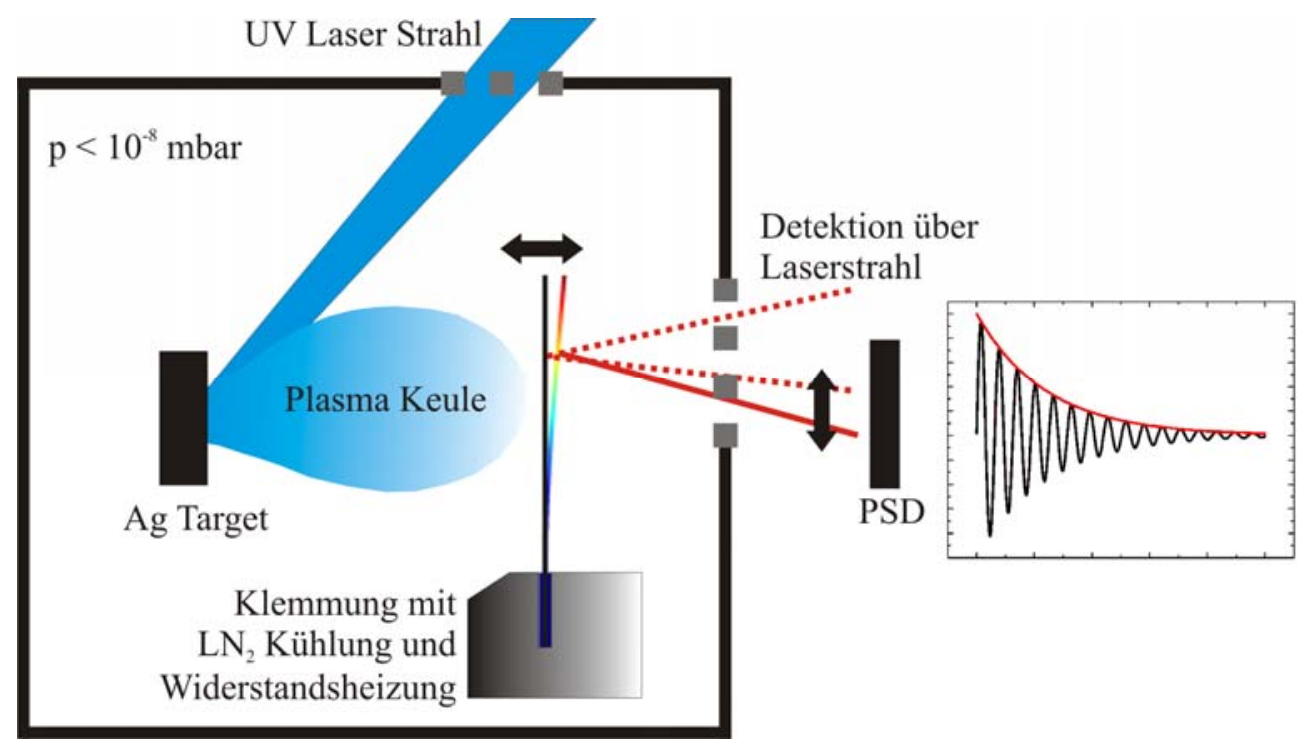

Abbildung 4.12: Schematischer Aufbau der PPXR Apparatur. Der Impuls der Ag-Teilchen in der Plasmakeule, die auf den Biegebalken treffen, lenken diesen aus, woraufhin er frei ausschwingt. Über einen Lichtzeiger kann die Auslenkung detektiert werden.

Im Vergleich zur Methode des Doppelpaddeloszillators zeigen sich zwei Unterschiede auf: Erstens ist die Güte der Schwingung aufgrund der nicht optimierten Geometrie etwa eine Größenordnung schlechter als die der AS2 Mode des DPOs. Begrenzender Faktor ist hier die Energiedissipation über die Klemmung. Zweitens ist die Form der Schwingung beim PPXR aufgrund der einmaligen Auslenkung, gefolgt von freier Schwingung auf die erste Biegemode beschränkt. Um höhere Moden vermessen zu können, wäre hier eine kompliziertere externe Anregung notwendig, die dem schlanken Aufbauprinzip dieser Methode widersprechen würde. Die in-situ-Messung bei unterschiedlichen Frequenzen bleibt also dem DPO vorbehalten.

\subsubsection{Durchführung und Auswertung}

Die Aufnahme der Messpunkte erfolgt auch bei diesem Verfahren mit einem rampenförmigen Temperaturprofil. Zunächst wird die nächste Temperaturstufe angefahren, dann wird fünf Minuten gewartet, bis das System sicher im thermischen Gleichgewicht ist. Die Messwertaufnahme an der Wandlerkarte wird gestartet und zur Anregung wird ein Laserpuls abgegeben. Nach einer definierten Zeit zwischen 2 und 10 Sekunden wird die Aufnahme an der Wandlerkarte gestoppt. Zur Verbesserung der Statistik werden pro Temperaturschritt typischerweise drei Messungen durchgeführt. Die im wav-Format gespeicherten Messdaten werden zunächst grob Fourier-gefiltert (Bandsperrfilter über die $50 \mathrm{~Hz}$ des Stromnetzes und die $1.000 \mathrm{~Hz}$ der Turbomolekularpumpen) und dann weiter ausgewertet. Dazu wird ausgehend vom absoluten 
Maximalwert der Messdaten an jede Halbschwingung (begrenzt durch die jeweiligen Messwerte mit entgegengesetztem Vorzeichen) eine Sinus-Schwingung $A_{0} \sin (\omega t+\varphi)$ angepasst, s. d. für jede Halbschwingung die Lage und die Amplitude des Extremwerts bestimmt werden kann. Die somit erhaltenen Parameter des gesamten Schwingungsverlaufs werden einem Ausreißertest unterzogen und man erhält das logarithmische Dekrement und die Resonanzfrequenz, also die zur Bestimmung des inversen Qualitätsfaktors $Q^{-1}$ benötigten Werte für jeden Ring-Down.

Zur Bestimmung des komplexen Elastizitätsmoduls ist auch hier, ähnlich wie in Kapitel 4.1.4, etwas klassische Mechanik notwendig. Der Realteil des Elastizitätsmoduls eines einseitig eingespannten Balkens der Dicke $t$ und der Breite $b$ ergibt sich nach [100] zu:

$$
E^{\prime}=12 \frac{k}{b t^{3}}
$$

Dabei bezeichnet $k$ die Biegesteifigkeit, also die gespeicherte Energie pro Länge und Krümmung, die von der als temperaturunabhängig angenommenen Massendichte $\rho$ (Masse pro Länge), der temperaturabhängigen Resonanzfrequenz $\omega=2 \pi f_{0}$ und dem temperaturunabhängigen Parameter $\gamma$ abhängt:

$$
k(T)=\frac{\rho}{\gamma^{4}} \omega^{2}(T)
$$

$\gamma$ lässt sich abhängig von der Länge des Balkens $l$ durch Lösung des Differentialgleichungssystems eines gedämpft schwingenden Balkens bestimmen. Für die Grundmode gilt: $\gamma=1,8751 / l$. Formel 4.30 in Formel 4.31 eingesetzt liefert dann den temperaturabhängigen Speichermodul des Substrats.

Der Speichermodul eines zusätzlichen dünnen Films ergibt sich durch Integration über dessen Biegesteifigkeit von der Unterkante des Substrates bis zur Oberkante des Films zu:

$$
\begin{aligned}
& E_{\text {Film }}^{\prime}=\frac{k_{\text {Film }}}{b\left(\frac{1}{4} t_{\text {Substrat }}^{2} t_{\text {Film }}+\frac{1}{2} t_{\text {Substrat }} t_{\text {Film }}^{2}+\frac{1}{3} t_{\text {Film }}^{3}\right)} \\
& \approx 4 \frac{k_{\text {Film }}}{b t_{\text {Substrat }}^{2}}=4 \frac{k_{\text {Substrat }+ \text { Film }}-k_{\text {Substrat }}}{b t_{\text {Substrat }}^{2} t_{\text {Film }}}
\end{aligned}
$$


wobei die Näherung zulässig ist, weil die Dicke des Films sehr viel kleiner ist als die des Substrats. Die Dicke des polymeren Films wird dabei mit der Annahme bestimmt, dass für Temperaturen weit über der Glasübergangstemperatur der Speichermodul verschwindet. Somit sollte dann für $T>>T_{\mathrm{g}}$ die Frequenzverschiebung nur durch die zusätzliche träge Masse auf dem Reed bedingt sein. Diese Annahme ist für unvernetzte Polymere, die bei hohen Temperaturen vollständig flüssig werden, sicherlich zulässig. In wie weit sie auch für die hier verwendeten laserdeponierten Filme legitim ist, wird in Kapitel 6.2 diskutiert.

Obwohl die Bestimmung des Speichermoduls also letztlich von der sehr präzise zu bestimmenden Verschiebung der Resonanzfrequenz abhängt, ist die Messung doch stark fehlerbehaftet, da die Biegesteifigkeit des Substrates sehr viel größer ist als die des Films. Die tatsächlich gemessene relative Änderung bleibt somit sehr klein und fehlerbehaftet.

Bedeutend genauer kann der Verlustmodul des Films bestimmt werden. Hier ist der die Auflösung limitierende Faktor die Güte der Schwingung des leeren Biegebalkens. Mit einem dem aus Kapitel 4.1.1 ähnlichem Formalismus ergibt sich:

$$
E_{\text {Film }}^{\prime \prime}=Q_{\text {Film }}^{-1} E_{\text {Film }}^{\prime}=\left(Q_{\text {Substrat }+ \text { Film }}^{-1}-Q_{\text {Substrat }}^{-1}\right) E_{\text {Substrat }}^{\prime} \frac{t_{\text {Substrat }}}{3 t_{\text {Film }}} . \quad \text { Formel } 4.33
$$




\section{Laserdeposition: Prozess und Eigenschaften}

Unter der Annahme, dass die Polymere wie Metalle bis auf atomare Ebene zerlegt würden (Vgl. Kapitel 3.1), wäre auszuschließen, dass sich bei der Laserdeposition ein Film bestehend aus dem komplexen und langkettigen Ausgangsmaterial bilden könnte. Auch die hohen Temperaturen von über $10.000 \mathrm{~K}$ im Ablationsplasma eines Metalls zerstörten sicherlich jegliches organisches Material. Es muss hier also ein grundlegend verschiedener Prozess vorliegen, der nur mit einem umfassenden Verständnis des Ablationsmechanismus aufgeklärt werden kann. Aufgrund der immensen technologischen Bedeutung z. B. auf dem Gebiet der MikroMaterialbearbeitung wurden die Grundzüge dieses Ablationsprozesses für PMMA bereits eingehend untersucht. Sie sind aber immer noch im Fokus der wissenschaftlichen Diskussion; eine zusammenfassende Darstellung dazu findet sich z. B. bei Dyer [101] oder Chrisey [4]. Im Folgenden soll geklärt werden, in wie weit sich die bekannten Konzepte auf die Mechanismen der Deposition der höheren Poly(alkyl methacrylat)e, zunächst PEMA, übertragen lassen.

\subsection{Morphologie}

Abbildung 5.1 zeigt elektronenmikroskopische Aufnahmen der Oberflächenmorphologie einer PEMA-Schicht, die mit 300 Laserpulsen auf Silizium deponiert wurde. Auffallend ist die im Vergleich zu metallischen Filmen sehr hohe Dichte an Tröpfchen (Droplets). Die Morphologie entspricht damit der einer vergleichbaren PMMA-Schicht (Vgl. z. B. [102]), s. d. ein erster Hinweis auf einen vergleichbaren Depositionsprozess gegeben ist. Die Inseln haben Durchmesser zwischen 0,2 und $10 \mu \mathrm{m}$ und weisen teilweise eine sehr flache, zylinderförmige Struktur, teilweise halbkugelartige Formen auf. Analog zu den von der Laserdeposition von Metallen bekannten Droplets [103-105] haben sie ihren Ursprung in Material, das in flüssiger Form vom Target zum Substrat transferiert wird und hier erstarrt. Ebenso wie die metallischen Droplets bilden sie, abhängig von Geschwindigkeit, Temperatur und Abkühlrate, am Substrat flache (ca. $200 \mathrm{~nm}$ ) oder eher hohe Strukturen mit Höhen bis zu $600 \mathrm{~nm}$ aus. Dennoch beruht 
ihre Bildung nicht, wie bei metallischen Schichten, auf einer Targetalterung, also der Aufrauhung der Targetoberfläche während der Laserdeposition, sondern sie treten bereits während der ersten Pulse auf ein frisches Target auf.
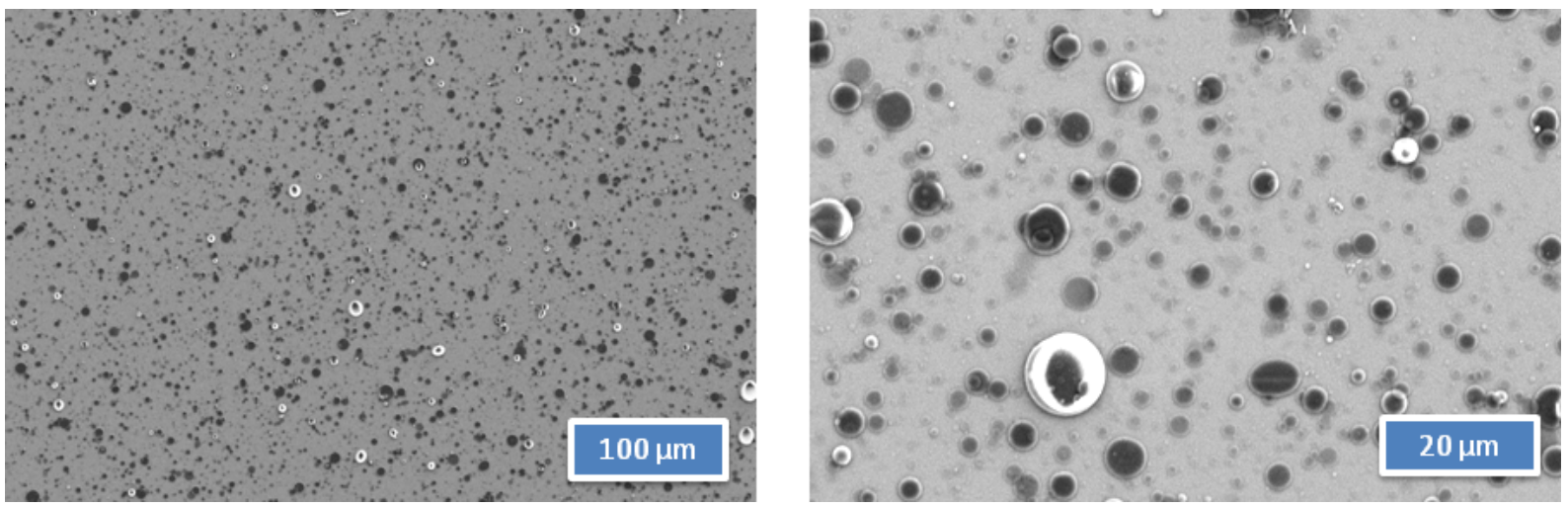

Abbildung 5.1: Elektronenmikroskopische Aufnahmen einer mit 300 Pulsen laserdeponierten PEMA-Schicht auf Silizium zeigen eine hohe Dropletdichte.

Der Inhalt dieser Tröpfchen lässt sich mit organischen Lösungsmitteln wie Tetrahydrofuran (THF) lösen. Auf der Substratoberfläche bleibt dann, wie Süske schon für PMMA zeigen konnte, unlösliches, größtenteils vernetztes Material zurück [15]. Dieser Rückstand ist in Abbildung 5.2 für eine mit 20.000 Pulsen hergestellte und dann mit THF behandelte PEMASchicht dargestellt. Besonders in der hoch vergrößerten Aufnahme rechts sind klar leere Hüllen zu erkennen, die vor dem Lösen eine Art Haut um die Droplets bildeten.
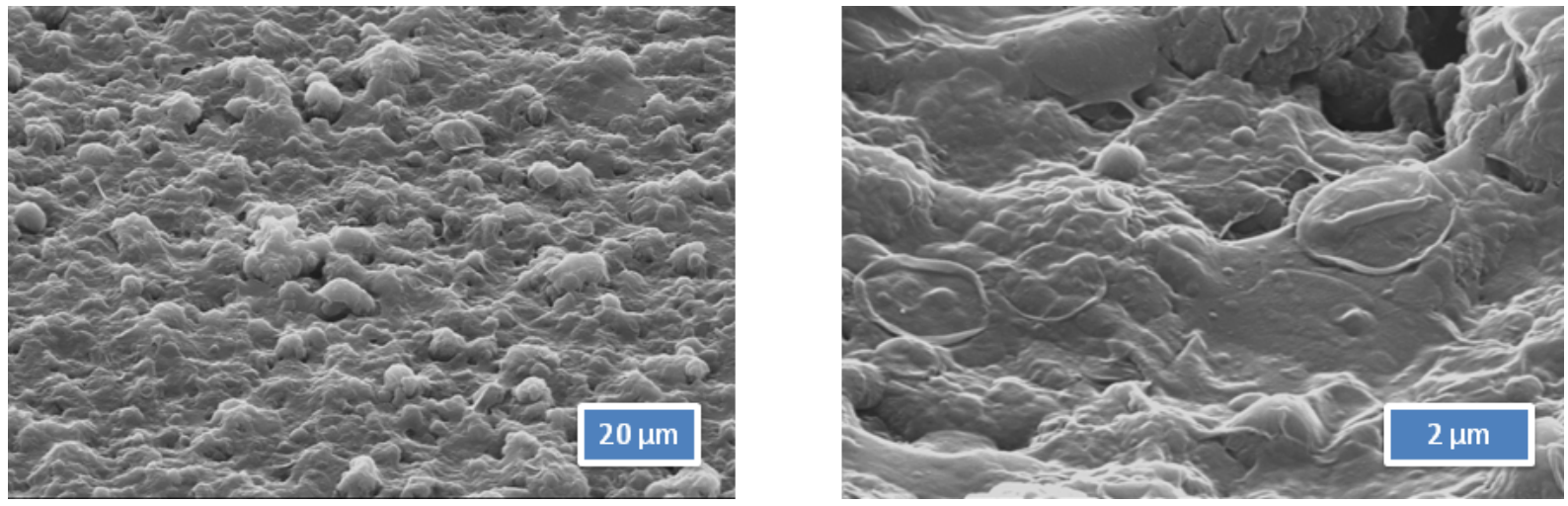

Abbildung 5.2: Elektronenmikroskopischen Aufnahmen des Rückstands einer mit 20.000 Pulsen laserdeponierten PEMA-Schicht auf Silizium nach dem Lösen in THF bei unterschiedlicher Vergrößerung.

Neben den oben gezeigten Droplets existiert also auch beim PEMA noch eine zweite Komponente, die z. B. mit der Röntgenreflektometrie zu untersuchen ist. Abbildung 5.3 zeigt Röntgenreflektogramme zweier PEMA-Schichten unterschiedlicher Schichtdicke. Die untere Kurve wurde dabei an der Probe aufgenommen, deren Oberflächenmorphologie auch in 
Abbildung $5.1 \mathrm{zu}$ sehen ist. Der schwache Abfall und die klar ausgeprägten Schichtdickenoszillationen (Kiessig-Fringes) lassen auf eine homogene Schichtdicke und sehr geringe Oberflächenrauigkeit der Schichten schließen [106]. Zusätzlich eingetragen sind mittels der Software IMD angefertigte Simulationen [80], die die Angabe von Massendichte, Schichtdicke, Rauigkeit und Dichte erlauben. Die entsprechenden Anpassungsparameter finden sich in Tabelle 5.1 und bestätigen den ersten Eindruck von sehr glatten Schichten. Die Rauigkeiten der glatten Komponente liegen für beide Schichten schichtdickenunabhängig bei etwa $0,3 \mathrm{~nm}$, was im Vergleich mit den elektronenmikroskopischen Aufnahmen zunächst verwundert. Diese scheinbare Unstimmigkeit der Morphologie im Rasterelektronenmikroskop und der Ergebnisse der Röntgenuntersuchung liegt darin begründet, dass der Einfluss der Inseln aufgrund ihrer stark unterschiedlichen Dicken für die XRR nicht zugänglich ist und somit nur der Anteil der zweiten, glatten Komponente gemessen werden kann. Die Höhen und die Höhenunterschiede der Droplets sind zu groß, als dass sie von der Kohärenzlänge der Röntgenstrahlung erfasst werden könnten.

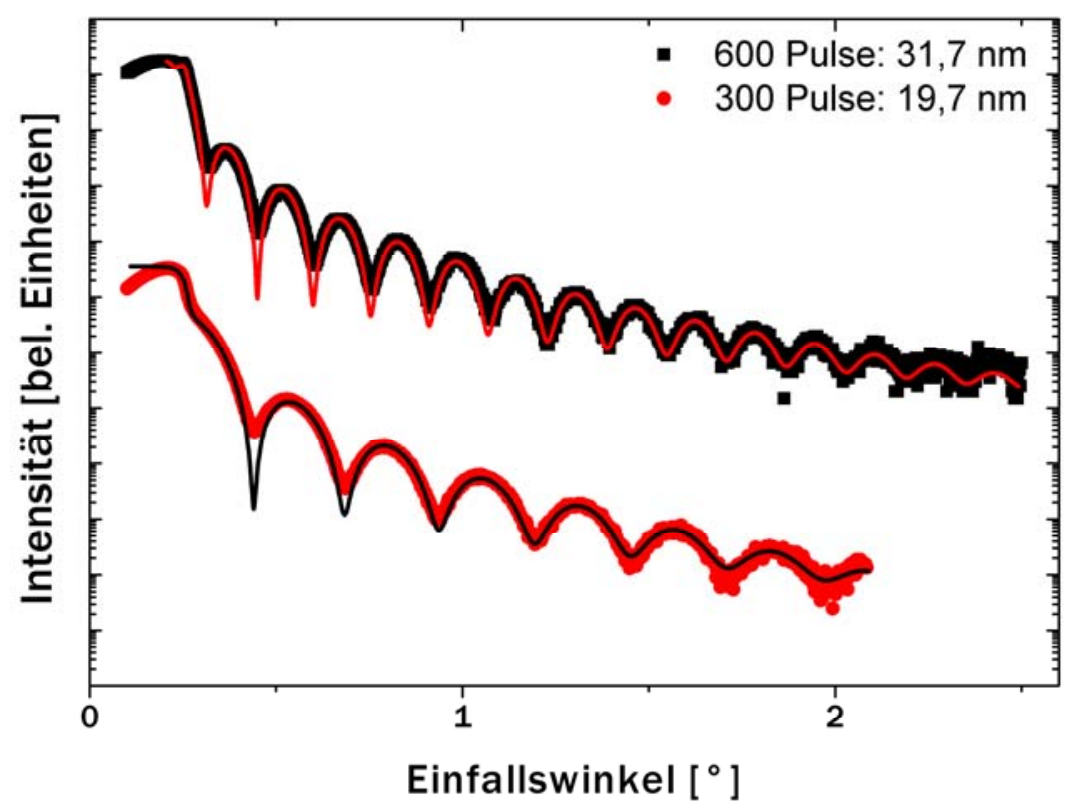

Abbildung 5.3: Die Röntgenreflektogramme für zwei Schichten unterschiedlicher Dicke lassen auf einen sehr glatten Schichtanteil schließen. Die angegebenen Schichtdicken dieser glatten Komponente wurden über die ebenfalls eingezeichneten IMD-Simulationen bestimmt.

Die typische Depositionsrate dieser glatten Komponente kann hier zu 0,06 nm/Puls bestimmt werden. Im Vergleich mit der Depositionsrate der Gesamtmasse, die in Kapitel $6.2 \mathrm{zu}$ $0,28 \mathrm{~nm} /$ Puls bestimmt wird, macht die glatte Komponente also etwa ein Fünftel der Gesamtmasse der Schichten aus. 


\begin{tabular}{|ccccc|}
$\begin{array}{c}\text { Anzahl Pulse } \\
\mathbf{n}\end{array}$ & $\begin{array}{c}\text { Dichte } \\
\boldsymbol{\rho}\left[\mathrm{g} / \mathrm{cm}^{3}\right]\end{array}$ & $\begin{array}{c}\text { Dicke } \\
\boldsymbol{d}[\mathrm{nm}]\end{array}$ & $\begin{array}{c}\text { Substrat-Rauigkeit } \\
\boldsymbol{\sigma}_{\text {Substrat }}[\mathrm{nm}]\end{array}$ & $\begin{array}{c}\text { Oberflächen-Rauigkeit } \\
\boldsymbol{\sigma}_{\text {Oberfläche }}[\mathrm{nm}]\end{array}$ \\
\hline 600 & 1,22 & 31,7 & 0,66 & 0,32 \\
\hline 300 & 1,22 & 19,7 & 0,71 & 0,30 \\
\hline
\end{tabular}

Tabelle 5.1: Mittels IMD bestimmte Anpassungsparameter für die in Abbildung 5.3 abgebildeten Simulationen.

Laserdeponierte Poly(alkyl methacrylat)e, die mit den für diese Arbeit typischen hohen Laserfluenzen von etwa $5 \mathrm{~J} / \mathrm{cm}^{2}$ hergestellt wurden, sind also stets zweikomponentige Proben, die sich aber z. B. durch chemische Lösungsmittel trennen lassen. Im Folgenden wird der lösbare Inhalt der Inseln als Tröpfchenanteil oder Dropletkomponente und der unlösliche Rest als glatter Filmanteil bezeichnet. Die Angabe „Film“ bezieht sich auf das komplette zweikomponentige System.

\subsection{Struktur und Zusammensetzung}

In Abbildung 5.4 ist das Röntgendiffraktogramm für einen PEMA-Film im Vergleich zum Ausgangsmaterial dargestellt. Beide Kurven zeigen ein charakteristisches breites Maximum unter einem Winkel von etwa $11^{\circ}$, was auf eine amorphe Struktur mit charakteristischen $\mathrm{Ab}$ ständen von ca. $0,7 \mathrm{~nm}$, sowohl im Targetmaterial als auch in der Schicht schließen lässt. Bei kleinen Winkeln ist bei beiden Kurven eine Flanke angedeutet. Diese wird in der Literatur einer Ordnung der Seitengruppen zugeordnet [107], die bei den Poly(alkyl methacrylaten) mit längeren Seitengruppen noch stärker ausgeprägt ist. Die Kurven für Schicht und Target zeigen einen ähnlichen Verlauf, die Proben weisen also eine ähnliche Nahordnung auf. Dennoch zeigen sich zu größeren Winkeln nur beim Diffraktogramm des Targets weitere Strukturen auf der rechten Flanke des Maximums. Diese Beiträge werden in der Literatur auf intra- bzw. intersegmentelle Abstände zwischen Hauptketten- und Seitengruppensegmenten zurückgeführt. Die Tatsache, dass diese im Diffraktogramm der Schicht fehlen, lässt den Schluss zu, dass hier eine breitere Verteilung von Abständen vorliegt, was wiederum durch die verringerte Kettenlänge zu erklären ist. 


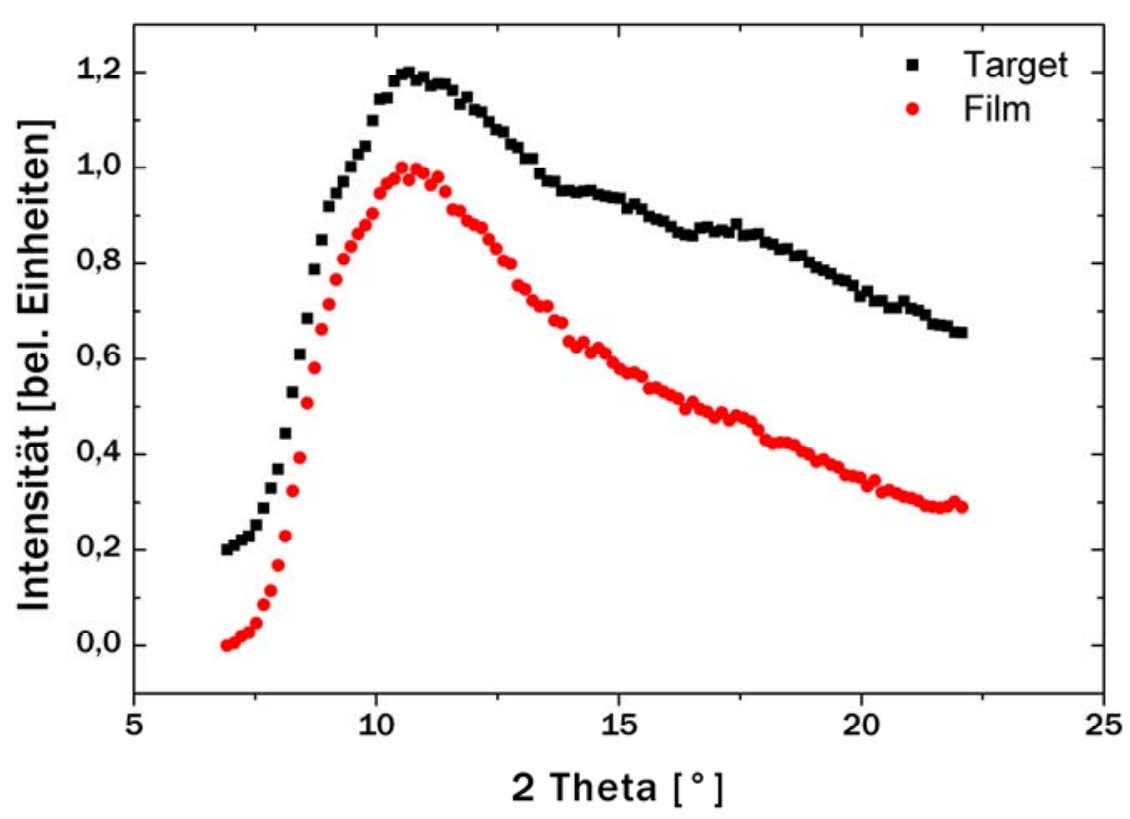

Abbildung 5.4: Die Röntgendiffraktogramme von PEMA-Target und -Schicht weisen auf eine amorphe Struktur hin. Die Ähnlichkeit der Form der Kurven lässt auf eine ähnliche Nahordnung von Target und Schicht schließen. Zur Übersichtlichkeit sind die Kurven in der Intensität verschoben.

In Abbildung 5.5 sind die FT-IR-Spektren eines PEMA-Targets sowie einer auf einem Aluminiumsubstrat laserdeponierten PEMA-Schicht dargestellt. Die Spektren weisen identische Absorptionsbanden auf, was auf sehr ähnliche chemische Bindungsverhältnisse schließen lässt. Alle charakteristischen Schwingungsbanden, die in Tabelle 5.2 notiert sind, sind vorhanden. Zum Vergleich ist zusätzlich das Spektrum einer laserdeponierten PMMA-Schicht eingezeichnet. Auch dieses ähnelt in weiten Bereichen dem der PEMA-Schicht. Lediglich zwischen 2900 und $2950 \mathrm{~cm}^{-1}$ sind zusätzliche Banden zu erkennen, die auf ValenzSchwingungen der zur Ethylester gehörenden $\mathrm{CH}_{2}$-Einheit zurückzuführen sind. Die obige Annahme der guten Vergleichbarkeit des Ablationsprozesses innerhalb der PAMA-Familie wird also erneut bestätigt.

Zur Untersuchung der Molmassenverteilung des deponierten Materials wurde in Zusammenarbeit mit dem Institut für Physikalische Chemie der Universität Göttingen die Größenausschlusschromatographie benutzt. Da dieses Verfahren gelöste Proben benötigt, sind hier nur Aussagen über die Dropletkomponente möglich, der unlösliche Rest wird nicht berücksichtigt. 


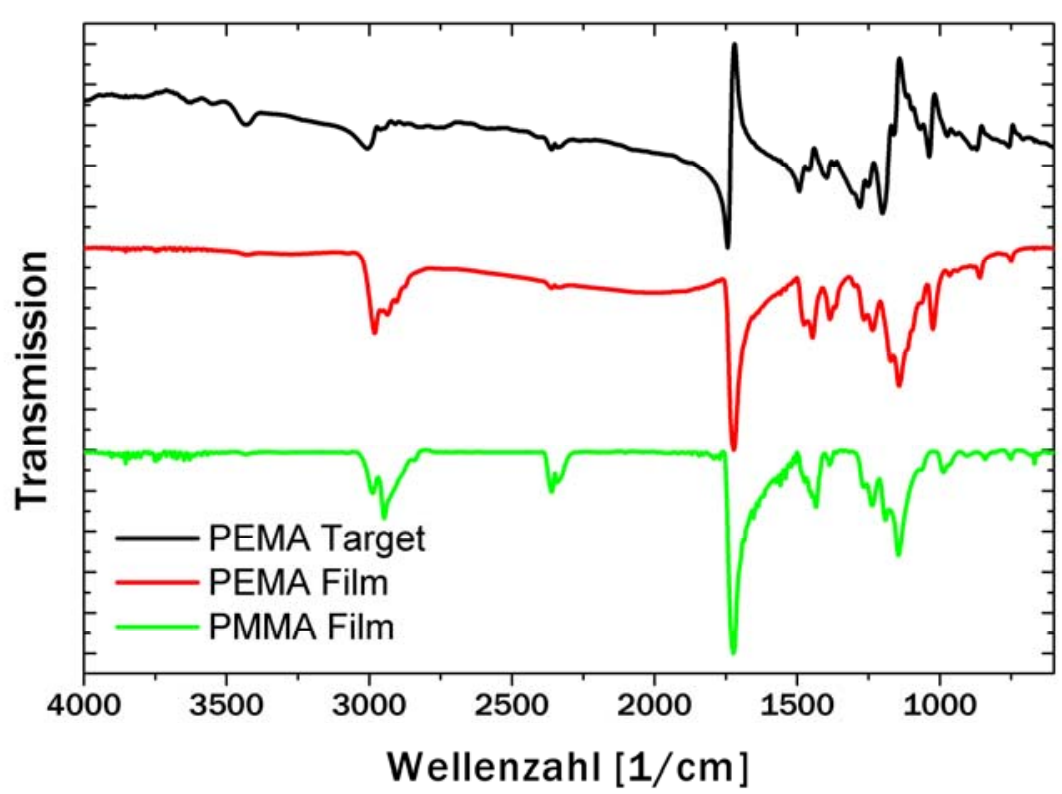

Abbildung 5.5: Infrarotspektren eines laserdeponierten PEMA-Films und des zugehörigen Targetmaterials. Zum Vergleich ist zusätzlich das Spektrum eines laserdeponierten PMMA-Films eingetragen. Die Abweichungen zwischen 2900 und $2950 \mathrm{~cm}^{-1}$ beruhen auf zusätzlichen $\mathrm{CH}_{2}$-Valenzschwingungsbanden der Ethylgruppe.

\begin{tabular}{|c|c|}
\hline $\begin{array}{l}\text { Wellenzahl } \\
v[1 / \mathrm{cm}]\end{array}$ & Zuordnung und Art der Schwingung \\
\hline 2985 & $v_{\mathrm{a}}\left(\mathrm{CH}_{2}\right)$ : antisymmetrische Valenzschwingung \\
\hline 2938 & $v_{\mathrm{s}}\left(\mathrm{CH}_{2}\right)$ : symmetrische Valenzschwingung \\
\hline 1729 & $v(\mathrm{C}=\mathrm{O}):$ Streckschwingung \\
\hline 1430 & $\delta_{\mathrm{s}}\left(\mathrm{CH}_{3}-\mathrm{O}\right)$ : symmetrische Deformationsschwingung um den Valenzwinkel \\
\hline 1190,1147 & Skelett-Valenzschwingungen gekoppelt mit CH-Deformationsschwingungen \\
\hline 1057 & Intramolekulare Wechselwirkung \\
\hline 747 & $\gamma_{\mathrm{r}}\left(\mathrm{CH}_{2}\right)$ : Torsionsschwingung gekoppelt mit Skelett-Valenzschwingung \\
\hline
\end{tabular}

Tabelle 5.2: Zuordnung der FT-IR Banden zu molekularen Schwingungen nach Dechant [108].

Abbildung 5.6 zeigt die so bestimmten Chromatogramme für das in THF lösbare Targetausgangsmaterial und den löslichen Anteil einer laserdeponierten PEMA-Schicht. Die massenmittlere Molmasse der Dropletkomponente ist mit $\bar{M}_{W}=8,7 \cdot 10^{3} \mathrm{~g} / \mathrm{mol}$ im Vergleich zum Ausgangsmaterial mit $\bar{M}_{W}=3,2 \cdot 10^{5} \mathrm{~g} / \mathrm{mol}$ stark reduziert. Gleichzeitig sinkt durch die Laserdeposition die Polydispersität $D=\bar{M}_{W} / \bar{M}_{n}$ des Polymers von $D_{\text {Target }}=2,9$ auf $D_{\text {Droplet }}=1,7$. Die Breite der Molmassenverteilung nimmt also sehr stark ab. Ähnliches Verhalten wurde auch für PMMA gefunden. Hier nimmt die massenmittlere Molmasse durch die Prozesse während der Laserdeposition auf $\bar{M}_{W}=8,0 \cdot 10^{3} \mathrm{~g} / \mathrm{mol} \mathrm{ab}[102]$. 


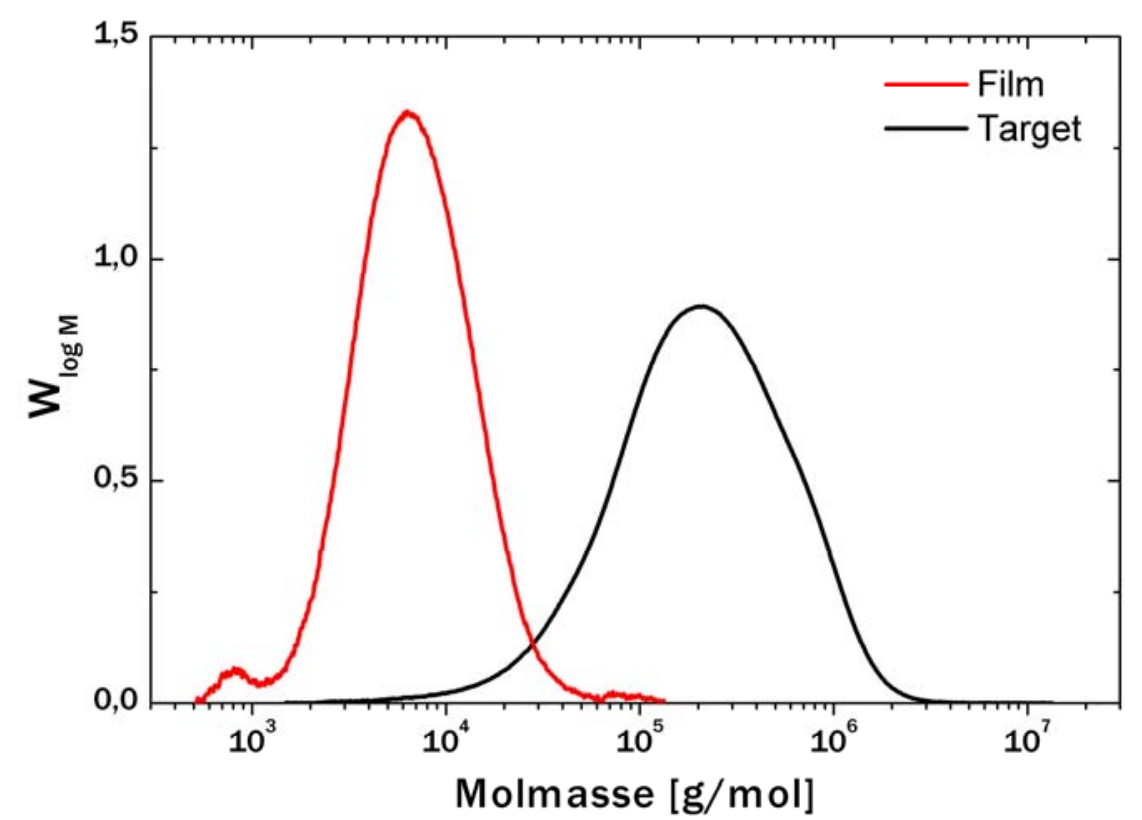

Abbildung 5.6: Chromatogramm eines laserdeponierten PEMA-Films im Vergleich mit dem des Ausgangsmaterials. Die molare Masse des Films ist durch die Laserdeposition stark gesunken. Gleichzeitig sinkt die Polydispersität von $D_{\text {Target }}=2,9$ auf $D_{\text {Droplet }}=1,7$.

\subsection{Ablations- und Depositionsmechanismus}

Die obigen Ergebnisse zeigen, dass während der Laserdeposition das Targetmaterial verändert und die Kettenlänge auf etwa $9.000 \mathrm{~g} / \mathrm{mol}$ reduziert wird. Weiterhin wird der zweikomponentige Charakter der Schichten, sie bestehen aus einer glatten und einer Tröpfchenkomponente, deutlich. Somit führen alle bisherigen Untersuchungen an PEMA-Schichten zu Ergebnissen, die denen von laserdeponierten PMMA-Schichten sehr ähnlich sind. Da auch die später beschriebenen Poly(butyl methacrylat)-Schichten vergleichbare Eigenschaften zeigen, ist es hier naheliegend, auch für die höheren Mitglieder der Poly(alkyl methacrylat)e einen verwandten zu Grunde liegenden Ablations- und Depositionsmechanismus anzunehmen.

Zusammen mit den Untersuchungen an PMMA von Süske [15] und Scharf [16] kann ein photochemischer Inkubationsprozess für eine chemische Veränderung des Targets verantwortlich gemacht werden. Dieser ist notwendig, bevor über photothermische Prozesse ein nennenswerter Abtrag stattfinden kann. Die Absorption im Targetmaterial wird dabei soweit erhöht, dass die Energie des Laserstrahls in einem ausreichend geringen Volumenanteil des Targets absorbiert werden kann. Reines PMMA beispielsweise hat eine Eindringtiefe von $150 \mu \mathrm{m}$ [109], was für direkten thermischen Abtrag zu groß ist. Mit $5 \mathrm{eV}$ ist die Photonenenergie des Excimerlasers aber ausreichend, um die meisten kovalenten Einfachbindungen im Material zu 
brechen (3-3,6 eV für die C-C Bindungen [110]). Die Laserstrahlung koppelt allerdings nicht direkt in die Einfachbindungen ein, sondern nutzt zunächst als Chromophore die Doppelbindung der Carbonylgruppe [111]. Diese Bindungen sind mit mittleren Bindungsenergien von 7,3 eV allerdings zu stark [112], um in einem Einzelphotonenprozess tatsächlich gebrochen zu werden. Stattdessen wird die Energie an eine benachbarte Einfachbindung weitergegeben, s. d. diese gebrochen und so z. B. die Ester-Seitengruppe abgespalten oder zumindest verändert werden kann. Dadurch kann es auch zu einer Spaltung der Hauptkette kommen, was zur Verringerung der mittleren Kettenlänge und zu einem Einbau zusätzlicher $\mathrm{C}=\mathrm{C}$ Doppelbindungen mit mittleren Bindungsenergien von $6,4 \mathrm{eV}$ führt [110].

Diese Doppelbindungen wirken als neue Chromophore und erhöhen die Absorption der UVStrahlung im Polymer [12] und somit die effektiv eingebrachte Energiedichte. Während die Inkubation bei der Verwendung von geringen Laserfluenzen tatsächlich vom Abtrag getrennt werden kann und dann äußerlich nur zur Aufrauung der Target-Oberfläche bzw. zu farblichen Veränderungen des Targets führt (Vergilben oder yellowing) [113, 114], führen die im Rahmen dieser Arbeit verwendeten Energiedichten bereits während des ersten Laserpulses zu einem Abtrag. Durch gleichzeitigen Abtrag von bereits verändertem Material und weiterer chemischer Modifikation stellt sich hier selbstorganisiert ein Gleichgewicht ein, bei dem der kritische Absorptionskoeffizient bei einer Molmasse von ca. $9.000 \mathrm{~g} / \mathrm{mol}$ erreicht wird. Zusätzlich führt der gleichzeitige Abtrag von Material mit ausreichend starker Absorption, also unterkritischer Molmasse, und die chemische Veränderung tieferer Schichten zu einer selbstorganisierten Regelung der Molmasse, was mit einer starken Reduktion der Polydispersität einher geht.

Die elektronenmikroskopischen Aufnahmen von Lösekrug in Abbildung 5.7 zeigen Poren unterhalb der Oberfläche eines beschossenen PMMA-Targets als Überreste des Ablationsvorgangs mit 800 Laserpulsen und einer Energiedichte von $5 \mathrm{~J} / \mathrm{cm}^{2}$ [113]. Sie erlauben ein tieferes Verständnis des stattgefundenen Ablationsvorgangs:

Durch die Inkubation wurde die Eindringtiefe zunächst auf etwa $10 \mu \mathrm{m}$ gesenkt, s. d. in einem Bereich etwa $5 \mu \mathrm{m}$ unter der Targetoberfläche Material stark erhitzt und verflüssigt, bzw. verdampft werden kann. Die flüssigen und gasförmigen Produkte dieser chemischen Veränderung und der teilweisen Zersetzung bilden blasenförmige Bereiche, die solange stabil sind, bis der Druck innerhalb der Blasen so stark ansteigt, dass die umgebende Hülle an der Targetoberfläche aufreißt. Das Innere der Blasen strömt unter hohem Druck strahlförmig aus den so 
entstandenen Löchern heraus und hinterlässt die in der Querschnittsaufnahme in Abbildung 5.7 sichtbaren Poren.

Über diese Kombination aus photochemischer Inkubation und photothermischem Abtrag lässt sich die Bildung der Dropletkomponente, also des mit etwa vier Fünfteln an der Gesamtmasse größten Anteils laserdeponierter PAMA-Filme, erklären.

Gleichzeitig entstehen durch photothermische (größenteils Abspaltung ganzer Monomereinheiten [115]), und photochemische Prozesse (Abspaltung und Zerlegung der Seitengruppe) kleinere Fragmente. Diese rekombinieren und repolymerisieren teilweise im Flug bzw. auf dem Substrat und führen hier zum glatten Filmanteil.
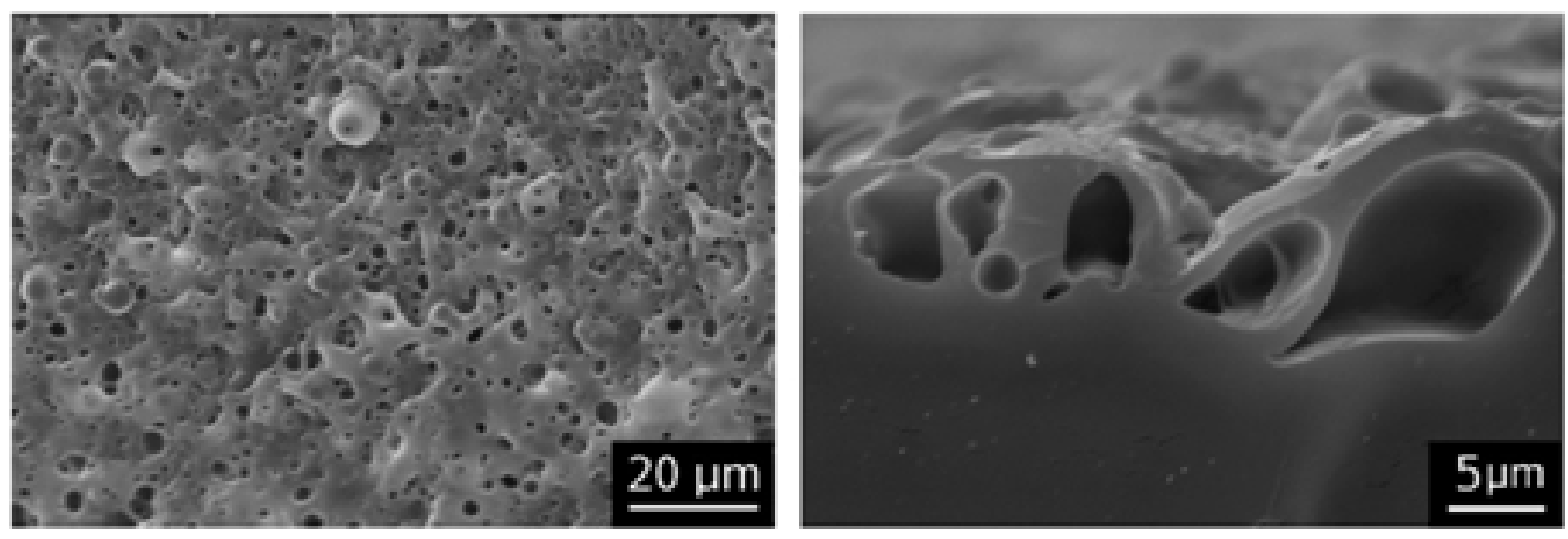

Abbildung 5.7: Die elektronenmikroskopischen Aufnahmen eines mit 800 Laserpulsen bei einer Energiedichte von $5 \mathrm{~J} / \mathrm{cm}^{2}$ bestrahlten PMMA Targets in Aufsicht und Querschnitt [113] zeigen Poren unter der Targetoberfläche, aus denen das flüssige Ursprungsmaterial der Droplets herausgeschleudert wurde. 


\section{Relaxation in Poly(ethyl methacrylat)}

Neben den morphologischen, chemischen und strukturellen Eigenschaften der polymeren Dünnfilme stehen besonders ihre internen Relaxationsmechanismen im Fokus dieser Arbeit. Komplementäre Untersuchungen mit verschiedenen spektroskopischen Methoden können hier neue Erkenntnisse liefern. Im Folgenden werden, zunächst an PEMA-Schichten, mechanische und dielektrische Eigenschaften bestimmt und es wird versucht, diese mit strukturellen Änderungen zu korrelieren. Die daraus resultierenden Ergebnisse werden anschließend in Kapitel 6.6 zusammenfassend diskutiert.

\subsection{Qualitativer Verlauf des Schermoduls}

Erste Versuche zur mechanischen Spektroskopie wurden an der in Kapitel 4.1.2 beschriebenen, im I. Physikalischen Institut vorhandenen, Anlage durchgeführt. Da diese Anlage nur zur Durchführung der spektroskopischen Messungen konzipiert ist und keine Beschichtungsmöglichkeit vorsieht, gestaltet sich die quantitative Bestimmung des Schermoduls hier als experimentell sehr aufwändig. Aufgrund der fehlenden in-situ Fähigkeit muss hier der DPO zunächst leer vermessen, dann aus der Spektroskopieanlage aus- und in die Beschichtungsanlage eingebaut werden, um schließlich wieder in der Spektroskopieanlage montiert zu werden. Daher wurde für die ersten der im Folgenden dargestellten Messungen auf eine Leermessung verzichtet. Für den zunächst diskutierten qualitativen Verlauf und insbesondere für relative Änderungen ist die Messung des leeren Oszillators nicht zwingend notwendig. Die hier dargestellten Graphen zeigen stattdessen zusätzlich die mit einem anderen DPO aufgenommenen Leermessungen.

Während der inverse Qualitätsfaktor des leeren im Vergleich zu dem des beschichteten Oszillators ohnehin sehr niedrig und somit in erster Näherung vernachlässigbar ist, kann die absolute Lage der Resonanzfrequenz der AS2-Mode unterschiedlicher Oszillatoren durchaus um 
einige $100 \mathrm{~Hz}$ verschoben sein. Trotz der Abweichungen in der absoluten Resonanzfrequenz bei verschiedenen Oszillatoren ist ihre Temperaturabhängigkeit jedoch auch für unterschiedliche Oszillatoren ähnlich.

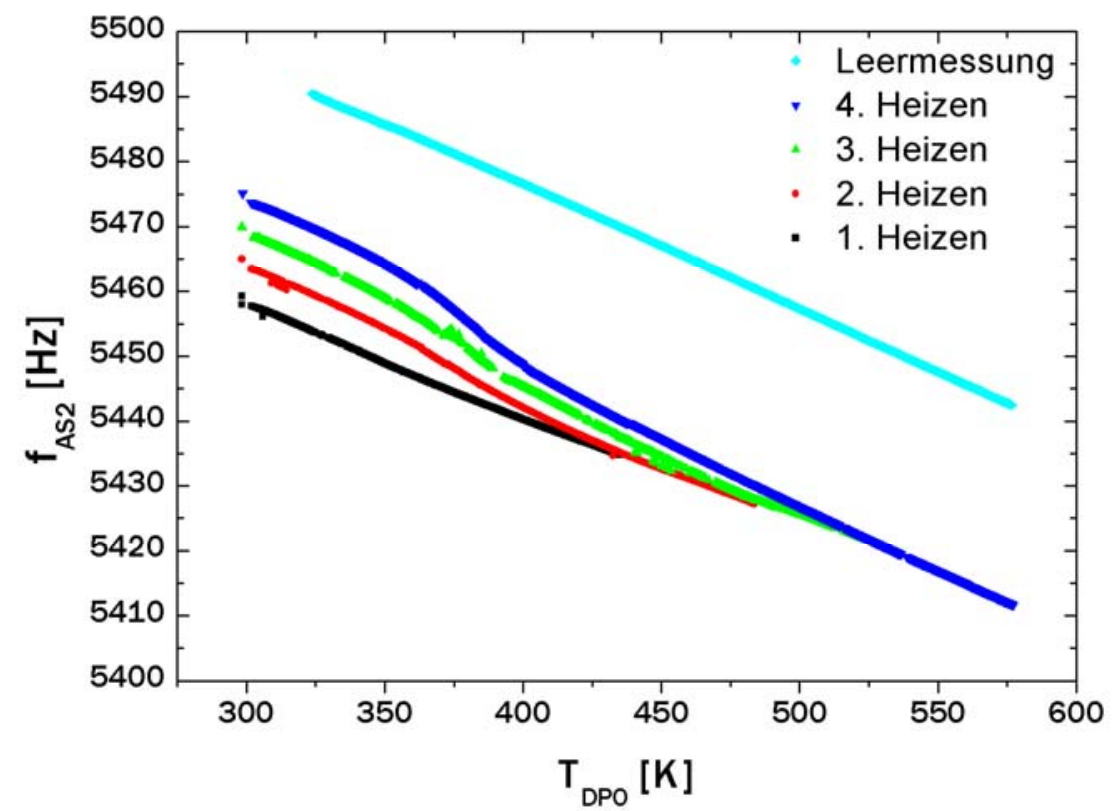

Abbildung 6.1: Temperaturabhängiger Verlauf der AS2-Resonanzfrequenz während vier sukzessiven Auslagerungszyklen einer mit 5.000 Pulsen deponierten PEMA-Schicht auf einem Doppelpaddeloszillator. Die eingezeichnete Leermessung dient nur zur Demonstration des linearen Frequenzverlaufs eines unbeschichteten DPOs und resultiert aus der Messung eines Vergleichsoszillators.

Abbildung 6.1 zeigt die Resonanzfrequenz der AS2-Mode eines mit 5.000 Laserpulsen PEMA beschichteten Doppelpaddeloszillators bei verschiedenen aufeinanderfolgenden Heizbehandlungen mit einer Heizrate von $0,3 \mathrm{~K} / \mathrm{min}$. Zum Vergleich ist oberhalb der Kurven der lineare Frequenzverlauf eines Referenzoszillators eingezeichnet. Beim ersten Aufheizen auf bis zu $435 \mathrm{~K}$ nach der Herstellung bei Raumtemperatur zeigt die Frequenzkurve zunächst einen linearen Verlauf, knickt aber bei etwa $365 \mathrm{~K} \mathrm{zu}$ einer leicht stärkeren negativen Steigung ab. Beim zweiten Aufheizen, nachdem die Probe wieder bis Raumtemperatur gekühlt wurde, zeigt sich ein nicht reversibles Verhalten. Bereits bei Raumtemperatur ist die Resonanzfrequenz etwa $6 \mathrm{~Hz}$ höher als bei der Messung an der frisch hergestellten Probe. Erneut bei etwa $365 \mathrm{~K}$ knickt der Verlauf mit stärkerer Steigung ab und nähert sich dann langsam der ersten Kurve an. Etwa beim Erreichen der maximalen Temperatur der ersten Messung bei $435 \mathrm{~K}$ berühren sich die beiden Kurven und die Neukurve setzt den extrapolierten Verlauf der ersten Kurve fort. 
Dieses Verhalten wiederholt sich auch für die weiteren Auslagerungen: Zunächst ist ein erster Knickpunkt bei etwa $365 \mathrm{~K}$ zu beobachten, dann nähern sich alle Proben dem extrapolierten Verlauf der ersten Kurve an. Der Punkt, an dem eine Folgemessung den Verlauf der vorherigen Messung berührt, entspricht dabei immer der maximalen Temperatur der vorigen Messung.

Gemäß Formel 4.19 tragen zwei Beiträge mit entgegengesetztem Vorzeichen zur Verschiebung der Resonanzfrequenz bei: Während die zusätzlichen elastischen Beiträge der Schicht eine Verschiebung zu höheren Frequenzen bewirken, wird die Resonanzfrequenz durch die zusätzliche Masse zu kleineren Werten verschoben. Die beobachtete auslagerungsbedingte Zunahme der Resonanzfrequenz bei Raumtemperatur kann also entweder durch eine Zunahme der elastischen Beiträge in der Schicht, also durch eine Erhöhung des Schermoduls des Polymers, oder durch Masseverlust erklärt werden. Thermogravimetrische Untersuchungen von laserdeponierten PMMA-Filmen zeigen, dass diese bereits bei $485 \mathrm{~K}$, also bei einer Temperatur etwa $35 \mathrm{~K}$ unterhalb der Degradationstemperatur von Volumenproben, beginnen zu zerfallen [15]. PEMA ist thermisch stabiler als PMMA [116], s. d. auch für die laserdeponierten Proben aus diesem Polymer thermische Stabilität bis mind. $485 \mathrm{~K}$ angenommen werden kann. Ein Masseverlust würde weiterhin zur Resonanzfrequenzverschiebung im gesamten Temperaturbereich führen, s. d. die unterschiedlichen Kurven nicht mehr stetig aneinander gesetzt werden könnten. Die Kurven tangieren sich aber, s. d. dieser Effekt hier auszuschließen ist und eine thermisch induzierte Zunahme des Schermoduls angenommen werden kann.

Die irreversible Veränderung des Polymers spiegelt sich auch in der Dämpfung wider. Abbildung 6.2 zeigt den Verlauf des inversen Qualitätsfaktors während der oben beschriebenen Messungen. Die Kurve der ersten Auslagerung liegt bei Raumtemperatur mit einer Dämpfung von $2,8 \cdot 10^{-5}$ etwa eine Größenordnung über der Leermessung und steigt bis $435 \mathrm{~K}$ auf etwa $1,0 \cdot 10^{-4}$ an. Während die Dämpfung der Leermessung im gesamten Temperaturbereich unterhalb von $2,2 \cdot 10^{-5}$ bleibt, führt die zusätzliche Schicht also zu einer starken Zunahme der Dämpfung bzw. des mechanischen Verlustes im Komplettsystem DPO-Schicht. Das zusätzlich auftretende Dämpfungsmaximum kennzeichnet den Glasübergang. Mit dem Anfang des Peaks bei $330 \mathrm{~K}$ ist die Glasübergangstemperatur festgelegt. Bei $370 \mathrm{~K}$ ist die Dämpfung mit $Q^{-1}=1,1 \cdot 10^{-4}$ maximal, bevor mit dem Ende der ersten Messung bei $435 \mathrm{~K}$ das Maximum überwunden ist. 


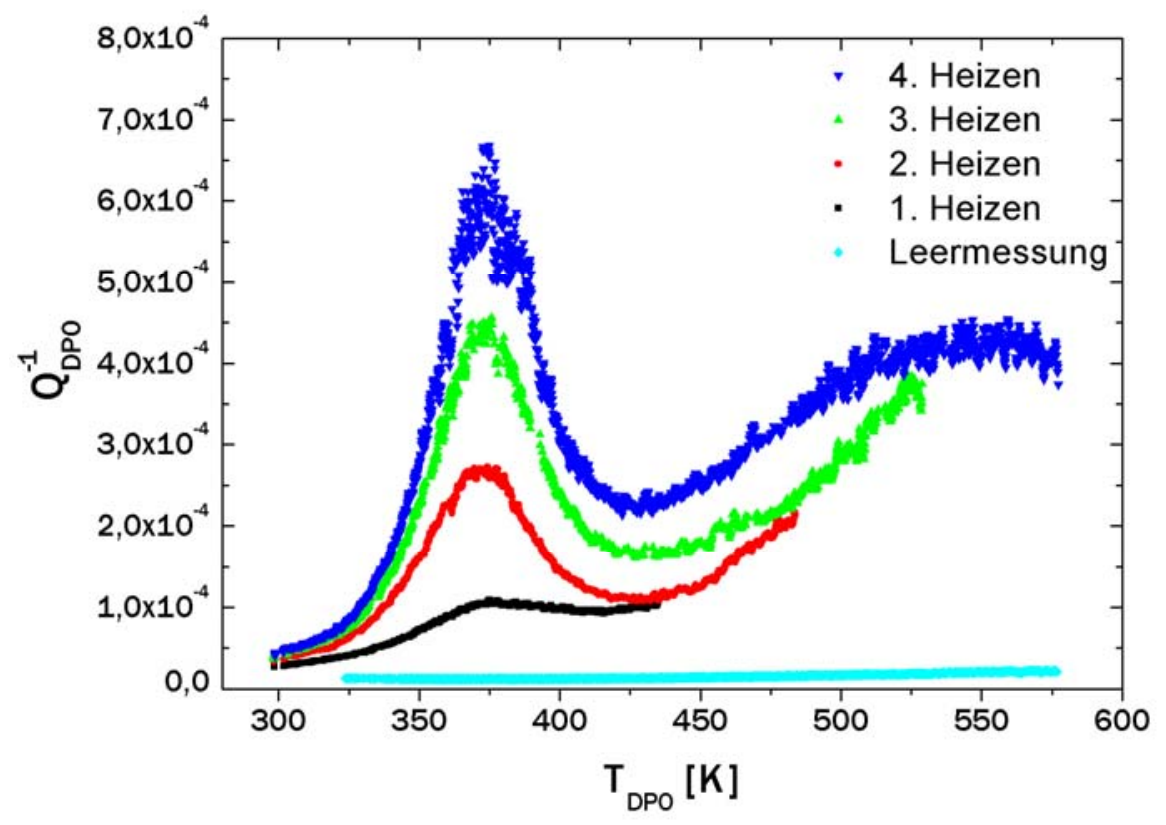

Abbildung 6.2: Temperaturabhängiger inverser Qualitätsfaktor für eine mit 5.000 Laserpulsen deponierte PEMASchicht bei vier sukzessiven Auslagerungen. Die eingezeichnete Leermessung ist mit einem Referenz-DPO aufgenommen und dient hier lediglich zur Illustration des qualitativen Verlaufs. Sie ist im Vergleich zu den Messwerten des beschichteten DPOs allerdings so gering, dass der Substratbeitrag hier vernachlässigt werden kann.

$\mathrm{Zu}$ Beginn des zweiten Heizdurchgangs ist die Dämpfung bei Raumtemperatur im Vergleich zur ersten Messung nur marginal erhöht. Das Maximum des Glasübergangs ist aber mit einer maximalen Dämpfung von $2,7 \cdot 10^{-4}$ deutlich verstärkt. Analog zu dem Verlauf der Resonanzfrequenzen treffen sich die Kurven der ersten und der Folgemessung bei der letzten maximalen Auslagerungstemperatur und die neuen Daten lassen sich stetig an die Daten der ursprünglichen Messung anschließen.

Beginnend mit dem dritten Heizdurchgang wird nicht nur die Dämpfung im Bereich des Glasübergangsmaximums stark verstärkt, sondern auch die bei höheren Temperaturen ab $425 \mathrm{~K}$. Auch die im dritten und vierten Heizdurchgang, also bei Messung an einer zuvor bei 465 K bzw. 505 K ausgelagerten Schicht, aufgenommenen Dämpfungen sind bei Raumtemperatur nur unwesentlich größer als die der ersten Messung. Bei höheren Temperaturen ist die Dämpfung jeder Folgemessung jedoch stärker ausgeprägt als die der vorigen Messung.

Dieses Verhalten ist in Abbildung 6.3 durch Bildung des Quotienten bzw. der Differenz jeweils zweier aufeinanderfolgender Messungen dargestellt. Im linken Teil der Abbildung wird deutlich, dass nach der ersten Auslagerung bis $435 \mathrm{~K}$ nur das zum Glasübergang gehörende Maximum verstärkt wird. Nach der zweiten Auslagerung bis $485 \mathrm{~K}$ tritt dann bei höheren 
Temperaturen zusätzliche Dämpfung auf. Dieser Effekt verstärkt sich nach der dritten Auslagerung bei $530 \mathrm{~K}$.
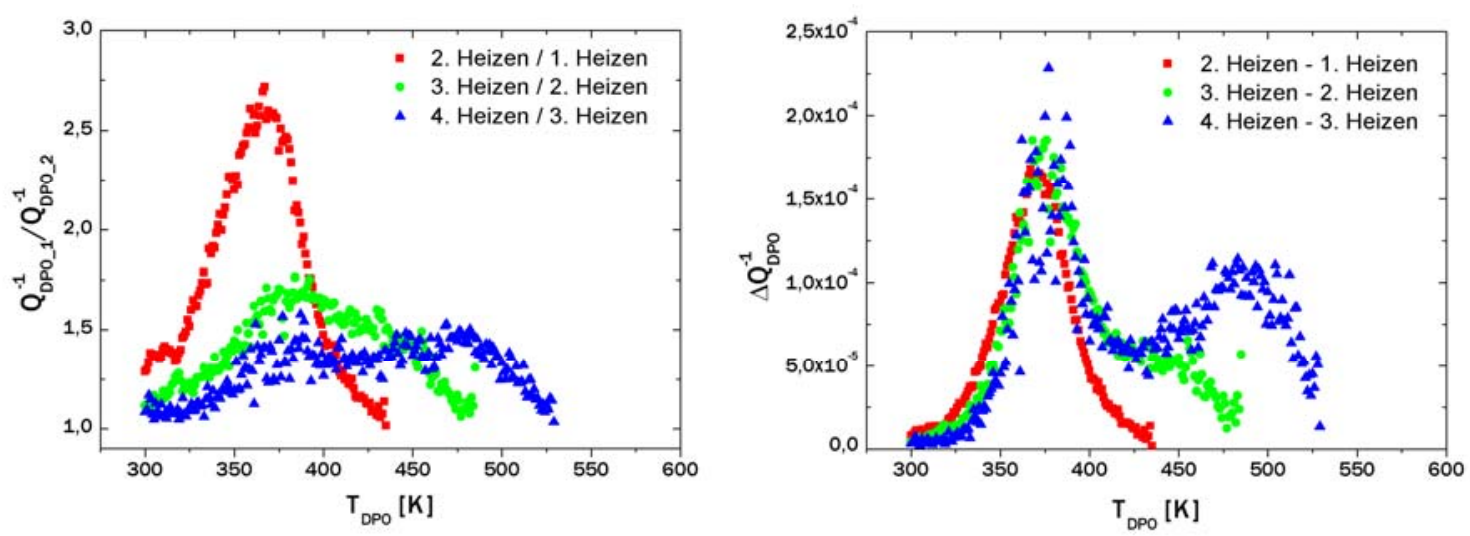

Abbildung 6.3: Relative (links) und absolute (rechts) Änderung des inversen Qualitätsfaktors zwischen jeweils zwei Messdurchläufen mit jeweils unterschiedlicher Endtemperatur. Nach der ersten Auslagerung bis $485 \mathrm{~K}$ ist besonders das Maximum des Glasübergangs verstärkt. Für höhere Auslagerungstemperaturen treten zusätzliche Dämpfungsprozesse im Bereich oberhalb des Glasübergangs auf.

Im rechten Teil von Abbildung 6.3 sind die absoluten Differenzen zwischen jeweils zwei aufeinanderfolgenden Messungen gezeigt. Es zeigt sich, dass durch die jeweilige Auslagerung bei einer äquidistant um 45 - $50 \mathrm{~K}$ erhöhten Temperatur eine ähnliche Verstärkung der Dämpfung im Bereich des Maximums auftritt. Mit weiterer Auslagerung bei höheren Temperaturen wird zusätzlich auch die Dämpfung bei Temperaturen oberhalb des Glasübergangs verstärkt. Die Dämpfung verschwindet also auch bei hohen Temperaturen nicht vollständig, sondern steigt auch oberhalb des Glasübergangsmaximums weiter an. Dies ist ein Zeichen für das Vorhandensein von vernetztem Polymeranteil in der Schicht. Das Polymer aus quervernetzten Makromolekülen wird auch bei hohen Temperaturen nicht komplett flüssig, sondern zeigt durch die Zunahme der Viskosität in starkem Maße duroplastischen Charakter [69].

Zur Beschreibung der Relaxationsprozesse, die zum Anwachsen der Intensität des Glasübergangsmaximums, also des $\alpha$-Peaks führen, sind in der Arrhenius-Auftragung in Abbildung 6.4 die logarithmierten Maximalwerte des inversen Qualitätsfaktors über der reziproken Temperatur aufgetragen. Man erkennt, dass die drei Messwerte, die zu den ausgelagerten Proben gehören, gut auf einer Gerade liegen. Dies lässt auf einen einfach thermisch aktivierten Prozess mit einer Aktivierungsenergie von 0,19 eV schließen. Diese Aktivierungsenergie ist vergleichbar mit der Aktivierungsenergie der radikalischen Polymerisation (z. B. 0,2 eV für PMMA [117]). Süske fand einen ähnlichen aktivierten Prozess als Ursache für das Anwachsen der mittleren 
Molmasse und der Härte laserdeponierter PMMA-Schichten bei Herstellung auf geheizten Substraten [15].

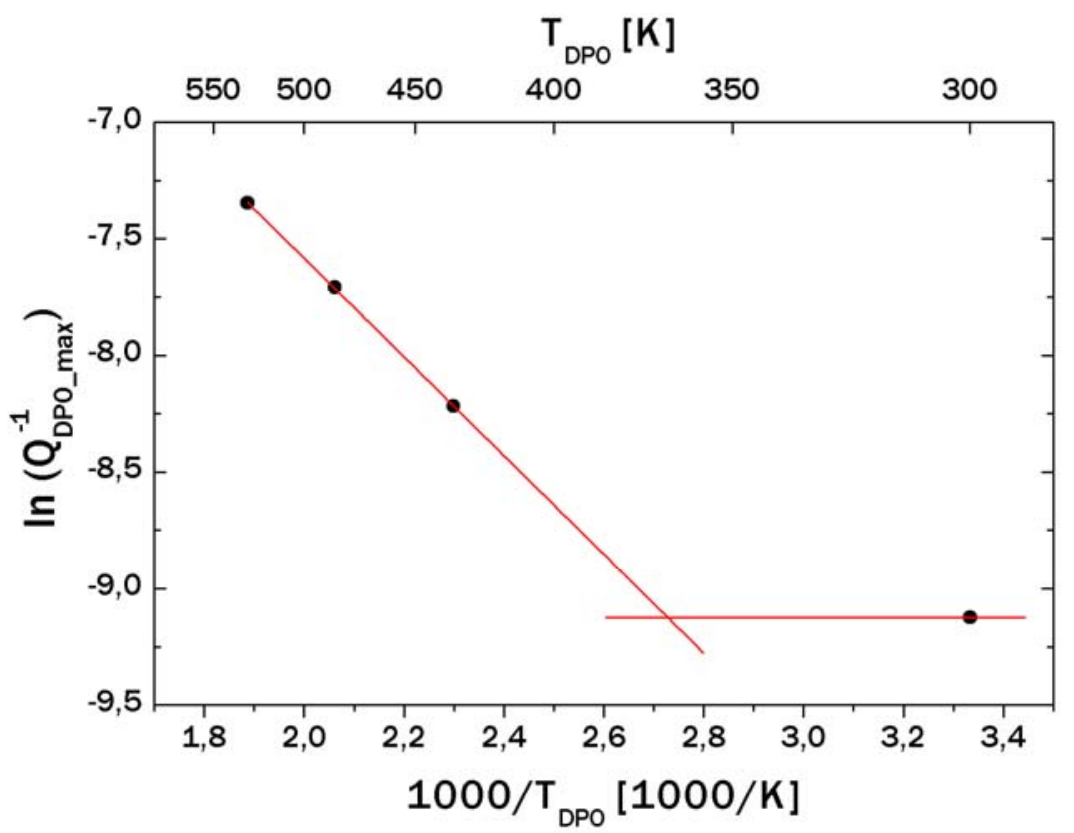

Abbildung 6.4: Die Arrhenius-Darstellung der maximalen Dämpfungswerte über der reziproken Temperatur lässt auf einen einfach thermisch aktivierten Prozess als Ursache für den Dämpfungsanstieg im Maximum schließen. Der Messwert für die bei Raumtemperatur ausgelagerte Probe weicht vom Arrhenius-Verhalten ab. Hier ist von einer Schwellentemperatur auszugehen, unter der keine Änderung im Material hervorgerufen wird.

Der Messwert der Erstmessung, also der noch nicht ausgelagerten Probe, passt nicht in diese Darstellung. Vielmehr erscheint hier die Annahme einer Schwellentemperatur, unter der keine Änderung im Material auftritt, sinnvoll. Gemäß der Rohdaten bzw. der Differenzen in Abbildung 6.3 liegt diese Temperatur im Bereich der Glasübergangstemperatur. Daher ist in Abbildung 6.4 zusätzlich eine horizontale Gerade auf Höhe des inversen Qualitätsfaktors der Raumtemperatur-Messung eingezeichnet. Diese schneidet die zu den ausgelagerten Proben gehörende Regressionsgerade bei 1000/T =2,7, also bei einer Auslagerungstemperatur von 366 K, was gerade der Temperatur des Dämpfungsmaximums entspricht. Dies ist ein Zeichen dafür, dass erst die durch den Glasübergang erhöhte Mobilität der Ketten, bzw. Kettensegmente eine irreversible Relaxation im Polymer zulässt, die dann zur Zunahme der Energiedissipation im Polymer führt. 


\subsection{Quantitative Bestimmung des Elastizitätsmoduls}

Mit dem Plasma Plume eXcited Reed (PPXR) steht als Erweiterung in Laserdepositionsanlagen eine sehr flexible Methode zur quantitativen Bestimmung des komplexen Elastizitätsmoduls zur Verfügung. Bedingt durch die Möglichkeit, in-situ Filme herzustellen und diese ohne Brechen des Vakuums vermessen zu können, ist es vergleichsweise einfach möglich, unterschiedliche Schichtdicken und Schichtkombinationen zu vermessen. Bei den ersten der hier gezeigten Messungen soll daher zunächst beispielhaft die Schichtdickenabhängigkeit der mechanischen Eigenschaften untersucht werden.

Dazu wurden die Proben vor der jeweiligen Messung auf eine Temperatur $10 \mathrm{~K}$ oberhalb der maximalen Messtemperatur erhitzt und hier für 10 Minuten ausgelagert. Somit kann zum einen der Effekt der Relaxation als Überlagerung der eigentlichen Messung ausgeschlossen werden, zum anderen können die nacheinander deponierten Schichten in einen vergleichbaren Zustand gebracht werden.

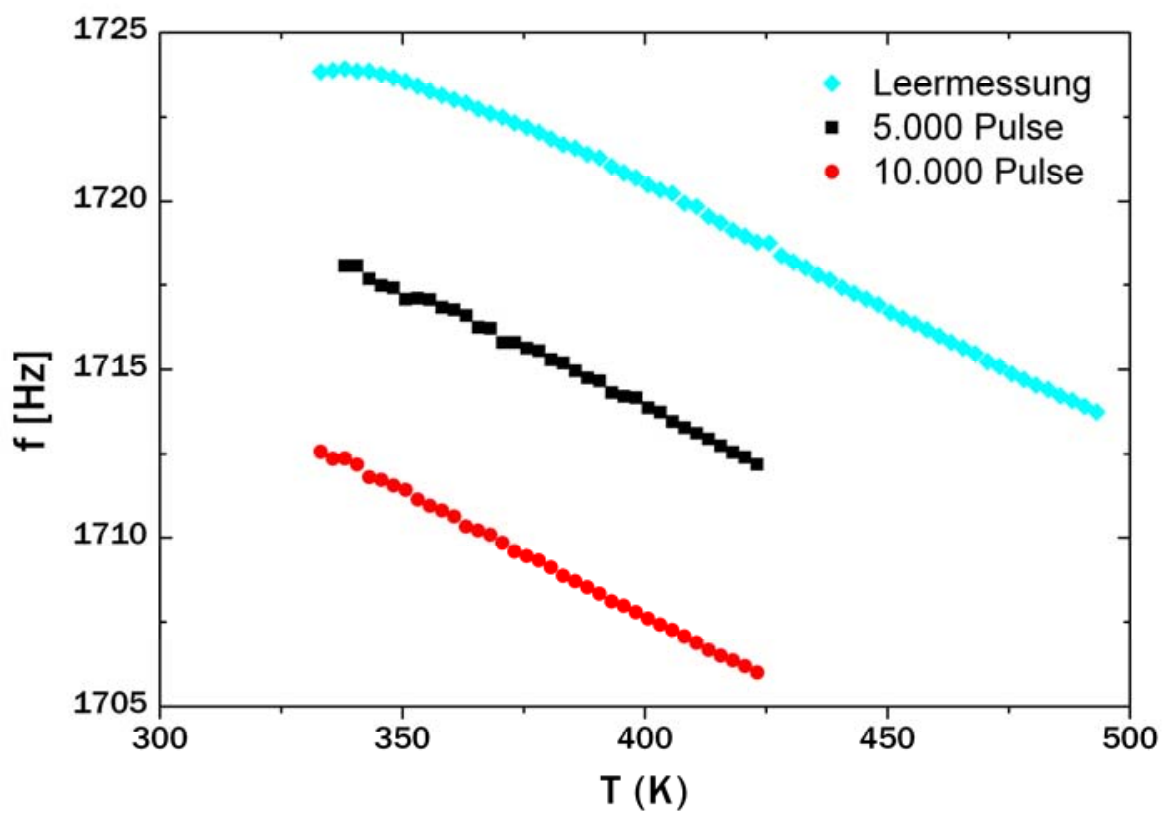

Abbildung 6.5: Mittels PPXR bestimmte temperaturabhängige Frequenzen für ein leeres Substrat und zwei PEMASchichten unterschiedlicher Dicke. Aus der Frequenzverschiebung lassen sich die Schichtdicken zu 1,43 $\mu \mathrm{m}$ und $2,79 \mu \mathrm{m}$ ableiten.

Abbildung 6.5 zeigt die mit PPXR gemessenen temperaturabhängigen Frequenzen für ein leeres etwa $9 \mathrm{~mm}$ langes Reed-Substrat, sowie zwei weitere Frequenzverläufe für das beschichtete Substrat. Dazu wurden nach der Leermessung zunächst 5.000 Pulse PEMA bei 
Raumtemperatur deponiert, das System auf $435 \mathrm{~K}$ geheizt und für 10 Minuten ausgelagert, wieder abgekühlt und schließlich vermessen.

Vor der zweiten Messung wurden bei Raumtemperatur weitere 5.000 Pulse PEMA deponiert und die Schicht abermals ausgelagert und abgekühlt. Die Resonanzfrequenz des leeren Substrates sinkt wie erwartet mit zunehmender Temperatur ab. Allerdings ist bei niedrigen Temperaturen eine Abweichung vom linearen Verhalten zu erkennen. Diese beruht sehr wahrscheinlich auf Problemen bei der Temperaturregelung, weil hier eine Regelung in der Nähe der Umgebungstemperatur schwierig ist.

Die Frequenzen der beschichteten Proben sind aufgrund der zusätzlichen Masse zu tieferen Werten verschoben. Mit der Annahme, dass für hohe Temperaturen der Speichermodul in der Schicht verschwindet, lässt sich aus der maximalen Frequenzverschiebung die Masse und somit die Schichtdicke des Filmes bestimmen. Die entsprechenden Schichtdicken ergeben sich zu 1,43 $\mu \mathrm{m}$ für die mit 5.000 Pulsen deponierte und $\mathrm{zu} 2,79 \mu \mathrm{m}$ für die mit insgesamt 10.000 Pulsen hergestellte Probe.

Die zugehörige Güte der Schwingung ist in Abbildung 6.6 aufgetragen. Hier zeigt die Leermessung über einen weiten Temperaturverlauf den erwarteten flachen Verlauf, allerdings tritt bei etwa $410 \mathrm{~K}$ ein leichtes Maximum auf, das vermutlich auf eine organische Kontamination des Substrats zurückzuführen ist. Für die 1,43 $\mu$ m dicke Probe zeichnet sich bei $350 \mathrm{~K}$ ein Maximum ab (schwarze Quadrate), die Streuung dieser Messung ist jedoch sehr hoch. Die Schwingungsqualität dieses Biegebalkens ist für eine aussagekräftige Messung mit dieser Schichtdicke also zu gering, s. d. im Folgenden nur die dickere Probe betrachtet wird.

Die Messung an der mit 10.000 Pulsen hergestellten Probe (rote Punkte) ist bedeutend weniger verrauscht und das zum Glasübergang gehörende Maximum bei $355 \mathrm{~K}$ ist gut zu erkennen. Dieses liegt im Vergleich zu der mit dem DPO gemessenen Dämpfung (Abbildung 6.2) bei um etwa $15 \mathrm{~K}$ geringeren Temperaturen. Ähnliche Abweichungen zeigen sich auch beim Vergleich von Messungen an PMMA von Rösner und Scharf und sind auf instrumentell bedingte Unterschiede von gemessener und tatsächlicher Temperatur zurückzuführen [16, 97]. Da die PPXR-Apparatur in diesem frühen Stadium noch nicht für die Messung unterhalb von $330 \mathrm{~K}$ optimiert war, ist die quantitative Analyse in diesem Temperaturbereich noch nicht möglich. Auch die Angabe der Onset-Temperatur des Maximums, also der Glasübergangstemperatur muss hier offen bleiben. Aus diesem Grund wird das Verhalten unterhalb von 
$330 \mathrm{~K}$ erst im folgenden Kapitel 6.3 am Beispiel weiterer DPO-Messungen ausgiebig behandelt. Alle folgenden Angaben über den Temperaturverlauf unter $330 \mathrm{~K}$ können daher stark fehlerbehaftet sein.

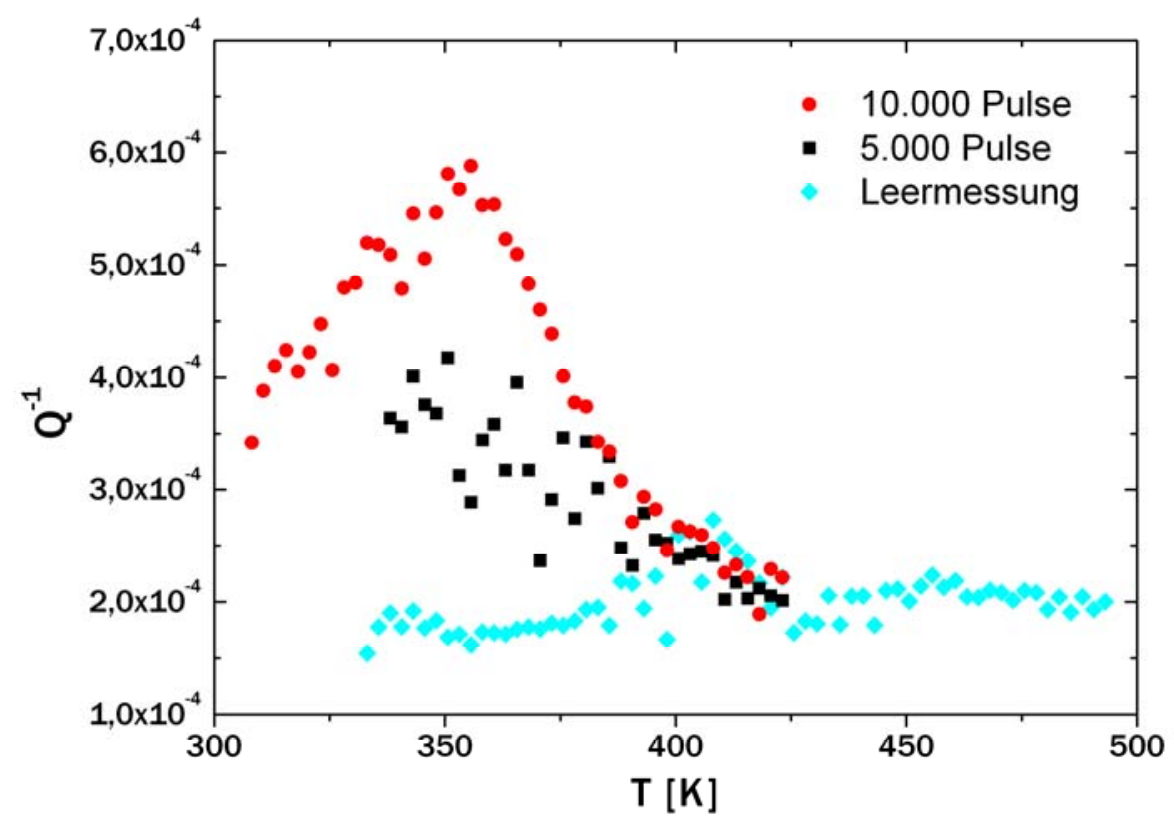

Abbildung 6.6: Temperaturabhängiger inverser Qualitätsfaktor für das leere Substrat und zwei unterschiedlich dicke PEMA-Schichten.

Zur zusätzlichen Untersuchung der bereits in Kapitel 6.1 diskutierten thermisch induzierten Relaxation im PEMA wurde die Probe nach diesen Messungen weiter vermessen. Sie wurde dabei vor der jeweiligen Messung nicht ausgelagert, sondern während der Messung erstmals auf höhere Temperaturen oberhalb von $425 \mathrm{~K}$ gebracht.

In Abbildung 6.7 ist die dabei bestimmte temperaturabhängige Frequenzverschiebung in Abhängigkeit vom vorigen Auslagerungszustand aufgetragen. Die Daten der roten Kurve entsprechen den Frequenzen der roten Kurve in Abbildung 6.5. Der Betrag der Frequenzverschiebung steigt zunächst mit der Temperatur an, was auf eine Abnahme der elastischen Rückstellkräfte in der Schicht aufgrund des Glasübergangs schließen lässt. Bei $400 \mathrm{~K}$ wird ein Minimum erreicht, nach dem die Frequenzverschiebung wieder abnimmt.

Der Vergleich mit der Folgemessung der Probe mit identischer Auslagerungstemperatur (grüne Kurve) zeigt nur geringe Unterschiede, allerdings zeigen die Werte systematisch eine geringe Verschiebung der Frequenz, was ein Anzeichen für eine nicht reversible Veränderung der Probe während des zweiten Aufheizens ist. Bei hohen Temperaturen schließen sich die neuen Messwerte im Rahmen der Messgenauigkeit analog zu den Ergebnissen aus Kapitel 6.1 
stetig an die vorigen Werte an. Somit kann auch hier ein Masseverlust ausgeschlossen werden, und stattdessen eine durch die Auslagerung induzierte Zunahme des Speichermoduls für die geringe Frequenzverschiebung bei niedrigen Temperaturen verantwortlich gemacht werden. Die Frequenzverschiebung nimmt für hohe Temperaturen über $400 \mathrm{~K}$ weiter zu, was analog zu den Ergebnissen aus Kapitel 6.1 auf einen überlagernden thermisch induzierten Prozess, der zur Härtung des Polymers führt, schließen lässt.

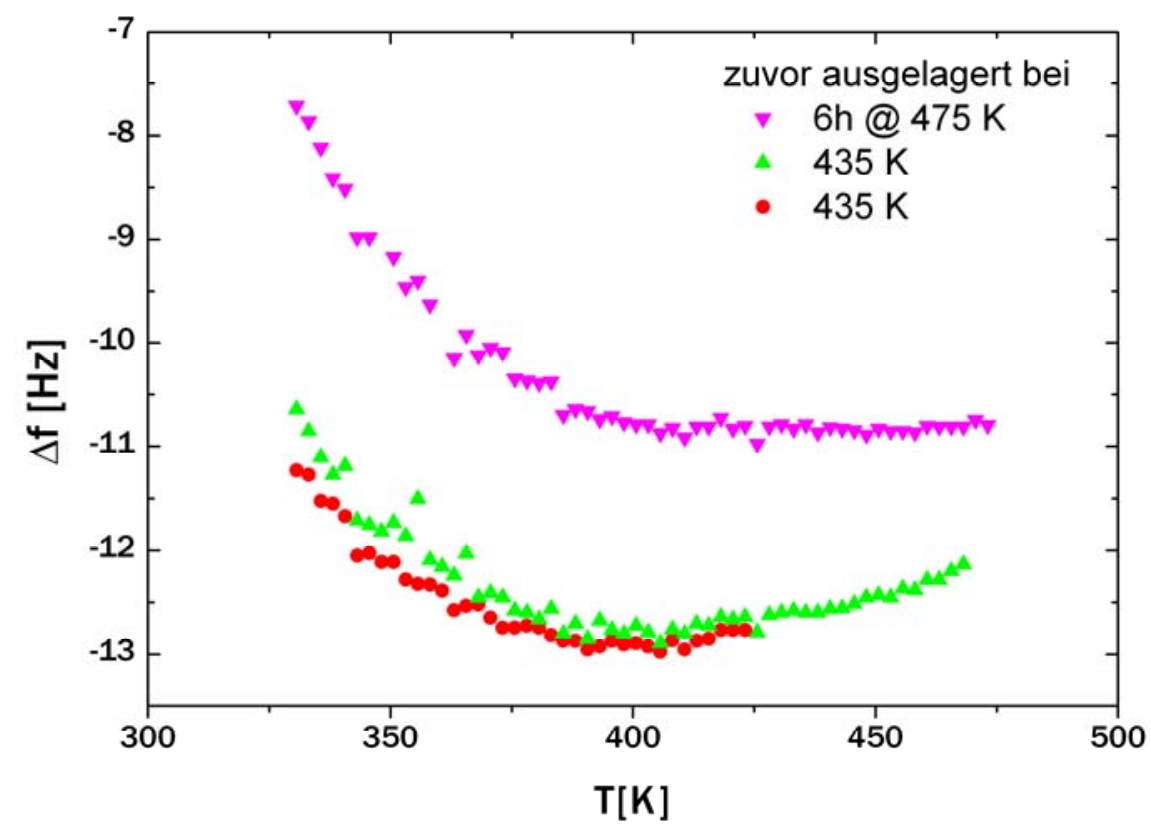

Abbildung 6.7: Gemessene temperaturabhängige Frequenzverschiebung des mit 10.000 Pulsen PEMA beschichteten Reeds bei unterschiedlicher Temperaturvorgeschichte. Die Messungen an den jeweils bei $435 \mathrm{~K}$ ausgelagerten Proben zeigen einen ähnlichen Verlauf, wobei die Kurve des zweiten Messdurchgangs leicht zu kleineren Beträgen verschoben ist. Das für 6 Stunden bei $475 \mathrm{~K}$ ausgelagerte System zeigt über den kompletten Temperaturbereich eine Verschiebung zu höheren Frequenzen, s. d. hier ein Masseverlust wahrscheinlich ist.

Zur Untersuchung der Langzeitstabilität wurde die Probe vor der folgenden Heizmessung für 6 Stunden bei $475 \mathrm{~K}$ ausgelagert. Die Resonanzfrequenz der so behandelten Probe ist über den gesamten Temperaturbereich stark angewachsen, die Frequenzverschiebung also verringert (magenta). Entgegengesetzt zu den früheren Messungen treffen sich die aufeinanderfolgenden Messungen auch bei hohen Temperaturen nicht mehr. Dies lässt den Schluss zu, dass für sehr lange Auslagerungszeiten tatsächlich Masseverlust auftreten kann. Ein Vergleich mit der Resonanzfrequenz des leeren Substrats liefert eine verbleibende Schichtdicke von 2,36 $\mu \mathrm{m}$, also einen Verlust von $15 \%$. Das bei den früheren Messungen beobachtete Ansteigen der Kurve ist hier nicht zu erkennen, stattdessen verläuft die Frequenzverschiebung ab etwa $400 \mathrm{~K}$ konstant. 


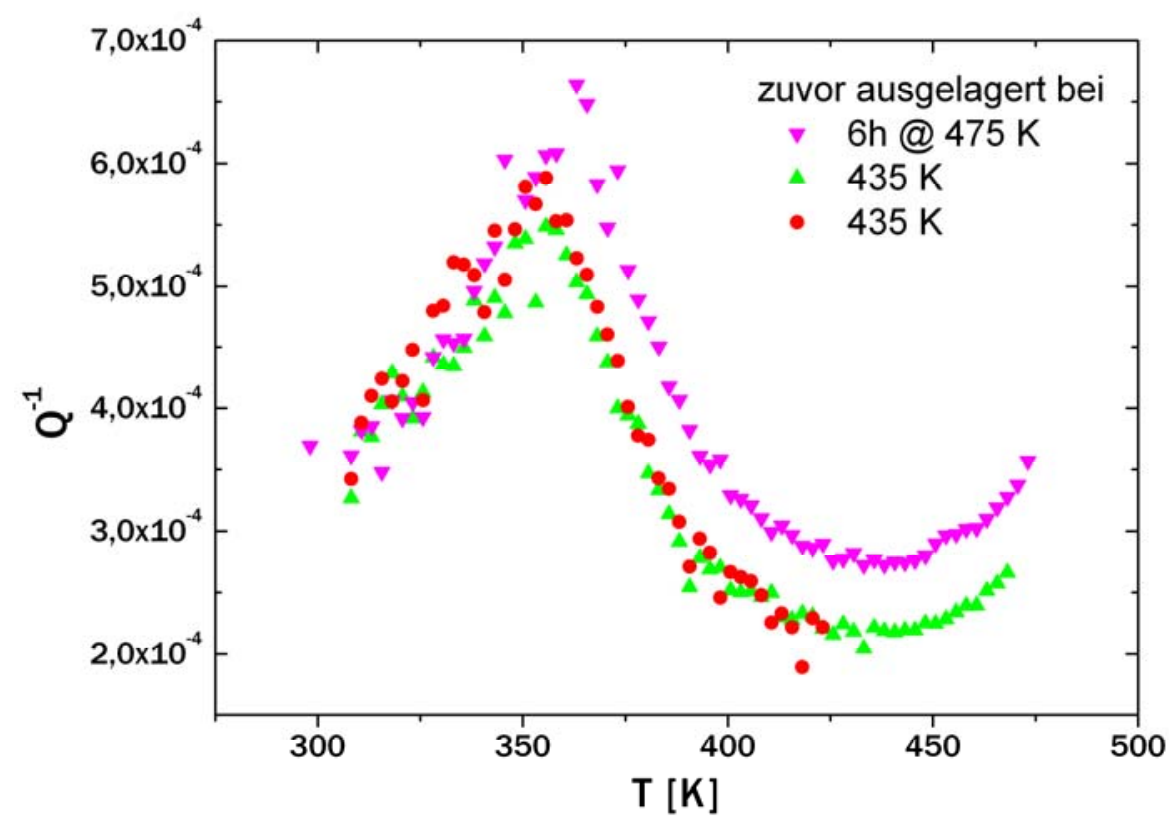

Abbildung 6.8: Temperaturabhängige Güte des mit 10.000 Pulsen PEMA beschichteten Reeds bei unterschiedlicher Temperaturvorgeschichte. Während die Messungen der bei $435 \mathrm{~K}$ ausgelagerten Probe im Rahmen der Messgenauigkeit übereinstimmen, weist die bei $475 \mathrm{~K}$ ausgelagerte Probe eine erhöhte Dämpfung auf.

Abbildung 6.8 zeigt die zugehörige temperaturabhängige Dämpfung für die bei hohen Temperaturen ausgelagerte Probe. Bis zur höchsten Auslagerungstemperatur von $435 \mathrm{~K}$ ist die Dämpfung bei zweifachem Durchlauf des Messprogramms reversibel. Bei den erstmals erreichten höheren Temperaturen (grüne Kurve) steigt die Dämpfung ab $435 \mathrm{~K}$ wieder an. Die nach der Langzeit-Auslagerung bei $475 \mathrm{~K}$ aufgenommene Dämpfung läuft trotz des oben diskutierten Masseverlusts ab etwa $360 \mathrm{~K}$ oberhalb der der vorigen Messung, was auf eine erhöhte Energiedissipation im ausgelagerten Film schließen lässt.

Aus den gemessenen Werten der Frequenzverschiebung und der Dämpfung kann der komplexe Elastizitätsmodul bestimmt werden. Die ermittelten Werte sind in Abbildung 6.9 für den reproduzierbar zugänglichen Temperaturbereich ab $330 \mathrm{~K}$ dargestellt.

In die Auswertung geht die Annahme ein, dass oberhalb von $T_{\mathrm{g}}$ eine Temperatur existiert, bei der der Speichermodul in der Schicht vollständig verschwindet. Gemäß Formel 4.31 und Formel 4.32 hängt der Speichermodul direkt von der Differenz der Quadrate der Resonanzfrequenzen von beschichtetem und unbeschichtetem Substrat ab. Bereits für die bei $435 \mathrm{~K}$ ausgelagerten Proben zeigt die Abnahme des Betrags der Frequenzverschiebung für Temperaturen über $400 \mathrm{~K}$ in Abbildung 6.7, dass die bisherige Annahme [16], diese Temperatur entspreche jeweils der höchsten betrachteten Temperatur $T_{\max }$, falsch sein muss. So zeigt der Be- 
trag der Frequenzverschiebung bei $400 \mathrm{~K}$ ein Maximum, was wiederum einem Minimum im Speichermodul entspricht. Dieses wäre zwangsläufig kleiner als Null, weil $E^{\prime}\left(T_{\max }\right) \equiv 0>E^{\prime}(400 \mathrm{~K})$, was physikalisch nicht sinnvoll ist. Stattdessen scheint es richtig, nicht die maximale Temperatur als Nullpunkt zu verwenden, sondern die Temperatur der maximalen Frequenzverschiebung. Gegen die Verwendung der maximalen Temperatur als Referenztemperatur spricht auch die Tatsache, dass die rote und die grüne Kurve bis $435 \mathrm{~K}$ einen sehr ähnlichen Verlauf zeigen, was aufgrund der identischen vorigen Auslagerungstemperatur und des somit identischen Relaxationszustands auch zu erwarten ist. Somit sollten die Speichermoduln bis zu $435 \mathrm{~K}$ auch identisch sein, was durch $E^{\prime}\left(T_{\max }\right) \equiv 0$ nicht gegeben wäre.

Für alle drei in Abbildung 6.9 gezeigten Kurven wurde somit $E^{\prime}(410 \mathrm{~K}) \equiv 0$ festgesetzt. Diese Annahme scheint zunächst willkürlich. Dennoch liefern die so bestimmten Ergebnisse sinnvolle Werte.
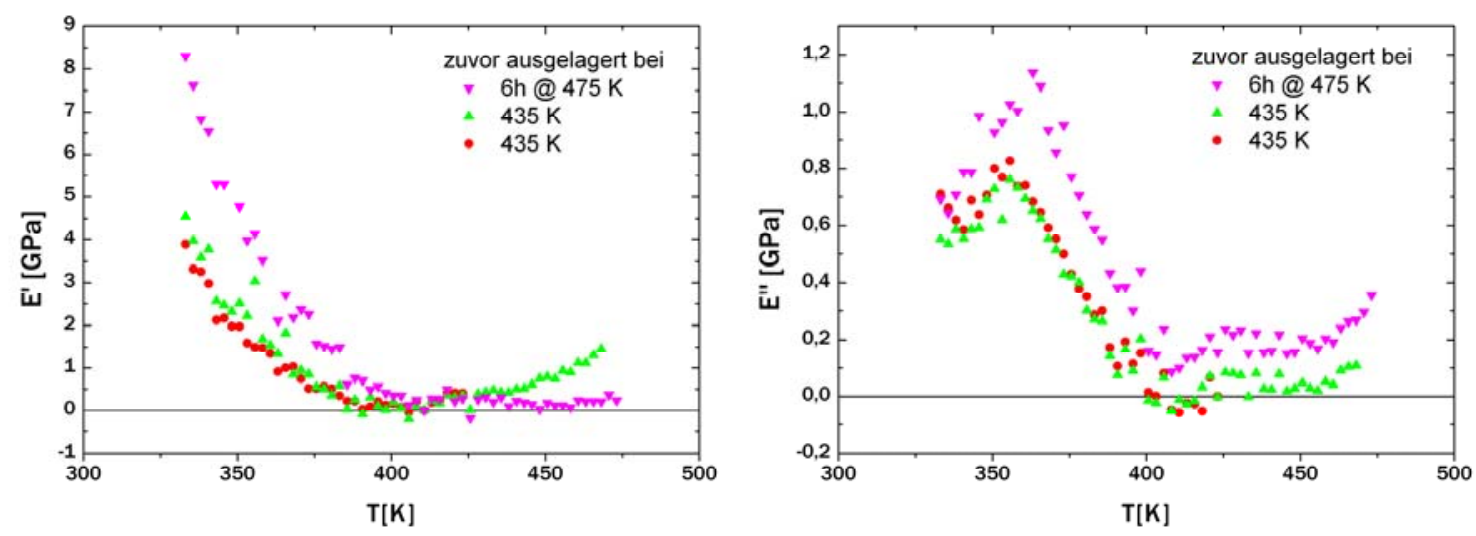

Abbildung 6.9: Speicher- (links) und Verlustmodul (rechts) einer mit 5.000 Pulsen hergestellten PEMA-Schicht, die zuvor bei $475 \mathrm{~K}$ ausgelagert wurde. Die langfristige Auslagerung bei $475 \mathrm{~K}$ führt zu Masseverlust von etwa 15\%. Für die so behandelte Probe verschwindet der Speichermodul für hohe Temperaturen vollständig.

Im Temperaturbereich unterhalb des Glasübergangs fehlen die Angaben des Speichermoduls, da hier, wie oben beschrieben, keine aussagekräftigen Messwerte vorliegen. Somit ist nur ein Abfall von $E^{\varsigma}$ zu erkennen. Für die bei $435 \mathrm{~K}$ ausgelagerten Proben knickt die Kurve bei etwa $375 \mathrm{~K}$ ab und steigt danach wieder an. Die Kurve der Langzeit-ausgelagerten Probe zeigt anfangs einen ähnlichen Abfall, allerdings mit etwa doppelt so großen absoluten Werten. Nach dem Abknicken folgt hier kein erneuter Anstieg, sondern der Speichermodul bleibt im Rahmen der Messgenauigkeit ab $410 \mathrm{~K}$ konstant bei Null. Die Auslagerung führt hier also neben dem Masseverlust zu einer erhöhten Härte des Materials bei geringen Temperaturen. Für hohe Temperaturen hingegen verschwindet der elastische Beitrag der Schicht für diese Probe. 
Der Verlustmodul zeigt für die bei $435 \mathrm{~K}$ ausgelagerten Proben nach anfänglicher Streuung der Messwerte ein Maximum mit $900 \mathrm{MPa}$ bei $355 \mathrm{~K}$ und dann einen Abfall bis auf Null. Der Modul der für 6 Stunden ausgelagerten Probe zeigt einen ähnlichen Verlauf, liegt aber im gesamten Temperaturbereich etwa $200 \mathrm{MPa}$ über den vorigen Werten und fällt somit auch für hohe Temperaturen nicht bis auf Null ab.

Der Vergleich der Ergebnisse, die mit dem DPO und dem PPXR erzielt wurden, bringt auf qualitativer Ebene viele Übereinstimmungen. Die Glasübergangsmaxima liegen im Rahmen der Messgenauigkeit im gleichen Temperaturbereich und die Form dieser Maxima ist sehr ähnlich, was auf ähnliche mikroskopische Dämpfungsprozesse schließen lässt. Auch der Einfluss von thermischer Auslagerung ist ähnlich und bewirkt eine Zunahme sowohl der jeweiligen Moduln als auch der Dämpfung. Dennoch zeichnen sich besonders im Temperaturbereich über $400 \mathrm{~K}$ Unterschiede ab. Während die mit PPXR bestimmte Dämpfung für die nicht Langzeit-ausgelagerte Probe wieder bis auf den Wert der Leermessung abfällt, liegt die Dämpfung im DPO bedeutend höher als die zugehörige Leermessung. Diese Unterschiede können verschiedene Gründe haben. Zum einen ist die Form der Anregung bei beiden Methoden grundsätzlich unterschiedlich. Auch die Art der beobachten Schwingung ist verschieden: Während beim DPO mit der AS2-Mode eine reine Torsionsmode verwendet wird, wird das PPXR zu Biegeschwingungen angeregt, s. d. mit dem DPO das Schermodul, mit dem PPXR das Elastizitätsmodul der Schicht abgefragt wird. Nicht zuletzt bleibt der Unterschied in der Güte der Schwingung der leeren Oszillatoren und damit in der Empfindlichkeit der beiden Methoden.

Zur genaueren Klärung der Unterschiede werden im Folgenden Untersuchungen vorgestellt, bei denen externe Einflüsse ausgeschlossen werden können. Die Unterschiede zwischen den Messungen beruhen nur auf der unterschiedlichen Frequenz sowie der Art der verwendeten Schwingung.

\subsection{Kombinierte Messungen (All-Mode-DPO)}

Mit dem Aufbau der in Kapitel 4.1.3 beschriebenen Anlage im Rahmen dieser Arbeit steht nun eine Methode zur Verfügung, mit der die qualitativen Unterschiede im Dämpfungsverlauf zwischen PPXR- und DPO-Messungen eingehend untersucht werden können. Hier sind die Stärken der beiden etablierten Methoden vereint. Zum einen ist das die Möglichkeit, die Mes- 
sungen in-situ durchzuführen, was zukünftig die Bearbeitung gänzlich neuer Fragestellungen, wie z. B. der Untersuchung der mechanischen Eigenschaften während des Schichtwachstums, erlaubt. Andererseits besteht die Möglichkeit, Messungen bei vielen unterschiedlichen Schwingungsmoden durchzuführen. Dadurch kann zwischen Torsions- und Biegeschwingung variiert werden, und es eröffnen sich die zusätzlichen Messfrequenzen zwischen 200 und 6.000 Hz. Die Messung der unterschiedlichen Schwingungsmoden erfolgt am identischen DPO und an ein und derselben Schicht, sowie in kurzer zeitlicher Abfolge, wodurch der Einfluss unterschiedlicher Substrate oder Schichtherstellungs- oder anderer Umgebungsparameter ausgeschlossen werden kann. Außerdem steht beim DPO mit der AS2-Mode eine Mode besonders hoher Güte zur Verfügung, die es ermöglicht, minimale Effekte zu untersuchen.

\subsubsection{Schwingungsverhalten des unbeschichteten DPOs}

Abbildung 6.10 und Abbildung 6.11 zeigen den Frequenz- und Dämpfungsverlauf eines unbeschichteten Doppelpaddeloszillators für dessen Torsions- bzw. Biegeschwingungsmoden im Temperaturbereich zwischen 235 und 500 K. Die vollen Symbole stellen dabei, wie auch in der Darstellung aller weiteren Messungen, die Daten der Heizmessung und die leeren Symbole die Daten der darauf folgenden Kühlmessung dar.

Die Frequenz sinkt für alle Moden wie erwartet mit steigender Temperatur und der Verlauf von Heiz- und Kühlmessung liegt für die antisymmetrischen Torsionsmoden gut übereinander, was auf eine sehr gute thermische Reproduzierbarkeit schließen lässt. Bei den weiteren Schwingungen zeigt sich eine Heizrichtungsabhängigkeit, die Kühlkurven zeigen im mittleren Temperaturbereich höhere Frequenzen als die jeweiligen Heizkurven. Dieser Effekt ist teilweise sehr stark. So liegen die beiden Kurven z. B. bei der CL2-Mode um bis zu $62 \mathrm{~K}$ auseinander (bei $f=1595 \mathrm{~Hz}$ mit zugehörigen Temperaturen $T_{\text {Heiz }}=400 \mathrm{~K}$ und $T_{\text {Kühl }}=462 \mathrm{~K}$ ). Eine solch starke Abweichung ist, auch in Hinblick auf die gute Reproduzierbarkeit bei den Torsionsmoden und die Ergebnisse der FEM-Rechnungen in Kapitel 4.1.3.3, sicherlich auszuschließen. Wichtiger scheint der Einfluss des Temperaturgradienten im Bein des DPOs zu sein, der, bedingt durch die schlechte Wärmeleitung über den kleinen Querschnitt auch nach langer Wartezeit auftreten kann. Dieser Gradient reagiert auf eine Umkehrung der Heizrichtung sensibler als die absolute Temperatur. Der unterschiedlich starke Einfluss auf die Biegeund Torsionsschwingungen lässt sich über den an der Schwingung beteiligten Bereich erklären. Während der Großteil der Verformung bei den antisymmetrischen Torsionsmoden im 
Hals, also sehr lokal und in einem thermisch homogenen Bereich, passiert, ist bei den Biegeschwingungen zu großen Teilen auch der Fuß des DPOs beteiligt. Der Gradient im tatsächlich schwingenden Bereich ist hier also bedeutend größer und somit auch sein Einfluss auf die Resonanzfrequenz. Nicht abschließend geklärt werden kann hier allerdings, warum der Gradient im Bein auch nach der Wartezeit vor der jeweiligen Messung noch einen solch starken Einfluss hat. Dabei ist anzumerken, dass eine so große Abweichung zwischen Heiz- und Kühlmessung nur bei diesem DPO aufgetreten ist.

Bei den Dämpfungskurven ist zusätzlich der theoretisch erwartete Verlauf für eine Dämpfung durch den thermoelastischen Effekt eingetragen. In diesem Modell führt die mechanische Oszillation im elastischen Material zu einem Temperaturgradienten zwischen den lokal komprimierten und den lokal aufgeweiteten Bereichen des Gitters [118]. Der induzierte Wärmefluss und die damit verbundene inelastische Streuung der thermisch erzeugten Phononen führen zur Energiedissipation und somit zur Gesamtdämpfung im Oszillator. Es gilt nach [119]:

$$
Q_{T . E .}^{-1}(T)=\rho \frac{E \alpha^{2} T}{c} \frac{\omega \tau}{1+\omega^{2} \tau^{2}}
$$

mit der thermischen Relaxationszeit

$$
\tau=\frac{t_{D P O}^{2} c}{\pi^{2} \kappa^{2}}
$$

Dabei beschreibt der modenabhängige Vorfaktor $\rho$ den Anteil der Biegeschwingungsenergie an der Gesamtschwingungsenergie. Dieser wurde für die AS2-Mode über Finite Elemente Modellierungen zu $\rho=0,0755$ bestimmt [119]. Dieser Wert wurde im Folgenden auch für die weiteren Torsionsmoden verwendet. Für die Clapping Moden ist $\rho$ natürlich größer und wurde hier fest auf 0,75 gesetzt.

Alle weiteren Parameter sind durch die Eigenschaften des Oszillators bestimmt: $E=169 \mathrm{GPa}$ ist der Elastizitätsmodul, $t_{\mathrm{DPO}}=300 \mu \mathrm{m}$ die Dicke des Oszillators und $\omega=2 \pi f$ ist die Winkelgeschwindigkeit der Schwingung. Die weiteren temperaturabhängigen Materialeigenschaftenthermischer Ausdehnungskoeffizient $\alpha$, spezifische Wärme pro Volumen $c$ und thermische Leitfähigkeit $\kappa$-wurden [120] entnommen. 

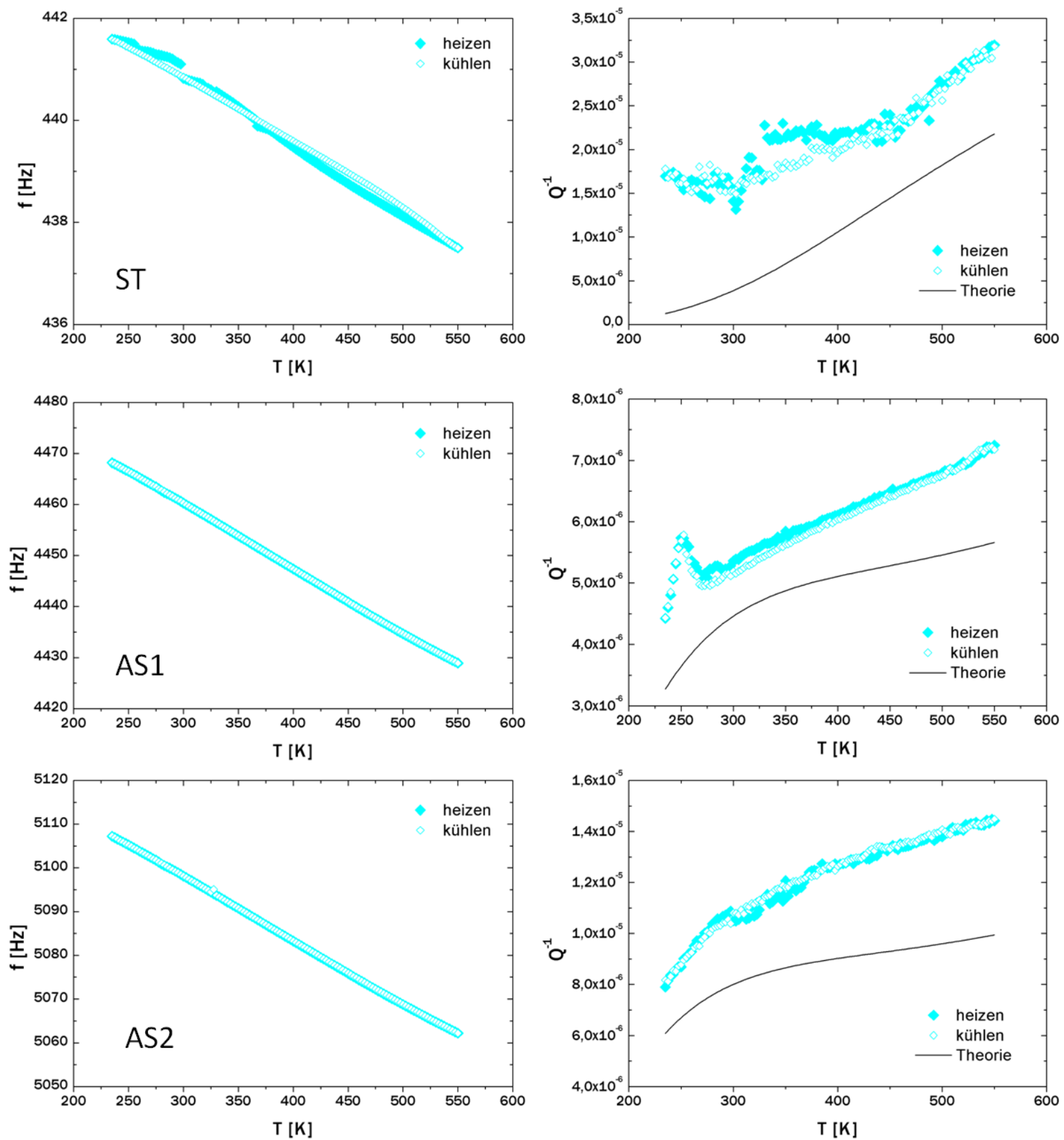

Abbildung 6.10: Temperaturabhängiger Frequenz- und Dämpfungsverlauf der Leermessungen für die drei verschiedenen Torsionsmoden. Die eingezeichneten theoretischen Verläufe beschreiben die Dämpfung durch den thermoelastischen Effekt, der die Temperaturabhängigkeit der Dämpfung dominiert.

Die experimentell bestimmten Werte liegen meist über den theoretisch erwarteten, was auf weitere Dämpfungsmechanismen schließen lässt, die z. B. auf Defekte im Festkörper [121], Oberflächeneffekte [121, 122], Verluste in der Klemmung [95, 122] oder Kontamination des DPOs zurückzuführen sind. Die Temperaturabhängigkeit wird jedoch sehr gut durch den thermoelastischen Effekt beschrieben. 

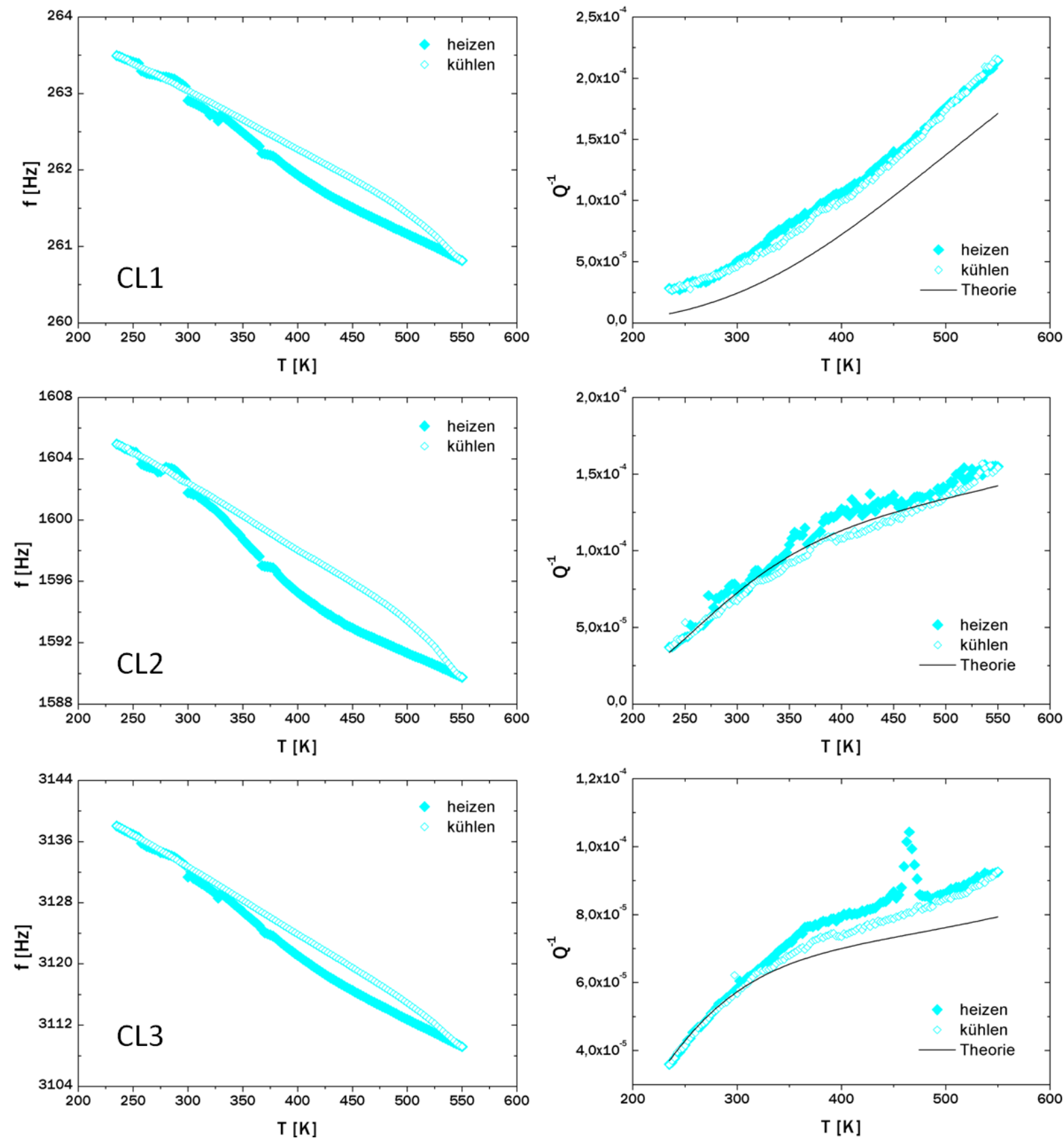

Abbildung 6.11: Temperaturabhängiger Frequenz- und Dämpfungsverlauf der Leermessungen für die Biegeschwingungen. Die eingezeichneten theoretischen Verläufe beschreiben die Dämpfung durch den thermoelastischen Effekt, der die Temperaturabhängigkeit der Dämpfung dominiert.

Sowohl bei der AS1-, als auch bei der CL3-Mode ist im Dämpfungsverlauf ein reproduzierbares Maximum zu sehen. Solche Maxima treten bei verschiedenen DPOs bei unterschiedlichen Temperaturen und unterschiedlich stark auf. Die Ursache dieser zusätzlichen Dämpfung ist noch nicht vollständig geklärt, möglich wäre zum Einen ein temperaturabhängiges Lösen des DPOs in der Klemmung aufgrund der unterschiedlichen Ausdehnungskoeffizienten von Halter und DPO. Andererseits könnten Verunreinigungen im DPO einen Snoek-Effekt, also das resonante Springen von Fremdatomen zwischen interstitiellen Gitterplätzen, hervorrufen, wodurch 
die recht scharfen Maxima zu erklären wären [83]. Auch die in Kapitel 4.1.1 dargestellten, durch das Laserschneiden der DPOs eingebrachten Defekte an den Grenzflächen könnten als Ursache in Frage kommen. Gegen diese Thesen spricht die Tatsache, dass die Temperatur, bei der ein Maximum auftritt, bei späterer Wiederholung nicht vollständig reproduzierbar ist. Somit muss hier die tatsächliche Ursache unklar bleiben. Die Störungen sind aber klar als solche zu identifizieren und können somit nicht zu einer Verfälschung der Messergebnisse führen.

\subsubsection{Speicher- und Verlustmodul}

Zur Bestimmung der mechanischen Eigenschaften einer PEMA-Schicht wurde der oben charakterisierte DPO nach der Leermessung mit 5.000 Laserpulsen PEMA beschichtet und erneut vermessen. Um auch hier Relaxationsprozesse des laserdeponierten Materials beobachten zu können, wurde auf eine Auslagerung vor der Messung verzichtet.

Zunächst soll im Vergleich zu Kapitel 6.1 der Einfluss der Schicht auf das Schwingungsverhalten der AS2-Mode untersucht werden. Abbildung 6.12 zeigt die relative Änderung der AS2-Resonanzfrequenz für drei Temperaturzyklen mit unterschiedlicher Endtemperatur. Die vollen Symbole stellen wieder die Heiz-, die leeren Symbole die Kühlmessungen dar. Für die schwarze Neukurve ist zunächst ein horizontaler Verlauf zu beobachten, der ab $350 \mathrm{~K}$ zu einer geringen Frequenzdifferenz abknickt. Beim Abkühlen wird dieser Verlauf nicht reproduziert, vielmehr verläuft die Frequenzdifferenz bei geringeren Werten zurück. Die nächste Heizkurve (volle rote Punkte) liegt bis zur vorigen Endtemperatur identisch auf der letzten Kühlkurve und setzt diese dann stetig fort. Die Daten der folgenden Kühlmessung sind wieder zu kleinerer Frequenzverschiebung versetzt. Diese Werte werden sowohl von der Heiz- als auch der Kühlkurve der letzten Messung (grüne Dreiecke) reproduziert.

Der Verlauf der Frequenzverschiebung ist also von der vorherigen Auslagerungstemperatur abhängig. Ist diese gleich, werden die Daten reproduziert. Gemäß Formel 4.19 ist diese Frequenzverschiebung direkt mit dem Schermodul der Schicht verknüpft, die stetige Abnahme der Frequenzverschiebung ist also mit einer Zunahme des Schermoduls verbunden, wie bereits in Kapitel 6.1 diskutiert. Dieser Prozess überlagert die durch den Glasübergang bestimmte Abnahme des Schermoduls, die in Abbildung 6.14 quantitativ diskutiert wird. 


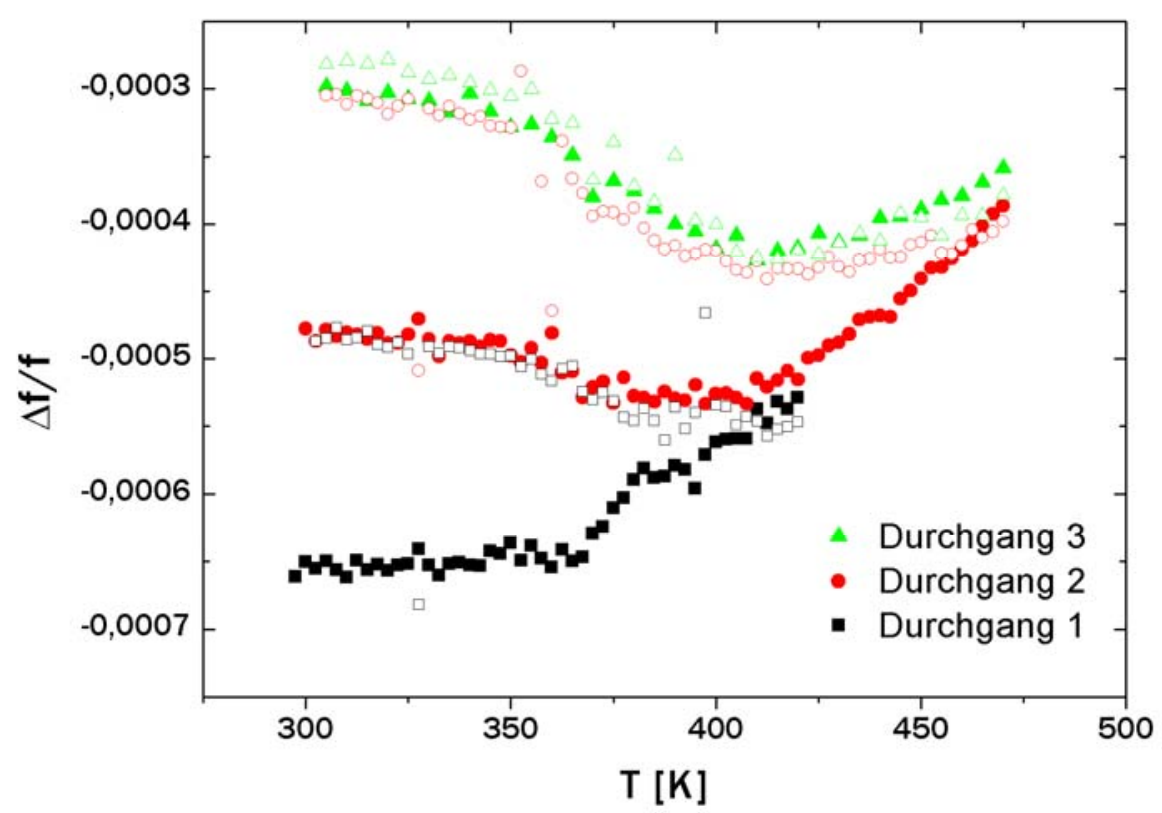

Abbildung 6.12: Relative Frequenzänderung der AS2-Resonanzfrequenz durch eine mit 5.000 Pulsen laserdeponierte PEMA-Schicht mit unterschiedlicher thermischer Vorgeschichte. Die vollen schwarzen Quadrate beschreiben den ersten Durchgang nach der Deposition. Die leeren Quadrate zeigen die Daten der darauf folgenden Kühlmessung. Analog zeigen die roten und grünen Symbole die Werte für den zweiten und dritten Messzyklus.

Abbildung 6.13 zeigt den zugehörigen Verlauf der Dämpfungsdifferenz zwischen beschichtetem und leerem DPO. Die Temperaturabhängigkeit ähnelt dem in Kapitel 6.1 gezeigten Verhalten, zeigt jedoch auch Unterschiede. Zunächst zeigt die Neukurve im Vergleich mit allen folgenden Heiz- und Kühlkurven eine Verschiebung des Maximums um etwa $10 \mathrm{~K}$. Diese lässt sich wieder durch überlagernde thermisch induzierte Relaxationsprozesse während des Aufheizens erklären. Wie in Kapitel 6.1 dargelegt, setzen diese erst ab einer Temperatur von $375 \mathrm{~K}$ signifikant ein, s. d. das Maximum hier unterhalb dieser Temperatur unterdrückt wird. Das Maximum ist also nicht wirklich verschoben, sondern es fehlen lediglich die Beiträge unterhalb von $375 \mathrm{~K}$, was zur Deformation des Maximums und somit zur scheinbaren Verschiebung führt.

Die folgende Heizmessung reproduziert nicht, wie anhand der Ergebnisse aus den Frequenzmessungen erwartet werden könnte, die Daten der ersten Kühlmessung, sondern zeigt über den kompletten Temperaturbereich eine höhere Dämpfung. Dieses Verhalten kann hier nicht eindeutig erklärt werden, besonders da die weiteren Messungen wieder die bekannte Abhängigkeit der Dämpfung von der vorigen Auslagerungstemperatur zeigen. 


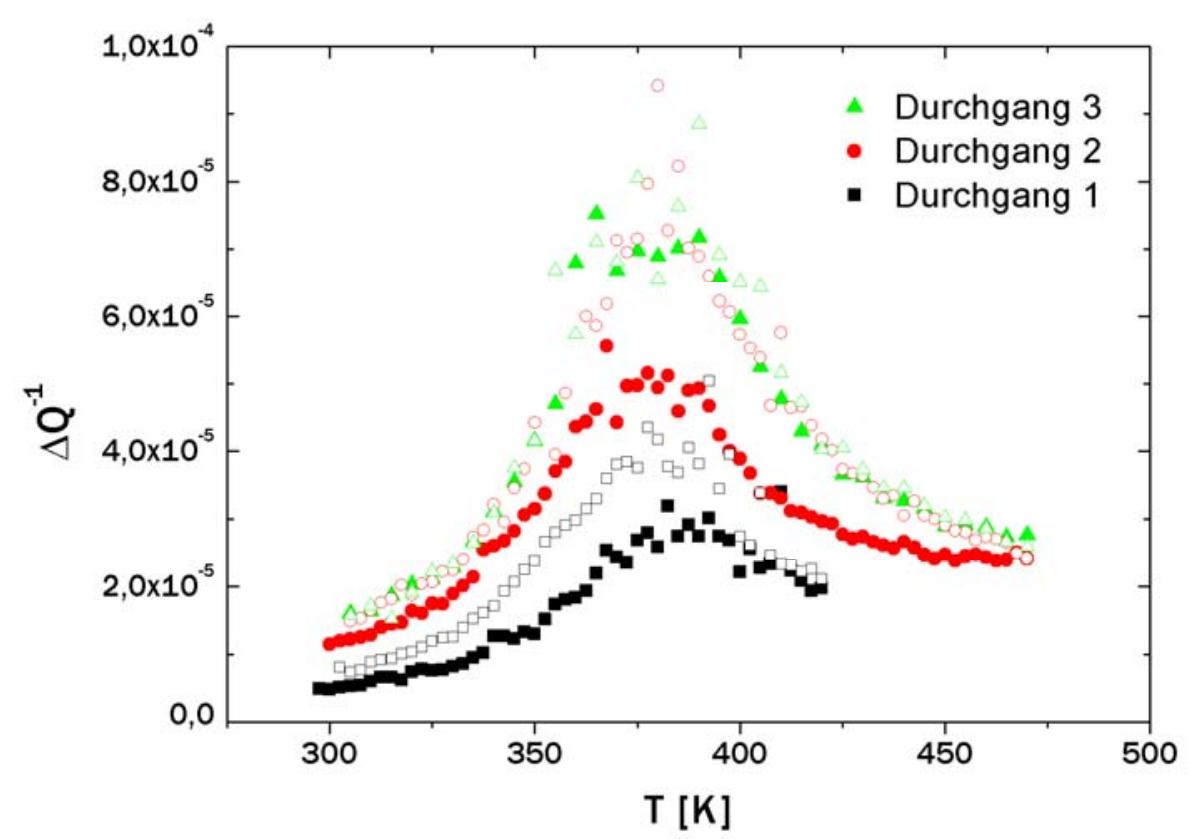

Abbildung 6.13: Differenz der inversen Qualitätsfaktoren von beschichtetem und unbeschichtetem DPO der AS2Mode an einer mit 5.000 Pulsen laserdeponierten PEMA-Schicht mit unterschiedlicher thermischer Vorgeschichte. Die vollen schwarzen Quadrate beschreiben den ersten Durchgang nach Deposition. Die leeren Quadrate zeigen die Daten der darauf folgenden Kühlmessung. Analog zeigen die roten und grünen Symbole die Werte für den zweiten und dritten Messzyklus.

Zur quantitativen Berechnung des Schermoduls ist nun gemäß Formel 4.20 die Schichtdicke erforderlich. Diese wurde anhand von der bekannten Depositionsrate zu $t_{\text {Film }}=1,4 \mu \mathrm{m}$ bestimmt. Abbildung 6.14 zeigt die so bestimmten Real- und Imaginärteile des Schermoduls abhängig von der vorigen Auslagerung. Zusätzlich eingetragen sind die Daten eines vierten Messdurchgangs, der nach dem dritten Durchgang bis $470 \mathrm{~K}$ durchgeführt wurde.

Wie auch schon bei der Betrachtung der Frequenzverschiebung sind beim Speichermodul zwei unterschiedliche Effekte zu erkennen. Das Erhitzen der Probe auf „neue“ Temperaturen, also Temperaturen, der die Probe zuvor noch nicht ausgesetzt war, führt ab $360 \mathrm{~K} \mathrm{zu}$ einer starken, monotonen und irreversiblen Erhöhung des Speichermoduls mit steigender Temperatur. Diesem Prozess überlagert ist mit dem Erweichen der Schicht der eigentliche Glasübergang. Dieser ist in allen Messungen ausschließlich der ersten Heizmessung klar zu erkennen, bei mittleren Auslagerungstemperaturen (Kühlmessung des ersten Durchgangs, folgende Heizmessung) zeigt sich klassisches Verhalten: Der Speichermodul zeigt drei charakteristische Bereiche: Bis $330 \mathrm{~K}$ nimmt $G^{\star}$ mit steigender Temperatur leicht linear ab, danach fällt er schneller, aber weiter linear ab, um schließlich in ein Plateau abzuknicken. Die Heiz- und Kühlmessungen liegen gut übereinander, was auf gute Reproduzierbarkeit schließen lässt. 
Die weitere Auslagerung in Durchgang 2, 3 und 4 führt zu zusätzlicher, irreversibler Härtung des Films. Parallel ist für die letzten beiden Durchgänge ein minimales Abdampfen nicht vollständig auszuschließen, da die Messdaten für den letzten Durchgang systematisch über denen des dritten Durchgangs liegen.

Aus dem Anfang des Maximums im Verlustmodul lässt sich die Glasübergangstemperatur zu $330 \mathrm{~K}$ ablesen und verifiziert damit den in Kapitel 6.1 bestimmten Wert. Die Temperatur des Maximums in der Auftragung von $G^{\text {“ }}$ entspricht gemäß den Kramers-Kronig-Relationen $[123,124]$ der Temperatur des Wendepunkts der Kurve des Speichermoduls. Die Temperatur des folgenden Wendepunkts in $G^{\text {“ }}$ ist identisch mit der Temperatur des zweiten Knickpunkts in $G^{6}$.

Während der Speichermodul mit steigender Auslagerungstemperatur ansteigt, führt die thermisch induzierte Relaxation auch zu einer starken Zunahme des Verlustmoduls, was auf eine erhöhte Effizienz der Energiedissipation im Material zurückzuführen ist.
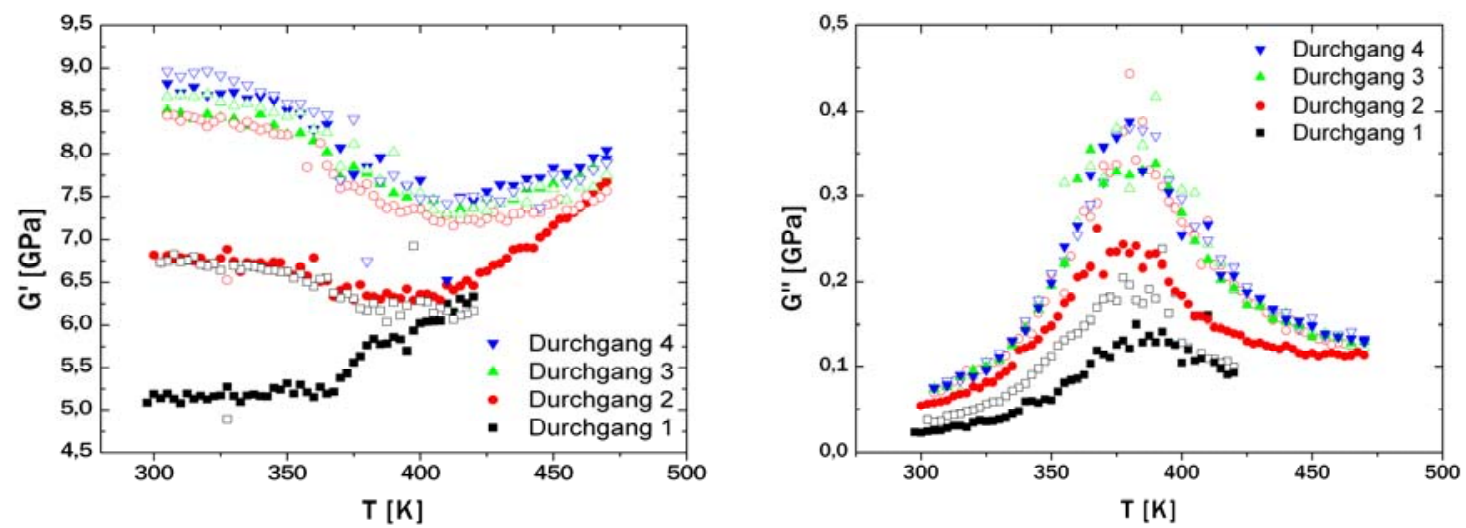

Abbildung 6.14: Temperaturabhängiger Schermodul der laserdeponierten PEMA-Schicht im ausgelagerten Zustand für Heiz- und Kühlmessung. Links ist der Speichermodul, rechts der Verlustmodul abgebildet. Als Schichtdicke für die Berechnung der Module wurde 1,4 $\mu \mathrm{m}$ verwendet.

\subsubsection{Frequenz- und Modenabhängigkeit}

Beginnend mit der ersten Antisymmetrischen Torsionsmode AS1 sollen im Folgenden die Ergebnisse, die über die mechanische Spektroskopie mit den weiteren Moden des DPOs gewonnen wurden, dargestellt werden.

Abbildung 6.15 zeigt die relative Verschiebung der AS1-Resonanzfrequenz zwischen beschichtetem und unbeschichtetem DPO sowie den zugehörigen Dämpfungsverlauf für die 
ersten drei Messdurchgänge. Der Verlauf entspricht bis auf leichte Änderungen bei den absoluten Werten dem bereits aus der Untersuchung der AS2-Mode bekannten Verhalten. Auch die AS1-Mode zeichnet sich durch eine hohe Empfindlichkeit aus und die gewonnenen Werte sind mit denen der AS2-Mode durchaus vergleichbar.
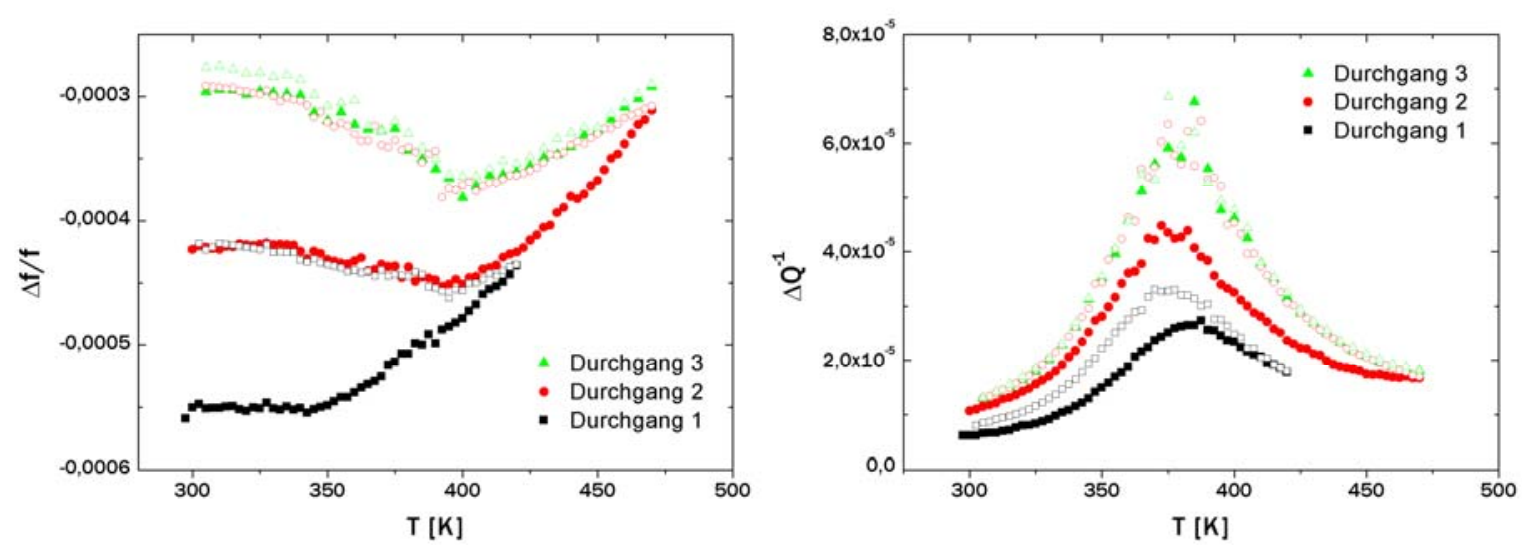

Abbildung 6.15: Relative Frequenzverschiebung und Differenz des inversen Qualitätsfaktors für die AS1-Mode bei etwa $4.450 \mathrm{kHz}$.

Signifikante Unterschiede fallen erst beim Vergleich mit den Daten, die über die Biegeschwingungen aufgenommen wurden, auf. Hier sind, wie auch schon bei den Leermessungen, in Abbildung 6.11 starke Unterschiede zwischen den Heiz- und den Kühlkurven zu erkennen.

Abbildung 6.16 zeigt die um die Leermessungen korrigierten Werte von Resonanzfrequenz und Dämpfung für die schnellste gemessene Biegeschwingung, die CL3-Mode bei etwa 3.120 Hz. Da die Subtraktion des in Kapitel 6.3.1 beschriebenen hysteretischen Frequenzverlaufs der Leermessungen von diesen Daten zu unphysikalischen Sprüngen an den Temperaturumkehrpunkten führen würde, wurde als Referenzkurve nicht jeweils die Heiz- bzw. Kühlkurve der Leermessung verwendet, sondern der Mittelwert aus diesen beiden Messungen. Hier nicht gezeigte Auftragungen, bei denen für die Heiz- und Kühlkurven des beschichteten Systems jeweils nur die Kühl-Leermessungen bzw. nur die Heiz-Leermessungen subtrahiert wurden, zeigen, dass der grundsätzliche Kurvenverlauf in beiden Fällen sehr ähnlich ist. Somit ist die Annahme des Mittelwerts als Referenzkurve hier für die qualitative Analyse gerechtfertigt.

Beim ersten Heizen steigt $\Delta f l f$ zunächst an, zeigt bei $320 \mathrm{~K}$ ein Maximum und fällt dann weiter ab. Die folgende Messung reproduziert beim Abkühlen bis etwa $375 \mathrm{~K}$ zunächst die Daten der Heizmessung, weist ab dieser Temperatur aber eine stärkere Frequenzverschiebung auf 
und zeigt kein Maximum. Bei Raumtemperatur ist der Betrag der Frequenzverschiebung nach dem Abkühlen größer als zu Beginn der Heizmessung.

Bei der nächsten Heizmessung (rote Kurve) ist die Position des Maximums zu höheren Temperaturen verschoben, s. d. zusätzlich ein Plateau bei kleinen Temperaturen bis $310 \mathrm{~K}$ erkennbar ist. Außerdem ist das Maximum breiter ausgeprägt. Bei $420 \mathrm{~K}$ ist ein Knickpunkt zu erkennen, nach dem die Frequenzverschiebung nahezu konstant bleibt. Die Daten der folgenden Kühlmessung reproduzieren nicht die der Heizmessung, sondern verlaufen oberhalb dieser parallel zur ersten Kühlmessung und zeigen wie diese kein Maximum. Die Heizmessung des dritten Zyklus reproduziert im Wesentlichen das Verhalten des vorigen, ist aber wieder zu geringeren Beträgen verschoben. Auch die folgende Kühlmessung läuft parallel zur vorigen, ist aber nur noch schwach verschoben, was auf eine nur noch schwache Veränderung im Material zurückzuführen ist.
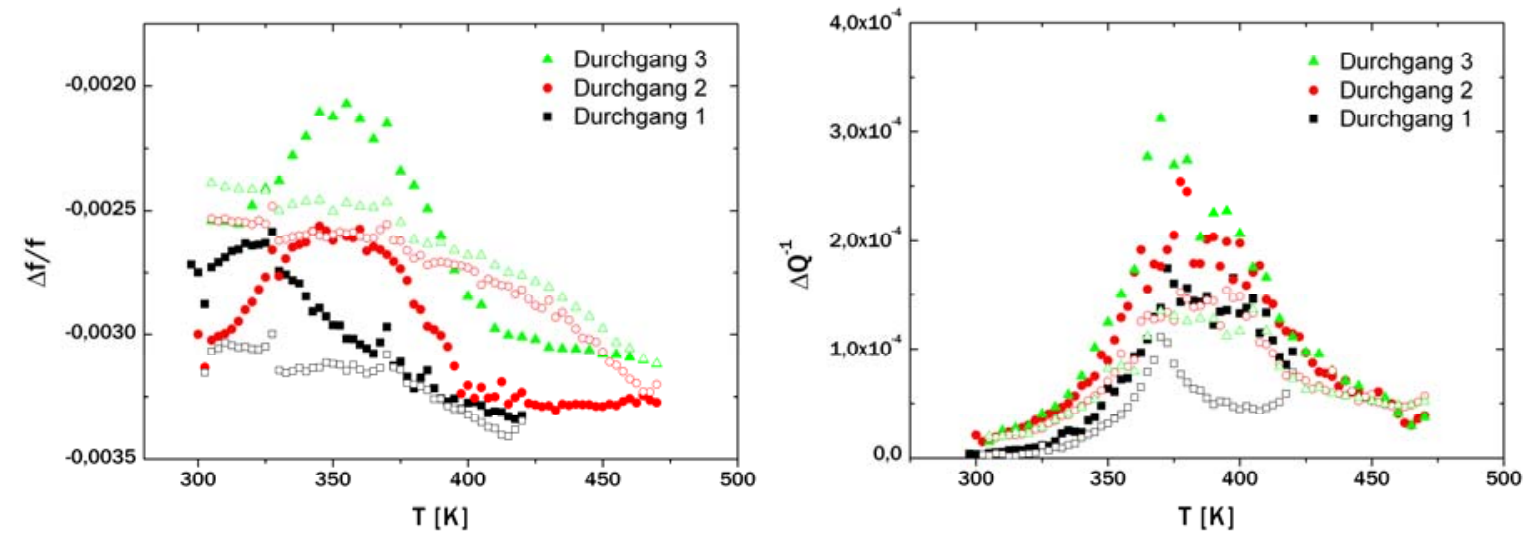

Abbildung 6.16: Relative Frequenzverschiebung und Differenz des inversen Qualitätsfaktors für die CL3-Mode bei 3.120 Hz. Sowohl in der Frequenz, als auch in der Dämpfung sind signifikante Unterschiede zwischen der Heiz- und der Kühlmessung zu erkennen.

Zusammenfassend sind hier zwei Effekte zu unterscheiden, wobei der Verlauf der Neukurve vernachlässigt wird, da hier weitere überlagernde Prozesse auftreten können, die eine systematische Beschreibung erschweren:

1. Innerhalb eines Messdurchgangs sind starke Unterschiede zwischen den Heiz- und den Kühlmessungen zu erkennen, die sich besonders im Auftreten eines Maximums zwischen 310 und $390 \mathrm{~K}$ in den Heizkurven manifestieren. So entsprechen weder die Daten der Heiz- noch die der Kühlkurven dem anhand der Ergebnisse der Messungen an den AS-Moden erwarteten Verlauf. Statt des Erweichens der Schicht am Glasübergang lassen die während des Heizens aufgenommenen Daten zwischen 300 und 325 K zunächst auf eine starke Zunahme des Spei- 
chermoduls schließen. Ab $370 \mathrm{~K}$ nimmt dieser dann bis $400 \mathrm{~K}$ wieder stark und oberhalb von $400 \mathrm{~K}$ nur noch leicht ab. Auch die Daten der Kühlmessung zeigen keinen eindeutigen Erweichungspunkt, sondern eine kontinuierliche Abnahme des Betrags der Frequenzverschiebung mit sinkender Temperatur, also eine Zunahme des Speichermoduls.

2. Die Auslagerung während der Messdurchgänge führt zu einer geringeren Frequenzverschiebung über den gesamten Temperaturbereich, im oben diskutierten Bild also zu einem höheren Speichermodul. Auch bei den Kühlmessungen von Durchgang 2 und Durchgang 3 tritt eine kleine, aber signifikante Verschiebung zu geringeren Beträgen auf, obwohl die Auslagerungstemperatur nicht weiter erhöht wurde. Die thermisch induzierte Relaxation war hier also nach Durchgang 2 noch nicht vollständig abgeschlossen, sondern die Filmeigenschaften wurden durch das erneute Aufheizen weiter modifiziert.

In Abbildung 6.16 ist rechts der zugehörige Dämpfungsverlauf aufgetragen. Auch hier zeigen sich Unterschiede zwischen den Heiz- und Kühlmessungen. Entgegengesetzt zu den Ergebnissen der AS-Moden liegen alle Kühlkurven unterhalb der zugehörigen Heizkurven. Eine Besonderheit stellt dabei die erste Kühlkurve dar: Sie liegt im Vergleich zu den weiteren Zyklen nicht nur bei tieferen Werten von $Q^{-1}$, sondern zeigt auch ein sehr viel schmaleres Maximum bei etwa $370 \mathrm{~K}$. Da dieses Verhalten nicht reproduziert werden konnte, wird hier, wie auch schon bei der Beschreibung der zugehörigen Frequenzverschiebung, auf eine weitere Diskussion verzichtet. Der während der Heizmessungen aufgenommene Dämpfungsverlauf ähnelt dem aus den antisymmetrischen Torsionsmoden bestimmten. Allerdings sind die Spitzenwerte der jeweiligen Maxima zwischen 365 und $405 \mathrm{~K}$,abgeschnitten“, es zeigt sich also keine hohe Spitze, sondern ein breites Plateau. Auch ist das Anwachsen der Maximumsintensität mit steigender Auslagerungstemperatur nicht so stark ausgeprägt, wie bei den AS-Moden.

Die an der Symmetrischen Torsionsmode ST aufgenommenen Messwerte bestätigen die obigen Unterschiede zwischen den Heiz- und den Kühlmessungen. Die in Abbildung 6.17 links dargestellten Frequenzwerte, zeigen einen ähnlichen Verlauf wie die der CL3-Mode. Es fehlt hier allerdings das Plateau bei tiefen Temperaturen, die Frequenzen der Kühlkurven fallen hier über den gesamten Temperaturbereich mit vergleichbarer Steigung ab. Auch die Dämpfungsmessungen verlaufen in weiten Teilen analog zu den oben beschriebenen. In den Kühlkurven der Dämpfungsmessungen ist jedoch zusätzlich eine signifikante Verschiebung des Maximums zu geringeren Temperaturen zu erkennen. 

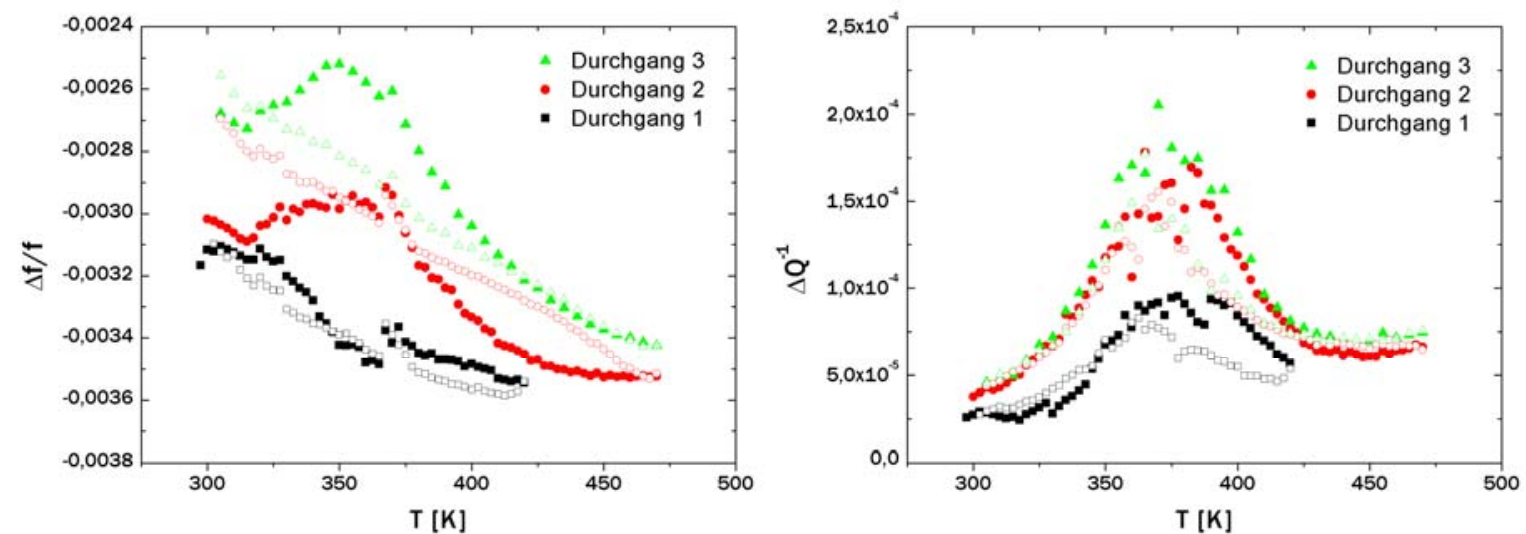

Abbildung 6.17: Relative Frequenzverschiebung und Differenz des inversen Qualitätsfaktors für die ST-Mode bei $440 \mathrm{~Hz}$.
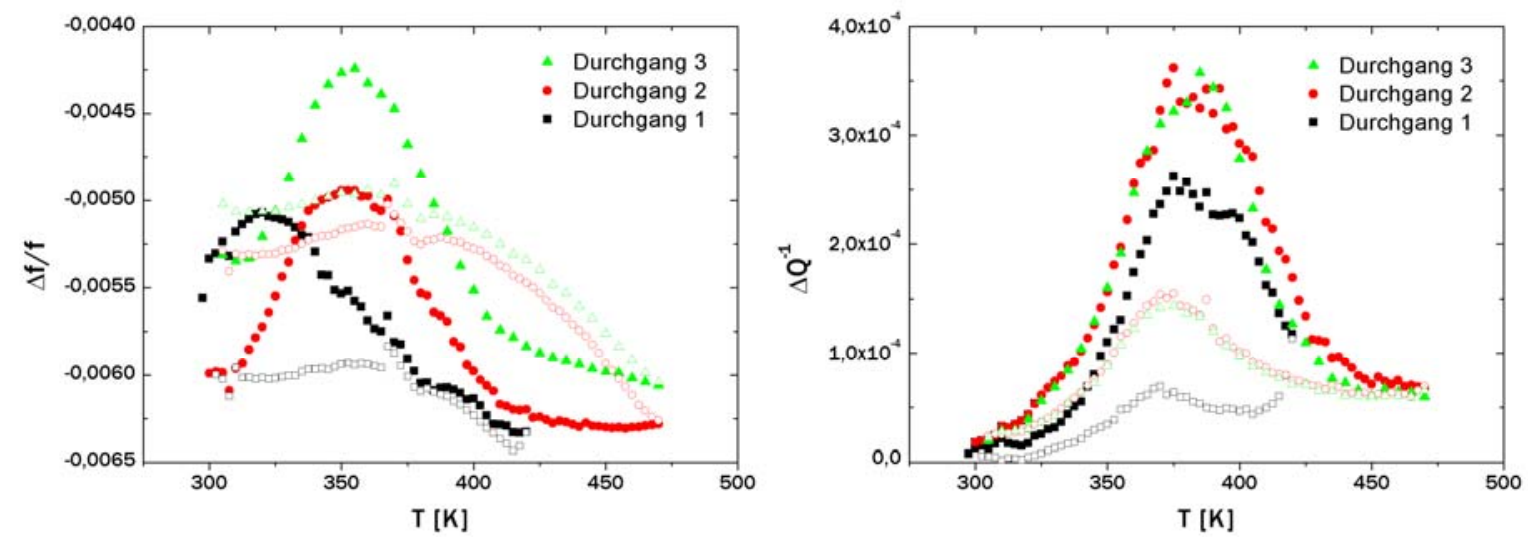

Abbildung 6.18: Relative Frequenzverschiebung und Differenz des inversen Qualitätsfaktors für die CL2-Mode bei $1.590 \mathrm{~Hz}$.
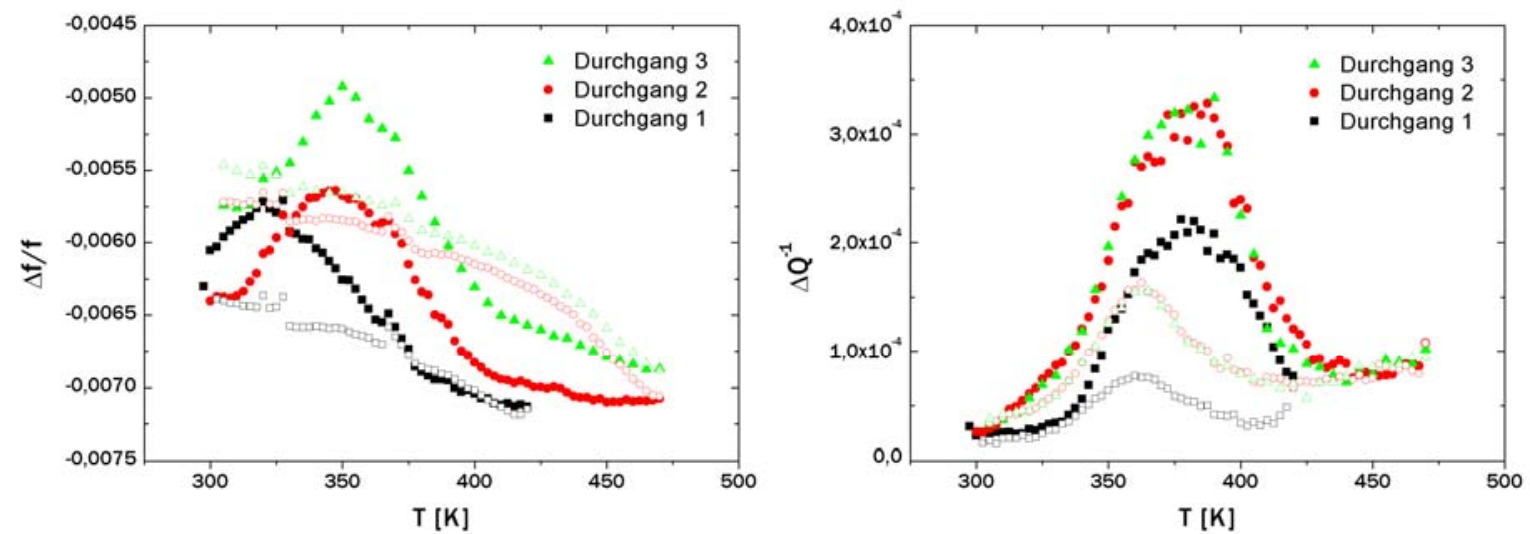

Abbildung 6.19: Relative Frequenzverschiebung und Differenz des inversen Qualitätsfaktors für die CL1-Mode bei $260 \mathrm{~Hz}$.

Auch die in Abbildung 6.18 und Abbildung 6.19 für die beiden weiteren Biegeschwingungsmoden gezeigten Graphen von Frequenzverschiebung und Dämpfungsdifferenz zeigen einen ähnlichen Verlauf wie die CL3-Mode und ST-Mode, haben aber ein besseres Signal-Rausch- 
Verhältnis, s. d. die Unterschiede zwischen Heiz- und Kühlmessungen hier präzise untersucht werden können. Insbesondere fällt auf, dass nicht nur die maximale Intensität der Dämpfung der Kühlmessung im Vergleich zu der der Heizmessung massiv zurückgegangen ist. Auch die Lage des Maximums ist zu geringeren Temperaturen verschoben. Der Grad der Abschwächung der Dämpfung während der Kühlmessung ist also auch von der anliegenden Temperatur abhängig.

Zusammenfassend können die Unterschiede zwischen den Heiz- und Kühlmessungen für alle Moden, an denen ein großer Bereich des DPOs an der Schwingung beteiligt ist, verifiziert werden. Dies lässt die Annahme zu, dass der in Kapitel 6.3.1 bereits bei Schwingungsverhalten des unbeschichteten Oszillators diskutierte Temperaturgradient für dieses ungewöhnliche Verhalten verantwortlich ist.

Vor Klärung einer möglichen Ursache für dieses Verhalten muss sichergestellt werden, dass nicht unterschiedliche Temperaturen während der Messung zu einer Verschiebung der Temperaturskala zwischen Heiz- und Kühlmessung zu einer Verfälschung der Ergebnisse führen. Insbesondere ist auszuschließen, dass der gemessene Effekt auf einen durch die Subtraktion der Leermessung bedingten systematischen Fehler zurückgeht.

Wie in Kapitel 4.1.3.3 diskutiert, kann bei hinreichend langer Wartezeit bis zur Einstellung des thermischen Gleichgewichts am DPO ein systematischer Fehler in der absoluten Temperatur als Ursache für die Unterschiede zwischen Heiz- und Kühlmessungen verhindert werden. Um den Einfluss der verwendeten Wartezeit zu untersuchen, wurde im 4. Messdurchgang die Reihenfolge der Messungen von zuvor CL1-ST-CL2-CL3-AS1-AS2 zu ST-CL1-AS2-CL3AS1-CL2 permutiert. Somit wurde die Wartezeit vor der jeweiligen Messung um bis zu 15 Minuten variiert, da die Einzelmessungen ebenfalls Zeit benötigen, während der sich die Temperaturverteilung weiter ändern könnte.

Es konnte keine signifikante Abhängigkeit gefunden werden, wie in Abbildung 6.20 exemplarisch für die CL2-Mode dargestellt ist. Die beobachtete Verschiebung von $\Delta f / f$ zwischen den beiden Durchgängen ist auf die zusätzliche thermische Auslagerung zurückzuführen und tritt auch zwischen Durchgang 2 und 3 auf (s. o.). Ein Effekt aufgrund der unterschiedlichen Wartezeit vor der jeweiligen Messung kann also nicht gefunden werden. Die beobachteten Unterschiede lassen sich somit nicht auf eine fehlerhafte Temperaturangabe zurückführen. 

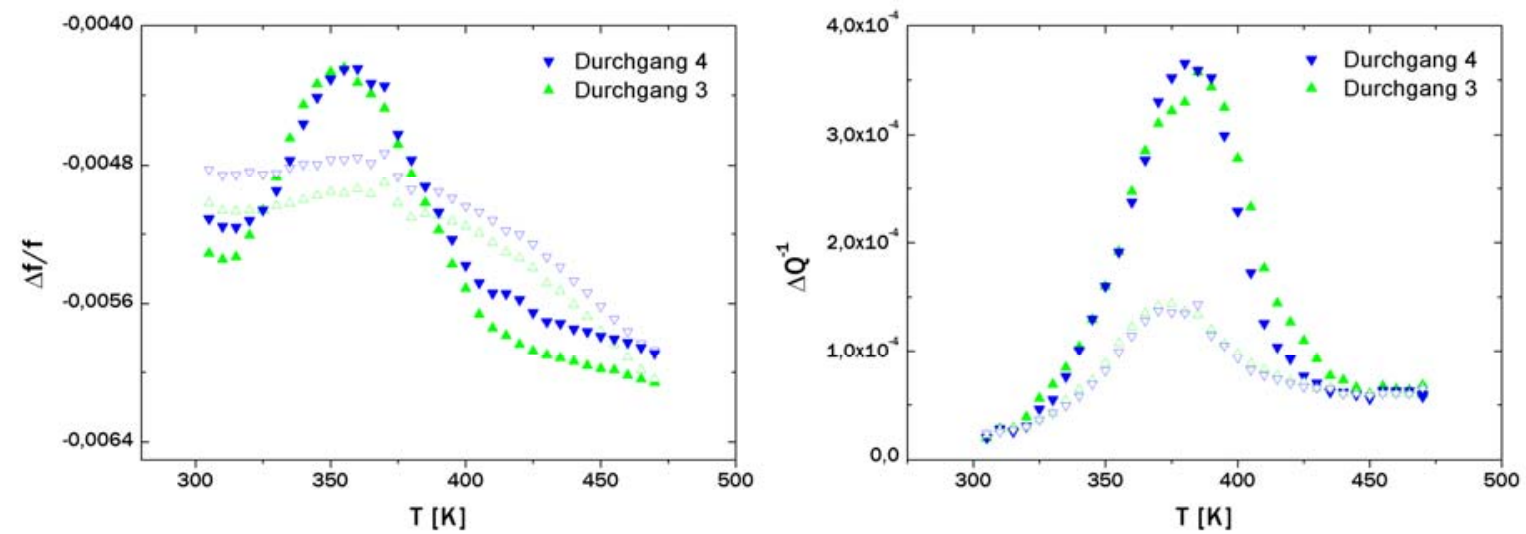

Abbildung 6.20: Relative Frequenzverschiebung und inverser Qualitätsfaktor der PEMA-Schicht gemessen an der CL2-Mode. Für die beiden Messungen wurden die verschiedenen Moden in unterschiedlicher Reihenfolge angefahren, s. d. Effekte durch verschieden lange Wartezeiten vor der Messung auszuschließen sind.

Zur weiteren Untersuchung dieser Abhängigkeit von Resonanzfrequenz und Dämpfung von der Heizrichtung wurden Experimente durchgeführt, bei denen die Heizrichtung im oberen Temperaturbereich um $410 \mathrm{~K}$ gewechselt wurde. In Abbildung 6.21 ist links der zeitliche Verlauf der Resonanzfrequenz der CL2-Mode bei schneller Änderung der Temperatur (Heiz- und Kühlrate $1 \mathrm{~K} / \mathrm{min}$ ) und anschließenden Haltephasen dargestellt. Die in rot eingetragene Resonanzfrequenz folgt grob der in schwarz dargestellten Temperatur am DPO-Halter. Nach dem Heizen liegt die Resonanzfrequenz für $T=410 \mathrm{~K}$ bei um etwa $0,05 \mathrm{~Hz}$ niedrigeren Werten als nach dem Kühlen (Die Frequenzskala ist fallend aufgetragen), was einer stärkeren Frequenzverschiebung gegenüber der Leermessung entspricht. Die in den obigen Ergebnissen der Messungen mit monotoner Temperaturänderung (z. B. in Abbildung 6.20) gefundene Tendenz wird also bestätigt. Der Effekt ist beim schnellen Temperaturwechsel jedoch schwächer ausgeprägt.

In der Dämpfung ist die Heizrichtungsabhängigkeit stärker ausgeprägt. Hier unterscheiden sich die Werte von $Q^{-1}(410 \mathrm{~K})$ um ca. $1 \cdot 10^{-5}$, je nachdem ob die Probe zuvor geheizt oder gekühlt wurde. Erneut bestätigen die Messungen die in den ursprünglichen Messungen beobachtete Tendenz: Die hier nach dem Heizen aufgenommenen Werte sind wie auch bei den Messungen mit monotoner Temperaturänderung größer als die nach einer Kühlphase bestimmten.

Der Vergleich mit den bei monotoner Temperaturführung aufgenommenen Werten zeigt sowohl für die Frequenz-, als auch für die Dämpfungsunterschiede, dass die Effekte hier beim schnellen Umschalten der Heizrichtung stark unterdrückt werden. Für Messdurchgang 4 findet man beim kontinuierlichen Heizen und anschließenden Kühlen für $T=410 \mathrm{~K}$ Unterschiede von $\Delta f=0,6 \mathrm{~Hz}$ und $\Delta Q^{-1}=5 \cdot 10^{-5}$. 

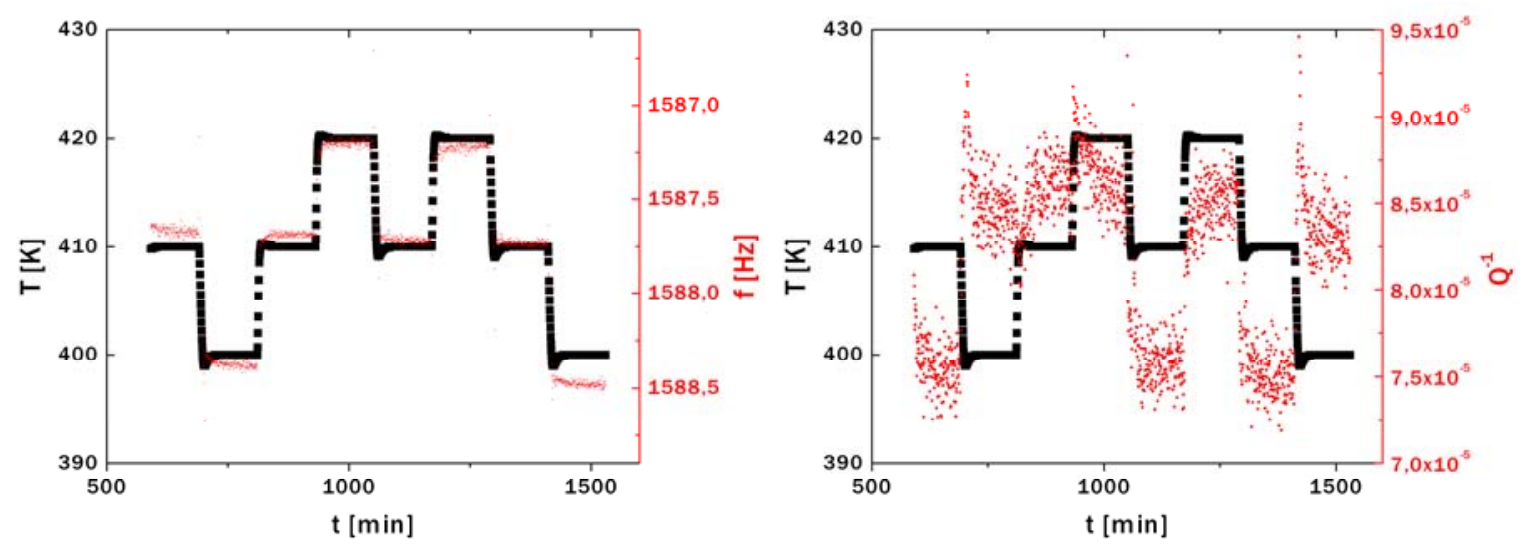

Abbildung 6.21: Zeitlicher Verlauf der an der CL2-Mode gemessenen Resonanzfrequenz (links) und Dämpfung (rechts) bei schnellem Heizen und Kühlen (Heizrate $1 \mathrm{~K} / \mathrm{min}$ ) und anschließenden Haltephasen. In schwarz ist die am Halter gemessene Temperatur aufgetragen. Während die Resonanzfrequenz wie erwartet dem Temperaturprofil folgt und nur leichte Abhängigkeit von der Vorgeschichte zeigt, ist der Wert von $\mathbf{Q}^{-1}$ von der direkten thermischen Vorgeschichte, bzw. der Heizrichtung abhängig.

Der Effekt ist also nicht nur von der Heizrichtung, sondern von der thermischen Vorgeschichte des Systems abhängig. Auch ist ein reiner Substrateffekt auszuschließen, da die Effekte in der Dämpfung des beschichteten Systems im Vergleich zum leeren Oszillators massiv verstärkt sind.

Als mögliche Ursache für die beobachteten Effekte bleibt auch hier der in Kapitel 6.3.1 diskutierte Temperaturgradient im schwingenden System. Die schnelle Änderung des Temperaturgradienten bei Umkehrung der Heizrichtung kann nun entweder dazu führen, dass im Film ein mikroskopischer Dämpfungsprozess einfriert bzw. nicht mehr vollständig zur Dämpfung beiträgt. Andererseits könnten die lokal unterschiedlichen Temperaturen des Paddels und auch des Films bewirken, dass bevorzugt Bereiche mit niedriger Dämpfung an der Schwingung beteiligt werden, s. d. die Gesamtdämpfung während der Kühlmessung verringert ist. Auch eine lokale Veränderung im Bereich der Einspannung des Oszillators, die zu verstärkter Energiedissipation an die Halterung führen kann, ist möglich. Da ein ähnliches Verhalten auch für Untersuchungen am Poly(butyl methacrylat) gefunden wurde, wird die Diskussion im Zusammenhang mit dort gewonnenen Ergebnissen in Kapitel 7.2 fortgesetzt.

In Abbildung 6.22 ist ein Teil der Ergebnisse für die unterschiedlichen Moden zusammengefasst. Aufgetragen sind die für alle Moden anhand von einfachen Gauß-Anpassungen mit zusätzlicher linearer Grundlinie bestimmten Maximalwerte der Dämpfungsdifferenz in Abhängigkeit von der mittleren Resonanzfrequenz. Die Farbkodierung entspricht dabei der bereits bekannten und kennzeichnet die verschiedenen Messdurchläufe. Volle Symbole zeigen die Daten der Heizmessungen, die leeren Symbole die der zugehörigen Kühlmessungen. Trotz der 
systematischen Ungenauigkeit der Bestimmung der Maximalwerte ${ }^{1}$ und der Streuung der ermittelten Resonanzfrequenzen unter $2 \mathrm{kHz}$ ist die oben bereits diskutierte Abweichung zwischen den Heiz- und den Kühlmessungen klar ersichtlich. Die Maxima der Kühlkurven liegen bei tieferen Temperaturen als die der Heizmessungen. Mit steigender Messfrequenz wird dieser Effekt geringer. Die Temperatur des Maximums der Kühlmessungen steigt mit steigender Messfrequenz stark an, während bei den Heizmessungen keine eindeutige Tendenz zu erkennen ist. Zum anderen führt die thermisch induzierte Veränderung der Proben, also die sukzessive Auslagerung dazu, dass sich die Temperaturen der maximalen Dämpfung für die Heizund die Kühlmessungen annähern. Mit zunehmender Auslagerung fällt die Maximumstemperatur der Heizmessung, während die der Kühlmessung zunimmt. Für die Schwingungsmoden mit Resonanzfrequenzen über $2 \mathrm{kHz}$ ist hier keine Abhängigkeit ersichtlich, hier ist die absolute Abweichung aber auch geringer.

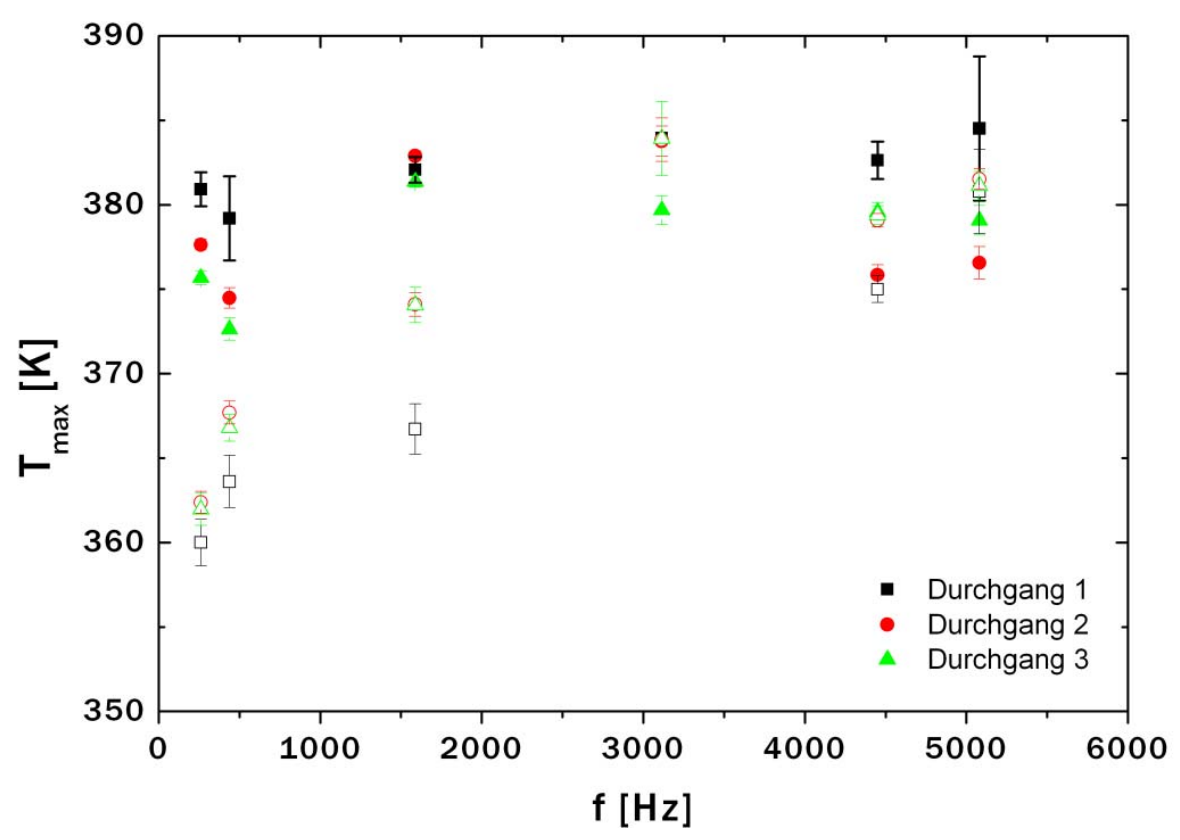

Abbildung 6.22: Mittels Gauß-Anpassungen bestimmte Temperaturen der maximalen Dämpfungsdifferenz für alle untersuchten Schwingungsmoden.

\footnotetext{
${ }^{1}$ Die Anpassung einer einfachen Gauß-Kurve kann den physikalisch richtigen Verlauf der Kurven nicht beschreiben (Vgl. Kapitel 2.1). Zur Bestimmung der Maximalwerte reicht sie hier aber aus.
} 


\subsection{Dielektrische Eigenschaften}

Aufgrund des Dipolmoments in den Ester-Seitengruppen ist bei den Poly(alkyl methacrylat)en neben der mechanischen Anregung auch die externe Ankopplung über elektrische Felder möglich. Die hier gewonnenen Ergebnisse werden im Folgenden dargestellt.

\subsubsection{Temperaturabhängigkeit}

Abbildung 6.23 zeigt einführend die Ergebnisse einer typischen Messung der dielektrischen Permittivität am Beispiel einer mit 400 Pulsen hergestellten PEMA-Schicht auf Silizium. Der links dargestellte Graph zeigt das dielektrische Verhalten beim zweiten Aufheizen der Probe, nachdem sie zuvor bereits einmal von 300 bis $400 \mathrm{~K}$ geheizt und dann wieder abgekühlt wurde. Die leeren Symbole zeigen den Real-, die vollen Symbole den Imaginärteil der Dielektrizitätszahl. Farblich kodiert ist die Temperatur während der Messung. Rechts ist das Verhalten beim darauf folgenden Abkühlen wiedergegeben. Zwischen den beiden Auftragungen sind keine signifikanten Unterschiede zu erkennen. Die Proben, die zuvor bei gleicher Temperatur ausgelagert wurden, zeigen also bei erneuter Temperaturbehandlung unterhalb dieser Auslagerungstemperatur keine weiteren irreversiblen Veränderungen.
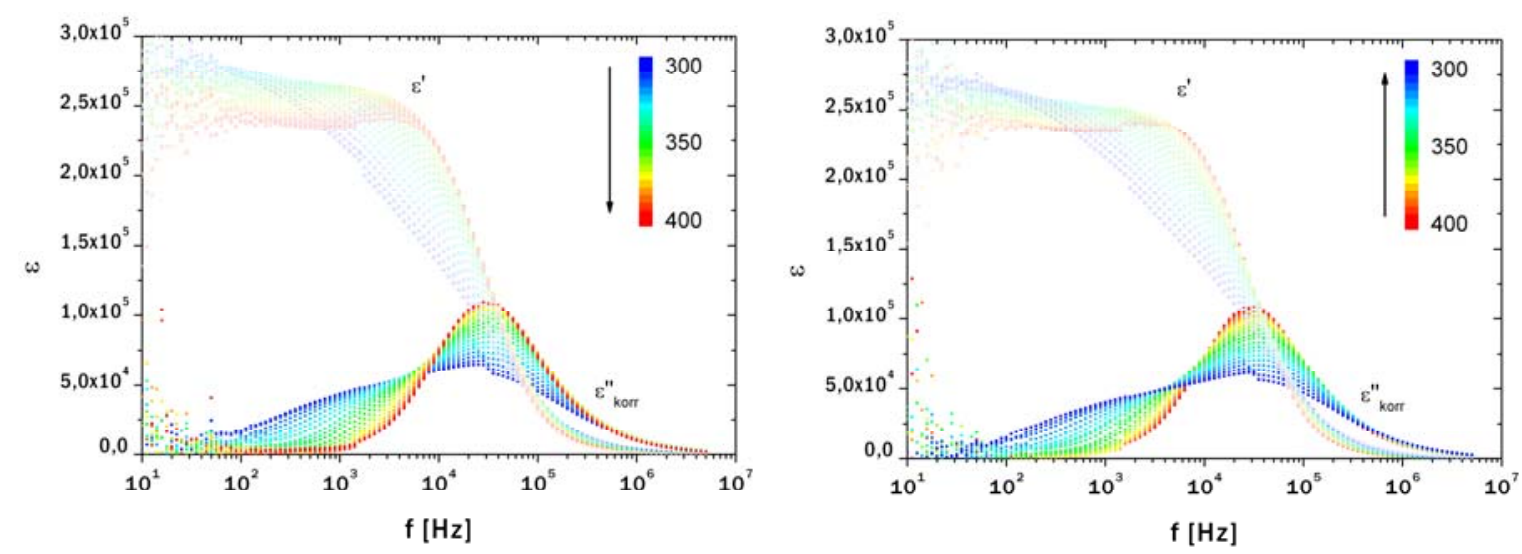

Abbildung 6.23: Real- (offene Symbole) und Imaginärteil (volle Symbole) der Permittivität eines mit 400 Laserpulsen deponierten PEMA-Films, der zuvor bei $400 \mathrm{~K}$ ausgelagert wurde, bei unterschiedlichen Temperaturen. Links ist die Heiz-, rechts die Kühlmessung gezeigt.

Bei fester, hoher Temperatur (rote Kurve) ist ein Debye-ähnliches Verhalten mit einer einfachen Relaxationszeit zu erkennen: Der Realteil der dielektrischen Funktion $\varepsilon^{6}$ bleibt bis zu Frequenzen von einigen $\mathrm{kHz}$ nahezu konstant und fällt dann mit steigender Frequenz ab. Der Imaginärteil $\varepsilon^{\text {“ }}$ zeigt dementsprechend ein Maximum bei $30 \mathrm{kHz}$. 
Bei niedrigen Temperaturen wird der Einfluss eines zweiten Relaxationsprozesses bei geringeren Frequenzen sichtbar. $\varepsilon^{6}$ knickt bereits bei im Vergleich zum Hochtemperaturmaximum etwa um eine Größenordnung verringerten Frequenzen ab und fällt dann weniger steil ab. Im dielektrischen Verlust $\varepsilon^{“}$ zeigt sich bei niedrigen Temperaturen ein erhöhter Beitrag der niederfrequenten Flanke, verbunden mit einem Absinken der Intensität im Maximum bei $30 \mathrm{kHz}$ und dessen spektraler Verbreiterung.

Diese Beschreibung diene hier zunächst nur zur grundlegenden Einführung in die Interpretation der dielektrischen Messungen. Details zu diesem reversiblen Verlauf werden in Kapitel 6.4.2.2 beschrieben. Da neben den mechanischen Eigenschaften auch die dielektrischen stark von der thermischen Vorbehandlung abhängen, ist es zunächst notwendig, den Einfluss der thermisch induzierten Veränderung zu beschreiben. Der Fokus liegt dabei auf den Untersuchungen des dielektrischen Verlustes.

\subsubsection{Abhängigkeit von der Temperatur-Vorgeschichte}

Um die bereits mit der mechanischen Spektroskopie beobachteten, thermisch induzierten Relaxationsprozesse zu untersuchen, wurden zwölf verschiedene Temperaturprofile mit einer Heizrate von $2 \mathrm{~K} / \mathrm{min}$ und unterschiedlichen Endtemperaturen durchfahren. Abbildung 6.24 zeigt das Ergebnis dieser Messreihe in einem Konturenplot. Auf der Abszisse ist die Messfrequenz aufgetragen, die Ordinate zeigt die Temperatur. Farblich kodiert ist $\varepsilon^{\text {" }}$ in logarithmischer Skala eingetragen. Unten links im Bild ist zunächst die erste Heizmessung zwischen 300 und $400 \mathrm{~K}$ nach der Herstellung der Proben bei Raumtemperatur dargestellt. Nach oben schließt sich die erste Kühlmessung (2) zurück auf $300 \mathrm{~K}$ an, gefolgt von der nächsten Heizmessung (3) usw. Die Darstellung der Ergebnisse der fünften Heizmessung (9) beginnt unten in der zweiten Spalte. Die jeweiligen Endtemperaturen sind notiert. Es zeigen sich drei klar trennbare Bereiche, die im Folgenden diskutiert werden. 


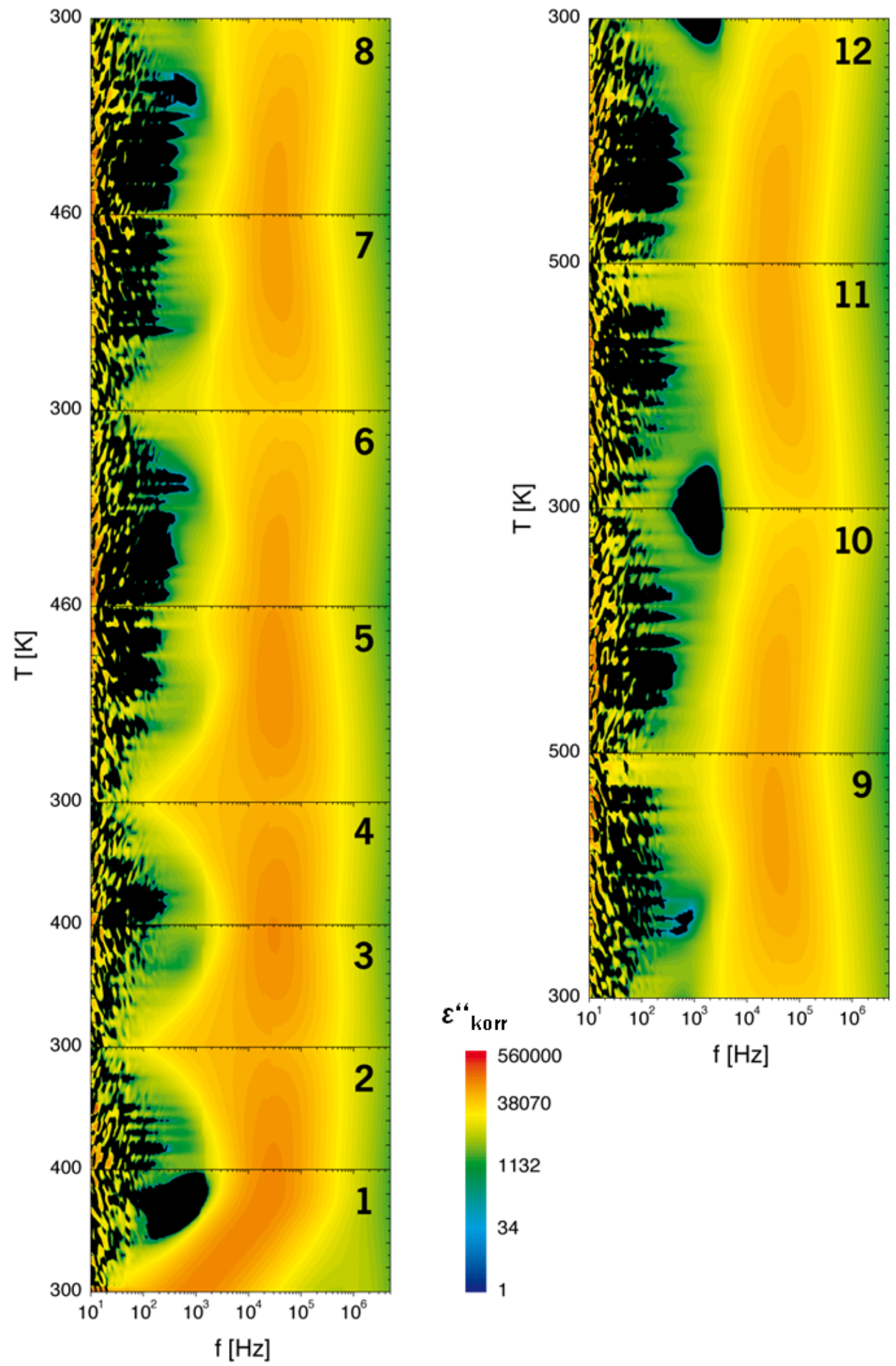

Abbildung 6.24: Dielektrischer Verlust in einer mit 400 Laserpulsen deponierten PEMA-Schicht zwischen $10 \mathrm{~Hz}$ und $5 \mathrm{MHz}$ bei thermischer Auslagerung zwischen 300 und $500 \mathrm{~K}$. Der Verlust ist in logarithmischer Farbskala aufgetragen. Der erste Messzyklus ist unten links, der letzte oben rechts aufgetragen. 


\subsubsection{Dielektrisches Verhalten bei erster Auslagerung}

Für die während des ersten Aufheizens (1) aufgenommenen Daten ist in Abbildung 6.24 ein starkes, symmetrisches Maximum zu erkennen, dass sich mit steigender Temperatur zu höheren Frequenzen verschiebt, seine Form, insbesondere Höhe und Breite aber nur wenig verändert. Die Messdaten dieses Durchgangs sind in Abbildung 6.25 detailliert aufgetragen. Jede Einzelkurve repräsentiert eine Messung bei fester Temperatur. Diese Messtemperatur ist gemäß der Farblegende abzulesen und läuft mit einer Schrittweite von $5 \mathrm{~K}$ zwischen $300 \mathrm{~K}$ und $400 \mathrm{~K}$. Zusätzlich eingetragen ist eine Anpassung der Kurven für unterschiedliche Temperaturen mit dem Imaginärteil einer Havriliak-Negami-Funktion (Formel 2.4). Der Asymmetrieparameter $\gamma$ der Havriliak-Negami-Funktion, der die Symmetrie der Kurve beschreibt, konnte konstant auf 1 gesetzt werden. Anpassungen, bei denen auch $\gamma$ als freier Parameter eingestellt werden konnte, führten nicht zu einer Verbesserung der Übereinstimmung der gemessenen Daten und der theoretischen Kurve, s. d. mit $\gamma=1$ die Cole-Cole-Funktion [125] ausreicht, um die Daten zu beschreiben.

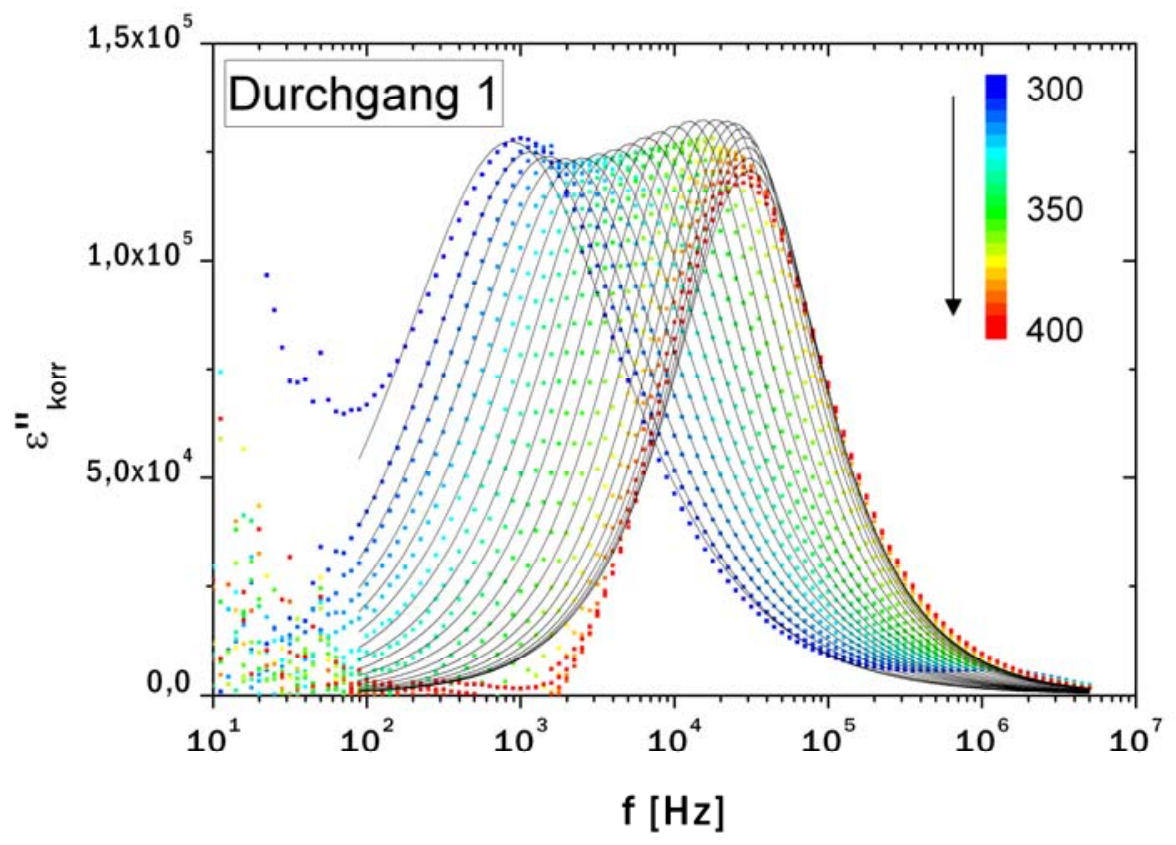

Abbildung 6.25: Dielektrischer Verlust $\varepsilon$ “ einer mit 400 Pulsen deponierten PEMA-Schicht während des ersten Heizdurchgangs. Zusätzlich eingetragen ist eine theoretische Anpassung nach Havriliak-Negami mit festem Asymmetrieparameter $\gamma=1$. Die zugehörigen Anpassungsparameter sind in Abbildung 6.26 aufgetragen. 

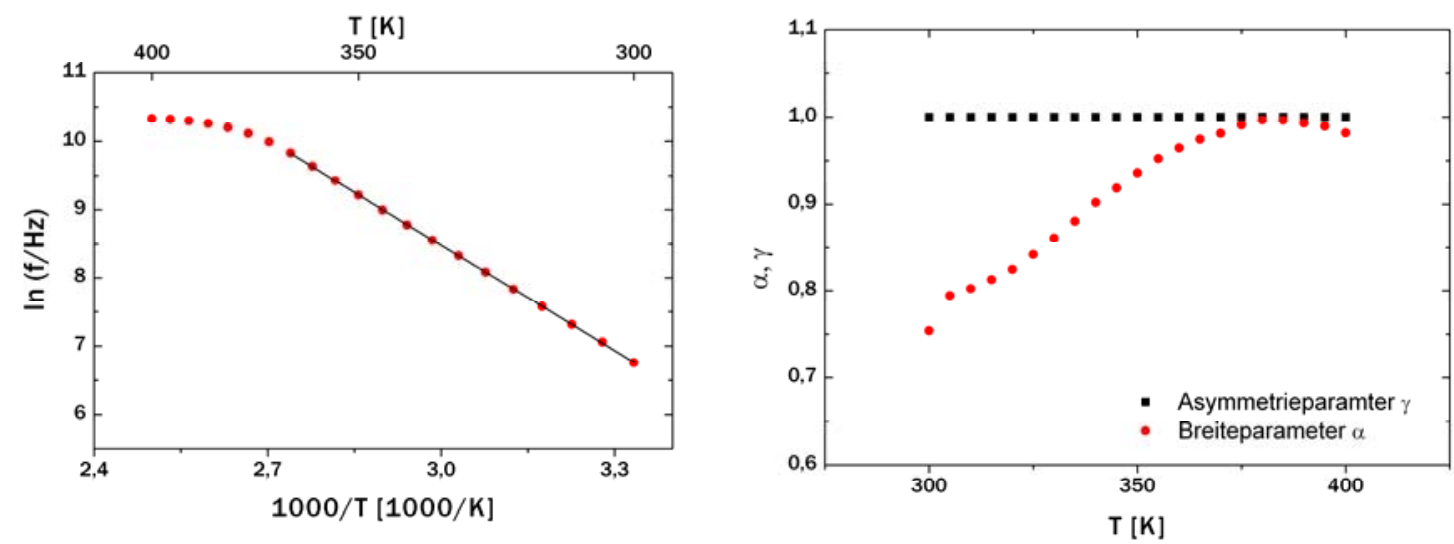

Abbildung 6.26: Anpassungsparameter der Havriliak-Negami-Funktion an die Kurven in Abbildung 6.25. Links ist die Frequenz des maximalen Verlustes in Arrhenius-Darstellung, rechts der Breiteparameter $\alpha$ und der Asymmetrieparameter $\gamma$ aufgetragen. Die Steigung im linken Bild lässt auf einen thermisch aktivierten Prozess mit $E_{\mathrm{a}}=0,45 \mathrm{eV}$ schließen.

Die zugehörigen Anpassungsparameter sind in Abbildung 6.26 eingetragen. Im linken Teil ist in einer Arrhenius-Darstellung die Maximumsfrequenz gegenüber der reziproken Temperatur aufgetragen. Ausgehend von Raumtemperatur ist zunächst ein linearer Verlauf zu erkennen, bevor die Maximumsfrequenz ab etwa $380 \mathrm{~K}$ mit steigender Temperatur nicht weiter zu höheren Werten verschoben wird. Die ebenfalls eingezeichnete Regressionsgerade liefert eine Aktivierungsenergie von $0,45 \mathrm{eV}$ für diese Frequenzverschiebung während des ersten Aufheizens. Im rechten Teil sind die Havriliak-Negami-Parameter eingetragen, wobei der Asymmetrieparameter $\gamma$, wie oben beschrieben, für alle Kurven auf 1 gesetzt wurde. Der in rot eingetragene Breiteparameter $\alpha$ nimmt beginnend von Raumtemperatur von $\alpha(300 \mathrm{~K})=0,75$ linear mit etwa $0,003 / \mathrm{K}$ auf $\alpha(370 \mathrm{~K})=1 \mathrm{zu}$. Oberhalb von $370 \mathrm{~K}$ fällt der Wert wieder leicht ab, was im Rahmen der Genauigkeit der Anpassung aber zu vernachlässigen ist. Die Form des Maximums nähert sich also mit steigender Temperatur der Form einer Debye-Relaxation an und behält diese auch für hohe Temperaturen.

\subsubsection{Reversibles Verhalten bei mittleren Temperaturen}

Wie in Abbildung 6.24 zu erkennen, ist der in Kapitel 6.4.2.1 diskutierte Verlauf nicht reversibel. Bereits beim ersten Abkühlen (2) bleibt auch bei Raumtemperatur ein signifikanter Anteil im dielektrischen Verlust bei Frequenzen über $10 \mathrm{kHz}$ zurück. So sind bei niedrigen Temperaturen zwei Maxima zu erkennen. Die Intensität des Maximums bei niedrigen Frequenzen um $1 \mathrm{kHz}$ ist dabei im Vergleich zur Neukurve stark reduziert. Dieses Verhalten ist bis zum einmaligen Auslagern bei 460 K, also bis zum Ende von Durchlauf 5 reversibel, s. d. im Fol- 
genden stellvertretend die in dieser Heizmessung aufgenommenen Ergebnisse dargestellt werden.

Abbildung 6.27 zeigt den dielektrischen Verlust während des fünften Messdurchgangs, also des dritten Heizdurchgangs. Bei niedrigen Temperaturen (blaue Kurve) sind klar zwei Beiträge zu erkennen. Die Schulter bei etwa $1 \mathrm{kHz}$ wird im Folgenden als niederfrequenter Beitrag (low frequency, LF) bezeichnet. Sie repräsentiert den aus der Neukurve bekannten Prozess, der anhand der auftretenden Frequenzen als $\beta$-Relaxation identifiziert werden $[126,127]$ kann. Bei etwa $30 \mathrm{kHz}$ ist ein Hochfrequenzmaximum (high frequency, HF) zu erkennen. Mit steigender Temperatur wird der NF-Beitrag zu höheren Frequenzen, also zu Werten des HFMaximums verschoben, s. d. er nur bis zu einer Temperatur von etwa $350 \mathrm{~K}$ klar von diesem zu trennen ist. Die Frequenz des HF-Maximums ist von der Temperatur nahezu unabhängig, nur die Stärke nimmt mit steigender Temperatur bis etwa $380 \mathrm{~K}$ zu. Für höhere Temperaturen wird der maximale dielektrische Verlust wieder schwächer.

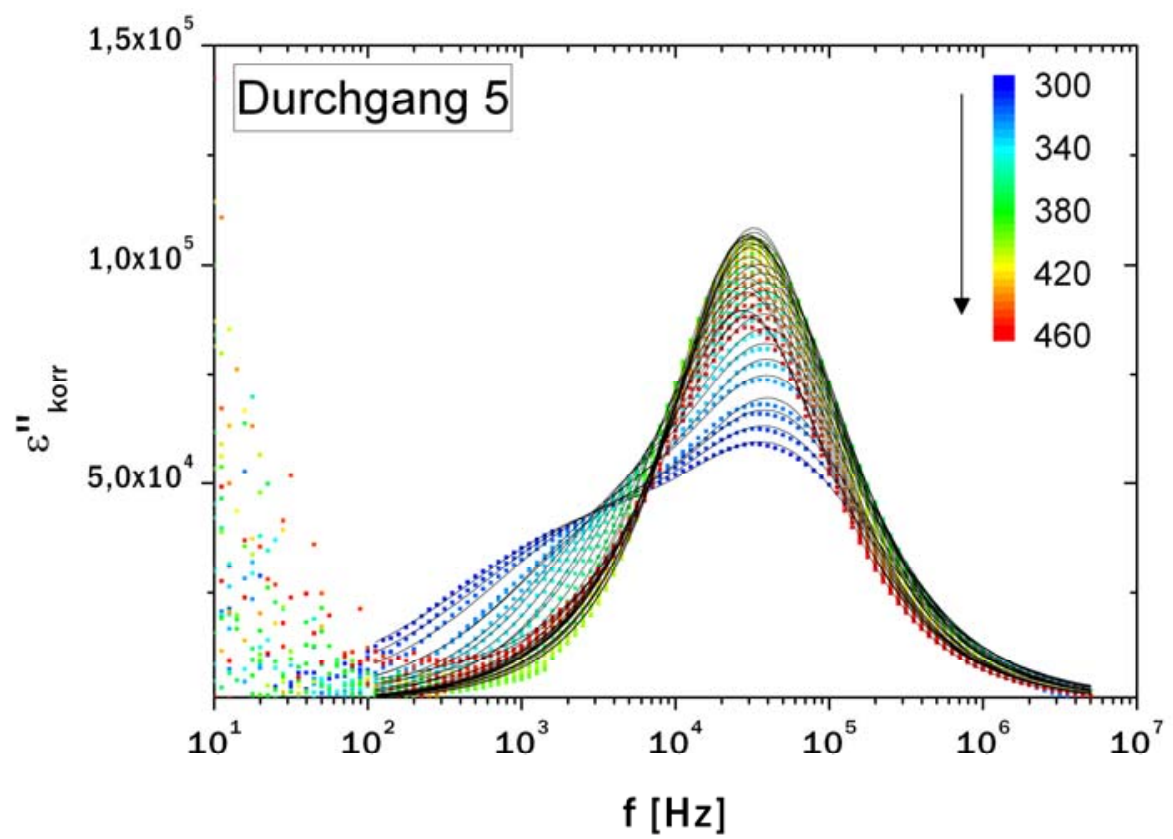
Abbildung 6.27: Nach der Auslagerung bei mittleren Temperaturen ist im dielektrischen Verlust einer mit
$400 \mathrm{Laserpulsen}$ hergestellten PEMA-Schicht bei niedrigen Temperaturen (blaue Kurve) zunächst ein Maximum bei
$30 \mathrm{kHz}$ (HF) mit stark ausgeprägter Schulter bei etwa $1 \mathrm{kHz}$ (NF) zu erkennen. Mit steigender Temperatur ver-
schmelzen Schulter und HF-Maximum zu nur noch einem Maximum. Zusätzlich eingetragen sind numerische Anpas-
sungen mit einer Summe zweier Cole-Cole-Funktionen. Die Anpassungsparameter sind Abbildung $6.28 \mathrm{f}$. zu entneh-
men.

Zusätzlich eingezeichnet ist wieder eine theoretische Anpassung nach Havriliak-Negami. Hier wurde die Summe zweier Cole-Cole-Funktionen verwendet, $\gamma=1$ also wieder als konstant festgelegt. Besonders im Übergangsbereich um $350 \mathrm{~K}$ ist die Trennung beider Maxima und 
insbesondere die Angabe einer festen Temperatur, ab der die Maxima verschmolzen sind, schwierig, s. d. die ermittelten Parameter sicherlich nicht eindeutig sind.

In Abbildung 6.28 ist als erster Anpassungsparameter die dielektrische Relaxationsstärke, also die direkte Gewichtung der jeweiligen Havriliak-Negami-Funktionen in der Summenfunktion eingetragen. Die roten Punkte repräsentieren die Stärke des niederfrequenten Anteils (NF), die mit steigender Temperatur linear abnimmt. Ab $360 \mathrm{~K}$ liefert der Anpassungsalgorithmus dann nur noch einen Anteil für den in grün dargestellten HF-Prozess, der ab Raumtemperatur linear ansteigt, bei $360 \mathrm{~K}$ maximal ist und für höhere Temperaturen ebenfalls zurückgeht. 


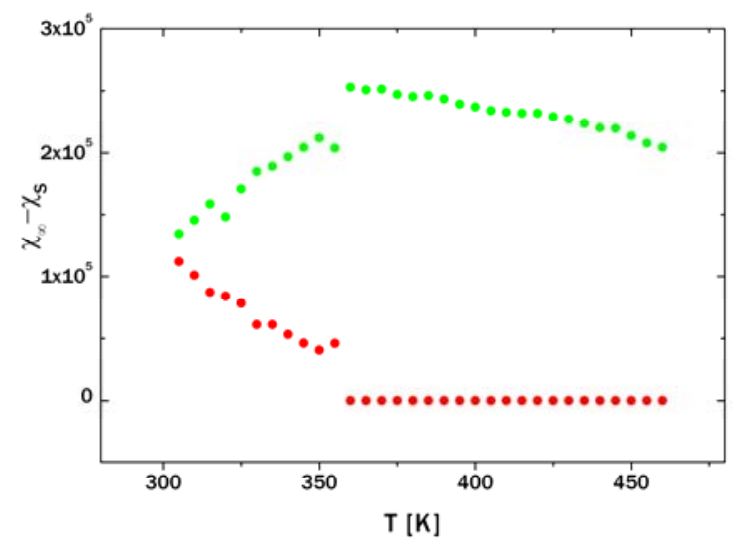

Abbildung 6.28: Dielektrische Relaxationsstärke bestimmt aus der Anpassung einer Summe zweier Cole-ColeFunktionen an die Messungen des dielektrischen Verlustes in Abbildung 6.27. Die grünen Punkte zeigen den Vorfaktor der Cole-Cole-Funktion mit Maximum bei hohen Frequenzen, die roten Punkte repräsentieren den Anteil der Funktion mit Maximum bei niedrigen Frequenzen. Ab $360 \mathrm{~K}$ reicht eine einfache Cole-Cole-Funktion aus, um die Daten zu beschreiben.
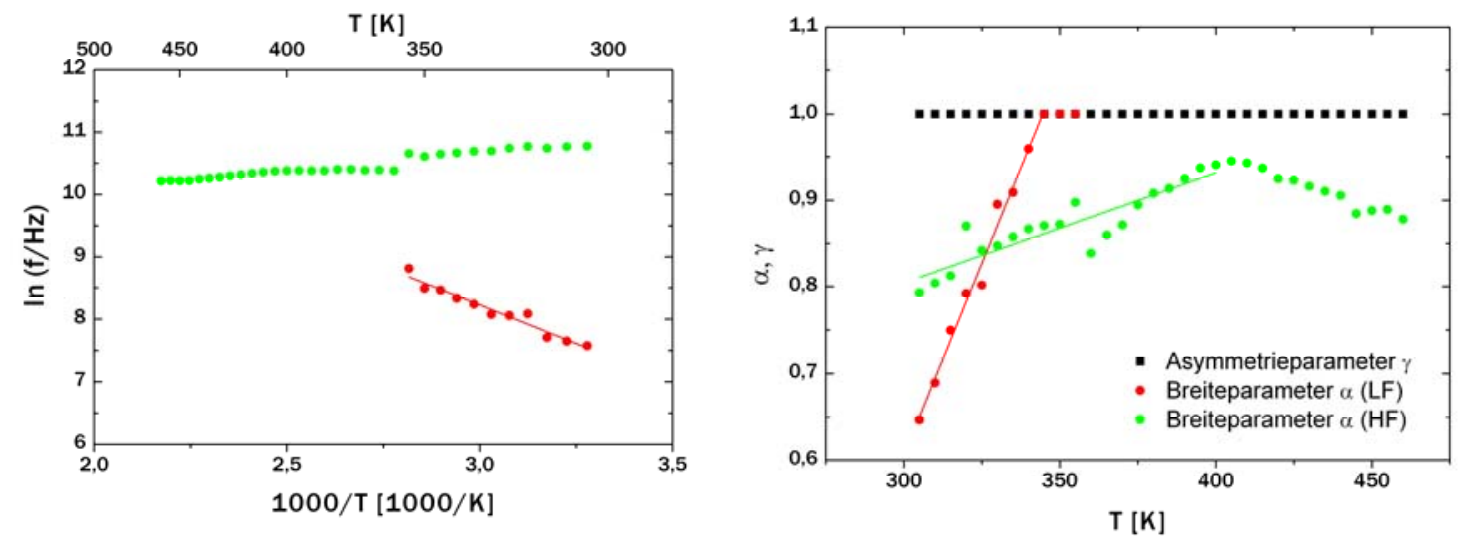

Abbildung 6.29: Anpassungsparameter der in Abbildung 6.27 eingetragenen Havriliak-Negami-Funktionen. In grün sind die Werte für das Maximum bei niedrigen Frequenzen (LF), in rot die für das Maximum bei hohen Frequenzen (HF) eingetragen. Links sind die Maximumsfrequenzen in Arrheniusdarstellung aufgetragen, rechts Asymmetrie- und Breiteparameter.

Die weiteren Anpassungsparameter sind in Abbildung 6.29 dargestellt. Im linken Teil ist die Maximumsfrequenz für die verschiedenen Messtemperaturen in einer Arrheniusdarstellung aufgetragen. Die grünen Punkte repräsentieren dabei die Frequenzen des hochfrequenten Maximums. Hier fallen die Werte mit zunehmender Temperatur leicht ab, was einem AntiArrhenius-Verhalten entspricht. Die Frequenzen des niederfrequenten Beitrags (LF) sind in rot eingetragen. Hier ist ein lineares Arrheniusverhalten mit einer Aktivierungsenergie von $0,21 \mathrm{eV}$, die über die ebenfalls eingezeichneten Regressionsgerade bestimmt wurde, zu erkennen. Diese im Vergleich zu Werten, die an Volumenmaterial gewonnen wurden $(0,8$ bis $1,3 \mathrm{eV}$ [127, 128]) geringe Aktivierungsenergie lässt sich durch unterschiedliche Modifikationen erklären. Zum einen besteht die Möglichkeit, dass durch den Prozess der Laserdeposition lo- 
kal das freie Volumen im Material erhöht ist, was die mit der Schwingung der Seitengruppen verbundene $\beta$-Relaxation vereinfacht. So führt das Abspalten vereinzelter Seitengruppen zu einer Absenkung der Aktivierungsenergie der verbleibenden Dipole.

Die Formparameter der Havriliak-Negami-Funktion sind im rechten Teil der Abbildung eingetragen. Wieder sind die Punkte des Beitrags bei niedrigen Frequenzen rot, die des hochfrequenten Maximums entsprechend grün eingetragen. Für beide Maxima ist mit steigender Temperatur bis etwa $400 \mathrm{~K}$ eine Annäherung an ein Debye-typisches Verhalten zu erkennen. Zwischen $300 \mathrm{~K}$ und $350 \mathrm{~K}$ steigt der Breiteparameter des LF-Maximums mit etwa 0,009/K schnell von 0,65 auf 1. Der Parameter $\alpha$ des hochfrequenten Maximums steigt langsamer mit $0,001 / \mathrm{K}$ von 0,8 bei Raumtemperatur auf 0,95 bei $400 \mathrm{~K}$ und fällt danach wieder ab.

Wie oben bereits bemerkt, kann diese Anpassung sicherlich keine eindeutige Beschreibung des dielektrischen Verlustes liefern. Die Schwäche der Anpassung zeigt sich besonders anhand der Sprünge im Verschmelzungsbereich der beiden Maxima bei etwa $350 \mathrm{~K}$. Dennoch beschreiben die Anpassungen sehr gut den Verlauf der einzelnen Kurven und die bestimmte Temperaturabhängigkeit der Parameter kann als charakteristisch angesehen werden.

\subsubsection{Irreversible Veränderungen bei hohen Temperaturen}

Die Auslagerung bei hohen Temperaturen führt zu weiteren Veränderungen der Probe, die eine Veränderung des dielektrischen Verlustes bewirken. Abbildung 6.30 zeigt die Ergebnisse des 11. Messdurchgangs, also nach Auslagerung bei $500 \mathrm{~K}$. Hier ist für alle Temperaturen nur ein Maximum zu erkennen, das jetzt aber mit steigender Temperatur zu geringeren Frequenzen verschoben wird. Zusätzlich eingetragen sind die Ergebnisse der Anpassung einer einfachen Cole-Cole-Funktion.

Anhand der Anpassungsparameter der Cole-Cole-Funktion kann der Verlauf der Kurve charakterisiert werden: Die in Abbildung 6.31 dargestellte Relaxationsstärke ist über den gesamten Temperaturbereich geringer als die des HF-Prozesses in Messdurchgang 5. Der dielektrische Verlust im Material ist also bedeutend schwächer, was vermutlich auf Degradation des Polymers zurückzuführen ist. Mit steigender Temperatur nimmt der maximale dielektrische Verlust zwar auch noch bei im Vergleich zum fünften Messdurchgang um etwa $100 \mathrm{~K}$ erhöhten Temperaturen zu, bleibt aber weit unter den hier gemessenen Werten. Erst ab $460 \mathrm{~K} \mathrm{nimmt}$ die Relaxationsstärke mit weiterer Temperaturerhöhung ab. Diese unterschiedlichen absoluten 
Temperaturen, bei denen sich die dielektrische Relaxationsstärke ändert, lassen darauf schließen, dass die zum dielektrischen Verlust führenden Prozesse hier grundlegend verschieden sind.

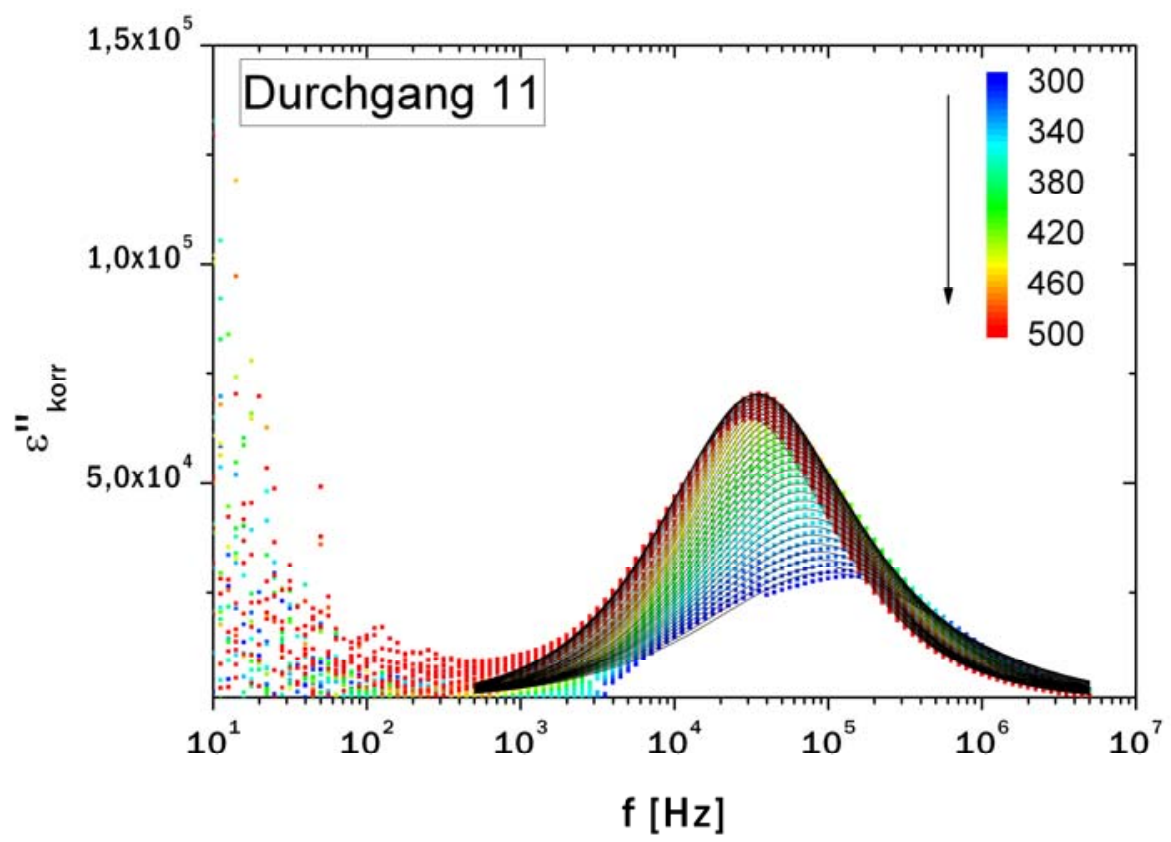

Abbildung 6.30: Nach der Auslagerung bei hohen Temperaturen ist der niederfrequente Beitrag im dielektrischen Verlust einer mit 400 Laserpulsen hergestellten PEMA-Schicht für alle Temperaturen unterdrückt. Zusätzlich eingetragen ist die numerische Anpassung mit einer Cole-Cole-Funktion. Die Anpassungsparameter sind in Abbildung 6.32 dargestellt.

Ungewöhnlich ist der Verlauf der Frequenz maximalen dielektrischen Verlustes, die in Abbildung 6.32 links in einer Arrhenius-Auftragung dargestellt ist. Bereits bei Raumtemperatur zeigt der dielektrische Verlust der PEMA-Schicht eine Resonanz bei Wechselströmen sehr hoher Frequenz von $200 \mathrm{kHz}$. Mit steigender Temperatur verschiebt sich das Maximum dann $\mathrm{zu}$ tieferen Frequenzen. Das für das HF-Maximum in Kapitel 6.4.2.2 angedeutete AntiArrhenius-Verhalten ist hier deutlich ausgeprägt. Ein für unbehandeltes PEMA erwarteter thermisch aktivierter Prozess kann nicht mehr gefunden werden. Die Ursachen für dieses ungewöhnliche Verhalten der Probe werden mit den Ergebnissen bzgl. der Gleichstromleitfähigkeit aus dem folgenden Kapitel erst im zusammenfassenden Kapitel 6.6 diskutiert. Aber bereits hier wird klar, dass die UV-Bestrahlung während der Laserdeposition und die anschließende thermische Behandlung, die dielektrischen Eigenschaften des Polymers im Vergleich zum Volumenmaterial massiv verändern. 


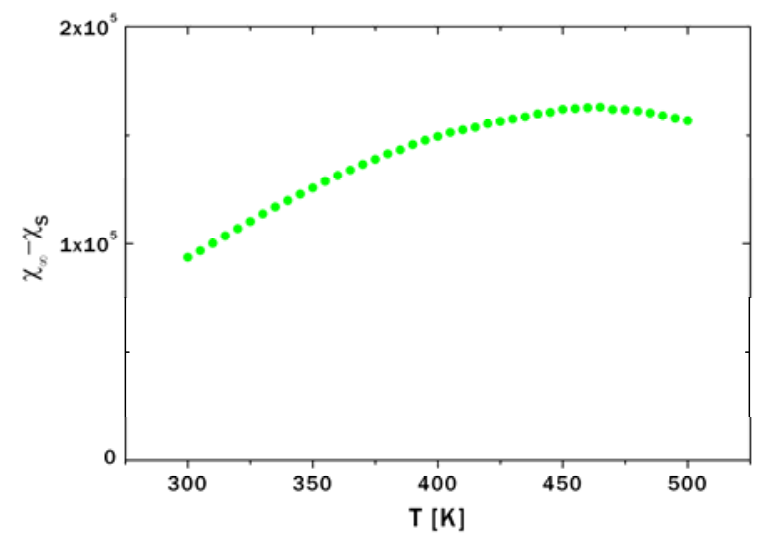

Abbildung 6.31: Die anhand der Messdaten aus Durchgang 11 bestimmte dielektrische Relaxationsstärke ist durch die Auslagerung bei hohen Temperaturen im Vergleich zu Durchgang 5 stark reduziert. Innerhalb des Messdurchgangs kann ein Ansteigen der Intensität bis $450 \mathrm{~K}$ beobachtet werden, oberhalb von $450 \mathrm{~K}$ fällt sie dann wieder ab.
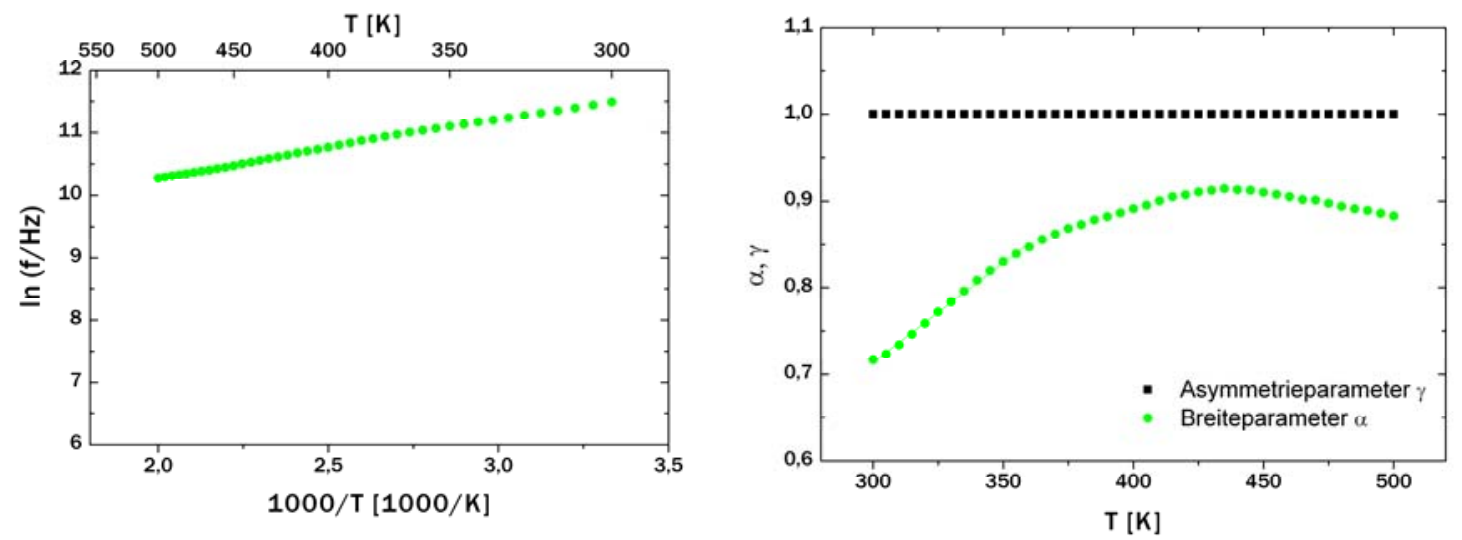

Abbildung 6.32: Anpassungsparameter der Cole-Cole-Funktion an die Daten aus dem 11. Messdurchgang. Links ist die Frequenz des maximalen dielektrischen Verlustes in einer Arrhenius-Darstellung aufgetragen, die hier ein starkes Anti-Arrhenius-Verhalten zeigt. Im linken Teil sind Asymmetrie- und Breiteparameter dargestellt.

Die in Abbildung 6.32 rechts dargestellten Werte für die Asymmetrie und Breite des Maximums entsprechen den bekannten Verläufen. Gemäß der Cole-Cole-Funktion ist für alle Temperaturen $\gamma=1$. Der Verlauf des Breiteparameters lässt sich in drei Bereiche aufteilen: Von Raumtemperatur bis $350 \mathrm{~K}$ steigt er zunächst mit $0,002 / \mathrm{K}$ von 0,75 bis auf 0,85 an, steigt dann langsamer bis auf 0,9 bei $435 \mathrm{~K}$ und fällt anschließend wieder ab.

\subsubsection{Gleichspannungsleitfähigkeit}

In Abbildung 6.33 ist der temperaturabhängige Gleichspannungsanteil des Leitwerts in einem Arrhenius-Diagramm dargestellt. Die Werte wurden aus dem jeweiligen Mittelwert der gemessenen Leitwerte für niedrige Frequenzen zwischen 10 und $20 \mathrm{~Hz}$ bestimmt. Aufgetragen sind die Kurven für alle zwölf Messdurchgänge, im linken Graphen zeigen die vollen Symbo- 
le wieder die Ergebnisse der Heizmessungen. Die leeren Symbole im rechten Diagramm repräsentieren die Messwerte der Kühlmessungen.
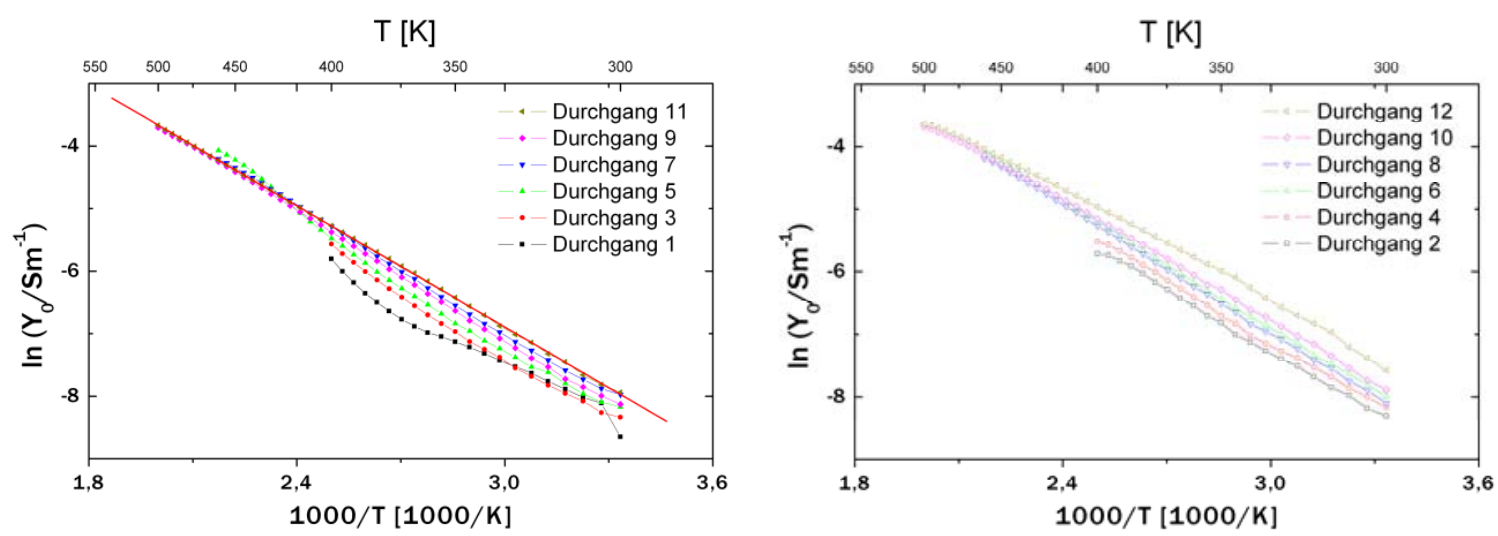

Abbildung 6.33: Arrheniusdarstellung des Gleichspannungsanteils des Leitwertes einer mit 400 Pulsen hergestellten PEMA-Probe für unterschiedliche Temperaturvorbehandlungen. Die Werte wurden bestimmt aus dem Mittelwert der Leitwerte für niedrige Frequenzen zwischen 10 und 20 Hz. Im linken Graph sind die während der Heizmessungen, im rechten Graph die während der Kühlmessungen aufgenommenen Werte dargestellt. Aus der Steigung der an die Werte des letzten Heizdurchgangs angepassten Regressionsgeraden ergibt sich eine Aktivierungsenergie von 0,28 eV.

Für die letzte Heizmessung (Durchgang 11) in Abbildung 6.33 ist zusätzlich eine Regressionsgerade eingetragen, die den Verlauf der Messpunkte sehr genau beschreibt. Hier ist von einem einfach thermisch aktivierten Leitungsmechanismus auszugehen. Die Aktivierungsenergie kann zu $E_{\mathrm{A}}=0,28 \mathrm{eV}$ bestimmt werden. Die Leitwerte der früheren Messdurchläufe zeigen Abweichungen vom linearen Verhalten in der Arrhenius-Auftragung, die auf thermisch induzierte Relaxationsprozesse im Material zurückzuführen sind. Mit steigender voriger Auslagerungstemperatur nehmen diese Abweichungen ab und die Kurven nähern sich dem linearen Verlauf. Besonders stark sind die Abweichungen vom linearen Verhalten an der wie hergestellten Probe zu erkennen (schwarze Quadrate). Bis zu einer Temperatur von etwa $355 \mathrm{~K}$ tritt lineares Verhalten mit verringerter Steigung auf (Aktivierungsenergie: 0,20 eV). Oberhalb dieser Temperatur zeigt sie einen gekrümmten Verlauf, was auf das Einsetzen weiterer Relaxationsprozesse schließen lässt.

Der Verlauf der in den Kühlmessungen bestimmten Leitwerte entspricht im Wesentlichen dem der aus den Heizmessungen bekannten. Der Effekt der thermisch induzierten Relaxation kann hier jedoch natürlich nicht beobachtet werden. Stattdessen zeigen sich weitgehend lineare Kurven, die mit steigender Auslagerungstemperatur zu höheren Absolutwerten verschoben sind. Besonders stark ist diese Verschiebung bei der letzten aufgenommenen Messung, nachdem die Probe das zweite Mal auf $500 \mathrm{~K}$ ausgelagert wurde. Hier ist von starken Veränderungen im Probenmaterial und partiellem Masseverlust auszugehen. 
Die Gleichstromleitfähigkeit liegt für sämtliche Messdurchgänge zwischen $Y_{0}(300 \mathrm{~K})=1 \cdot 10^{-4} \mathrm{Sm}^{-1}$ und $Y_{0}(500 \mathrm{~K})=3 \cdot 10^{-2} \mathrm{Sm}^{-1}$ und ist damit sehr hoch im Vergleich $\mathrm{zu}$ dem Literaturwert von PEMA-Volumenmaterial. Dieser beträgt im Temperaturbereich zwischen 340 und $500 \mathrm{~K}$ zwischen $2 \cdot 10^{-12} \mathrm{Sm}^{-1}$ und $2 \cdot 10^{-10} \mathrm{Sm}^{-1}$ [126], ist also um mindestens 8 Größenordnungen geringer als der des laserdeponierten Materials. Eine mögliche Ursache der hohen Leitfähigkeit können bewegliche, geladene Fragmente im Material sein, die auf den Ablationsprozess zurückzuführen sind. Erste Ergebnisse mittels Massenfeinbestimmung [129] lassen auf einen hohen Anteil von mehrfach ionisierten Fragmenten schließen, die als mögliche Ladungsträger in Frage kommen. Das hier beobachtete Arrheniusverhalten ließe sich dann durch die Kettendiffusion limitierte Ionenleitung erklären.

\subsection{Strukturelle Änderungen bei Auslagerung}

Um die mikroskopischen Ursachen der thermisch induzierten Zunahme der Dämpfung zu untersuchen, wurden während des Heizens einer laserdeponierten Probe Röntgenexperimente durchgeführt. Wäre das Ausheilen freien Volumens, welches durch den Prozess der Laserdeposition in den Schichten vorhanden sein könnte, der primäre Prozess [16], so sollten in den Röntgendiffraktogrammen bei unterschiedlichen Temperaturen signifikante Unterschiede auftreten.

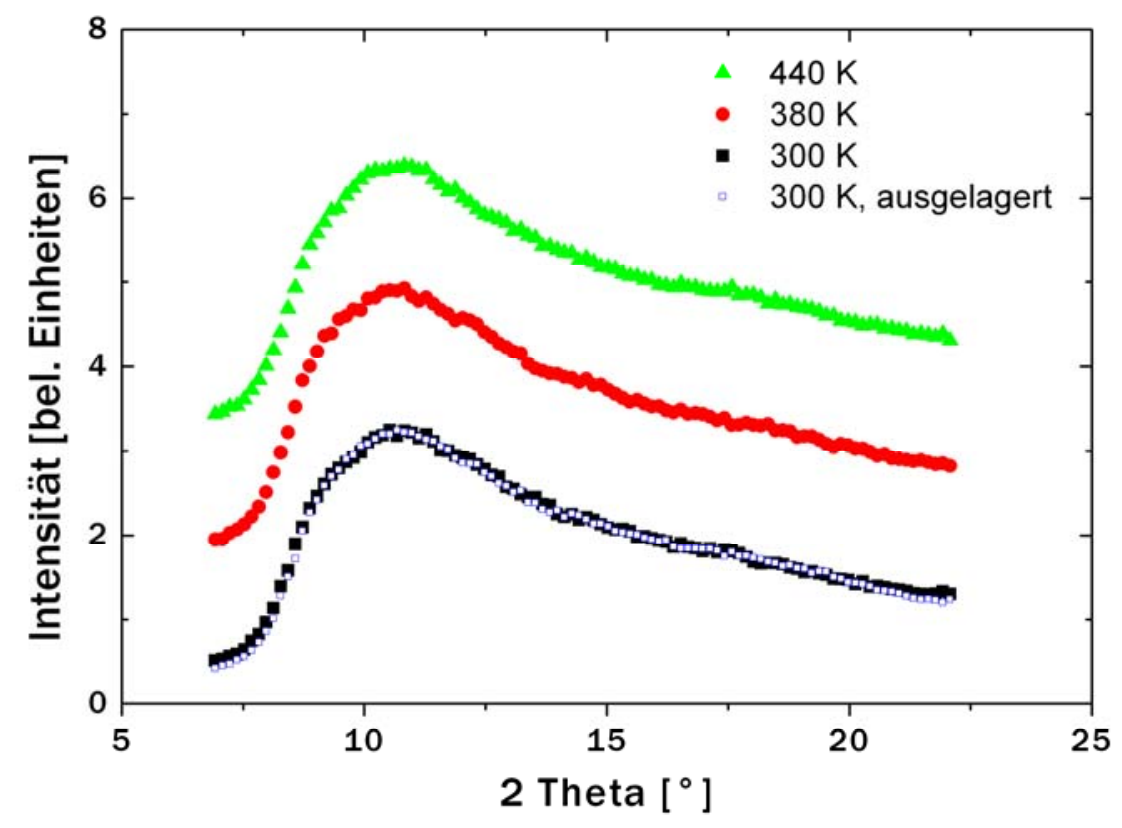


Abbildung 6.34: Bei unterschiedlichen Temperaturen aufgenommene Röntgendiffraktogramme einer mit 800 Pulsen hergestellten PEMA-Schicht. Sowohl beim Vergleich der Kurven bei unterschiedlichen Temperaturen, als auch beim Vergleich der Messung an der unbehandelten und an der ausgelagerten Probe bei Raumtemperatur sind keine signifikanten Unterschiede zu erkennen. Zur Übersichtlichkeit sind die bei unterschiedlichen Temperaturen aufgenommenen Kurven verschoben.,

In Abbildung 6.35 ist die Morphologie einer bei $500 \mathrm{~K}$ ausgelagerten PEMA-Schicht, die mit 800 Pulsen deponiert wurde, dargestellt. Im Vergleich mit den Aufnahmen der Morphologie einer frisch hergestellten Probe (Abbildung 5.1 auf Seite 60) fallen keine signifikanten Unterschiede auf. Eine Glättung der Filme, die bei Deposition von PMMA auf über die Glasübergangstemperatur geheizte Substrate gefunden wurde [15], kann hier nicht beobachtet werden. Das dazu notwendige Fließen des Inhalts ganzer Droplets wird durch die Haut bestehend aus der vernetzten Schichtkomponente unterdrückt.
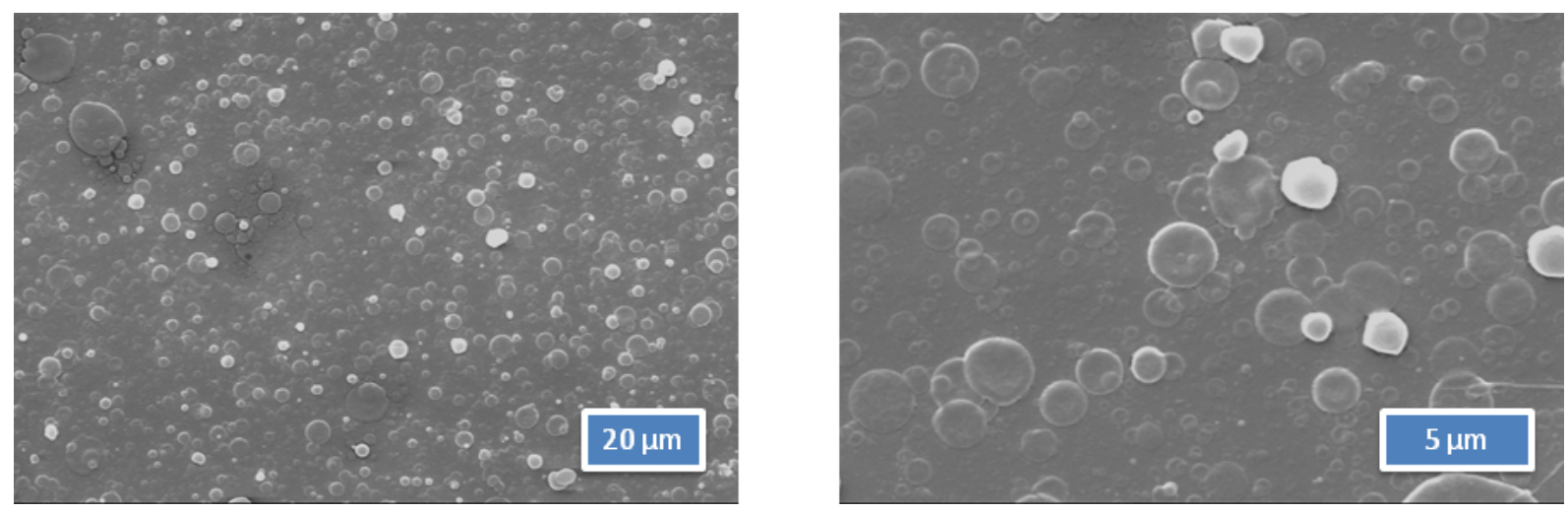

Abbildung 6.35: Rasterelektronenmikroskopische Aufnahmen der Oberflächenmorphologie einer mit 800 Pulsen laserdeponierten PEMA-Schicht, die bei $500 \mathrm{~K}$ ausgelagert wurde. Der Vergleich mit der Morphologie der Proben, die nicht ausgelagert wurden (Abbildung 5.1 auf Seite 60) zeigt keine signifikante Veränderung der Morphologie durch die Auslagerung.

\subsection{Zusammenfassende Diskussion der Relaxationseigenschaften}

Die zuvor gewonnenen Ergebnisse zeigen starke Änderungen der Filmeigenschaften bei Temperaturbehandlung. Dabei ist zu unterscheiden zwischen einer thermisch induzierten Relaxation, also der irreversiblen Veränderung der Probe durch Auslagerung bei hohen Temperaturen und der Temperaturabhängigkeit einer bereits relaxierten Probe, die keiner weiteren irreversiblen Veränderung unterzogen wird. 


\subsubsection{Thermisch induzierte Relaxation}

Sowohl die mechanischen, als auch die dielektrischen Eigenschaften des laserdeponierten Poly(ethyl methacrylats) zeigen eine starke Abhängigkeit von der thermischen Behandlung, die sich in irreversibler Veränderung der Probeneigenschaften darstellt.

Die mechanischen Messungen, sowohl mit PPXR, als auch mit den Torsionsmoden des DPOs bestimmt, liefern hier konsistente Ergebnisse. Speicher- und Verlustmodul steigen mit zunehmender Auslagerungstemperatur stark an, während Form und Lage der beobachteten Maxima weitestgehend temperaturunabhängig sind. Erst bei großen Auslagerungstemperaturen treten starke Veränderungen auch im Temperaturbereich weit über $T_{\mathrm{g}}$ auf. Die geringe Abhängigkeit der Form der Maxima lässt darauf schließen, dass durch die Auslagerung zunächst keine Änderung am grundsätzlichen zugrundeliegenden Relaxationsmechanismus induziert wird. Dennoch werden zum einen eine Härtung und zum anderen eine Zunahme der Energiedissipation im Material erreicht.

Die auftretenden Veränderungen sind dabei nicht mit einem Alterungsprozess, wie in Kapitel 2.1 beschrieben, zu verwechseln, da hier stets der Bereich der Schmelze oberhalb der Glasübergangstemperatur erreicht wurde. Aging-Prozesse werden bereits unterhalb der Glastemperatur aktiviert [130] und führen im Modell der Potentiellen-Energie-Landschaft weiterhin zum Absinken der Konfigurationsenergie und somit zur Zunahme des Speicher-, aber zur Abnahme des Verlustmoduls [62]. Solche Prozesse könnten hier nur bei den Neukurven beobachtet werden, sie werden aber vollständig von der thermisch induzierten Relaxation überdeckt. Dieser irreversible Effekt ist aufgrund der extremen Bedingungen, denen das Material bei der Laserablation ausgesetzt ist, besonders stark und wird bei Volumenmaterial nicht beobachtet.

Zur Beschreibung der thermisch induzierten Relaxation ist der Zugang über die Glasphysik allein also nicht ausreichend. Vielmehr scheinen auch chemische Veränderungen eine Rolle zu spielen. Die die Zunahme des Verlustmoduls bestimmende Aktivierungsenergie liegt im Bereich der für radikalische Polymerisation gefundenen Werte [117]. Es ist hier also nicht auszuschließen, dass durch die thermische Behandlung ein Kettenwachstum induziert wird, obwohl die gefundene Aktivierungsenergie natürlich nicht ausreicht, um existierende Bindungen zu brechen (Vgl. Kapitel 5.3). Nicht zu vernachlässigen ist der Anteil an reaktiven, kleinen Fragmenten, die seit der Deposition im Film eingeschlossen sind. Diese können radikalischen Charakter besitzen und somit zum Kettenwachstum beitragen. Die starke Zunahme der me- 
chanischen Härte lässt zudem auf einen steigenden Vernetzungsgrad schließen, s. d. hier zunächst nicht zwischen einem linearen Wachstum und dem Quervernetzen unterschiedlicher Makromoleküle unterschieden werden kann. Mit dem Ergebnis, dass der Speichermodul auch bei hohen Temperaturen nicht vollständig verschwindet, sondern das ausgelagerte Material einen duroplastischen Charakter zeigt, kann die Annahme, das zufällige Vernetzen sei die Ursache für die thermisch induzierte Zunahme des Speichermoduls, bevorzugt werden.

Die nachträgliche starke Veränderung des Polymers ist aber erst oberhalb der Glastemperatur möglich, was sich am Auftreten einer Schwellentemperatur in Abbildung 6.4 zeigt. Damit das mögliche Kettenwachstum oder die Vernetzung einsetzen kann, muss ausreichende Mobilität der Kettensegmente gegeben sein. Bei Raumtemperatur ist der Prozess durch die eingeschränkte Beweglichkeit der durch die UV-Strahlung gebildeten und noch im Material vorhandenen instabilen Moleküle und Fragmente, die Radikale bilden können, kinetisch gehemmt [131]. Ähnliches Verhalten zeigt sich auch für bei niedrigen Energiedichten deponierte PMMA-Schichten, deren Härte nach UV-Bestrahlung mehrere Monate zunehmen kann ${ }^{1}$ [132].

Durch thermische Auslagerung oberhalb der Glasübergangstemperatur wird dieser Prozess massiv beschleunigt [133], wobei die stärksten Veränderungen bei der höchsten angelegten Temperatur auftreten, s. d. die Veränderungen bei Temperaturen unterhalb der vorigen Auslagerungstemperatur zu vernachlässigen sind. Bei erneutem Aufheizen fallen somit keine signifikanten Unterschiede zur vorigen Kühlkurve auf. Eine Besonderheit stellen die Unterschiede der Heiz-Kühlkurven dar, die nicht mit AS-Moden bestimmt wurden. Diese wurden bereits oben auf Seite 96 diskutiert und werden zum Vergleich mit ähnlichen Ergebnissen am Poly(butyl methacrylat)e in Kapitel 7.2 auf Seite 131 erneut aufgegriffen.

Obwohl also anhand der Ergebnisse der Speichermodule der Prozess des zufälligen Quervernetzens priorisiert werden kann, kommen weitere Prozesse in Frage. Da diese Prozesse bei ähnlichen Temperaturen parallel stattfinden können, aber nicht vollständig ablaufen müssen, ist eine eindeutige Trennung schwierig. Mögliche Ordnungseinstellung oder Phasenseparation [134] kann bei den hier betrachteten Materialien aufgrund der Röntgenuntersuchungen sicher ausgeschlossen werden. Nicht auszuschließen hingegen ist der Masseverlust, sowohl durch thermische Degradation als auch durch das Abdampfen bereits im laserdeponierten Film vor-

\footnotetext{
${ }^{1}$ Dieser Prozess zeigt sich auch bei UV-gehärteten Lacken, hier ist die Härtezunahme während der Bestrahlung sehr schnell, es tritt jedoch anschließend eine sehr langsame Endhärtung auf.
} 
handener Fragmente, da eine zweifelsfreie Unterscheidung zwischen Zunahme des Speichermoduls und Abnahme der Masse nicht getroffen werden kann.

Als weitere mögliche Ursache kommt eine Reorientierung von Ketten bzw. Kettensegmenten in Frage. Die damit verbundene Kompaktierung des Materials führt zu einer Erhöhung der Energiedissipation im Material und führt somit zum Anstieg des Verlustmoduls. Die alleinige Erklärung über eine Zunahme der Dichte [135] reicht jedoch nicht aus und kann insbesondere die Zunahme der Dämpfung für hohe Temperaturen oberhalb der Glastemperatur nicht erklären: Durch Auslagerung wird nicht nur im Temperaturbereich des $\alpha$-Prozesses die Dämpfung verstärkt. Auch für Temperaturen zwischen dem $\alpha$-Maximum und der vorigen maximalen Auslagerungstemperatur ist die in den Folgemessungen gemessene Dämpfung erhöht. Gemäß dem Zeit-Temperatur-Superposition-Prinzip werden bei hohen Temperaturen zusätzliche langsamere Prozesse aktiviert [136]. Diese langsamen Prozesse können mit der Bewegung größerer Kettensegmente korreliert werden [137]. Durch die erhöhte Mobilität der Kettensegmente können dann thermisch aktiviert Verschlaufungen gelöst werden, die durch den Depositionsprozess im Material vorhanden sind. Durch dieses Ausheilen können größere Kettensegmente kollektiv an der Verformung teilnehmen und durch innere Reibung zu Energiedissipation bei hohen Temperaturen beitragen. Dies führt weiterhin dazu, dass auch kleinere Segmente mobiler werden und somit ebenfalls an der Verformung beteiligt werden, s. d. insgesamt die Dämpfung im gesamten Temperaturbereich ansteigen kann.

Im dielektrischen Verlust zeigt sich die thermisch induzierte Relaxation auf anderem Wege und kann in drei Bereiche aufgeteilt werden (Vgl. Kapitel 6.4.2.1-6.4.2.3). Während des ersten Messdurchgangs wird bereits bei niedrigen Temperaturen ein starker dielektrischer Verlust beobachtet, dessen Schwerpunkt mit steigender Temperatur zu höheren Frequenzen verschoben wird, ohne die Intensität zu verändern. Bereits beim folgenden Abkühlen ist der niederfrequente Bereich bei tieferen Temperaturen stark unterdrückt und die anhand der Frequenzverschiebung bestimmte Aktivierungsenergie um 50\% gesunken. Dies kann zum einen durch Abnahme der Anzahl effektiv zum dielektrischen Verlust beitragender Dipole etwa durch präferentielles Abdampfen von Seitengruppenfragmenten, zum anderen durch das Ausheilen von Verschlaufungen, wie auch schon im mechanischen Verlust beobachtet, erklärt werden. Da die $\beta$-Relaxation mit leichten Kippbewegungen in der Hauptkette korreliert ist [138], zeigt sich hier auch im dielektrischen Verlust der Einfluss des Fließprozesses. 
Die irreversible Abnahme der Aktivierungsenergie wird durch die Messungen der Gleichstromleitfähigkeit bestätigt. Wie in Abbildung 6.33 dargestellt, tritt hier eine Änderung der effektiven Aktivierungsenergie erst ab etwa $350 \mathrm{~K}$ auf. Dies ist ein Zeichen dafür, dass auch hier erhöhte Mobilität der Ketten notwendig ist, um den Leitungsprozess irreversibel zu verändern. Bis zu sehr hohen Auslagerungstemperaturen, die vermutlich zur Degradation des Materials führen, kann bei dem niederfrequenten Anteil, der mit dem $\beta$-Prozess verbunden werden kann, bulk-ähnliches Verhalten beobachtet werden. Die mit steigender Auslagerungstemperatur beobachtete Annäherung der Kurvenform an ein symmetrisches Debye-Maximum lässt auf eine Homogenisierung der Relaxationszeiten der beteiligten Dipole schließen [126, 127].

Nach Auslagerung bei Temperaturen von $500 \mathrm{~K}$ ist der Beitrag des $\beta$-Prozesses im niederfrequenten Bereich stark unterdrückt, stattdessen tritt hier nur noch das HF-Maximum auf. Hier ist von einer Degradation des Materials auszugehen, s. d. klassische $\beta$-Relaxation nicht mehr aktiviert werden kann. Eine Identifizierung des dem bleibenden HF-Maximum zugrundeliegenden mikroskopischen Prozesses gestaltet sich schwierig, da in der Literatur keine entsprechenden Beobachtungen gefunden werden konnten. Besonders das auftretende AntiArrhenius-Verhalten der Abhängigkeit der Maximumsfrequenz von der Temperatur scheint wenig einleuchtend, konnte aber in diversen Vergleichsmessungen reproduziert werden. Unzweifelhaft ist, dass es sich bei diesem Verhalten um eine PLD-spezifischen Eigenschaft handelt. Zusammen mit der im Vergleich zum Volumenmaterial stark erhöhten Gleichstromleitfähigkeit und den Ergebnissen der Massenfeinbestimmung könnten im Film durch die Deposition eingeschlossene Fragmente als Ursache für ionische Leitfähigkeit in Frage kommen, die die Ergebnisse der dielektrischen Spektroskopie beeinflussen. Eine genaue Klärung ist im Rahmen dieser Arbeit aber nicht möglich, s. d. auf eine weitere Interpretation, insbesondere auf die Diskussion des Anti-Arrhenius-Verhalten verzichtet wird.

Nicht zu vernachlässigen sind dabei auch Effekte, die an den Übergangskontakten auftreten

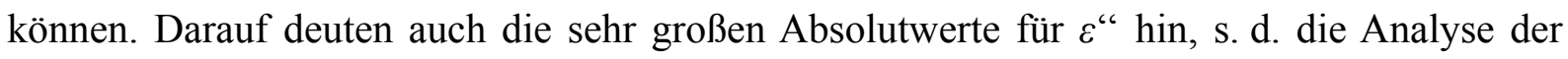
dielektrischen Eigenschaften evtl. ein komplexeres als das angenommene Ersatzschaltbild erfordert. 


\subsubsection{Verhalten relaxierter Proben}

Zum Vergleich der unterschiedlichen spektroskopischen Methoden werden im Folgenden Ergebnisse betrachtet, die an Proben mit vergleichbarem Relaxationszustand aufgenommen wurden. In Abbildung 6.36 ist die Dämpfungsdifferenz zwischen beschichteten und unbeschichteten Oszillatoren für die verschiedenen Schwingungsmoden des DPOs bzw. für die Biegeschwingung des PPXRs aufgetragen. Der DPO ist mit einer 1,4 $\mu \mathrm{m}$ dicken, das PPXR mit einer 2,8 $\mu \mathrm{m}$ dicken PEMA-Schicht beschichtet. Die Proben wurden vor der Messung bei $475 \mathrm{~K}$ ausgelagert.
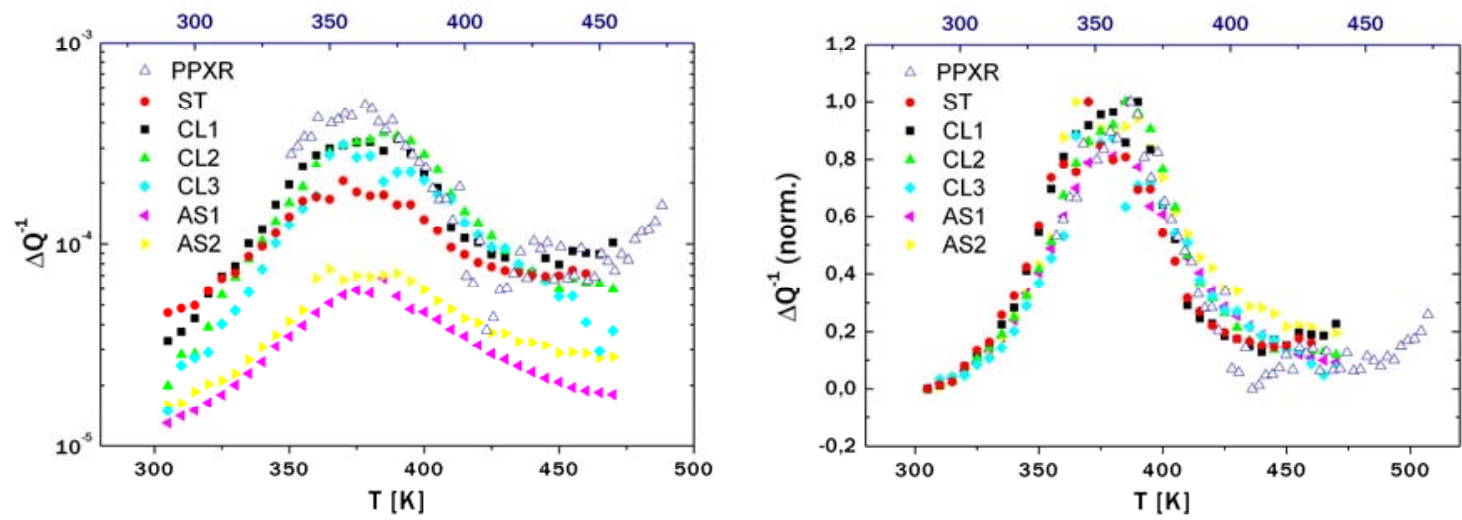

Abbildung 6.36: Dämpfungsdifferenz zwischen beschichteten und unbeschichteten Oszillatoren für die mit den verschiedenen Methoden zugänglichen Schwingungsmoden, links sind die Absolutwerte in logarithmischer Skala aufgetragen, rechts in linearer Skala die normierten Werte. Die vollen Symbole repräsentieren die Messungen am AllMode-DPO $(1,4 \mu \mathrm{m})$, die leeren Dreiecke zeigen die mit dem PPXR $(2,8 \mu \mathrm{m})$ bei $1.700 \mathrm{~Hz}$ bestimmten Werte. Die Temperaturskala für die Ergebnisse der PPXR-Messung ist um $15 \mathrm{~K}$ verschoben, was auf apparative Unterschiede zurückzuführen ist.

Die zu den mit dem PPXR aufgenommenen Messwerten gehörende Temperaturskala am oberen Rand des Graphen ist um $15 \mathrm{~K}$ nach rechts verschoben. Diese Verschiebung ist auf apparative Unterschiede zurückzuführen und konnte bereits von Scharf beobachtet werden [16].

Im linken Teil der Abbildung sind die Absolutwerte der Dämpfungsdifferenz in logarithmischer Skala dargestellt. Hier zeichnen sich die Ergebnisse, die mit den beiden Asymmetrischen Torsionsmoden AS1 und AS2 bestimmt wurden, durch sehr geringe Dämpfung aus. Die Dämpfungswerte aller weiteren Moden liegen weit oberhalb dieser Werte. Signifikante Unterschiede in Form und Position der Maxima sind in dieser Auftragung hingegen nur schwer auszumachen.

Auch in der Darstellung der normierten Dämpfungswerte im rechten Teil von Abbildung 6.36 lassen sich kaum Unterschiede in der Form der einzelnen Dämpfungsmaxima feststellen. Die 
mit dem PPXR gemessenen Werte zeigen einen mit den DPO-Kurven vergleichbaren Kurvenverlauf. Unterschiede zwischen den Messdaten des DPOs und des PPXR, wie sie von Scharf für PMMA gefunden wurden [16], treten hier nicht auf, können aber auch nicht gänzlich ausgeschlossen werden, da die PPXR-Messung nicht zuverlässig bis in den Bereich weit unter der Glasübergangstemperatur durchgeführt werden konnte. Anhand der in Kapitel 7 folgenden Ergebnisse an PMMA und PBMA wird jedoch gezeigt werden, dass auch die Niedertemperaturflanken der Dämpfungen, die mit den unterschiedlichen Methoden aufgenommen wurden keine signifikanten Unterschiede aufweisen.

Für Proben, die sich nach Auslagerung schließlich in einem klar definierten und erholten, insbesondere auch vergleichbaren Relaxationszustand befinden, werden also mit den hier untersuchten Methoden unabhängig von Messfrequenz und Art der Anregung (Biege- oder Torsionsschwingung) analoge Verlustspektren gefunden, die die Charakteristik eines reinen $\alpha$-Prozesses aufweisen. Dabei kann hier keine eindeutige Abhängigkeit der Maximumstemperatur, bzw. der Glasübergangstemperatur von der Messfrequenz gefunden werden, wie schon in Abbildung 6.22 dargestellt. Bedingt durch den hohen Fehler mit dem die Temperatur des Maximimus behaftet ist, kann diese Abhängigkeit hier aber auch nicht ausgeschlossen werden. Dennoch scheint die Abhängigkeit der Fließprozesse von der Messfrequenz im engen Messfenster von $5 \mathrm{kHz}$ hier als zu gering, um aufgelöst zu werden zu können.

Die beim ersten Vergleich der Messungen von DPO und PPXR in Kapitel 6.2 aufgetretenen Abweichungen im Hochtemperaturverhalten können hier nicht verifiziert werden. Die Dämpfung bei hohen Temperaturen steigt für die hier gezeigten Messungen weder für die vom Elastizitätsmodul noch für die vom Schermodul dominierten an. Das in Kapitel 6.2 gefundene abweichende Verhalten scheint also experimentellen Ursprungs zu sein. Möglich ist ein Einfluss der Umgebungsluft auf das Hochtemperaturverhalten, da experimentell bedingt für die Messungen in der ex-situ Anlage das Vakuum gebrochen werden musste. Wahrscheinlicher ist aber der Einfluss der unterschiedlichen Ofengeometrien und ein eventuelles Überheizen der Schicht im Strahlungsofen der ex-situ Anlage, das zu einem verstärkten duroplastischen Charakter der Schicht und somit zu erhöhter Dämpfung bei hohen Temperaturen führen würde.

Deutliche Unterschiede zeigen sich im Vergleich mit den Ergebnissen der dielektrischen Spektroskopie. In Abbildung 6.37 ist der temperaturabhängige dielektrische Verlust einer mit 400 Pulsen hergestellten PEMA-Schicht dargestellt. Die unterschiedlichen Kurven repräsentieren die Daten, die bei unterschiedlichen Frequenzen, ähnlich denen der mechanischen Mes- 
sungen in Abbildung 6.36, aufgenommen wurden. Das in Abbildung 6.36 dargestellte, in seiner Position nahezu frequenzunabhängige, Maximum der mechanischen Dämpfung des $\alpha$-Prozesses findet hier keine Entsprechung. Stattdessen ist bei geringeren Temperaturen ein Maximum im Verlust zu erkennen, das auf den in der dielektrischen Spektroskopie dominierenden $\beta$-Prozess zurückzuführen ist. Dieser $\beta$-Prozess zeigt im Gegensatz zum mechanisch gemessenen viskosen Fließprozess eine starke Abhängigkeit der Temperatur des maximalen Verlustes von der Messfrequenz. Mit steigender Frequenz wird die Lage des Verlustmaximums zu höheren Temperaturen verschoben.
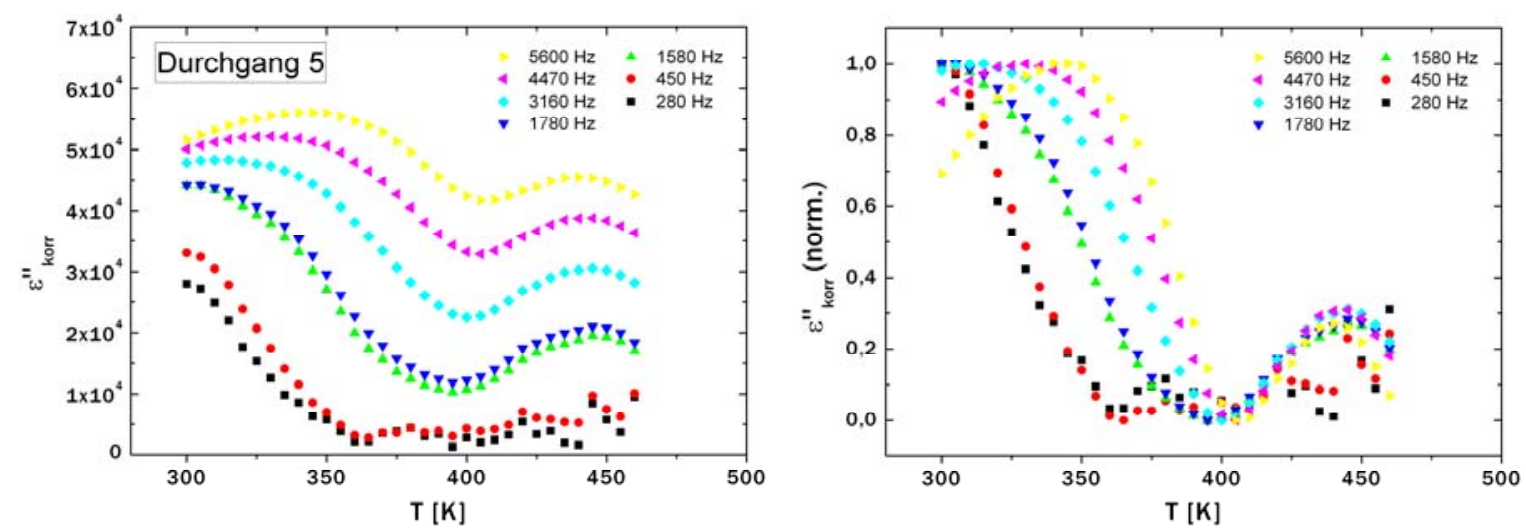

Abbildung 6.37: Temperaturabhängiger dielektrischer Verlust einer mit 400 Pulsen laserdeponierten PEMA-Probe, aufgetragen bei verschiedenen Frequenzen, die auch mit den Methoden der mechanischen Spektroskopie zugänglich sind. Im linken Graph sind die Absolutwerte aufgetragen, rechts sind die Daten normiert dargestellt.

Während die Position der Maximumsfrequenzen und somit die typischen Relaxationszeiten den Literaturdaten von bulk-PEMA entsprechen, sind die anhand der Arrhenius-Auswertung in Abbildung 6.29 gefundenen Aktivierungsenergien von 0,21 eV etwa einen Faktor 3 bis 4 geringer als die entsprechenden Werte in der Literatur [127, 128, 139-142]. Eine mögliche Ursache ist wiederum in der Modifikation des Polymers während der Laserbehandlung zu finden. Insbesondere der mögliche Verlust vereinzelter Ester-Seitengruppen, der z.B. bei Laserbestrahlung von PMMA auftritt [12], hat einen starken Einfluss auf die $\beta$-Relaxation. Ein homogener Verlust der Seitengruppen, der zur Bildung von alternierenden Einfach- und Doppelbindungen im Backbone und somit zu konjugierten Bindungen, ähnlich wie im Polyacetylen, führen würde [7], ist aber unwahrscheinlich, auch wenn dadurch die erhöhte Gleichstromleitfähigkeit teilweise erklärt werden könnte. Wichtiger scheint der Einfluss im Polymer eingeschlossener kleiner radikaler Fragmente, die als zusätzliche Ladungsträger auftreten können. 
Bei Temperaturen von etwa $440 \mathrm{~K}$ tritt in Abbildung 6.37 ein zweites, frequenzunabhängiges Maximum auf. Die Interpretation dieses Maximums als Ergebnis des aus der mechanischen Spektroskopie bekannten $\alpha$-Prozesses scheidet beim Vergleich der Temperaturen des maximalen Verlustes aus. Vielmehr scheint es seine Ursache in Polarisationsprozessen in der glatten Komponente des PEMAs zu haben. Hier ist erneut zu betonen, dass die hier untersuchten laserdeponierten Polymere zweikomponentige Materialien sind. Während in der mechanischen Spektroskopie sicher der Einfluss der bulk-ähnlichen Tröpfchenkomponente überwiegt [16], ist insbesondere in der dielektrischen Spektroskopie der Einfluss der glatten Komponente, die sich durch eine starke Modifikation im Vergleich zu Ausgangsmaterial und insbesondere starke Vernetzung auszeichnet, nicht zu vernachlässigen. 


\section{Vergleich mit Poly(methyl methacrylat) und Poly(butyl methacrylat)}

Nachdem im vorigen Kapitel die Eigenschaften des Poly(ethyl methacrylat)s eingehend und auch quantitativ dargelegt wurden, sollen im folgenden Kapitel zwei weitere Homologe der Poly(alkyl methacrylat)e untersucht werden: PMMA und PBMA.

Der Schwerpunkt liegt auf der inneren Dynamik der polymeren Systeme, die wiederum sowohl mit dem DPO, als auch mit dem PPXR untersucht werden kann. Dabei soll die Frage geklärt werden, in wie weit die Dynamik, insbesondere die dem Fließprozess zugrundeliegende, durch die unterschiedlichen Seitengruppen bestimmt wird, bzw. in wie weit alle untersuchten Homologen universelles Verhalten zeigen. Von besonderem Interesse ist zusätzlich wieder die Untersuchung der bereits bei Auslagerungsversuchen am PEMA gefundenen thermisch induzierten Relaxation.

Zunächst werden beginnend mit den Untersuchungen die Ergebnisse der beiden Materialien vorgestellt, wobei im Falle des PMMAs zusätzlich die Abhängigkeit der Dynamik von der Schichtdicke beschrieben wird. Durch den Vergleich der an relaxierten Systemen gefundenen Verlustspektren mit Literaturdaten aus [16] für das PMMA und eigenen Messungen im Falle des PBMAs liegt dann zusammen mit den aus Kapitel 6 bekannten Charakteristiken des PEMAs eine umfassende Beschreibung vor, mit der es möglich ist, eindeutig zwischen materialspezifischen und methodisch bedingten Besonderheiten unterscheiden zu können.

\subsection{Mechanische Dämpfung in Poly(methyl methacrylat)}

Die Ergebnisse zum PMMA wurden mit dem in Kapitel 4.1.3 beschriebenen All-Mode-DPO gewonnen. Hier wurde die in-situ Fähigkeit ausgenutzt, um neben der thermisch induzierten Relaxation zunächst die Abhängigkeit der Filmeigenschaften von der Schichtdicke zu unter- 
suchen. Da bei geringen Dicken und insbesondere bei noch nicht ausgelagerten Schichten die durch die Schicht verursachte Dämpfung sehr gering ist, ist hier die ausgezeichnete Empfindlichkeit der Torsionsmoden des DPOs notwendig, um hinreichend hohe Signal-RauschVerhältnisse zu erreichen. Bei gewöhnlichen Oszillatoren hingegen, wie auch beim PPXR oder den Biegeschwingungen des DPOs wäre keine signifikante Dämpfungsdifferenz zwischen beschichtetem und unbeschichtetem Oszillator aufzulösen.

Abbildung 7.1 zeigt die während Temperaturbehandlung sukzessiv aufgenommene Dämpfungsdifferenz für eine mit 500 Laserpulsen bei Raumtemperatur hergestellte PMMA-Schicht. Aus Vergleichsmessungen der Depositionsrate bestimmt sich die Schichtdicke zu etwa $200 \mathrm{~nm}$.
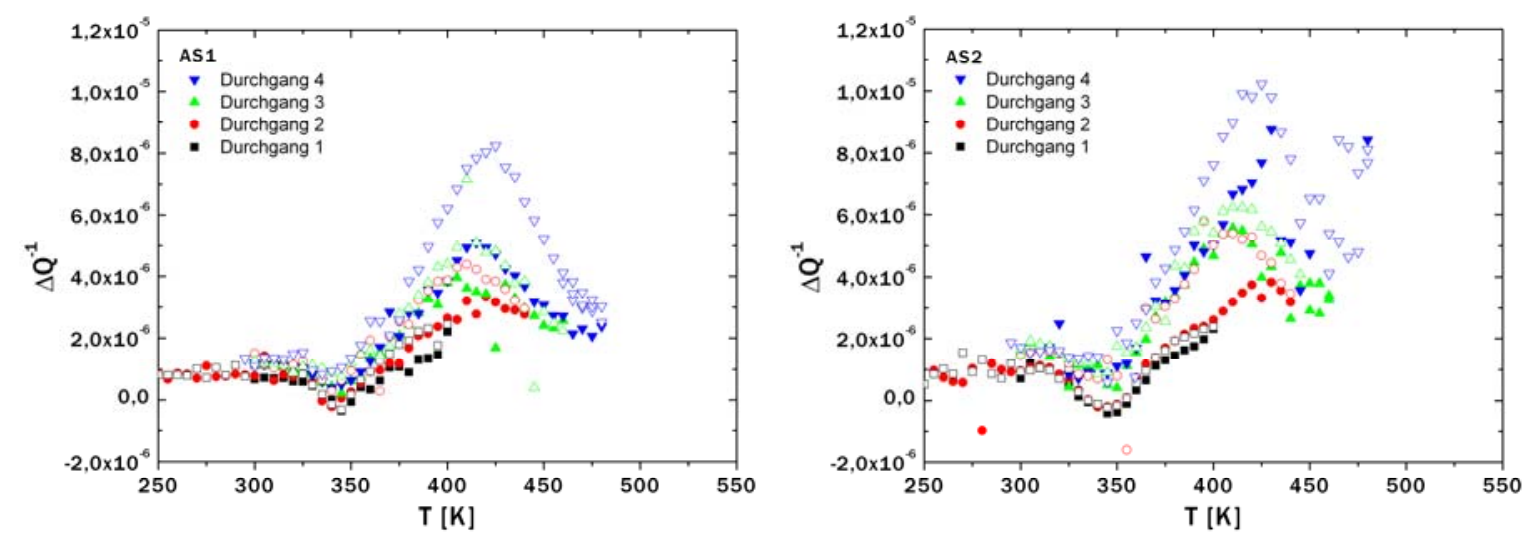

Abbildung 7.1: Abhängigkeit der Dämpfungsdifferenz von der Auslagerung für eine mit 500 Pulsen PMMA hergestellten Dünnschicht, gemessen mit der AS1-Mode bei 4.660 Hz und der AS2-Mode bei $5.340 \mathrm{~Hz}$. Auch bei dieser sehr dünnen Schicht lässt sich der Einfluss der thermisch induzierten Relaxation erkennen.

Im linken Teil der Abbildung ist die mit der AS1-Mode bei $4.660 \mathrm{~Hz}$ bestimmte Dämpfungsdifferenz aufgetragen. Die schwarzen vollen Quadrate zeigen die Daten der Neukurve nach Deposition bei Raumtemperatur. Die Dämpfung läuft hier zunächst konstant bei sehr geringen Werten unterhalb $1 \cdot 10^{-6}$ und zeigt dann bei etwa $350 \mathrm{~K}$ ein Minimum. Dieses ist auf Artefakte, die während der Leermessung auftraten, zurückzuführen. Das vermutlich leicht kontaminierte „leere“ DPO zeigte in diesem Temperaturbereich ein schwaches Maximum, welches sich nach der Differenzbildung mit den geringen Werten der Messung am beschichteten Paddel als Minimum darstellt. Ab etwa $350 \mathrm{~K}$ steigt die Dämpfung an, bis bei $400 \mathrm{~K}$ wieder abgekühlt wird. Die Daten dieser Kühlmessung laufen knapp oberhalb der Heizmessung zurück. Die thermisch induzierte Relaxation hatte bis zu Temperaturen von $400 \mathrm{~K}$ also noch keinen großen Einfluss auf die Dämpfung im Polymer. Als notwendige Bedingung für irreversible Veränderungen kommt eine erhöhte Mobilität von Kettensegmenten in Frage. Wie auch beim 
PEMA kann hier also eine Schwellentemperatur angenommen werden, unter der keine ausreichende Mobilität auftritt. Hier kann abgeschätzt werden, dass diese Temperatur für das PMMA bei um etwa $40 \mathrm{~K}$ höheren Werten liegt als im Falle des PEMAs (Vgl. Kapitel 6.1). Die in diesem Kühldurchlauf zusätzlich unterhalb von Raumtemperatur gemessene Dämpfung liegt auf dem niedrigen Niveau der Raumtemperatur-Daten. Insbesondere zeigen sich hier keine weiteren ausgezeichneten Dämpfungsprozesse.

Die nächste Heizmessung (rote Punkte) reproduziert wieder die Daten der vorigen Kühlmessung. Bei etwa $415 \mathrm{~K}$ erreicht die Dämpfung ein Maximum, das wie auch beim PEMA dem $\alpha$-Prozess zugeordnet werden kann. Nach Erreichen der maximalen Temperatur dieses Messdurchgangs von $440 \mathrm{~K}$ hat dieses Maximum in der Kühl- im Vergleich zur Heizmessung stark an Intensität gewonnen. Die thermisch induzierte Relaxation führt nun analog zu den an PEMA gefundenen Ergebnissen in Kapitel 6 zu einer starken Zunahme der Energiedissipation im PMMA. Die folgende Heizmessung des dritten Messzyklus ist leider im Temperaturbereich des Maximums verrauscht, entspricht aber im groben Verlauf den bekannten Ergebnissen. Auch die nächste Kühlmessung liegt erwartungsgemäß bei höheren Werten. Ebenfalls wie erwartet reproduziert die letzte Heizmessung (blaue Dreiecke) die Daten der vorigen Kühlmessung. Die letzte Kühlmessung nach Auslagerung bei $480 \mathrm{~K}$ zeigt dann mit stark gestiegener Dämpfung im Bereich des Maximums das für einen weitgehend relaxierten Zustand charakteristische Verhalten.

Im rechten Teil der Abbildung sind die entsprechenden Daten, die mit der AS2-Mode bei 5.340 Hz gemessen wurden, dargestellt. Das zuvor anhand der AS1-Mode beobachtete Verhalten wird weitgehend reproduziert. Aufgrund der ausgezeichneten Güte der AS2-Schwingung lassen sich hier in der Neukurve zwei Stufen in der Tieftemperaturflanke des $\alpha$-Maximums erkennen: Die Dämpfungsdifferenz im zweiten Heizdurchgang (rote Kurve) zeigt bei $400 \mathrm{~K}$ einen weiteren Knickpunkt, der auf den ab $400 \mathrm{~K}$ verstärkten Einfluss irreversibler Veränderung im Material zurückzuführen ist. Dieser Knickpunkt markiert die oben diskutierte Schwelltemperatur, ab der ausreichende Mobilität von Kettensegmenten das Einsetzen der thermisch induzierten Relaxationsprozesse erlaubt. Die weiteren Messungen zeigen den bekannten Verlauf.

Nach Interpolation der durch das Minimum gestörten Werte lässt sich aus den Temperaturen des ersten Knickpunkts für alle Dämpfungskurven der AS-Moden die Glasübergangstemperatur zu $355 \pm 7 \mathrm{~K}$ ablesen. Diese ist damit etwa $10 \mathrm{~K}$ geringer als die an vergleichbaren, aber 
mit $t>1 \mu \mathrm{m}$ bedeutend dickeren Proben gemessene Temperatur [16, 97]. Da diese Werte jedoch mit verschiedenen experimentellen Methoden gemessen wurden, kann der apparative Fehler in der Bestimmung der absoluten Temperatur hier überwiegen. Dieser apparative Fehler kann im Folgenden durch die Verwendung der in-situ Methode ausgeschlossen werden.

Zur weiteren Untersuchung dieser Schichtdickenabhängigkeit wurde das DPO nach den ersten vier Messdurchgängen bei Raumtemperatur sukzessive weiter beschichtet und jeweils erneut dessen Dämpfung vermessen. Die in Abbildung 7.2 und Abbildung 7.3 dargestellten Graphen zeigen die dabei gewonnenen Ergebnisse für den mit 500, 1.000, 2.000 und 4.000 Pulsen PMMA beschichteten Oszillator. Die Schichten sind vor der Messung bei $480 \mathrm{~K}$ (für die mit 500 Pulsen hergestellte Schicht) bzw. $500 \mathrm{~K}$ (für alle weiteren Schichten) ausgelagert worden, befinden sich also in einem vergleichbaren Relaxationszustand. In Abbildung 7.2 ist klar die vom PEMA bekannte Zunahme der Dämpfungsintensität mit steigender Schichtdicke zu erkennen. Während dabei die Dämpfung der dünnen Schichten nur im Bereich des $\alpha$-Maximums auftritt, zeigen die Proben, die mit mindestens 2.000 Pulsen (entspricht etwa $800 \mathrm{~nm}$ Schichtdicke) hergestellt wurden, signifikante Dämpfungsbeiträge auch unterhalb von $350 \mathrm{~K}$ bzw. oberhalb von $475 \mathrm{~K}$. Insbesondere fällt der lineare Anstieg bei tiefen Temperaturen auf, der im Falle des PEMAs in dieser Stärke nicht auftritt. Da im untersuchten Frequenzbereich $\alpha$ - und $\beta$-Prozesse bei ähnlichen Temperaturen auftreten [143], kann diese Flanke vermutlich auf den Einfluss des $\beta$-Prozesses zurück geführt werden [144], der sich wenn auch abgeschwächt, in der mechanischen Messung wider spiegelt.
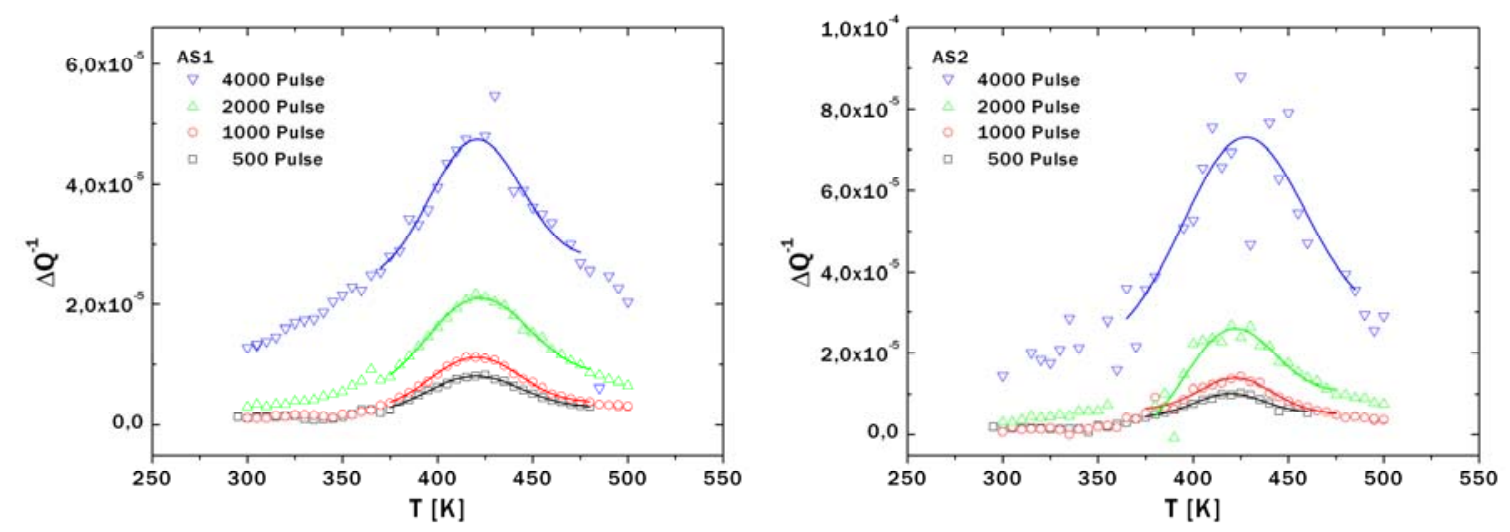

Abbildung 7.2: Schichtdickenabhängigkeit der Dämpfung einer PMMA-Schicht gemessen mit der AS1-Mode bei 4.660 Hz (links) und der AS2-Mode bei $5.340 \mathrm{~Hz}$ (rechts). Zusätzlich eingetragen sind numerische Anpassungen (Summe aus Gerade und Gauß-Funktion), aus der die Temperaturen maximaler Dämpfung bestimmt werden können.

Für sehr große Schichtdicken ist die mit der AS2-Mode aufgenommene Messung stark verrauscht. Hier ist der Dämpfungseffekt in der Schicht so groß, dass die Schwingungsamplitude 
der aufgezeichneten Ringdowns sehr schnell abfällt und somit nur wenige Messpunkte pro Ringdown aufgenommen werden können, s. d. der apparative Fehler zu- und die Qualität der Messung abnimmt. Zur Untersuchung der beim PMMA mit Schichtdicken über $2 \mu \mathrm{m}$ auftretenden starken Dämpfung ist die empfindliche AS2-Mode also nur noch wenig geeignet.

Aus den Daten der AS1-Mode lässt sich durch das Anlegen zweier Regressionsgeraden an den Bereich vor dem Maximum und die aufsteigende Flanke des Maximums die Glasübergangstemperatur gut bestimmen. Es ergibt sich mit steigender Schichtdicke systematisch eine leichte Zunahme der Glasübergangstemperatur von 355 auf $373 \mathrm{~K}$, die trotz des systematischen Fehlers der Ausgleichsgeraden und ihres Schnittpunkts von $7 \mathrm{~K}$ als signifikant angesehen werden kann. Diese Abhängigkeit von der Schichtdicke ist für $200 \mathrm{~nm}$ dicke Proben ungewöhnlich. Der Vergleich mit Literaturdaten liefert eine signifikante Abhängigkeit der Glasübergangstemperatur erst bei Schichten unter $100 \mathrm{~nm}$ Dicke [145, 146]. Als Ursache für diese Diskrepanz kommt zunächst die Ungenauigkeit der bestimmten Filmdicke in Frage. Dies wird auf S. 127 in Zusammenhang mit den Ergebnissen der anderen Moden diskutiert. Andererseits können die speziellen Eigenschaften der laserdeponierten Schichten, insbesondere der zweikomponentige Charakter, dazu führen, dass bereits bei vergleichsweise großen Schichtdicken die erhöhte Mobilität der Kettensegmente an der Filmoberfläche ausreicht, um die Glasübergangstemperatur des gesamten Systems zu verringern. Für die Daten der AS2-Mode ist die Bestimmung der Onset-Temperaturen nicht möglich, s. d. hier nur die Maximumstemperaturen zugänglich sind. Diese sind unten in Abbildung 7.4 dargestellt.

Abbildung 7.3 zeigt die Ergebnisse, die mit der ST-Mode bei $460 \mathrm{~Hz}$ und der CL2Biegeschwingung bei $1.700 \mathrm{~Hz}$ gewonnen wurden. Während bei der ST-Mode auch für geringe Schichtdicken ein Dämpfungsmaximum angedeutet ist, reicht die Güte bei der CL2Biegeschwingungsmode hier nicht aus, um Effekte bei Schichten, die mit weniger als 4.000 Pulsen hergestellt wurden, aufzulösen, s. d. hier auf die numerische Anpassung verzichtet wurde. Die stark dämpfende Probe, die mit 4.000 Pulsen hergestellt wurde, zeigt mit dem Dämpfungsmaximum bei etwa $410 \mathrm{~K}$ ein zu den weiteren Messungen analoges Verhalten. Der starke Anstieg bei niedrigen Temperaturen unter $370 \mathrm{~K}$ ist auf eine Störung der Messung durch Annäherung der CL2-Resonanzfrequenz an die Frequenz der Schwingung der Turbomolekularpumpen zurückzuführen und zu vernachlässigen. 

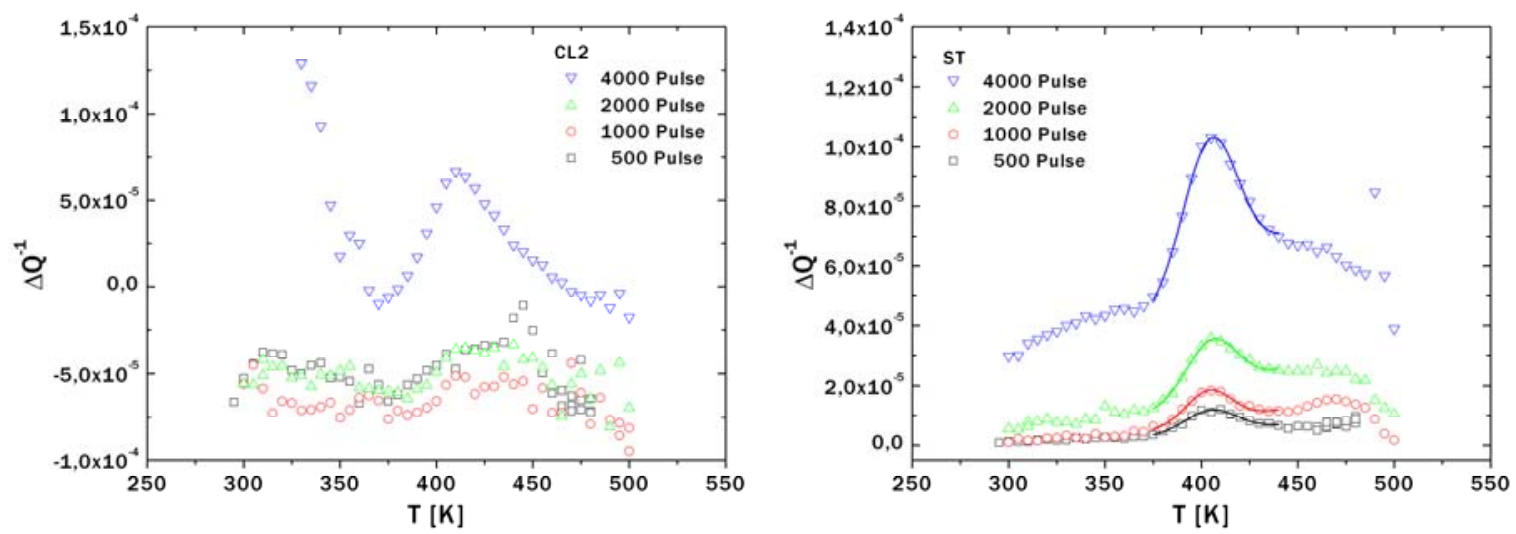

Abbildung 7.3: Schichtdickenabhängige Dämpfung einer PMMA-Schicht gemessen mit der CL2-Mode bei $1.700 \mathrm{~Hz}$ (links) und der ST-Mode bei $460 \mathrm{~Hz}$ (rechts). Für die ST-Mode sind zusätzlich numerische Anpassungen (Summe aus Gerade und Gauß-Funktion) eingetragen, aus der die Temperaturen maximaler Dämpfung bestimmt werden können. Für die CL2-Mode sind solche Anpassungen nicht möglich.

Die mittels der ST-Mode gewonnenen Ergebnisse entsprechen weitestgehend denen der ASModen. Bereits bei geringen Schichtdicken tritt ein zunächst schwaches $\alpha$-Maximum auf, dessen Intensität mit steigender Schichtdicke zunimmt. Im Vergleich zu den Verlustspektren der vorigen Moden fallen aber auch Unterschiede auf: Zum einen erstreckt sich das Maximum über einen engeren Temperaturbereich, was gleichzeitig dazu führt, dass die Temperatur der maximalen Dämpfung um $20 \mathrm{~K}$ verringert ist. Zum anderen ist hier entgegengesetzt zu den Ergebnissen der AS-Moden auch schon bei geringen Schichtdicken (ab 1.000 Pulse) auch oberhalb von $450 \mathrm{~K}$ ein von Null verschiedener Dämpfungsbeitrag der Schicht zu messen. Für die dünnen Proben (rote und grüne Punkte) verschwindet die Dämpfung bei hohen Temperaturen wieder, s. d. sich für die mit 1.000 bzw. 2.000 Pulsen hergestellten Proben bei etwa $465 \mathrm{~K}$ jeweils ein zweites Maximum ergibt. Dieses ist systematisch auch in den aus Gründen der Übersichtlichkeit hier nicht gezeigten Zwischenmessungen (sowohl Heiz- als auch Kühlmessungen) mit verschiedenen Relaxationszuständen zu finden. Für große Schichtdicken (blaue Kurve) ist schließlich nur, wie auch bei den AS-Moden eine Hochtemperaturflanke zu erkennen. Dieses zweite Maximum kann auch in den mit den AS-Moden durchgeführten Messungen vorhanden sein, wird dort aber durch das Hauptmaximum überdeckt und kann somit nicht aufgelöst werden. Aufgrund der geringen Breite des Hauptmaximums und der Absenkung der Maximumstemperatur können im Falle der ST-Mode beide Prozesse getrennt werden. Als Ursache für die zusätzliche Dämpfung bei hohen Temperaturen kommt wie auch im Falle des PEMAs der durch die thermische Auslagerung gestiegene Vernetzungsgrad in Frage, der dazu führt, dass das Polymer auch im Temperaturbereich oberhalb des Glasübergangs endliche Viskosität besitzt und somit Energie dissipiert werden kann. 

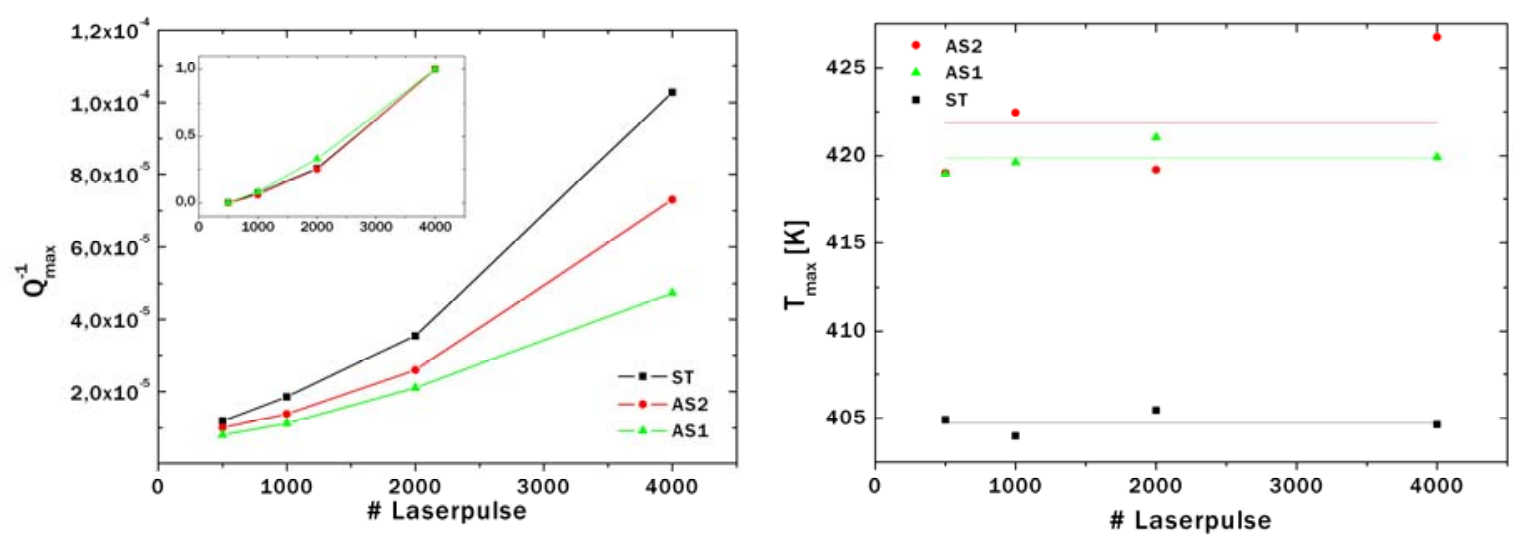

Abbildung 7.4: Links: Schichtdickenabhängigkeit der maximalen Dämpfung, im Inset in normierter Form aufgetragen. Rechts: Temperatur mit maximaler Dämpfung in Abhängigkeit von Anzahl der zur Deposition benutzten Laserpulse. Die Werte sind bestimmt aus numerischen Anpassungen an die Dämpfungskurven für die AS1, AS2 und STMode.

In Abbildung 7.4 sind die anhand der numerischen Anpassungen an die Dämpfungskurven bestimmten Ergebnisse zur Schichtdickenabhängigkeit zusammengefasst. Im linken Teil der Abbildung ist die maximale Dämpfungsdifferenz in Abhängigkeit von der Zahl der zur Deposition verwendeten Laserpulse für die ST-, die AS2- und die AS1-Mode aufgetragen. Im Inset sind die Daten in normierter Form dargestellt. Hier zeigt sich, dass die Zunahme der Dämpfungsintensität für alle Moden ähnlich mit der deponierten Masse skaliert, hier also keine Moden-, bzw. Frequenzabhängigkeit vorliegt. Die Zunahme der maximalen Dämpfung ist jedoch nicht linear mit der Zahl der verwendeten Laserpulse, wie es für Filme mit vergleichbarem Relaxationszustand zu erwarten wäre [16]. Als mögliche Ursache kommt zunächst, wie auch bei der Diskussion der Schichtdickenabhängigkeit der Glasübergangstemperatur eine nicht konstante Depositionsrate in Frage. Obwohl die verwendeten Targets vor Benutzung „eingeschossen“ wurden, können Inkubationseffekte (Vgl. Kapitel 5.3) nicht vollständig ausgeschlossen werden. Da diese allerdings nach wenigen zehn Pulsen abgeschlossen sind und außerdem zu einer verringerten Depositionsrate für geringe Pulszahlen führen, hier also entgegengesetzte Tendenz zeigen, müssen andere Effekte verantwortlich sein. Möglich ist der oben diskutierte Einfluss der $\beta$-Relaxation. Eine Überlagerung zweier Prozesse kann dann dazu führen, dass kein einfacher linearer Zusammenhang zwischen Schichtdicke und maximaler Dämpfung besteht.

Im rechten Teil von Abbildung 7.4 sind die über die numerischen Anpassungen bestimmten Temperaturen des Maximums für drei der untersuchten Schwingungsmoden dargestellt. Hier zeigt sich - entgegengesetzt zu den obigen Ergebnissen der Glasübergangstemperatur - für alle Moden keine signifikante Abhängigkeit von der Schichtdicke. Stattdessen ist hier die Abhän- 
gigkeit der Lage des Maximum von der Messfrequenz ersichtlich: Für die langsame ST-Mode tritt das Maximum bei um etwa $20 \mathrm{~K}$ verringerten Temperaturen auf. Die frequenzabhängigen relativen Verschiebungen der Maximumstemperaturen, die Scharf mittels PPXR-Messungen an PMMA bestimmt hat, können also bestätigt werden [16]. Ebenda wurde eine Verschiebung von 396 auf $409 \mathrm{~K}$ bei Messfrequenzen zwischen 1.600 und $7.500 \mathrm{~Hz}$ festgestellt.

Zur Demonstration der Modenabhängigkeit sind in Abbildung 7.5 die mit verschiedenen Moden aufgenommenen Dämpfungskurven vergleichbarer PMMA-Schichten aufgetragen.
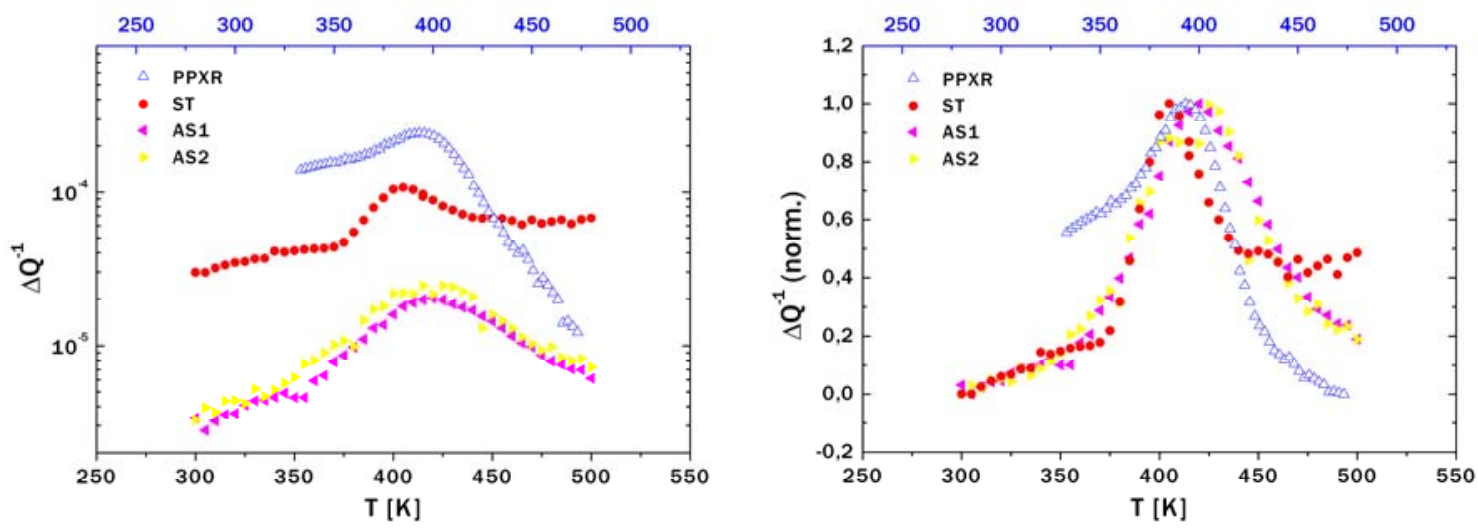

Abbildung 7.5: Vergleich der Dämpfungskurven von PMMA-Schichten mit Schichtdicken über 1,5 $\mu \mathrm{m}$ für die unterschiedlichen Schwingungsmoden des DPOs, links in logarithmierter rechts in normierter Auftragung. Zusätzlich eingetragen sind die Daten einer PPXR-Messung bei $2.700 \mathrm{~Hz}$ an einer vergleichbaren PMMA Schicht ([16]).

Im linken Teil der Abbildung sind die Absolutwerte der Dämpfungsdifferenz in logarithmischer Skala dargestellt. Erneut zeichnen sich die Ergebnisse, die mit den beiden Asymmetrischen Torsionsmoden AS1 und AS2 bestimmt wurden, durch sehr geringe Dämpfung aus. Die absoluten Dämpfungswerte der ST-Mode liegen weit oberhalb dieser Werte. Auch die PPXRMessung aus [16] zeigt im Maximumsbereich eine höhere Dämpfung, fällt für hohe Temperaturen aber steil ab. Wie bereits bei den vergleichenden Auftragungen in Kapitel 6.6 ist auch hier die PPXR-Temperaturskala (oben) im Vergleich zur DPO-Temperaturskala (unten) verschoben, hier um $20 \mathrm{~K}$.

In der normierten Darstellung der Dämpfungswerte im rechten Teil von Abbildung 7.5 lassen sich weitere Unterschiede beobachten: Während die Kurvenformen, die mit den AS-Moden bestimmt wurden, bis auf die Verschiebung der Temperaturskala zu höheren Werten qualitativ denen des PEMAs entsprechen (Vgl. Abbildung 6.36), zeigt die Kurve der ST-Mode mit einer verringerten Breite und der oben diskutierten Verschiebung des Maximums zu geringeren Werten signifikante Abweichungen. Für hohe Temperaturen oberhalb des Maximums fällt die 
Dämpfungsintensität hier nicht stetig ab, sondern weist auch oberhalb von $450 \mathrm{~K}$ noch mehr als $40 \%$ der minimalen Dämpfung auf, was vermutlich auf den oben diskutierten Einfluss von Vernetzung zurückzuführen ist.

Die von Scharf mit dem PPXR bestimmten Daten aus [16] (blaue Dreiecke) zeigen weitere Abweichungen. Hier ist die Dämpfung im Bereich des linearen Anstieg vor der Glasübergangstemperatur bedeutend stärker ausgeprägt als bei den DPO-Messungen, wohingegen der Dämpfungsbeitrag für hohe Temperaturen nahezu vollständig verschwindet. Scharf führte die Unterschiede in diesem qualitativen Verlauf der Kurven auf methodische Unterschiede zwischen DPO und PPXR zurück: Während, bedingt durch die unterschiedlichen Arten der Schwingung, mit den Torsionsmoden des DPOs hauptsächlich der Schermodul bestimmt wird, wird mit der Biegeschwingung des PPXR überwiegend der Elastizitätsmodul gemessen. Da beide Größen über die Poissonzahl miteinander verknüpft sind, bleibt schließlich die Temperaturabhängigkeit ihres jeweiligen Imaginärteils als mögliche Ursache für die starken Unterschiede [16]. Diese signifikanten Unterschiede konnten hier mit eigenen Messungen nicht verifiziert werden, da die Messungen der hier betrachteten CL2-Biegeschwingung im fraglichen Niedertemperaturbereich eine Störung aufweisen. Die Ergebnisse, die mit den weiteren Homologen (Vgl. Kapitel 6.6 und 7.1) erzielt wurden, zeigen aber keine Analogie zu diesem Verhalten. Sowohl die Torsions- als auch die Biegeschwingungen liefern hier symmetrische Maxima und einen weiteren Anstieg der Dämpfung mit steigender Temperatur oberhalb des Maximums, in jedem Fall aber eine von Null verschiedene Dämpfung auch bei hohen Temperaturen. Dies ist, wie oben diskutiert, vermutlich auf vernetztes Material zurückzuführen, das auch bei hohen Temperaturen zu einem duroplastischen Verhalten führt.

Scharf konnte solches Verhalten nur bei PMMA-Filmen finden, die bei erhöhten SubstratTemperaturen von $500 \mathrm{~K}$ laserdeponiert wurden und führte es auf temperaturunterstützte Quervernetzung von Makromolekülen während des Depositionsprozesses zurück [16]. Die Ergebnisse der Untersuchungen im Rahmen dieser Arbeit und neue Ergebnisse an laserdeponierten glatten PMMA-Schichten [133] lassen hingegen darauf schließen, dass dieser Vernetzungsprozess auch durch nachträgliche Auslagerung aktiviert werden kann. Dieser Widerspruch lässt sich vermutlich durch eine unterschiedliche Prozessführung im Vorfeld der jeweiligen Messungen erklären: Während die Proben von Scharf direkt nach der Herstellung mit hoher Heizrate weit über ihre Glasübergangstemperatur erhitzt wurden, wurden alle im Rahmen dieser Arbeit untersuchten Messungen direkt nach Herstellung mit den jeweiligen spektroskopischen Methoden untersucht und dazu mit geringer Kühlrate von nur 0,3 K/min er- 
wärmt. Das nach der Laserdeposition defektreiche Polymer und insbesondere die eingeschlossenen Molekülfragmente, die teilweise radikalischen Charakter besitzen, können während des langsamen Heizens zu größeren Strukturen rekombinieren und somit zu starker irreversibler Veränderung des Materials führen. Das schnelle Heizen im Falle der Messungen aus [16] kann hingegen zu einem Abdampfen der reaktiven Molekülfragmente führen, bevor diese neue Bindungen eingehen können. Weiteres Quervernetzen ist damit nicht mehr möglich, die Proben behalten thermoplastischen Charakter und verlieren somit für hohe Temperaturen ihre Dämpfung.

\subsection{Mechanische Dämpfung in Poly(butyl methacrylat)}

Mit dem im Rahmen dieser Arbeit für Tieftemperaturmessungen optimierten PPXR ist es möglich, auch Homologen der PAMAs mit Glasübergangstemperatur unterhalb von Raumtemperatur zu untersuchen. Dazu wurde ein Biegebalken mit Resonanzfrequenz von $2.700 \mathrm{~Hz}$ bei $200 \mathrm{~K}$ mit 10.000 Pulsen Poly(butyl methacrylat) beschichtet und anschließend dessen Dämpfung vermessen. Die entsprechende Schichtdicke ergibt sich über Vergleichsmessungen der Depositionsrate zu etwa 2,4 $\mu \mathrm{m}$.

Abbildung 7.6 zeigt die Entwicklung der Dämpfungsdifferenz dieser Schicht bei sukzessiver Temperaturbehandlung. Die vollen Symbole stellen wieder die Daten der Heiz-, die leeren die der Kühlmessung dar. Nach der Deposition bei $200 \mathrm{~K}$ steigt die Dämpfung an der frischen Probe (schwarze Quadrate) beim Aufheizen zunächst sehr langsam an und zeigt dann bei etwa $245 \mathrm{~K}$ ein schwaches Maximum. Hier führt vermutlich auf dem Biegebalken kondensiertes Material zu zusätzlicher Dämpfung. Da dieses Maximum nur in der Neukurve auftritt, ist davon auszugehen, dass das Material während der ersten Heizmessung wieder abdampft. Dabei kann es sich entweder um Kontaminationen von Restgasen oder aber um leichte Fragmente und Bruchstücke, die seit dem Depositionsprozess auf dem kalten Biegebalken kondensiert sind, handeln.

Im Anschluss an dieses Maximum steigt die Dämpfung bei $275 \mathrm{~K}$ wiederum an und es zeichnet sich ein zweites stärkeres Maximum ab, das den viskosen Fließprozess beschreibt. Für hohe Temperaturen ab $380 \mathrm{~K}$ steigt die Dämpfungsdifferenz schließlich innerhalb weniger Messwerte (von $382,5 \mathrm{~K}$ bis $387 \mathrm{~K}$ ) von $3 \cdot 10^{-5}$ auf $5 \cdot 10^{-4}$ massiv an. In diesem schmalen Temperaturbereich wird das Polymer durch die thermisch induzierte Relaxation sehr schnell 
sehr stark verändert, was zu einem Anstieg der maximalen Dämpfung in der folgenden Kühlmessung auf bis zu $1,6 \cdot 10^{-3}$ bei $360 \mathrm{~K}$ führt. Als Ursache für diese extreme Dämpfungszunahme - selbst in diesem sehr kleinen Temperaturbereich - kommt auch hier, wie im Falle von PEMA und PMMA, nur die thermisch induzierte Relaxation in Frage. Externe, bzw. durch die Messapparatur gegebene Einflüsse sind eindeutig auszuschließen, da die Dämpfungen sowohl der Erstmessung als auch aller weiteren Messungen für niedrige Temperaturen im Rahmen der Messgenauigkeit übereinander liegen. Veränderungen in der Klemmung des Biegebalkens, bzw. ein Lösen des Reeds, welches auch zu einer starken Zunahme der Energiedissipation in der Halterung und somit zu starkem Anwachsen der Dämpfung führte, würde zusätzliche Dämpfung im gesamten Temperaturbereich hervorrufen oder zu unstetigen Sprüngen führen [16]. Beides kann hier nicht beobachtet werden. Weiterhin ist durch die Optimierung der Einspannung am PPXR eine möglichst temperaturunabhängige Art der Klemmung gegeben, s. d. systematische Fehler weitestgehend ausgeschlossen werden können [99].

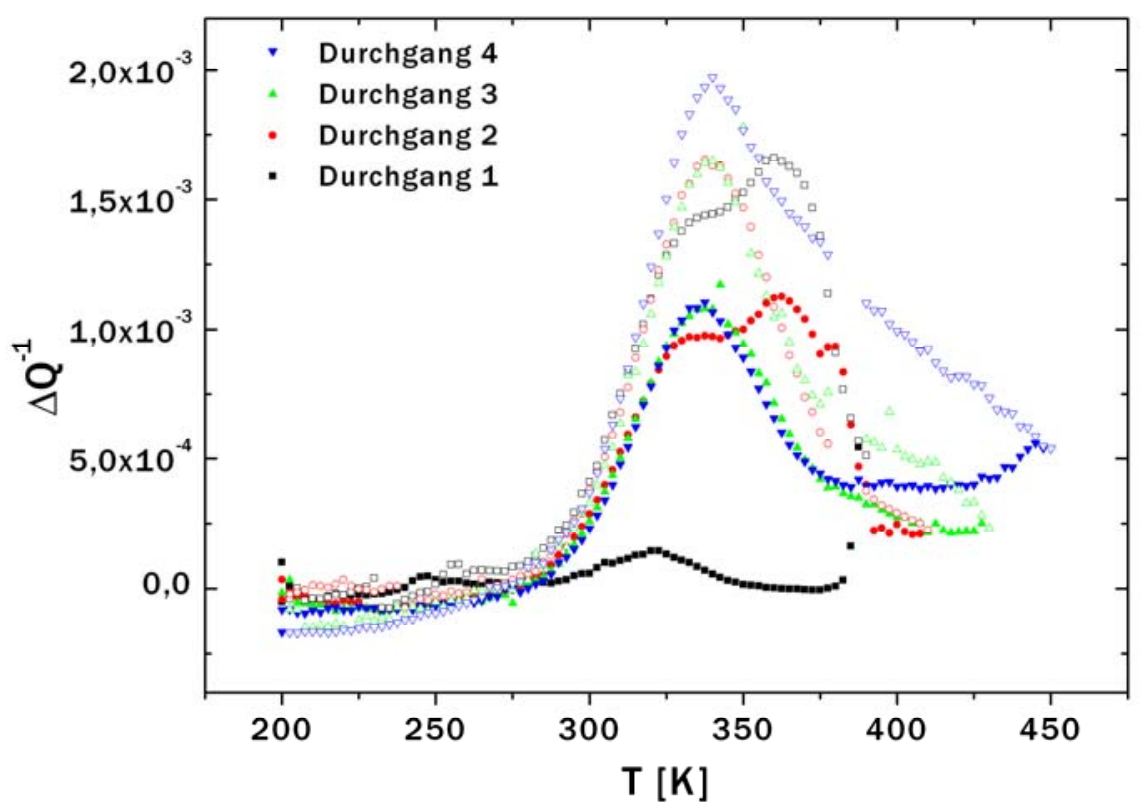

Abbildung 7.6: Temperaturabhängige Dämpfungsdifferenz zwischen dem bei $200 \mathrm{~K}$ mit 10.000 Pulsen PBMA beschichtetem und dem unbeschichteten PPXR bei verschiedenen Auslagerungsdurchgängen. Die vollen Symbole stellen die Daten der Heiz-, die leeren die der Kühlmessung dar.

Sowohl in der ersten Kühlmessung (leere schwarze Quadrate), als auch in der zweiten Heizmessung (rote Punkte) tritt neben dem den Glasübergang beschreibenden Maximum bei etwa $340 \mathrm{~K}$ auch ein starkes zweites Maximum bei etwa $360 \mathrm{~K}$ auf. Da dieses in allen weiteren Folgemessungen nicht mehr messbar ist, scheint hier ein Prozess zugrunde zu liegen, der nach dem zweiten Heizen auf $410 \mathrm{~K}$ vollständig abgeschlossen ist. Ein möglicher Kandidat für 
diesen Prozess ist erneut das Abdampfen von auf dem Biegebalken kondensiertem Material. Durch die enorme Intensität der Dämpfung scheint diese Interpretation aber unwahrscheinlich und es kann vielmehr ein Relaxationsprozess im Polymer angenommen werden. Vermutlich werden während der Herstellung bei tiefen Temperaturen in den PBMA-Schichten Strukturen eingefroren, deren Relaxation über kompliziertere Mechanismen abläuft, als bei den PEMAFilmen. Insbesondere zeigt sich dies hier in der sprunghaften Aktivierung der Relaxation und der Zweistufigkeit. Da ein ähnliches Verhalten in detaillierten Messungen mit dem DPO nicht reproduziert werden konnte (s.u.), sondern stattdessen auch im Falle der PBMA-Filme ein Relaxationsmechanismus gefunden wurde, der dem der PEMA- und PBMA-Filme entspricht, lassen die vorliegenden Ergebnisse eindeutige Schlüsse hier nicht zu, s. d. die tatsächliche Ursache für dieses Verhalten offen bleiben muss.

Neben dem Auftreten des zweiten Maximums zeigt sich erneut die bereits beim PEMA gefundene ungewöhnliche Abhängigkeit der Dämpfung von der thermischen Vorgeschichte (Vgl. Kapitel 6.3.3). Allerdings ist hier die Systematik vertauscht und die Daten der Heizmessungen liegen jeweils unterhalb denen der vorigen Kühlmessungen und zeigen zudem nur wenig Abhängigkeit von der maximalen Auslagerungstemperatur. Als eine mögliche Ursache kommt wieder der bereits für die Interpretation der PEMA-Messungen diskutierte Temperaturgradient in Frage, der zu lokal unterschiedlichen Temperaturen im Polymer und somit zu lokal unterschiedlichen mechanischen Eigenschaften führen kann und damit starken Einfluss auf die Schwingung des Biegebalkens nehmen kann. Als zweite Ursache ist es möglich, dass Polymer, welches mit Erreichen der Glastemperatur zu fließen beginnt, einen Pfad zwischen Biegebalken und Halterung schließt und somit die Energiedissipation an die Halterung verstärkt wird. Dieser Effekt würde durch die thermische Ausdehnung des Polymers gesteigert. Bei niedrigen Temperaturen bricht der Pfad wieder auf und die Dämpfung der folgenden Heizmessung verläuft zunächst mit geringeren Werten. Da an den mikroskopischen Aufnahmen der Polymeroberfläche keine Hinweise auf morphologische Veränderungen der Proben zu finden sind (Vgl. Kapitel 6.5), ist hier jedoch nicht von starkem Materialtransport auszugehen. Dennoch können im kritischen Bereich der Klemmung auch nur geringe Materialverlagerungen zu starken Effekten in der Dämpfung führen. Aufgrund der bei den unterschiedlichen Systemen nicht konsistenten Systematik ist aber auch hier eine eindeutige Zuordnung eines zugrundeliegenden Prozesses nicht möglich.

Systematisch und konsistent mit den Ergebnissen der bisher untersuchten Homologen ist die Zunahme der Dämpfung im Temperaturbereich oberhalb des $\alpha$-Maximums. Hier führt die 
Auslagerung bei Temperaturen oberhalb von $400 \mathrm{~K}$ zum sukzessiven Anstieg in der Dämpfung, s. d. für die letzte Kühlmessung eine stark ausgeprägte Flanke auf der Hochtemperaturseite des $\alpha$-Maximums zu erkennen ist. Die thermisch induzierte Zunahme der Dämpfung bei hohen Temperaturen, die vermutlich auf einen gesteigerten Vernetzungsgrad zurückzuführen ist, lässt sich also wie schon beim PEMA und beim PMMA auch hier nachweisen.

Zur weiteren Analyse wurde auch das PBMA mit unterschiedlichen Schwingungsmoden des DPOs untersucht. Abbildung 7.7 und Abbildung 7.8 zeigen die an einem bei $220 \mathrm{~K}$ mit 1.000 Laserpulsen PBMA beschichteten Oszillator bestimmten Dämpfungsdifferenzen für die AS2Mode bei $5.740 \mathrm{~Hz}$, die AS1-Mode bei $5.020 \mathrm{~Hz}$ und die dritte Biegeschwingungsmode CL3 bei $3.510 \mathrm{~Hz}$ für sechs sukzessive Auslagerungszyklen. Die Schichtdicke kann aus Depositionsratenmessungen an Referenzproben auf $250 \mathrm{~nm}$ abgeschätzt werden.
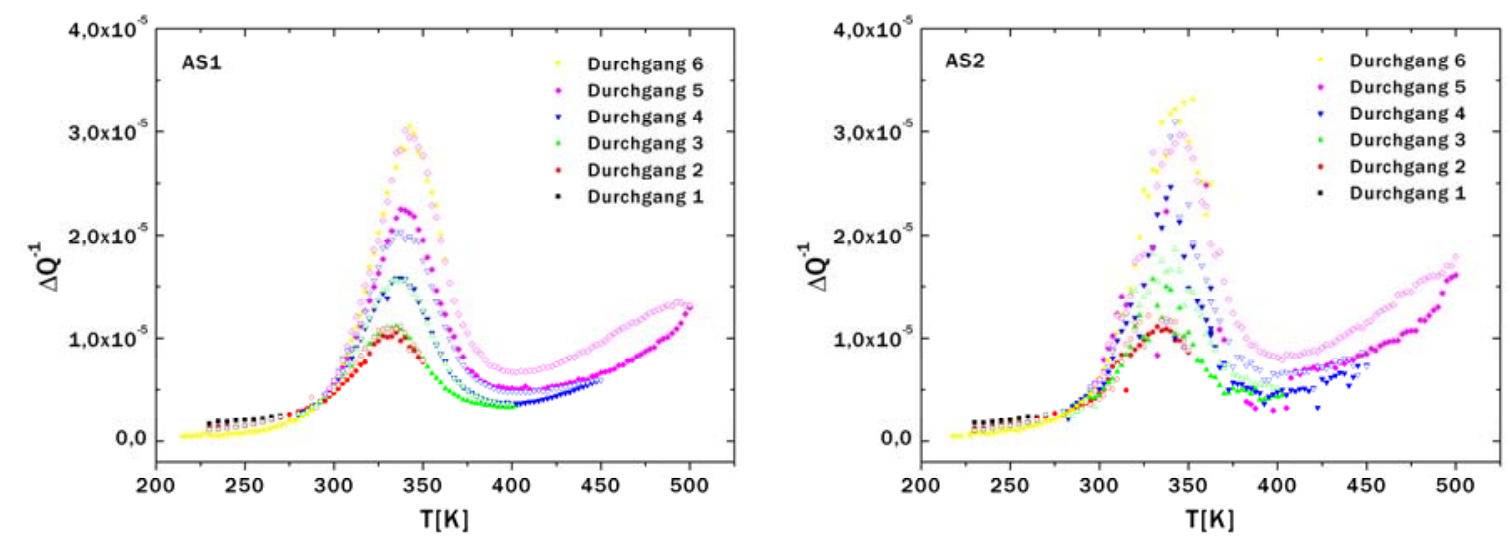

Abbildung 7.7: Abhängigkeit der Dämpfungsdifferenz von der Auslagerung für eine mit 1.000 Pulsen PBMA hergestellten Dünnschicht auf dem DPO, gemessen mit der AS1-Mode bei $5.020 \mathrm{~Hz}$ und der AS2-Mode bei 5.740 Hz. Beide Kurven zeigen die durch die thermisch induzierte Relaxation hervorgerufene Zunahme der Dämpfung mit steigender Auslagerungstemperatur. 


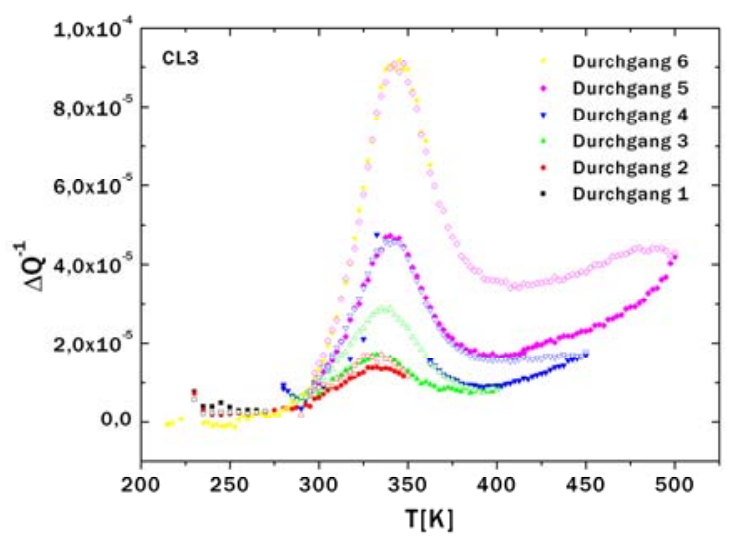

Abbildung 7.8: Abhängigkeit der Dämpfungsdifferenz von der Auslagerung für eine mit 1.000 Pulsen PBMA hergestellten Dünnschicht, gemessen mit CL3-Mode bei $3.510 \mathrm{~Hz}$. Wie auch an den Daten der AS-Moden ist hier der Einfluss der thermisch induzierten Relaxation in einer systematischen Zunahme der Dämpfung zu erkennen. Bedingt durch Anregungsprobleme während des vierten Messdurchgangs konnten hier einige Messwerte nicht aufgenommen werden.

Die mit allen drei Moden bestimmten Dämpfungswerte zeigen den Einfluss der thermisch induzierten Relaxation ohne weitere Artefakte oder Unregelmäßigkeiten. Im ersten Messdurchgang (schwarze Quadrate), der nur bis $270 \mathrm{~K}$, also unterhalb der Glasübergangstemperatur geführt wurde, ist kein signifikanter Anstieg der Dämpfung festzustellen. Stattdessen verläuft die Kühlkurve bei etwas geringeren Werten zurück. Dies ist auf das Abdampfen von Kontaminationen während der ersten Heizmessung zurückzuführen. Nach dem Heizen auf $350 \mathrm{~K}$ im zweiten Heizdurchgang ist die Dämpfung im Maximum der folgenden Kühlmessung nur schwach verstärkt. Erst nach der Auslagerung bei höheren Temperaturen, wie z.B. $400 \mathrm{~K}$ im dritten Durchgang weist die Kühlmessung eine bedeutend stärkere Dämpfung auf als die vorige Heizmessung. Dabei ist die maximale Dämpfung stets abhängig von der maximalen Auslagerungstemperatur, s. d. die jeweiligen Heizmessungen die Daten der vorigen Kühlmessung reproduzieren.

Die Abhängigkeit der maximalen Dämpfung bzw. der Maximumstemperatur von der Auslagerungstemperatur ist in Abbildung 7.9 zusammengefasst. Im linken Teil der Abbildung ist die maximale Dämpfung in einer Arrhenius-Darstellung aufgetragen. Trotz leichter Schwankung in den Messwerten, zeigt sich für alle drei Moden ein einfach thermisch aktiviertes Anwachsen ab einer Schwellentemperatur von etwa $330 \mathrm{~K}$. Diese Temperatur liegt etwa $35 \mathrm{~K}$ unter der für das PEMA bestimmten Schwelle und ca. $35 \mathrm{~K}$ oberhalb der Glasübergangstemperatur des PBMAs. Die mittels Regressionsgeraden bestimmten Aktivierungsenergien von 0,08 eV für die AS-Moden und 0,16 eV für die CL3-Mode entsprechen denen, die auch beim PEMA gefunden wurden. Hier ist also von einem analogen zugrunde liegenden Relaxationsmecha- 
nismus auszugehen, der durch die Mobilität der Kettensegmente limitiert wird, wie bereits in Kapitel 6.6.1 diskutiert wurde.
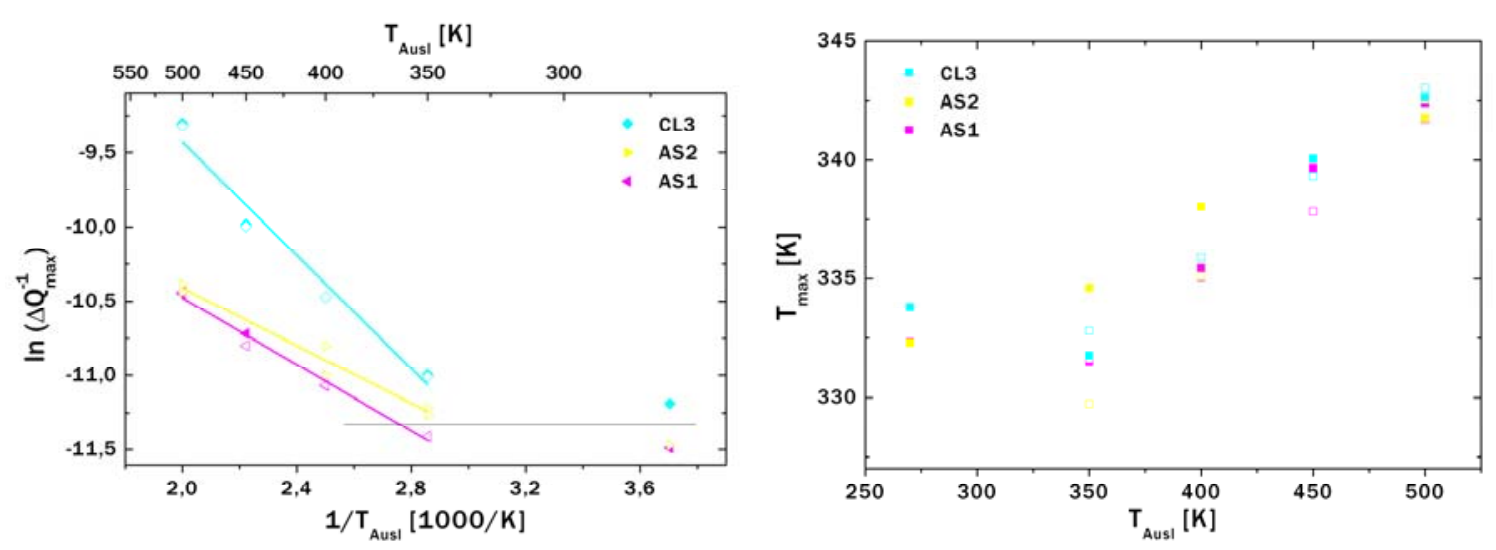

Abbildung 7.9: Abhängigkeit von Höhe und Lage der maximalen Dämpfung von der Auslagerungstemperatur. Links ist der maximale inverse Qualitätsfaktor in einer Arrhenius-Darstellung aufgetragen, rechts ist die Verschiebung der Position des Maximums mit steigender Auslagerungstemperatur gezeigt.

Im rechten Teil von Abbildung 7.9 ist die Position des Dämpfungsmaximums in Abhängigkeit von der Auslagerungstemperatur dargestellt. Hier zeigt sich eine leichte aber signifikante lineare Verschiebung des Maximums zu bis zu $10 \mathrm{~K}$ größeren Werten bei Auslagerungstemperaturen zwischen 270 und $500 \mathrm{~K}$ für alle drei beobachteten Schwingungsmoden. Abermals zeigt sich zwischen den beiden ersten Messwerten bis maximal $350 \mathrm{~K}$ keine signifikante Änderung, s. d. auch hier eine Schwellentemperatur beobachtet werden kann, unter der noch keine Veränderung stattfindet. Es ist zu beachten, dass zwar die Temperatur der maximalen Dämpfung, nicht aber der Onset des Maximums, verschoben wird. Das bedeutet, dass zwar die Glasübergangstemperatur identisch bleibt, hier aber dennoch die Dynamik im Polymer, die knapp oberhalb davon auftritt, zumindest leicht verändert wird. Dämpfungsbeiträge bei höheren Temperaturen beruhen dabei, wie oben bereits für das PEMA diskutiert (Vgl. Kapitel 6.6.1), auf langsameren Prozessen. Die thermisch induzierte Relaxation führt also auch im Falle des PBMAs dazu, dass größere Kettensegmente an der Schwingung beteiligt werden und somit zur Energiedissipation beitragen können.

Zur Vervollständigung der Vergleichsmöglichkeiten der Frequenz- und Modenabhängigkeit sind in Abbildung 7.10 die Dämpfungsdifferenzen von PBMA-Proben mit vergleichbarem Relaxationszustand nach Auslagerung bei $450 \mathrm{~K}$ aufgetragen. Zusätzlich dargestellt ist die letzte Heizkurve der PPXR-Messung (volle blaue Dreiecke), hier wurde die Probe zuvor nur bei $430 \mathrm{~K}$ ausgelagert. Im linken Teil der Abbildung sind die Absolutwerte der Dämpfungsdifferenz in logarithmischer Skala dargestellt. Alle Kurven zeigen hier klar das zum $\alpha$-Prozess 
gehörige Maximum. Die mit dem DPO gewonnenen Daten weisen dabei sehr gering Dämpfung auf, wobei die AS-Moden aufgrund ihrer hohen Güte wiederum minimale Dämpfung liefern. Die mit dem PPXR gemessene Dämpfungsdifferenz liegt um fast zwei Größenordnungen höher, was neben methodischen Unterschieden aufgrund der unterschiedlichen Schwingungsgüte auch durch die um den Faktor 10 erhöhte Schichtdicke verursacht wird. Wie bereits bei den vergleichenden Auftragungen in Kapitel 6.6 ist auch hier die PPXRTemperaturskala (oben) im Vergleich zur DPO-Temperaturskala (unten) verschoben, da die Ofengeometrie für diese Messungen weiter optimiert wurde, reicht in diesem Fall eine Verschiebung von $5 \mathrm{~K}$ aus, um die Maxima zur Deckung zu bringen. Da die Ofenparameter für diese Messungen sehr ähnlich waren, kann die Verschiebung der Temperaturskala hier unter Umständen auch physikalische Ursachen haben und auf der unterschiedlichen Messfrequenz beruhen. Da jedoch weitere Vergleichsmessungen fehlen und der apparative Fehler der Temperaturbestimmung in einem ähnlichen Bereich liegt, wird die Temperaturverschiebung wie auch bei den Untersuchungen der weiteren Homologen als apparativer Offset gedeutet.
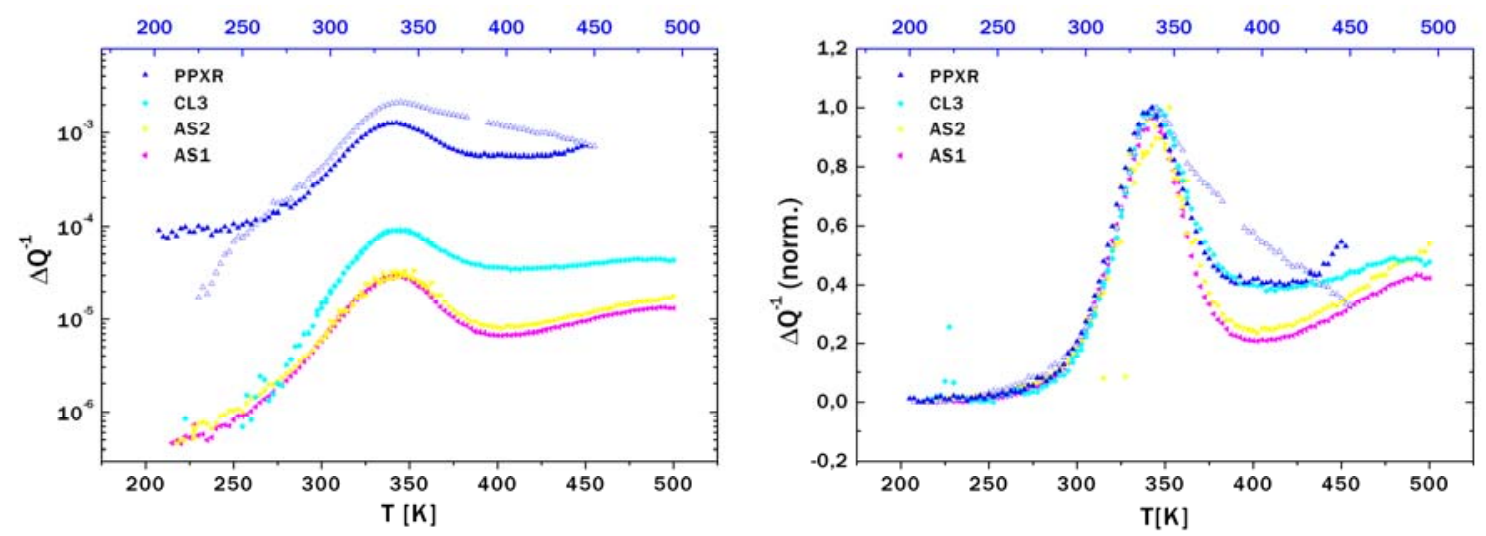

Abbildung 7.10: Vergleich der Dämpfungskurven von PBMA-Schichten für die unterschiedlichen Schwingungsmoden des DPOs sowie des PPXRs, links in logarithmierter, rechts in normierter Auftragung.

In der normierten Darstellung der Dämpfungswerte im rechten Teil von Abbildung 7.10 lassen sich die qualitativen Kurvenformen sehr gut vergleichen. Es zeigt sich hier für alle Messungen ein ähnlicher Verlauf mit geringer Dämpfung bei tiefen Temperaturen, dem klaren Maximum bei $340 \mathrm{~K}$ und auch bei hohen Temperaturen nicht vollständig verschwindender Dämpfung. Damit kann auch für das PBMA, wie bereits beim PEMA und PMMA kein signifikanter Einfluss der Art des verwendeten Oszillators bzw. der Art der Schwingung auf die Dynamik im Polymer gefunden werden. 
Aus dem Anstieg zum Maximum lässt sich modenunabhängig die Glasübergangstemperatur $\mathrm{zu} T_{\mathrm{g}}=298 \mathrm{~K}$ bestimmen. Auch auf der Hochtemperaturflanke ist für alle Kurven ein ähnlicher Verlauf zu erkennen. Lediglich die relative Stärke der Dämpfung im mittleren Temperaturbereich variiert, wobei die Biegeschwingungsmoden hier einen höheren Dämpfungsanteil aufweisen. Diese Unterschiede sind aber vermutlich mehr auf die unterschiedliche Qualität der Messung bzw. Schwingungsgüte des verwendeten Oszillators als auf systematische Unterschiede in der Art der Schwingung zurückzuführen. Auch die Abweichungen im Hochtemperaturbereich der PPXR-Messung an der bei $450 \mathrm{~K}$ ausgelagerten Probe stellen dabei keine Ausnahme dar.

Der detaillierte Vergleich der temperaturabhängigen mechanischen Eigenschaften der untersuchten laserdeponierten Poly(alkyl methacrylat)e liefert keine Hinweise auf fundamental unterschiedliche dynamische Prozesse zwischen den jeweiligen Homologen. Vielmehr zeigen alle gewonnenen Verlustspektren - unter Vernachlässigung der nicht eindeutig zu identifizierenden Besonderheiten in einzelnen Messungen - im untersuchten Frequenz- und Temperaturbereich ein starkes Dämpfungsmaximum, dessen dynamische Ursache als $\alpha$-Relaxation, also als viskoser Fließprozess identifiziert werden kann. Während die absolute Temperatur, bei der der Fließprozess einsetzt, für die verschiedenen Homologen aufgrund der internen Plastifikation stark von den Details der Alkyl-Seitengruppen abhängt, und somit Glasübergangstemperaturen zwischen $298 \mathrm{~K}$ (PBMA), $330 \mathrm{~K}$ (PEMA) und $375 \mathrm{~K}$ (PMMA) gefunden werden, ist die temperaturabhängige Charakteristik des Fließprozesses weitestgehend unabhängig von der Seitengruppe, was sich anhand der für alle Materialien ähnlichen Verlustspektren manifestiert.

Diese Verlustspektren sind auch unabhängig von der verwendeten Messmethode, insbesondere von der Art der Schwingung. Im Falle von PMMA und PBMA zeigt sich lediglich eine schwache Zunahme der Temperatur der maximalen Dämpfung mit steigender Messfrequenz. Auch für das PEMA kann eine solche Abhängigkeit nicht ausgeschlossen werden. Signifikante Unterschiede zwischen den mit Biege- bzw. mit Torsionsschwingungen aufgenommenen Spektren können jedoch nicht festgestellt werden.

Ebenfalls unabhängig von Material und Untersuchungsmethode kann die thermisch induzierte Relaxation als universelle Eigenschaft laserdeponierter PAMAs gefunden werden. Sie wird 
als thermisch aktivierter Prozess mit typischen Aktivierungsenergien zwischen $0,1 \mathrm{eV}$ und $0,3 \mathrm{eV}$ identifiziert. Für alle Homologen finden sich dabei Schwellentemperaturen, oberhalb derer die Relaxation signifikant einsetzt und zum Anstieg der gemessen Dämpfung führt. Diese liegen jeweils etwa $30 \mathrm{~K}$ oberhalb der jeweiligen Glastemperatur. Da also die ausreichende Beweglichkeit der Kettensegmente als notwendige Bedingung für das Einsetzen der Relaxation ebenfalls als Universalität für die laserdeponierten PAMA-Filme gefunden wird, lassen sich die am PEMA gefunden Erkenntnisse bzgl. des zugrunde liegenden Prozesses auf die weiteren Homologen übertragen. 



\section{Zusammenfassung}

$\mathrm{Zu}$ Beginn dieser Arbeit war der grundlegende Mechanismus der gepulsten Laserdeposition von Poly(methyl methacrylat) weitestgehend bekannt und die Eigenschaften der resultierenden PMMA-Filme konnten mit diesem gut beschrieben werden. Das Ziel dieser Arbeit war es, zu prüfen, in wie weit sich diese Einsichten auf die weiteren Vertreter der Poly(alkyl methacrylat)e verallgemeinern lassen und so ein umfassendes, möglichst universelles Verständnis sowohl der Ablations- und Depositionsmechanismen als auch der Filmeigenschaften für alle Homologen aufzubauen. Dabei standen insbesondere die durch die interne Dynamik der amorphen polymeren Systeme bestimmten frequenz- und temperaturabhängigen Eigenschaften im Fokus dieser Arbeit. Von zentraler Bedeutung war dabei stets die Frage nach Universalität, sowohl in Hinblick auf die Eigenschaften der einzelnen Alkylmethacrylate, als auch auf die zugrundeliegenden Mechanismen.

Durch die gezielte Analyse jener Filmeigenschaften, die direkte Rückschlüsse auf den Depositionsmechanismus erlauben, konnte beispielhaft für das laserdeponierte Poly(ethyl methacrylat) ein dem PMMA ähnlicher Mechanismus gefunden werden. Zusammen mit den Ergebnissen am Poly(butyl methacrylat) lässt sich dieser auf die ersten vier Homologen erweitern und somit ein universelles Bild der PAMA-Filme aufbauen:

Laserdeponierte Poly(alkyl methacrylat)-Filme zeichnen sich durch zwei Komponenten aus. Der glatte Filmanteil aus stark vernetztem, unlöslichem Material zeigt Oberflächenrauigkeiten weit unter einem Nanometer und resultiert aus der Reaktion von kleineren Fragmenten, die durch photothermische und photochemische Prozesse während der Laserdeposition aus dem Target gelöst werden und dann teilweise im Flug bzw. auf dem Substrat rekombinieren und repolymerisieren. Die zweite Komponente besteht aus vielen bis zu mehreren Mikrometer großen Tröpfchen (Droplets), die in flüssiger Form vom Target zum Substrat transferiert werden und hier erstarren. Sie machen mit etwa vier Fünfteln der Gesamtmasse den größten Anteil der laserdeponierter PAMA-Filme aus. Ihre Entstehung kann durch eine Kombination aus 
photochemischer Inkubation und photothermischem Abtrag erklärt werden. Die Inkubation führt im Targetmaterial zu einer Verkürzung der Kettenlängen verbunden mit der Bildung von zusätzlichen Doppelbindungen. Diese dienen dann als Chromophore für die UV-Strahlung, s. d. die Absorption im Target sukzessive erhöht wird. Die zum photothermischen Abtrag ganzer Tröpfchen benötigte kritische Absorption ist für die PAMAs erreicht, wenn die molekulare Masse im Target durch Inkubation auf Werte zwischen 8 und $9 \mathrm{~kg} / \mathrm{mol}$ abgesenkt wurde.

Die Reihe der Poly(alkyl methacrylat)e bietet sich als Modellsystem zur Untersuchung der Dynamik in Polymeren an, weil hier durch sukzessive Verlängerung der Seitengruppen drastische Veränderungen der internen Relaxationsprozesse hervorgerufen werden, während die sonstigen physikalischen und chemischen Eigenschaften nur schwach modifiziert werden. Im Rahmen dieser Arbeit wurden mit der mechanischen und der dielektrischen Spektroskopie zwei komplementäre Methoden verwendet, um die hier zugrundeliegenden dynamischen Prozesse zu verstehen. Dazu wurden vorhandene experimentelle Aufbauten erweitert, optimiert und teilweise neu entwickelt. Im Rahmen der Entwicklung und dem Aufbau der verschiedenen Apparaturen wurden umfassende Charakterisierungen vorgenommen, vergleichende Simulationen mit Finite-Elemente-Modellierungen durchgeführt und Steuer- sowie Auswertungsprogramme entworfen. Als experimenteller Höhepunkt steht mit dem Bau der AllMode-DPO-Anlage im Rahmen dieser Arbeit neuerdings eine hochsensible Methode zur Verfügung, mit der im Temperaturbereich zwischen 200 und $700 \mathrm{~K}$ in-situ frequenzabhängige, mechanische Eigenschaften laserdeponierter Filme bestimmt werden können.

Als absoluter Bezugspunkt diente bei der Untersuchung der dynamischen Eigenschaften wieder das Beispielsystem PEMA, welches umfassend und quantitativ untersucht wurde. Zur Einordnung der weiteren Homologen in das dabei gefundene Bild ist deren quantitative Charakterisierung dann nicht mehr zwingend notwendig.

Während die mechanischen Eigenschaften laserdeponierter Filme direkt nach der Herstellung nicht temperaturstabil sind und bei thermischer Auslagerung zunächst irreversibel verändert werden, findet man bei relaxierten Proben, die bereits weit über ihre Glasübergangstemperatur erhitzt wurden, ein dem bulk-Material ähnliches Verhalten mit einem einfachen Glasübergang. Im Vergleich zum bulk-PEMA liegen die mechanischen Moduln dieser Proben jedoch bei stark erhöhten Absolutwerten: Der mit dem Doppelpaddeloszillator bei einer Messfrequenz von $5.100 \mathrm{~Hz}$ bestimmte Speichermodul beträgt bei Raumtemperatur $G^{\prime}(300 \mathrm{~K})=9 \mathrm{GPa}$ und fällt nach dem Glasübergang auf $G^{\prime}(425 \mathrm{~K})=6 \mathrm{GPa}$ ab. Der zugehö- 
rige Verlustmodul zeigt einen Anstieg von etwa $G^{\prime \prime}(300 \mathrm{~K})=0,1 \quad \mathrm{GPa}$ auf $G^{\prime \prime}(385 \mathrm{~K})=0,4 \mathrm{GPa}$. Die entsprechenden Werte des mit dem Plasma Plume eXcited Reed bestimmten Elastizitätsmoduls liegen mit $E^{\prime}{ }_{\max }=9 \mathrm{GPa}$ und $E^{\prime \prime}{ }_{\max }=1,1 \mathrm{GPa}$ in der gleichen Größenordnung.

Im Hochtemperaturbereich weit oberhalb des Glasübergangs weisen sowohl die Speicher- als auch die Verlustmoduln ausgelagerter PEMA-Filme auch bei hohen Temperaturen endliche Werte auf. Im Falle der Speichermoduln lässt dies statt des bulk-artigen thermoplastischen Verhaltens eher auf einen duroplastischen Charakter schließen. Dabei ist der Einfluss der sich bereits durch den Depositionsprozess im Film befindenden vernetzten Komponente vernachlässigbar. Diese und weitere Abweichungen vom bulk-Verhalten resultieren vielmehr aus einer thermisch induzierten Relaxation, die während thermischer Auslagerung sowohl zur Zunahme der Verlust-, als auch der Speichermoduln führt und somit insbesondere nicht mit typischen Alterungsprozessen zu verwechseln ist. Diese Relaxationsart konnte für die PAMAs als universeller Prozess gefunden werden. Aufgrund der beobachteten Aktivierungsenergie zwischen $0,1 \mathrm{eV}$ und $0,3 \mathrm{eV}$ und der Schwellentemperaturen, unter denen keine Relaxation auftritt - diese liegen für alle Homologen etwa $30 \mathrm{~K}$ oberhalb der Glasübergangstemperatur kann die Diffusion von Kettensegmenten hier als die Relaxation limitierender Prozess angegeben werden. Detailliert führen dann unterschiedliche Prozesse, deren eindeutige Identifikation nicht möglich ist, zur Erhöhung der Moduln. Wahrscheinlichster Kandidat für die $\mathrm{Zu}$ nahme des Verlustmoduls ist die durch Reorientierung von Ketten bzw. Kettensegmenten bedingte Kompaktierung des Materials. Hier können thermisch aktiviert Verschlaufungen gelöst werden, die noch durch den Depositionsprozess im Material vorhanden sind. Durch dieses Ausheilen können größere Kettensegmente kollektiv an der Verformung teilnehmen und durch innere Reibung zu Energiedissipation im gesamten Temperaturbereich beitragen. Die Zunahme der Speichermoduln kann nur über eine Quervernetzung von Makromolekülen erklärt werden. Da die thermische Energie und die gefundenen Aktivierungsenergien aber nicht ausreichen, um aktiv Bindungen zu brechen, muss dieser Vernetzungsprozess über instabile Moleküle und Fragmente, die Radikale bilden können ,ablaufen. Diese sind durch die UV-Strahlung im Film vorhanden und bei Raumtemperatur in ihrer Beweglichkeit kinetisch durch die umliegenden Ketten gehemmt, s. d. auch dieser Prozess indirekt über die Diffusion der Kettensegmente thermisch aktiviert werden muss.

Auch die mittels der temperaturabhängigen dielektrischen Spektroskopie aufgenommenen Verlustspektren zeigen in einem weiten Temperaturbereich bulk-ähnliches Verhalten und die 
gemessenen Verlustmaxima können wie erwartet eindeutig der $\beta$-Relaxation zugeordnet werden. Da diese $\beta$-Relaxation mit Kippbewegungen in der Hauptkette korreliert ist, zeigt sich hier indirekt auch im dielektrischen Verlust der Einfluss des Fließprozesses. Bei hohen Temperaturen setzen irreversible Veränderungen ein, die zu teilweise unverstandenem Verhalten führen.

Die für PEMA-Filme bestimmten Werte der Gleichstromleitfähigkeit liegen um viele Größenordnungen oberhalb denen von bulk-Proben. Dies führt zu der Annahme, dass die oben erwähnten im Film eingeschlossen Moleküle und Fragmente hier als zusätzliche Ladungsträger auftreten können. Diese Interpretation wird gestärkt durch die gefundenen Aktivierungsenergien, die wieder auf die Diffusion von Kettensegmenten hindeuten.

Insgesamt konnte im Verlauf dieser Arbeit ein weitgehend homogenes und universelles Bild sowohl für die Depositionsmechanismen als auch für die Eigenschaften laserdeponierter Filme aus Poly(alkyl methacrylat) aufgebaut werden. Während im Depositionsmechanismus keine signifikanten Unterschiede zu erkennen sind, unterscheiden sich die untersuchten temperatur- und frequenzabhängigen Eigenschaften innerhalb der Homologen meist nur durch die über die interne Plastifikation bedingten Abweichungen in der Glastemperatur und weisen in weiten Bereichen bulk-ähnliches Verhalten auf. Gleichzeitig sind die Eigenschaften der Filme geprägt durch markante temperaturabhängige Prozesse, wie die thermisch induzierte Relaxation, die eindeutig auf Veränderungen des Polymers während des Depositionsprozesses zurückzuführen sind und somit klar PLD-spezifisch sind. 


\section{Literaturverzeichnis}

[1] W. J. Thieman, M. A. Palladino, and N. W. Hopf, Biotechnologie (Pearson Studium, München [u. a., 2007).

[2] G. W. Ehrenstein, Dissertation, Polymer-Werkstoffe : Struktur - Eigenschaften - Anwendung ; mit 22 Tabellen (Hanser, München; Wien, 1999).

[3] I. M. Campbell, Introduction to synthetic polymers (Oxford University Press, Oxford [u.a.], 1996).

[4] D. B. Chrisey et al., Laser deposition of polymer and biomaterial films, Chemical Reviews 103, 553 (2003).

[5] A. J. Heeger, Nobel Lecture: Semiconducting and metallic polymers: The fourth generation of polymeric materials, Reviews of Modern Physics 73, 681 (2001).

[6] A. G. MacDiarmid, Nobel Lecture: "Synthetic metals": A novel role for organic polymers, Reviews of Modern Physics 73, 701 (2001).

[7] H. Shirakawa, Nobel Lecture: The discovery of polyacetylene film: The dawning of an era of conducting polymers, Reviews of Modern Physics 73, 713 (2001).

[8] K. Norrman, A. Ghanbari-Siahkali, and N. B. Larsen, Studies of spin-coated polymer films, Annual Reports Section "C" (Physical Chemistry) 101, 174 (2005).

[9] H. Biederman, and Y. Osada, Plasma polymerization processes (Elsevier ; Distributors for the U.S. and Canada, Elsevier Science Pub. Co., Amsterdam; New York; New York, N.Y., U.S.A., 1992).

[10] S. G. Hansen, and T. E. Robitaille, Formation of Polymer-Films by Pulsed Laser Evaporation, Applied Physics Letters 52, 81 (1988).

[11] T. Lippert, R. L. Webb, S. C. Langford, and J. T. Dickinson, Dopant induced ablation of poly (methyl methacrylate) at $308 \mathrm{~nm}$, Journal of Applied Physics 85, 1838 (1999).

[12] S. Küper, S. Modaressi, and M. Stuke, Photofragmentation Pathways of a Pmma Model-Compound under Uv Excimer Laser Ablation Conditions, Journal of Physical Chemistry 94, 7514 (1990).

[13] S. Küper, and M. Stuke, Uv-Excimer-Laser Ablation of Polymethylmethacrylate at 248 $\mathrm{Nm}$ - Characterization of Incubation Sites with Fourier-Transform Ir-Spectroscopy and UvSpectroscopy, Applied Physics a-Materials Science \& Processing 49, 211 (1989).

[14] R. Srinivasan, Ablation of Polymethyl Methacrylate Films by Pulsed (Ns) Ultraviolet and Infrared (9.17-Mu-M) Lasers - a Comparative-Study by Ultrafast Imaging, Journal of Applied Physics 73, 2743 (1993). 
[15] E. Süske, Dissertation, Charakteristische Eigenschaften laserdeponierter Poly(methylmethacrylat)-Filme (Georg-August Universität, Göttingen, 2005).

[16] T. Scharf, Dissertation, Depositionsmechanismen, Struktur und mechanische Eigenschaften laserdeponierter Poly(methyl methacrylat)-Filme (Georg-August Universität, Göttingen, 2006).

[17] E. Süske et al., Variation of the mechanical properties of pulsed laser deposited PMMA films during annealing, Applied Physics a-Materials Science \& Processing 79, 1295 (2004).

[18] E. Süske et al., Tuning of cross-linking and mechanical properties of laser-deposited poly (methyl methacrylate) films, Journal of Applied Physics 97 (2005).

[19] P. W. Anderson, Through the Glass Lightly, Science 267, 1615 (1995).

[20] S. R. Elliott, Physics of amorphous materials (Longman Scientific \& Technical, Harlow, 1989).

[21] C. Janot, Quasicrystals : a primer (Clarendon Press, Oxford, 1994).

[22] J. Zarzycki, Glasses and the vitreous state (Cambridge University Press, Cambridge; New York, 1991).

[23] W. Kauzmann, The Nature of the Glassy State and the Behavior of Liquids at Low Temperatures, Chemical Reviews 43, 219 (1948).

[24] K. Binder, From orientational glasses to structural glasses: What computer simulations have contributed to understand experiments, Journal of Non-Crystalline Solids 307, 1 (2002).

[25] R. C. Plumb, Antique Windowpanes and the Flow of Supercooled Liquids, Journal of Chemical Education 66, 994 (1989).

[26] L. C. E. Struik, Physical aging in amorphous polymers and other materials (Elsevier Scientific Pub. Co. ; Distributors for the U.S. and Canada, Elsevier North-Holland, Amsterdam; New York; New York, 1978).

[27] C. A. Angell, Formation of Glasses from Liquids and Biopolymers, Science 267, 1924 (1995).

[28] D. J. Plazek, and K. L. Ngai, Correlation of Polymer Segmental Chain Dynamics with Temperature-Dependent Time-Scale Shifts, Macromolecules 24, 1222 (1991).

[29] J. L. G. Ribelles et al., Structural Relaxation of Glass-Forming Polymers Based on an Equation for Configurational Entropy .2. Structural Relaxation in Polymethacrylates, Macromolecules 28, 5878 (1995).

[30] M. Wind, R. Graf, A. Heuer, and H. W. Spiess, Structural Relaxation of Polymers at the Glass Transition: Conformational Memory in Poly(n-alkylmethacrylates), Physical Review Letters 91, 155702 (2003).

[31] R. Böhmer, and C. A. Angell, Elastic and Viscoelastic Properties of Amorphous Selenium and Identification of the Phase-Transition between Ring and Chain Structures, Physical Review B 48, 5857 (1993).

[32] T. Odagaki, Glass-Transition Singularities, Physical Review Letters 75, 3701 (1995).

[33] P. Lunkenheimer, U. Schneider, R. Brand, and A. Loidl, Glassy dynamics, Contemporary Physics 41, 15 (2000). 
[34] S. Hensel-Bielowka, and M. Paluch, Origin of the high-frequency contributions to the dielectric loss in supercooled liquids, Physical Review Letters 89 (2002).

[35] H. Tanaka, Origin of the excess wing and slow beta relaxation of glass formers: A unified picture of local orientational fluctuations, Physical Review E 69 (2004).

[36] K. L. Ngai, Comment on "Origin of the excess wing and slow beta relaxation of glass formers: A unified picture of local orientational fluctuations", Physical Review E 70 (2004).

[37] H. Tanaka, Reply to "Comment on 'Origin of the excess wing and slow beta relaxation of glass formers: A unified picture of local orientational fluctuations' ", Physical Review E 70 (2004).

[38] K. L. Ngai, Johari-Goldstein relaxation as the origin of the excess wing observed in metallic glasses, Journal of Non-Crystalline Solids 352, 404 (2006).

[39] N. G. McCrum, B. E. Read, and G. Williams, Anelastic and dielectric effects in polymeric solids (John Wiley, London; New York [etc., 1967).

[40] K. Schmidtrohr et al., Molecular Nature of the Beta-Relaxation in Poly(Methyl Methacrylate) Investigated by Multidimensional NMR, Macromolecules 27, 4733 (1994).

[41] G. P. Johari, and M. Goldstein, Viscous Liquids and Glass Transition .2. Secondary Relaxations in Glasses of Rigid Molecules, Journal of Chemical Physics 53, 2372 (1970).

[42] A. Kudlik et al., Slow secondary relaxation in simple glass formers, Journal of NonCrystalline Solids 235, 406 (1998).

[43] A. Kudlik et al., The dielectric response of simple organic glass formers, Journal of Molecular Structure 479, 201 (1999).

[44] P. Rösner, K. Samwer, and P. Lunkenheimer, Indications for an "excess wing" in metallic glasses from the mechanical loss modulus in $\mathrm{Zr}_{65} \mathrm{Al}_{7.5} \mathrm{Cu}_{27.5}$, Europhysics Letters 68, 226 (2004).

[45] U. Schneider, R. Brand, P. Lunkenheimer, and A. Loidl, Excess wing in the dielectric loss of glass formers: A Johari-Goldstein beta relaxation?, Physical Review Letters 84, 5560 (2000).

[46] P. Lunkenheimer, and A. Loidl, Dielectric spectroscopy of glass-forming materials: alpha-relaxation and excess wing, Chemical Physics 284, 205 (2002).

[47] J. C. Dyre, A model for the generic alpha relaxation of viscous liquids, Europhysics Letters 71, 646 (2005).

[48] G. Adam, and J. H. Gibbs, On Temperature Dependence of Cooperative Relaxation Properties in Glass-Forming Liquids, Journal of Chemical Physics 43, 139 (1965).

[49] G. P. Johari, Glass-Transition and Secondary Relaxations in Molecular Liquids and Crystals, Annals of the New York Academy of Sciences 279, 117 (1976).

[50] A. P. Sokolov et al., The Dynamics of Strong and Fragile Glass Formers - Vibrational and Relaxation Contributions, Journal of Non-Crystalline Solids 172, 138 (1994).

[51] A. I. Chumakov et al., Collective nature of the boson peak and universal transboson dynamics of glasses, Physical Review Letters 92 (2004).

[52] C. A. Angell, Boson peaks and floppy modes: some relations between constraint and excitation phenomenology, and interpretation, of glasses and the glass transition, Journal of Physics-Condensed Matter 16, S5153 (2004). 
[53] U. Buchenau, and H. R. Schober, An atomic mechanism for the boson peak in metallic glasses, Philosophical Magazine 88, 3885 (2008).

[54] A. Kursumovic, and B. Cantor, Anelastic crossover and creep recovery spectra in $\mathrm{Fe}_{40} \mathrm{Ni}_{40} \mathrm{~B}_{20}$ metallic glass, Scripta Materialia 34, 1655 (1996).

[55] R. Kohlrausch, Theorie des elektrischen Rückstandes in der Leidener Flasche, Annalen der Physik und Chemie 167, 56 (1854).

[56] G. Williams, and D. C. Watts, Non-Symmetrical Dielectric Relaxation Behaviour Arising from a Simple Empirical Decay Function, Transactions of the Faraday Society 66, 80 (1970).

[57] F. Alvarez, A. Alegria, and J. Colmenero, Relationship between the Time-Domain Kohlrausch-Williams-Watts and Frequency-Domain Havriliak-Negami Relaxation Functions, Physical Review B 44, 7306 (1991).

[58] S. Havriliak, and S. Negami, A Complex Plane Representation of Dielectric and Mechanical Relaxation Processes in Some Polymers, Polymer 8, 161 (1967).

[59] F. Alvarez, A. Alegria, and J. Colmenero, Interconnection between Frequency-Domain Havriliak-Negami and Time-Domain Kohlrausch-Williams-Watts Relaxation Functions, Physical Review B 47, 125 (1993).

[60] S. S. N. Murthy, Secondary Relaxations and the Structure of Glass, Journal of the Chemical Society-Faraday Transactions Ii 85, 581 (1989).

[61] F. H. Stillinger, A Topographic View of Supercooled Liquids and Glass-Formation, Science 267, 1935 (1995).

[62] P. G. Debenedetti, and F. H. Stillinger, Supercooled liquids and the glass transition, Nature 410, 259 (2001).

[63] W. Götze, and L. Sjögren, Relaxation Processes in Supercooled Liquids, Reports on Progress in Physics 55, 241 (1992).

[64] U. Bengtzelius, W. Götze, and A. Sjolander, Dynamics of Supercooled Liquids and the Glass-Transition, Journal of Physics C-Solid State Physics 17, 5915 (1984).

[65] F. P. Reding, R. D. Whitman, and J. A. Faucher, Glass Transitions in Ethylene Copolymers and Vinyl Homopolymers and Copolymers, Journal of Polymer Science 57, 483 (1962).

[66] B. Wunderlich, Motion in Polyethylene .3. Amorphous Polymer, Journal of Chemical Physics 37, 2429 (1962).

[67] R. F. Boyer, The relation of transition temperatures to chemical structure in high polymers, Rubber Chem. Technol. 36, 1303 (1963).

[68] P. G. DeGennes, Reptation of a Polymer Chain in Presence of Fixed Obstacles, Journal of Chemical Physics 55, 572 (1971).

[69] P. Grünberg, 14. Viskoelastische Eigenschaften in Physik der Polymere,Vorlesungsmanuskripte des 22. IFF-Ferienkurses im Forschungszentrum Jülich, edited by R. Hölzle1991), p. 14.20.14. Viskoelastische Eigenschaften

[70] L. H. Sperling, Introduction to physical polymer science (Wiley, New York, NY [u.a.], 1992). 
[71] S. C. Kuebler et al., 2D exchange NMR investigation of the alpha-relaxation in poly(ethyl methacrylate) as compared to poly(methyl methacrylate), Macromolecules 30, 6597 (1997).

[72] P. W. Smith, Stabilized Single-Frequency Output from a Long Laser Cavity, Ieee Journal of Quantum Electronics Qe 1, 343 (1965).

[73] D. Dijkkamp et al., Preparation of $\mathrm{Y}$-Ba-Cu Oxide Superconductor Thin-Films Using Pulsed Laser Evaporation from High-Tc Bulk Material, Applied Physics Letters 51, 619 (1987).

[74] S. Fähler, and H. U. Krebs, Calculations and experiments of material removal and kinetic energy during pulsed laser ablation of metals, Applied Surface Science 96-8, 61 (1996).

[75] D. B. Chrisey, and G. K. Hubler, Pulsed laser deposition of thin films (J. Wiley, New York, 1994).

[76] A. Piqué et al., Laser processing of polymer thin films for chemical sensor applications, Surface \& Coatings Technology 163, 293 (2003).

[77] J. Brandenburg et al., Pulsed laser deposition of metals: consequences of the energy distribution within the laser spot on film growth, Applied Physics A-Materials Science \& Processing 79, 1005 (2004).

[78] R. Srinivasan, K. G. Casey, B. Braren, and M. Yeh, The Significance of a Fluence Threshold for Ultraviolet-Laser Ablation and Etching of Polymers, Journal of Applied Physics 67, 1604 (1990).

[79] B. D. Cullity, and S. R. Stock, Elements of X-ray diffraction (Pearson/Prentice Hall, Upper Saddle River, 2001).

[80] D. L. Windt, IMD - Software for modeling the optical properties of multilayer films, Computers in Physics 12, 360 (1998).

[81] L. G. Parratt, Surface Studies of Solids by Total Reflection of X-Rays, Physical Review 95, 359 (1954).

[82] H. Günzler, and H. M. Heise, IR-Spektroskopie. Eine Einführung Weinheim [u.a.]: $\mathrm{VCH}, 1996)$.

[83] J. L. Snoek, Effect of small quantities of carbon and nitrogen on the elastic and plastic properties of iron, Physica 8, 711 (1941).

[84] T. Lindveit, P. Peguin, and P. Gobin, Method for Automatic Measurement of Internal Friction by Torsion Pendulum, Journal of Scientific Instruments 41, 564 (1964).

[85] M. Koiwa, and R. R. Hasiguti, A Theory of Internal Friction Peak Due to Thermal Unpinning of Dislocations and Its Application to P1 Peak in Copper, Acta Metallurgica 13, $1219(1965)$

[86] G. S. Baker, and S. H. Carpente, Simultaneous Deformation and Internal Friction Measurements, Review of Scientific Instruments 36, 29 (1965).

[87] W. Benoit, G. Gremaud, and B. Quenet, Dislocation and Kink Dynamics in Fcc Metals Studied by Mechanical Spectroscopy, Materials Science and Engineering a-Structural Materials Properties Microstructure and Processing 164, 42 (1993).

[88] S. A. Golovin, Methods of mechanical spectroscopy in physical metallurgy, Metal Science and Heat Treatment 44, 227 (2002). 
[89] E. Bonetti, and L. Pasquini, Mechanical spectroscopy of nanocrystalline metals: Structure and anelastic behavior, Journal of Electronic Materials 28, 1055 (1999).

[90] D. O. Miles, G. C. Knollman, A. S. Hamamoto, and G. C. Norstrom, Mechanical Shear Relaxation Spectroscopy in Experimental Viscoelasticity, Journal of Applied Polymer Science 9, 2209 (1965).

[91] E. B. Hermida, and F. Povolo, Distribution Function Parameters Determined from Dynamic-Mechanical Spectroscopy Data, Physica Status Solidi B-Basic Research 182, 301 (1994).

[92] R. N. Kleiman et al., Single-Crystal Silicon High-Q Torsional Oscillators, Review of Scientific Instruments 56, 2088 (1985).

[93] R. N. Kleiman, G. Agnolet, and D. J. Bishop, 2-Level Systems Observed in the Mechanical-Properties of Single-Crystal Silicon at Low-Temperatures, Physical Review Letters 59, 2079 (1987).

[94] P. Rösner, K. Samwer, R. O. Pohl, and S. Schneider, Use of a double-paddle oscillator for the study of metallic films at high temperatures, Review of Scientific Instruments 74, 3395 (2003).

[95] X. Liu et al., On the modes and loss mechanisms of a high $Q$ mechanical oscillator, Applied Physics Letters 78, 1346 (2001).

[96] S. Timoshenko, and J. N. Goodier, Theory of elasticity (McGraw-Hill, New York, 1987).

[97] P. Rösner, Dissertation, Mechanische Relaxation in komplexen Fluiden (GeorgAugust-Universität, Göttingen, 2004).

[98] R. Buchdahl, R. J. Morgan, and L. E. Nielsen, Free Vibrating Reed Apparatus for Measurement of Internal Friction and Youngs Modulus down to 4k, Review of Scientific Instruments 41, 1342 (1970).

[99] T. Scharf, and H. U. Krebs, In situ mechanical spectroscopy of laser deposited films using plasma plume excited reed, Review of Scientific Instruments 77 (2006).

[100] H. Parkus, Mechanik der festen Körper (Springer, Wien [u.a.], 1995).

[101] P. E. Dyer, Excimer laser polymer ablation: twenty years on, Applied Physics aMaterials Science \& Processing 77, 167 (2003).

[102] E. Süske et al., Mechanism of poly(methyl methacrylate) film formation by pulsed laser deposition, Journal of Applied Physics 100 (2006).

[103] H. U. Krebs, and O. Bremert, Pulsed-Laser Deposition of Thin Metallic Alloys, Applied Physics Letters 62, 2341 (1993).

[104] H. U. Krebs et al., Structure of laser-deposited metallic alloys and multilayers, Thin Solid Films 275, 18 (1996).

[105] S. Fähler, M. Störmer, and H. U. Krebs, Origin and avoidance of droplets during laser ablation of metals, Applied Surface Science 110, 433 (1997).

[106] A. Gibaud, and S. Hazra, X-ray reflectivity and diffuse scattering, Current Science 78, 1467 (2000).

[107] M. Wind et al., Structure of amorphous poly-(ethylmethacrylate): A wide-angle x-ray scattering study, Journal of Chemical Physics 122 (2005). 
[108] J. Dechant, Ultrarotspektroskopische Untersuchungen an Polymeren (AkademieVerlag, Berlin, 1972).

[109] S. Lazare, J. Lopez, and F. Weisbuch, High-aspect-ratio microdrilling in polymeric materials with intense KrF laser radiation, Applied Physics a-Materials Science \& Processing 69, S1 (1999).

[110] L. E. Manring, Thermal-Degradation of Poly(Methyl Methacrylate) .4. Random SideGroup Scission, Macromolecules 24, 3304 (1991).

[111] J. F. Rabek, Photodegradation of polymers : physical characteristics and applications ; with 26 tables (Springer, 1996).

[112] C. E. Mortimer, and U. Müller, Chemie: das Basiswissen der Chemie (Thieme, Stuttgart [u.a.], 2001).

[113] B. Lösekrug, A. Meschede, and H. U. Krebs, Pulsed laser deposition of smooth poly (methyl methacrylate) films at 248 nm, Applied Surface Science 254, 1312 (2007).

[114] G. B. Blanchet, P. Cotts, and C. R. Fincher, Incubation: Subthreshold ablation of poly(methyl methacrylate) and the nature of the decomposition pathways, Journal of Applied Physics 88, 2975 (2000).

[115] H. H. Jellinek, and M. D. Luh, Thermal Degradation of Polymethylmethacrylate Energies of Activation, Makromolekulare Chemie-Macromolecular Chemistry and Physics 115, 89 (1968).

[116] B. Isik-Yürüksoy, S. Senel, and O. Güven, Thermal degradation of poly(ethyl methacrylate) and its copolymer with poly(ethyl acrylate), Journal of Thermal Analysis 48, 783 (1997).

[117] H.-G. Elias, Makromoleküle (Hüthig \& Wepf, Basel [u.a.], 1990).

[118] C. Zener, Elasticity and anelasticity of metals (Univ. of Chicago Pr., Chicago, 1965).

[119] B. H. Houston et al., Thermoelastic loss in microscale oscillators, Applied Physics Letters 80, 1300 (2002).

[120] R. Hull, Properties of crystalline silicon (INSPEC, London, 1999).

[121] R. D. Biggar, and J. M. Parpia, Magnetic field effects on boron-doped Si oscillators, Physical Review B 56, 13638 (1997).

[122] C. L. Spiel, R. O. Pohl, and A. T. Zehnder, Normal modes of a Si(100) double-paddle oscillator, Review of Scientific Instruments 72, 1482 (2001).

[123] H. A. Kramers, Die Dispersion und Absorption von Röntgenstrahlen, Phys. Z. 30, 522 (1929).

[124] R. D. L. Kronig, On The Theory of Dispersion of X-Rays, J. Opt. Soc. Am. 12, 547 (1926).

[125] K. S. Cole, and R. H. Cole, Dispersion and Absorption in Dielectrics I. Alternating Current Characteristics, The Journal of Chemical Physics 9, 341 (1941).

[126] S. Strella, and R. Zand, Dielectric Studies of the Methacrylate Series .1. Polyethyl Methacrylate, Journal of Polymer Science 25, 97 (1957).

[127] Y. Ishida, and K. Yamafuji, Studies on Dielectric Behaviors in a Series of PolyalkylMethacrylates, Kolloid-Zeitschrift 178, 97 (1961). 
[128] M. S. Dionisio, J. J. Mouraramos, and G. Williams, Dielectric-Relaxation in Poly(nAlkyl Methacrylate)s and Their Mixtures with P-Nitroaniline, Polymer 35, 1705 (1994).

[129] R. Rotzoll, Massenfeinbestimmung am lösbaren Anteil laserdeponierter PEMA-Filme., (2009), Persönliche Mitteilung.

[130] G. B. Mckenna, Glassy State Relaxation and Aging, Abstracts of Papers of the American Chemical Society 208, 3 (1994).

[131] A. Chapiro, Radiation chemistry of polymeric systems (Interscience Publishers, New York, 1962).

[132] B. Fuchs, Härteuntersuchungen an glatten, laserdeponierten PMMA-Schichten., (2009), Persönliche Mitteilung.

[133] B. Fuchs et al., Hardening of smooth pulsed laser deposited PMMA films by heating Applied Physics a-Materials Science \& Processing 98, 711 (2010).

[134] M. Beiner, Relaxation in poly(alkyl methacrylate)s: Crossover region and nanophase separation, Macromolecular Rapid Communications 22, 869 (2001).

[135] M. Cizmecioglu, R. F. Fedors, S. D. Hong, and J. Moacanin, Effect of Physical Aging on Stress-Relaxation of Poly(Methyl Methacrylate), Polymer Engineering and Science 21, 940 (1981).

[136] J. D. Ferry, Viscoelastic properties of polymers (Wiley, New York, 1980).

[137] A. Meschede, T. Scharf, H. U. Krebs, and K. Samwer, Mechanical spectroscopy of laser deposited polymers, Applied Physics a-Materials Science \& Processing 93, 599 (2008).

[138] S. C. Kuebler, A. Heuer, and H. W. Spiess, Geometry of main chain motions in poly(ethyl methacrylate) monitored by 3D difference correlated NMR, Macromolecules 29, 7089 (1996).

[139] F. Garwe et al., Influence of cooperative alpha dynamics on local beta relaxation during the development of the dynamic glass transition in poly(n-alkyl methacrylate)s, Macromolecules 29, 247 (1996).

[140] S. Kahle et al., Glass-transition cooperativity onset in a series of random copolymers poly(n-butyl methacrylate-stat-styrene), Macromolecules 30, 7214 (1997).

[141] S. H. Zhang, X. Jin, P. C. Painter, and J. Runt, Broad-band dielectric study on poly(4vinylphenol)/poly(ethyl methacrylate) blends, Macromolecules 35, 3636 (2002).

[142] K. Schröter et al., Dielectric spectroscopy in the alpha beta splitting region of glass transition in poly(ethyl methacrylate) and poly(n-butyl methacrylate): Different evaluation methods and experimental conditions, Macromolecules 31, 8966 (1998).

[143] K. L. Ngai, T. R. Gopalakrishnan, and M. Beiner, Relaxation in poly (alkyl methacrylate)s: Change of intermolecular coupling with molecular structure, tacticity, molecular weight, copolymerization, crosslinking, and nanoconfinement, Polymer 47, 7222 (2006).

[144] P. Rösner et al., Comparison of mechanical and dielectric relaxation processes in laser-deposited poly(methyl methacrylate) films, New Journal of Physics 8 (2006).

[145] D. S. Fryer, P. F. Nealey, and J. J. de Pablo, Thermal probe measurements of the glass transition temperature for ultrathin polymer films as a function of thickness, Macromolecules 33, 6439 (2000).

[146] R. Seemann et al., Dynamics and structure formation in thin polymer melt films, Journal of Physics-Condensed Matter 17, S267 (2005). 


\section{Danksagung}

Mein besonderer Dank gilt an dieser Stelle Herrn Prof. Dr. Uli Krebs für die einmalige Möglichkeit, in seiner Arbeitsgruppe promovieren zu können. Sein stets offenes Ohr für Fragen und Anliegen, verbunden mit den zugehörigen Antworten und Anstößen haben wesentlich zum Gelingen dieser Arbeit beigetragen. Ohne ihn und das ausgezeichnete Arbeitsklima innerhalb der Gruppe hätte ich nicht nur niemals Licht am Ende der Doktorarbeit gesehen, auch der Weg dorthin wäre ziemlich dunkel gewesen.

In gleichen Atemzug geht der Dank ungedämpft weiter an die weiteren Mitglieder der Arbeitsgruppe: Begonnen mit den Ehemaligen PMMA-lern Erik Süske und Thorsten Scharf, die mit ihren unermüdlichen Untersuchungen die wissenschaftlichen Grundlagen dieser Arbeit gelegt haben, geht der Dank weiter an Sebastian Dreyer und Jörg Faupel für viele Erkenntnisse nicht nur der physikalischen Art. Den aktuellen Kollegen der PLD-Gruppe, sortiert nach Dienstalter Johanna Röder, Britta Fuchs, Tobias Liese, Susanne Seyffarth, Felix Schlenkrich, Sarah Hoffmann und Benedikt Ernst danke ich für die freundschaftliche, kompetente, kreative und meist äußerst inspirierende Atmosphäre rund um den zweiten Stock. Dahin gehören natürlich auch Conni Mewes und Michael Malchow, bei denen die detaillierte Dankesliste sicherlich zu lang wäre - daher nur kurz: Danke!

Besonderer fachlicher Dank gilt noch Jörg Hoffmann, der einen Großteil zur Interpretation der dielektrischen Untersuchungen beigetragen hat. Auch dem Rest der Sputtertruppe sei hier gedankt für die ausgezeichnete Zusammenarbeit und die freundschaftliche Atmosphäre.

Für die kompromisslose Aufnahme in seine Glasgruppe, die Teilung seiner unerschöpflichen Expertise auf dem Gebiet der Glasphysik, viele wertvolle Ratschläge, stete Unterstützung und nicht zuletzt für die Übernahme des Korreferats danke ich herzlich Herrn Prof. Dr. Konrad Samwer. Besonderer Dank für intensiven Austausch, viele Diskussionen und ungeordnete Zusammenarbeit gilt auch den Mitgliedern der Glasgruppe, stellvertretend hier durch Dennis Bedorf repräsentiert, der sich den Dank bei gemeinsamen Kämpfen am Paddel mehr als verdient hat.

Auch möchte ich mich bei allen Mitarbeitern des Instituts für Materialphysik bedanken für die hervorragende Arbeitsatmosphäre. Insbesondere den technischen Mitarbeitern danke ich herzlich für ihre freundliche und zuverlässige Unterstützung. 
Allen Mitgliedern des Sonderforschungsbereichs 602 sowie des Graduiertenkollegs 782, insbesondere Herrn Prof. Dr. Martin Suhm und Herrn Prof. Dr. Philipp Vana vom Institut für Physikalische Chemie, danke ich für die Bereitstellung eines vorbildlichen wissenschaftlichen Umfelds, das seines Gleichen sucht.

Ich bedanke mich bei allen meinen Freunden, die mich außerhalb meiner Zeit im Institut durch alle Höhen und Tiefen der letzten Jahre begleitet haben.

Ein besonderes Dankeschön geht an meine Freundin Anke für Ihre moralische Unterstützung, den Kampf gegen meinen Schweinehund, die Geduld, das Last-Minute-Korrekturlesen und überhaupt. Danke. Anke.

Ganz besonders möchte ich mich bei meinen Eltern und meiner Familie bedanken, ohne die mein Studium und sicherlich auch diese Arbeit niemals möglich gewesen wäre. 




\section{Lebenslauf}

\section{Persönliche Daten}

Name: Andreas Meschede

Geburtsdaten: 09. März 1977 in Warstein

Staatsangehörigkeit: deutsch

\section{Schulbildung}

1983 - 1987: Kath. Grundschule der Stadt Warstein

1987 - 1996: Gymnasium der Stadt Warstein

11. Juni 1996: Allgemeine Hochschulreife

\section{Studium}

1997 - 2000: Grundstudium der Physik an der Georg-August-Universität Göttingen

04. Juli 2000: Diplomvorprüfung Physik

2000 - 2005: Hauptstudium der Physik an der Georg-August-Universität Göttingen Diplomarbeit in der Arbeitsgruppe von Prof. Dr. H.-U. Krebs am Institut für Materialphysik

20. Januar 2005: Diplomhauptprüfung Physik Titel der Arbeit: Wachstum und Eigenschaften laserdeponierter $\mathrm{MgO} / \mathrm{Fe}$ und $\mathrm{Ti}-\mathrm{Sc} / \mathrm{Fe}$-Cr-Schichtsysteme

\section{Promotion}

seit 01. Februar 2005: Doktorand in der Arbeitsgruppe von Prof. Dr. H.-U. Krebs am Institut für Materialphysik

Wissenschaftlicher Mitarbeiter des SFB 602 - TP B8 

